\title{
Non-human primate iPS cells for cell replacement therapies and human cardiovascular disease modeling
}

\author{
Dissertation \\ for the award of the degree \\ "Doctor rerum naturalium" (Dr.rer.nat.) \\ of the Georg-August-Universität Göttingen
}

within the doctoral program Biology of the Georg-August

University School of Science (GAUSS)

submitted by

Ignacio Rodríguez Polo

from Madrid, Spain

Göttingen, 2019 


\section{Thesis Committee}

Prof. Dr. Rüdiger Behr, Platform Degenerative Diseases, German Primate Center (Deutsches Primatenzentrum, DPZ), Göttingen.

Prof. Dr. med. Wolfram-Hubertus Zimmermann, Institute of Pharmacology and Toxicology, University Medical Center, Göttingen.

Prof. Dr. Gregor Bucher, Departament Evolutionary Developmental Genetics, GeorgAugust-Universität Göttingen, GZMB, Göttingen.

\section{Members of the Examination Board}

Prof. Dr. Rüdiger Behr, Platform Degenerative Diseases, German Primate Center (Deutsches Primatenzentrum, DPZ), Göttingen.

Prof. Dr. med. Wolfram-Hubertus Zimmermann, Institute of Pharmacology and Toxicology, University Medical Center (UMG), Göttingen.

Prof. Dr. Gregor Bucher, Departament Evolutionary Developmental Genetics, GeorgAugust-Universität Göttingen, GZMB, Göttingen.

\section{Further members of the Examination Board}

Dr. Ufuk Günesdogan, Dept. Developmental Biology (GZMB), Georg-AugustUniversität, Göttingen.

Prof. Dr. Susann Boretius, Functional Imaging Unit, German Primate Center (Deutsches Primatenzentrum, DPZ), Göttingen.

Prof. Dr. med. Ralf Dressel, Institute of Cellular and Molecular Immunology, University Medical Center (UMG), Göttingen.

Date of the oral examination: October 29th, 2019. 

"The love of complexity without reductionism makes art; the love of complexity with reductionism makes science." Edward O. Wilson 


\section{Declaration}

I hereby declare that this thesis has been written independently, without use of other sources and aids than those cited.

Ignacio Rodríguez Polo

Göttingen, September $18^{\text {th }} 2019$ 


\section{Table of Contents}

I. Table of Contents .................................................................................................6

II. Abstract ................................................................................................................8

III. Introduction .....................................................................................................................10

1 Stem cells.........................................................................................................................11

1.1 Induced pluripotent stem cells (iPSC) and reprogramming .............................................12

1.2 Induced pluripotent stem cells characterization ........................................................................ 14

$1.3 \quad$ Non-human primate-iPSC (NHP-iPSC).................................................................................. 15

2 Application of pluripotent stem cells in cardiovascular research ................17

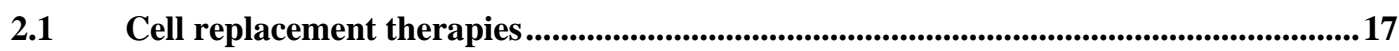

2.2 Drug discovery and toxicity test ..............................................................................18

$2.3 \quad$ Disease modeling ........................................................................................................18

3 Non-human primates as animal models in cardiovascular research.............19

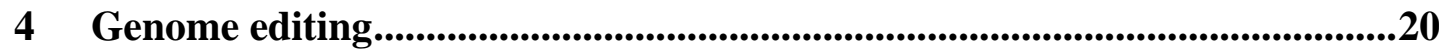

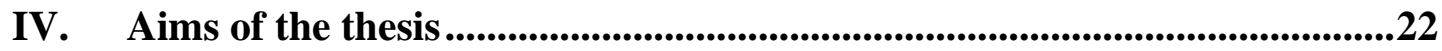

V. Chapter 1: Baboon induced pluripotent stem cell generation by piggyBac

transposition of reprogramming factors .............................................................24

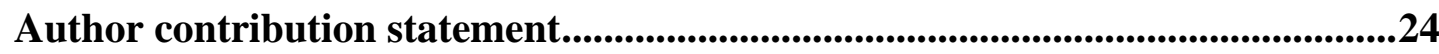

VI. Chapter 2: Reproducible Primate iPSC Generation, Cultivation, and Cardiac Differentiation under Chemically Defined Conditions .............................39

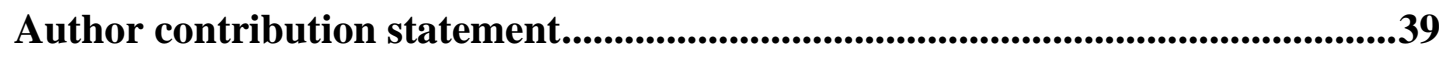

VII. Chapter 3: A piggyBac-based platform for genome editing and clonal rhesus macaque iPSC line derivation .................................................................74

Author contribution statement.........................................................................74

VIII. Chapter 4: An iPSC-based preselection platform for disease-inducing genetic modifications in non-human primates 
Author contribution statement 108

IX. Discussion ....................................................................................................................145

1 Refinement of NHP-iPSC generation and culture .......................................145

$1.1 \quad$ Characterization of NHP-iPSCs ...........................................................................................147

2 Efficient generation of functional NHP-iPSC cardiomyocytes....................149

3 Modeling cardiovascular disease in NHP-iPSCs.........................................150

4 Exploring new therapies for monogenic cardiovascular diseases ...............152

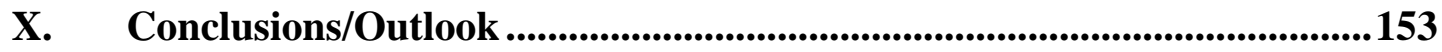

XI. References Introduction and Discussion .............................................155

XII. Acknowledgments..........................................................................167

XIII. Curriculum Vitae..........................................................................168 


\section{Abstract}

Induced pluripotent stem cells (iPSC) have revolutionized biomedical research due to their versatility. They have potential for regenerative medicine, drug testing, disease modeling and developmental biology. The development of cell-based regenerative therapeutics and the subsequent treatment of human degenerative diseases require testing in predictive animal models like non-human primates (NHP). Therefore NHPiPSC in conjunction with NHP are highly important to conduct preclinical studies. I have generated and established in the context of this collaborative effort, to our knowledge for the first time, transgene- and feeder-free iPSCs from three NHP species, namely the rhesus macaque (Macaca mulatta), the olive baboon (Papio anubis) and the common marmoset (Callithrix jacchus), as well as human iPSC. For the generation of the different NHP-iPSC lines, we explored different reprogramming approaches and culture conditions. Eventually, we succeeded in establishing a universal reprogramming protocol for primate fibroblasts. Furthermore, we successfully adapted a differentiation protocol for human iPSC into functional cardiomyocytes (iPSC-CM) to NHP-iPSC.

The generated NHP-iPSC, besides their potential to test stem cell-based regeneration therapies, can also be used for disease modeling. The generation of genetically modified NHP animal models for human diseases may contribute to drug testing and enhances pathophysiological studies during disease onset and progression in a controlled experimental setup. However, in order to safely introduce predefined mutations into the (embryonic) genome of monkeys, the efficiency and accuracy of a genome editing approach needs to be pre-screened, ideally using iPSC of the respective NHP species. Due to the similarities between iPSC and pluripotent cells from the early embryo, it is possible and suggested to study the efficiency, efficacy and accuracy of the editing approach using iPSC. Furthermore, the capability of the iPSC to differentiate into different somatic cell types may allow the prediction of aspects of the in vivo phenotype of genetically modified NHP. In summary, I have generated and established in the context of these collaborative studies a set of NHPiPSC-lines that (1) can be used for preclinical testing of cell replacement therapies and (2) forms the basis of an in vitro platform that allows the validation of genome editing approaches, e.g by CRISPR/Cas, for the generation of genetically modified NHP. With this approach, we established an in vitro testing and preselection platform for genetic modifications before their application in vivo in NHP. Besides its scientific value, this platform will also contribute to the $3 \mathrm{Rs}$ (reduce, replace, refine) in animal experimentation, which are of particular importance in the context of NHP research.

The current dissertation has been written following the pseudo-cumulative structure registered in the Official Bulletin I no. 28 dated 22.06.2018, doctoral degree regulations (RerNat-O) GAUSS. This thesis includes four different chapters / manuscripts (both published and not published), each independent and completely understandable as a single manuscript. As all chapters reflect the evolution of 
techniques and results during this Ph.D., they are grouped under a general introduction and discussion, giving a full overview and analysis of the thesis. 


\section{Introduction}

Cardiovascular diseases (CVDs) are one of the major causes of death worldwide. This group of pathologies affects human cardiac and vascular systems and usually presents a complex etiology ${ }^{1}$. CVDs cover a broad range of diseases, like ischemic heart disease, acute myocardial infarction, cardiomyopathies, atherosclerosis, or congenital heart disease ${ }^{2}$. Due to the high incidence of these pathologies in society, it is crucial to investigate their cause, and progression to develop efficient treatments ${ }^{1}$. For a better understanding of these diseases, it is necessary to strengthen basic research and epidemiological studies. Complementarily, it is of utmost importance to establish bridges between the above mentioned disciplines and clinical applications using animal models that share the complexity of human cardiovascular (CV) system. Most of experimental $\mathrm{CV}$ in vivo research relies on rodent models. Rodents significantly contribute to the progress of our understanding of CVDs ${ }^{3}$. However, due to major differences between rodent and human $\mathrm{CV}$ system (heartbeat or innate resistance to CVD) they fail to address a variety of research questions. Therefore it is necessary to complement rodent studies with large animal models that will allow a better translation of the results obtained to the human pathological set ${ }^{2}$.
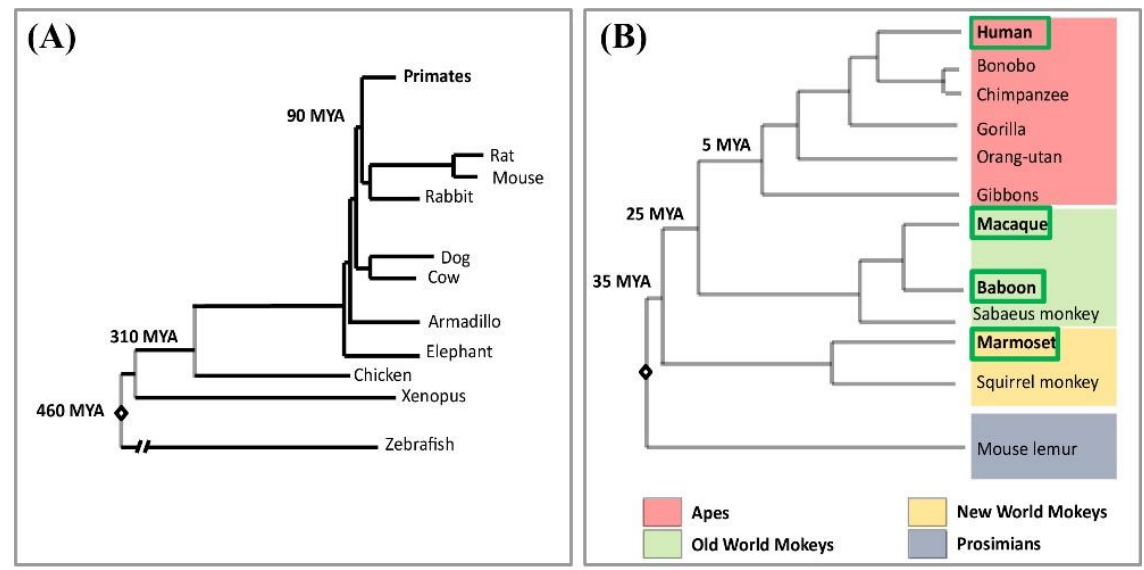

(C)

(C')
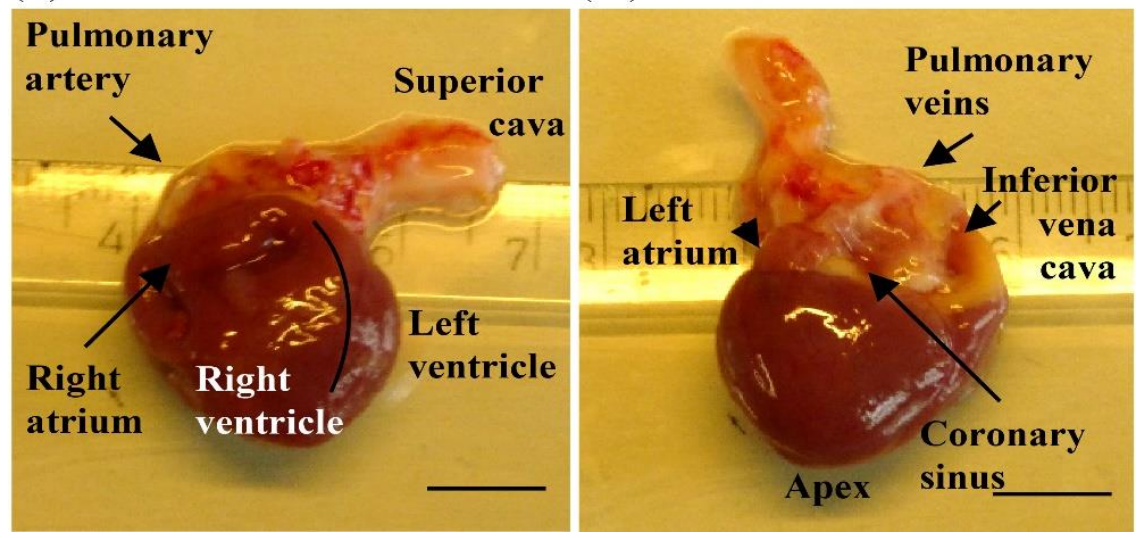

Figure 1. Phylogenetic comparison of human and NHP and anatomy of the NHP heart. (A) Phylogenetic tree of selected species (Adapted from ${ }^{4}$ and ${ }^{5}$ ). (B) Phylogenetic tree of primates (Adapted from ${ }^{6}$ ). Evolutionary distance is shown in "millions of years ago" (MYA). (C, $\mathbf{C}^{\prime}$ ) Anatomical analysis of an adult female marmoset heart. (C) Frontal view. (C') Posterior view. Scale bar $1 \mathrm{~cm}$. 
The main translational animal models in CV research are pig, dog, sheep, and nonhuman primates (NHP) ${ }^{7} 89$. NHP as our closest relatives, share a primate-specific genomic constitution and high similarities in anatomy, physiology, and behavior (Fig. 1) (Table 1) ${ }^{10}$. Therefore, NHP are well-recognized models and have a long history in biomedical research. Besides the traditional usage in toxicology, their recognition is increasing for other applications. Although, NHP account for only 5\% of all researchrelated animal models, they are now increasingly used in disease modeling and testing of advanced therapy medical products (ATMP) 27111213 .

However, in addition to animal models, it is necessary to consider in vitro state of the art tools to expand the frontiers of this research field. Stem cells, due to their versatility, represent a very interesting approach in this context. Within the broad range of stem cell applications, they play a vital role in CV research. Patient-specific induced pluripotent stem cells (iPSC) have been essential to study disease development and progression. Furthermore, iPSC present an interesting approach towards regenerative therapies for the CV system.

\begin{tabular}{|c|c|c|c|}
\hline & \multicolumn{2}{|c|}{ Old World Monkeys } & \multirow{2}{*}{$\begin{array}{c}\begin{array}{c}\text { New World } \\
\text { Monkeys }\end{array} \\
\text { Callithrix jacchus }\end{array}$} \\
\hline & Papio hamadryas & Macaca mulatta & \\
\hline Common name & Hamadryas baboon & Rhesus monkey & Marmoset \\
\hline Longevity (Max) (y)* & $37.5^{141516}$ & $40^{17}$ & $16.5-22.8^{1418}$ \\
\hline Average life span (y) & $30^{19}$ & $27^{17}$ & $5-7.07^{18} 20$ \\
\hline Body temperature $\left({ }^{\circ} \mathrm{C}\right)$ & $38^{1516}$ & $37.7^{21}$ & $37-39^{22}$ \\
\hline Gestation (d) & $171-179^{23}$ & $165^{24}$ & $143-144^{20}$ \\
\hline Male sexual maturity (d) & $1762^{1516}$ & $1095-1277^{25}$ & $382^{15}$ \\
\hline Female sexual maturity (d) & $1514^{1516}$ & $912-1095^{25}$ & $477-547^{18} 20$ \\
\hline Litter size & $1^{1516}$ & $1^{15}$ & $2(3)^{1820}$ \\
\hline Litters per year & $0.8^{1516}$ & $1^{15}$ & $2(3) 1820$ \\
\hline Weight at birth (g) & $814^{1516}$ & $464^{15}$ & $26.5^{20}$ \\
\hline Adult weight (g) & $\begin{array}{c}20000(\mathrm{f})- \\
38000(\mathrm{~m})^{26}\end{array}$ & $9700^{27}$ & $<400^{18}$ \\
\hline
\end{tabular}

Table 1: Phylogenetic proximity of non-human primates and human is reflected in highly similar physiological and anatomical features. Represented in the table are selected biological key parameters of the three animal models considered in this study: Baboon (Papio anubis is the species used in this project, data shown for Papio hamadryas), rhesus macaque (Macaca mulatta), and common marmoset (Callithrix jacchus). (d) days, (g) grams, (y) years, $\left({ }^{\circ} \mathrm{C}\right)$ degrees Celsius, (f) female, and (m) male.

\section{Stem cells}

Stem cells are characterized by their capability of self-renew and differentiation into at least one cell type ${ }^{28}$. Stem cells perform essential functions during development and in tissue homeostasis. They can be categorized into three groups according to their developmental potency: pluripotent, multipotent, and unipotent stem cells. All adult stem cells are either multipotent or unipotent. Multi- and unipotent cells demonstrate limited differentiation capacities into one or few cell types from the same embryonic lineage. In contrast, pluripotent stem cells (PSC) can differentiate into all cells of the organism. As mentioned, pluripotency can be defined as the regulative capability of a 
single cell to give rise to cells of all germ layers ${ }^{29}$. Embryonic pluripotent cells are present in the epiblast, of the post-implantation pre-gastrulation embryo (blastocyst stage) ${ }^{30}$. During embryo development, these cells give rise to all cell types of the organism but they are not able to generate the extra-embryonic tissues ${ }^{28} 30$.

Embryonic pluripotent cells can be isolated from a developing embryo and, mimicking their natural niche, cultured in vitro as embryonic stem cells (ESC). ESC lines have been established from human and many different animal models, including mouse, rat, macaque, and baboon 31323334 . The possibility of maintaining and expanding these cells in vitro plus differentiating them into specific cell types raised new possibilities concerning novel treatments and disease modeling. Using PSCs, it is possible to generate tissue-specific cells, like cardiomyocytes, and utilize them for human disease modeling. Besides the promise of applying ESC in the clinical scenario, there are ethical concerns associated with their usage considering the sacrifice of an embryo for accessing the cells ${ }^{35}$.

In 2006 Takahashi and Yamanaka discovered a method to awake endogenous pluripotency in terminally differentiated cells. This was achieved by ectopic expression of $O C T 4$, SOX2, KLF4, and $c M Y C$, genes designated as Yamanaka factors. The technique was first published using mouse and afterward using human fibroblasts 3637 . The generated pluripotent stem cells were termed induced pluripotent stem cells (iPSC). iPSC share many characteristics with ESC, including gene expression profiles, DNA methylation, and covalent histone modifications ${ }^{38}$. Furthermore, they circumvent the ethical controversy associated with the destruction of embryos. The strong similarities between both types of PSC allowed the translation of the in vitro culture conditions from ESC to iPSC 38 39.

\subsection{Induced pluripotent stem cells (iPSC) and reprogramming}

Reprogramming can be defined as the process of remodeling the epigenetic landscape of a cell. The first successful attempts of reprogramming took place much earlier than the discovery of iPSCs by Takahashi and Yamanaka. Briggs and J. King first reported successful reprogramming in 1950. Here, somatic nuclear transfer from an advance blastula into an enucleated northern leopard frog (Rana pipiens) egg, resulted in the reprogramming of the oocyte ${ }^{40}{ }^{41}$. The Nobel Prize-awarded Sir John Gurdon used tadpole nuclei for successful cloning of the frog Xenopus laevis using the same technique ${ }^{39}$. These and subsequent studies grounded the basics of reprogramming. However, until the discovery of iPSCs, it was still necessary to use embryonic tissue.

iPSCs can differ from each other depending on the cell type from which they were derived and the reprogramming approach used ${ }^{38}$. It has been shown that almost any cell in the human body can be reprogrammed (e.g., skin fibroblast, stomach cells, liver cells, or keratinocytes) 42434445 . As mentioned above, the process of reprogramming begins with the exogenous expression of the Yamanaka factors. Later approaches used further factors, such as $N A N O G$ and $L I N 28$, for "reprogramming resistant" cells or species.

OCT4 (POU5F1), SOX2, and KLF4 transcription factors work synergistically towards pluripotency by inducing dynamic changes in gene expression (Fig. 2) ${ }^{46} 47$. These three transcription factors co-occupy the promoters of many reprogramming-related 
genes, awaking endogenous pluripotency in the somatic cells ${ }^{48}$. OCT4 acts alone or forms a heterodimer with SOX2. Both SOX2 and OCT4 can differentially bind the DNA depending on the configurations of their protein structure. OCT4 is related to the maintenance of the self-renewal capabilities in PSC ${ }^{46}{ }^{48}$, while SOX2 facilitates the establishment of the trophectoderm lineage ${ }^{49}$. Additionally, MYC overexpression represses lineage-specifying transcription factors and stimulates cell cycle via promotion of DNA synthesis 5051 . Reprogramming without MYC has been demonstrated for mouse, however resulting in low efficiencies ${ }^{51}$. The initial experiments by Takashi and Yamanaka used c-MYC; although, this factor is constitutively expressed in cancer cells. Therefore refined reprogramming approaches substitute c-MYC for other members of the MYC family, e.g., 1-MYC ${ }^{52}$.

LIN28 is occasionally included to assist reprogramming in combination with the canonical four factors. This RNA-binding protein support pluripotency via regulation of the microRNAs let-7 family, which promote differentiation ${ }^{53}{ }^{54}$. Furthermore, the transcription factor NANOG is also often used to complement the standard reprogramming cocktail. OCT4, NANOG, and SOX2 form an interconnected autoregulatory loop binding each other's endogenous promoters and contributing to the pluripotency stabilization of the iPSC (Fig. 2) ${ }^{48}$.

A

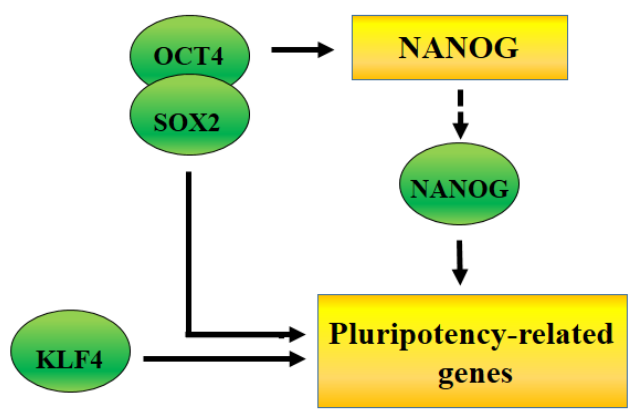

B

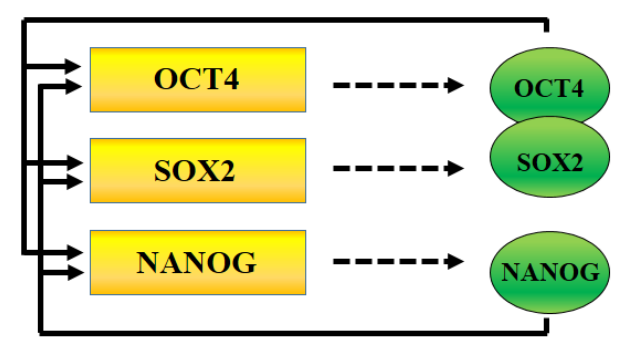

Figure 2: Transcription factor-induced reprogramming of somatic cells into iPSCs. (A) Represented regulatory circuit of pluripotency in ESC and iPSCs detailing the transcription factors KLF4, OCT4, SOX2, and NANOG. (B) Autoregulatory positive loop between OCT4, SOX2, and NANOG. Green circles indicate transcriptional activators and rectangles gene promoters. Binding of regulators (green ovals) to promoters (yellow boxes) indicated by a solid arrow and gene expression by dashed arrows. Adapted from ${ }^{48}$.

There are multiple strategies to induce overexpression of the Yamanaka factors in somatic cells. Different reprogramming approaches can be characterized according to the delivery method of the factors. To the present, delivery of DNA, RNA, and protein have been tested. DNA based delivery is the most utilized amongst reprogramming methods, although these methods entail the risk of altering the endogenous genomic DNA (gDNA). RNA or protein-based methods are considered to be safer; however, the reprogramming efficiencies are lower 5556 .

Within DNA based approaches, two types can be distinguished: integrative and nonintegrative approaches. Integrative methods insert the reprogramming construct into the gDNA. This integration can be either reversible or irreversible. Traditionally, iPSC have been generated using integrative methods, using, e.g., retro- and lentiviruses. These systems demonstrate high efficiencies; however, the genome of the cells is 
permanently modified. The constitutive expression of the reprogramming factors can lead to mutagenesis or failing differentiation. Hence, they make the cells inappropriate for transplantation studies. On the contrary, the piggyBac system represents an integrative, yet reversible reprogramming approach. It consists of a transposon and a transposase, in which expression of the transposase will lead to the excision of the transposable cassette from the parental vector. The cassette will then integrate into a chromosomal TT/AA site ${ }^{57}$. One of the main advantages of this system is that it can be excised, leaving no footprint in the genome. The piggyBac system has been used to efficiently reprogram human, mouse, marmoset and baboon fibroblasts 57585960 .

Non-integrative DNA-based methods offer the possibility to generate fully footprintfree iPSC ${ }^{41}$. The cells can be generated using non-integrating viruses, like Adenovirus, or Sendai Virus. Other non-integrative non-viral based reprogramming methods include minicircles or episomal plasmids ${ }^{57}$. The episomal plasmid system allows transient expression of the reprogramming factors without integration. OriP/EBNAbased plasmids allow a temporary but prolonged-expression during few passages. This allows stable reprogramming and increases efficiency. Episomal vector systems have been used to reprogram, e.g., human, macaque, and marmoset cells 425261 .

The safest reprogramming approaches are based on RNAs encoding the reprogramming factors since RNAs cannot integrate into gDNA. Although these approaches have been successfully used, they are either very expensive of have rather a low efficiency 6263 .

\subsection{Induced pluripotent stem cells characterization}

All molecular changes induced during reprogramming are reflected by a drastic epigenetic remodeling, leading to a new phenotype of the cells. Morphology and proliferation changes, giving rise to small highly proliferative cells with a high nucleus/cytoplasm ratio. Even though morphological changes are a crucial aspect to consider, an in-deep characterization of the iPSCs is necessary in order to confirm their pluripotent status ${ }^{42}$. The scientific community agreed to several assays that allow confirmation of pluripotency of the iPSCs ${ }^{42}{ }^{64}$. First, it is important to analyze the changes in expression profiles in comparison to differentiated cells. This allows confirmation of the reactivation of pluripotency markers. Transcript and protein analyses are usually performed for key pluripotency factors like OCT4A, LIN28, and NANOG, among others.

After verification of the reactivation of the pluripotency machinery, the differentiation capabilities are assessed both in vitro and in vivo. The gold standard assay for potency evaluation of rodent iPSC is the chimera assay. Chimeras are generated by injecting iPSCs into a host preimplantation embryo, which is then retransferred into a foster mother. The potency of the iPSCs is evaluated in the organism developed from the chimeric embryo. The contribution of the injected cells in different tissues is subsequently assessed ${ }^{65}$. This method evaluates potency in a highly accurate way; however, due to ethical and practical limitations it is not feasible for human cells or cells from other species.

Further assessments of pluripotency include directed differentiation in vitro and spontaneous differentiation both in vitro and in vivo. Spontaneous differentiation 
describes random differentiation elicited by exposing the iPSCs to a general differentiation environment. In vivo assessment of pluripotency is demonstrated by the teratoma formation assay, in which the test cells are subcutaneously injected into immune-deficient mice. Here, the iPSCs proliferate to form a tumor, which is then analyzed for histological features of the three germ layers ${ }^{66}$. In vitro, cells are introduced to a general differentiation media, resulting in random differentiation. Analysis of the potency is performed by expression analysis of representative markers of the three germ layers. Cells are considered pluripotent if this is found to be true ${ }^{67}$. An alternative option is directed differentiation, in which the iPSCs are differentiated towards a specific cell type, such as neuron- or cardiomyocyte-like cells ${ }^{42}{ }^{68}$. Directed differentiation requires established protocols to imitate the natural differentiation signals during development to generate a specific cell type. Even though this has been achieved for many cells types, for others it remains elusive. Furthermore, the protocols are commonly established for human or mouse iPSCs, often cannot be translated directly to other species.

The establishment of the mentioned assays has contributed to the generation of a robust work-flow for stem cell characterization. However, even though many efforts have been made for standardized reprogramming and pluripotency assessment protocols, there is still high heterogeneity between iPSCs generated in different laboratories. Hence, for the sake of comparability of data from different labs, it is necessary to continue increasing the standards for iPSC generation and assessment. Some efforts in this direction have already been made by the generation of testing systems like Scorecard or PluriTest, which identify specific molecular signatures and functional pluripotency 676970 . Additionally, the standards for clinical-grade human iPSC generation have been defined over the last years. In order to generate high-quality iPSCs and to ensure their comparability between human and other species, it is necessary to define critical quality attributes that all iPSCs need to fulfill ${ }^{42}$. Therefore iPSCs from relevant translational models have to follow human iPSC guidelines ${ }^{64}$.

\subsection{Non-human primate-iPSC (NHP-iPSC)}

NHP models in combination with species-specific iPSC provide the missing link for translation of basic regenerative research in rodents to human clinical trials ${ }^{42}$. NHP present, due to their close phylogenetic relationship, compelling advantages in comparison with other animal models concerning autologous and allogeneic stem cellbased regenerative studies (Fig. 1) ${ }^{42}$. NHP in conjunction with NHP-iPSCs provide a system that allows addressing specific questions going back and forth from in vitro to the in vivo systems. In order to utilize the close phylogenetic proximity of NHP and human and to put gained knowledge onto a solid basis, the generation of a broad panel of NHP-iPSC is necessary ${ }^{42}$.

After the generation of human and mouse iPSC, the first reports of NHP-iPSC followed ${ }^{71} 727374$. In 2008 the first NHP-iPSCs were generated from the macaque ${ }^{75}$. Shortly after, in 2010, two independent publications demonstrated reprogramming of marmoset fibroblast into iPSCs ${ }^{76}$ 77. Since then, a broad range of NHP species have been successfully reprogrammed including baboon, cynomolgus monkey, chimpanzee or drill 74787980 . Although reprogramming protocols have been translated from human to NHP, this process has been shown to be slower and less efficient. For example reprogramming of NHP is still dependent on mouse embryonic feeder cells (MEF) ${ }^{42}$ 
78 81. This limits the preclinical value of the cells since they are mixed with cells from other species and the culture conditions are not standardized.

The difficulties in NHP cell reprogramming and iPSC maintenance illustrate yet unknown characteristics of the regulation of pluripotency in NHP cells, especially considering the high conservation of the main pluripotency related genes across species (Table 2) (Fig. 3). One example is the baboon, from which Navarra and collaborators reported the generation of iPSCs in 2013 and 2018. In both publications, the generated iPSCs needed to be co-cultured with MEFs. These reports underline the difficulty of generating iPSC from this species under conditions homologous to human culture conditions ${ }^{78} 81$. Another example is marmoset reprogramming that has been achieved by different reprogramming methods; however, the maintenance of these cells in feeder-free culture has not been reported yet ${ }^{42}$.

Due to the key role of NHP in translational research, it is necessary to adapt the standards for human iPSC generation to NHP-iPSCs. Human protocols for reprogramming need to be refined towards the molecular particularities of NHP in order to establish them as translational models ${ }^{42}$.

\begin{tabular}{|ccccccc|} 
& Baboon & Macaque & Marmoset & Pig & Rat & Mouse \\
OCT4 & 99,39 & 99,39 & 98,78 & 97,56 & 85,89 & 90,54 \\
KLF4 & 100,00 & 100,00 & 100,00 & 100,00 & 96,55 & 96,55 \\
\hline c-MYC & 95,58 & 98,00 & 97,95 & 97,11 & 95,87 & 96,00 \\
LIN28 & 98,56 & 99,04 & 99,04 & 97,07 & 99,04 & 97,61 \\
\hline NANOG & 93,97 & 97,23 & 96,88 & 85,37 & 76,17 & 77,34 \\
SOX2 & 99,68 & 100,00 & 99,37 & 99,68 & 98,42 & 98,42 \\
\hline ESRRB & 99,54 & 99,54 & 99,54 & 98,85 & 97,92 & 97,46 \\
\hline NR5A2 & 100,00 & 99,76 & 98,78 & 98,51 & 96,16 & 96,16 \\
\hline
\end{tabular}

Table 2. Protein sequence similarity of key pluripotency genes between selected species relevant in the stem cell research field and human. Protein sequence alignments represented as percentage of similarity. 


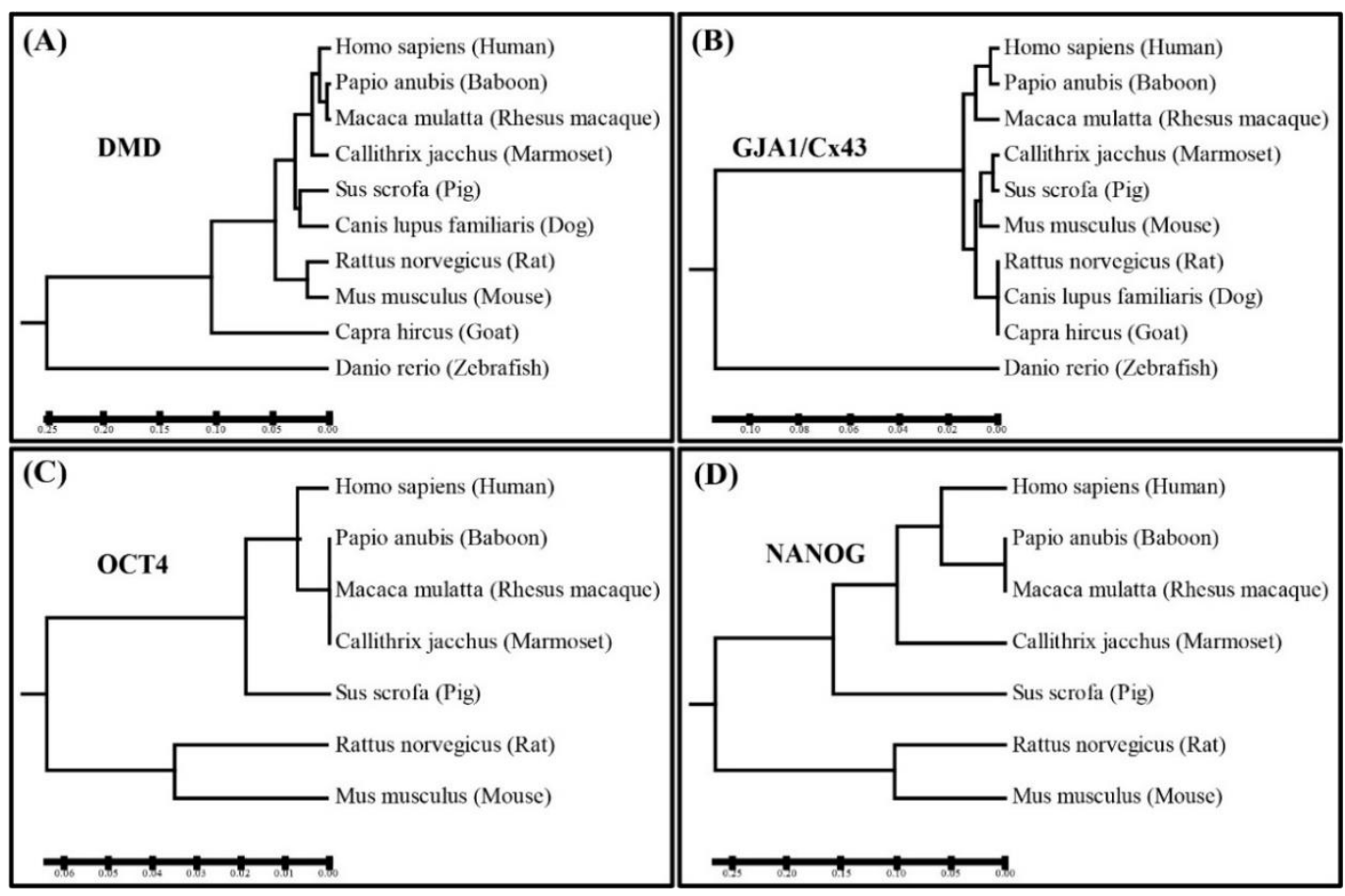

Figure 3. Gene tree showing protein evolution using the UPGMA method, in MEGA 7 software ${ }^{82} 83$. (A)(B) Analysis of the cardiac relevant proteins dystrophin (DMD), and connexin 43 (GJA1/Cx43). Comparison between selected species with relevance in CV research. (C)(D) Analysis of the pluripotency relevant proteins, Octamer binding transcription factor 4 (OCT4/POU5F1), and NANOG. Comparison between selected animal models relevant in stem cell research.

\section{Application of pluripotent stem cells in cardiovascular research}

The versatility of PSC makes them an excellent tool in the context of CV research. PSC can be used to model clinically relevant pathologies in vitro, as a cell source for cell-based regeneration therapies, and drug development and toxicity testing.

\subsection{Cell replacement therapies}

Progressive or sudden loss of functional cells in an organ can be overcome by regenerative cell-based therapies. These approaches open possibilities to treat diseases which to the present are untreatable ${ }^{42}$. PSC present an interesting approach towards cell replacement therapies due to their high proliferation and the differentiation potential.

In the last years, preclinical studies exploring PSC-based therapeutics demonstrated the potential of this pharmacological approach ${ }^{84} 8586$. Notwithstanding the progress made, some open questions still need to be addressed before clinical translation of these therapies. Addressing safety concerns regarding tumorigenicity and immunogenicity are of utmost importance ${ }^{7}$. Furthermore, it is necessary to consider aspects concerning the source of the cells, such as cell type, GMP compliance, or maturation state of the differentiated cells. Finally, it is crucial refining procedures including graft location, delivery protocols, and minimum effective dose 7868788 . 
For addressing these concerns, high-quality iPSCs from relevant animal models are required. This will allow the exploration of the regenerative potential in a controlled research environment. Different preclinical studies using NHP models show promising results using stem cells as a cell source to treat spinal cord injuries ${ }^{89} 90$, retinitis pigmentosa ${ }^{91}$, or myocardial infarction ${ }^{92} 93$ among others 894 .

Myocardial infarction is an ischemic heart disease in which tissue is lost in the heart and substituted by non-contractile scar tissue ${ }^{95}$. Treatment options reversing the damage induced by myocardial infarction do not exist. One potential therapy is to replace or support the scarred tissue by stem cell-based replacement therapies. Application of pluripotent stem cell-derived cardiomyocytes could be one of such therapies ${ }^{95}$. Remuscularization of an infarcted heart has been conducted in different animal models, showing encouraging results. These studies reported the safety of the approach and improvement of the contractile function of the heart after transplantation of the cells 79293 .

\subsection{Drug discovery and toxicity test}

Novel drugs need to be tested before clinical trials to guarantee patient safety. Nowadays, the preclinical testing of medication relies on very few animal models, namely mouse, rat, pig, and NHP. This preclinical phase is crucial, considering that $90 \%$ of the new treatments fail in this phase and do not progress to the clinics ${ }^{2}$. Cardiovascular toxicity, in particular, is one of the major causes of drug withdrawal, accounting for $33 \%$ of drug failure ${ }^{2}$. Cardiac toxicity frequently manifests by altered contractibility or cardiac rhythm, the formation of reactive oxygen species (ROS), apoptosis, and modified cardiac gene expression ${ }^{2}$. Even though the animal models used for drug toxicity testing are well established, iPSCs present an alternative for reproducible and economic drug testing ${ }^{41}$. Additionally, iPSCs may contribute significantly to the reduction of animals used for these purposes.

Although in vivo drug testing will still be required, patient-specific iPSC represent an interesting alternative for preliminary assessment of side effects ${ }^{2}$. Furthermore, patient-specific iPSCs can also contribute to drug screening efforts. One example is the analysis of the contractile function in iPSC-derived CMs exposed to different drugs. The feasibility of iPSC-based toxicity testing is reflected in the development of the first commercially available platforms in the last years (e.g., Ncardia).

\subsection{Disease modeling}

There are two main ways to model genetic human diseases in iPSC: 1- reprogramming patient fibroblasts that contain the disease-inducing genotype into iPSCs, or 2inducing a clinically relevant mutation in wildtype (WT) iPSCs using genome editing tools ${ }^{96}$. Patient-specific iPSCs have the advantage of being a straight forward strategy, allowing the fast generation of a selected disease platform. In contrast, inducing clinically relevant mutation in WT iPSCs is more laborious. However, this approach comes with the advantage of a reduced heterogeneity between replicates and no genetic variation affecting the results. Both systems allow in vitro mimicking of an in vivo phenotype and the possibility of study it under controlled conditions. 
In addition to the in vitro model using human iPSCs with defined mutation, it is important or even necessary to complement these studies with animal models possessing these pathological mutations. Genetically modified NHP will allow researchers to investigate diseases in a model closely related to humans. Speciesspecific iPSC, like NHP-iPSC, could serve as a preliminary approximation to the outcome of these long-term, expensive, and laborious projects. For in vitro disease modeling it is necessary to adapt genome editing tools to the particularities of each species. Nowadays, there are robust protocols to target human and mouse PSC efficiently, but their translatability to PSC from other species is still under investigation ${ }^{97} 98$.

\section{Non-human primates as animal models in cardiovascular research}

To translate basic research into clinical applications, it is necessary to complement rodent studies with preclinical data obtained from large animal models 38999100 . The main large animal models used in translational cardiovascular research are sheep, pig, and NHP 9101 . All mentioned organisms have a higher similarity to human physiology, histology, and genetics in comparison to mouse (Fig. 1, 3) (Table 1, 2 and 3). However, it has to be kept in mind that each one of them presents advantages and disadvantages. Choosing the optimal organism for a study always depends on an in-depth analysis of their characteristics in the context of a specific research question ${ }^{9}$.

NHP can be divided into three big paraphyletic groups, Old World monkeys (OWM), New World monkeys (NWM) and Apes. NWM include five families, namely Callitrichidae, Cebidae, Aotidae, Pitheciidae, and Atelidae, belonging to the parvorder Platyrrhini. OWM are the closest relatives to Apes and constitute the family Cercopithecidae (Fig. 1) ${ }^{4} 5{ }^{5}$. Besides the above-mentioned similarities, OWM additionally have a greater similarity in diet and litter size, in comparison to NWM. These characteristics historically make OWM the preferred models for biomedical research. Nevertheless, NWM are gaining more attention over the last years ${ }^{8}$. The marmoset monkey, for example, shares the primate-specific genomic constitution with OWM while presenting advantages like high reproductive efficiency, low housing cost and easier handling 897 .

NHP, as our closest relatives provide the possibility of studying CVD in animal models with high physiological, genetic, and behavioral similarity to humans ${ }^{102}$. Furthermore, NHP naturally develop diseases homologous human CVDs. Macaques, for example, present diverse age-related pathologies, including various heart diseases, like myocarditis ${ }^{103}$. Additionally, the size of NHP allows utilization of clinical equipment with little to no modifications, e.g., MRI ${ }^{8}$. Another advantage of the size and longer life-span is the possibility of longitudinal studies with several sampling phases. Furthermore, NHP colonies usually follow breeding strategies by which the genetic 
pool is increased. This enriches the populations with genetic variability, which cannot be found in mice ${ }^{8}$.

\begin{tabular}{|c|c|c|c|c|}
\hline Species & $\begin{array}{l}\text { Heart weight } \\
\text { (g) }\end{array}$ & $\begin{array}{l}\text { Heart rate } \\
\text { (bpm) }\end{array}$ & $\begin{array}{c}\text { Systolic } \\
\text { pressure }(\mathrm{mmHg})\end{array}$ & $\begin{array}{l}\text { Coronary artery } \\
\text { diameter (mm) }\end{array}$ \\
\hline Human & $250-480^{8104}$ & $60-100^{105}$ & $60-120^{106}$ & $4.5^{8} 107$ \\
\hline Olive Baboon & $55-116^{108}$ & $60-122105109$ & $113-160^{109}$ & $1.5-2.5^{110}$ \\
\hline Rhesus macaque & $22-30^{111} * / 40^{\#}$ & $165-229^{112}$ & $98-126^{112}$ & $1.40-2.40^{113} * *$ \\
\hline Marmoset & $2.28-2.98^{114}$ & $405-600^{115}$ & $133.5-159^{116}$ & $0.51^{117}$ \\
\hline Pig & $400-500^{104}$ & $65-75^{104106}$ & $135-150^{106}$ & $2,83-3.59^{118}$ \\
\hline Sheep & $240-360^{104}$ & $60-120^{9}$ & $90-115^{9106}$ & $1,65-2.57^{119}$ \\
\hline Dog & $160-420^{104}$ & $70-160^{106}$ & $95-150104106$ & $1.71-1.85^{120}$ \\
\hline Rabbit & $9-11^{104}$ & $\begin{array}{l}120-300^{104} \\
106\end{array}$ & $90-130^{106}$ & $0.99-2^{117121}$ \\
\hline Mouse & $0.14-0.15^{8} \quad 104$ & $310-840^{106}$ & $80-160104106$ & $0.16^{8117}$ \\
\hline
\end{tabular}

Table 3. Selected heart characteristics of human and relevant CVD animal models (adults). Two references were added in the cases where a discrepancy between two publications was found. ${ }^{*}$ ) Heart weight value corresponding to Macaca arctoides. (**) Coronary artery diameter value corresponding to Macaca fascicularis. (\#) Approximately 40 grams is the heart weight values obtained from our macaque colony (German primate center, DPZ, Göttingen). Units used for the different parameters: heart weight, grams (g); heart rate, beats per minute (bpm); systolic pressure, millimeters of mercury $(\mathrm{mmHg})$; coronary artery diameter, millimeters $(\mathrm{mm})$.

NHP have significantly contributed to the exploration of CVD and the development of effective therapies. However, there are still open questions about their genetics, metabolism, and physiology. These need to be addressed to increase the impact of these animal models in the field and to choose the optimal NHP species to address a particular research question.

\section{Genome editing}

Genome modification has been one of the major research goals in the last two decades. Currently, there are three major approaches for genome editing: Zinc finger nucleases (ZFNs), Transcription activator-like effector nuclease (TALENs), and Clustered Regularly Interspaced Short Palindromic Repeats (CRISPR). ZFNs consist of zincfinger transcription factors, each recognizing three base pairs attached to a restriction enzyme (usually FokI). The complexity of the design and construction makes this method challenging to use. TALENs are restriction enzymes (also usually FokI) with sequence-specific DNA binding domains that can be engineered to target a specific DNA sequence. While being highly specific, this system presents some disadvantages, for example, its incapability to target inactive chromatin (DNA methylation/histone acetylation) ${ }^{122}{ }^{123}$. First described in E. coli and S. epidermidis, the CRISPR/Cas system was discovered as an adaptive immune system in bacteria ${ }^{124}$. The modified CRISPR/Cas consists of two components, 1- a Cas9 nuclease (with two nuclease domains) that cuts the DNA, and 2- guide RNAs (gRNAs) with two functions: binding to Cas9 and recognition of a specific DNA sequence by base-pairing with the desired sequence in the chromosome. 
ZFNs, TALENs, and CRISPR generate double-strand breaks (DSB) that trigger the activation of the endogenous DNA reparation machinery. DNA can be repaired by two different means: non-homologous end joining (NHEJ) or homology-directed repair (HDR). In NHEJ the cell joins the two DNA ends after a DSB, while adding or deleting randomly base pairs (INDEL mutations). In contrast, in HDR the DNA damage is repaired precisely using an exogenous (extra-chromosomal) DNA template and no random mutations are induced ${ }^{123}$.

CRISPR, due to its simplicity and efficiency, is nowadays the preferred genome editing tool. The increasing sophistication of the CRISPR/Cas system together with refinement of iPSC generation makes it a feasible approach for research into genetic, molecular, and cellular mechanisms ${ }^{123}$. Besides the double nick activity, CRISPR/Cas enhancement makes possible using modified Cas9 for 1- single-strand nick (Cas9 partially inactive), or 2-blocking transiently expression of a gene (Cas9 inactive, no nickase activity) ${ }^{123}$.

The improvements of the CRISPR/Cas system tremendously increased its potential in biotechnological applications. Improved specificity and efficiency allows genomic targeting in any region, playing into the generation of genetically modified animals and cell lines. Furthermore, it brings the possibility of mutation corrections in genetically based diseases.

In conclusion, the combination of the versatility of PSCs, new genome editing tools and preclinically relevant (animal) models is revolutionizing our understanding of human diseases and helping to develop new treatments. In this study, we develop NHP-iPSCs which can be used to explore the potential of NHP for preclinical testing of regeneration therapies and for CVD modeling. 


\section{Aims of the thesis}

In this dissertation, I have explored the potential of NHP-PSC to be used 1- in stem cell-based regeneration therapies in vivo and, 2- to model CVDs. In the first part of the thesis, we aimed to improve the methods for NHP-iPSC generation, culture, and differentiation. Refined and highly standardized protocols have been established. In the second part, we focussed on overcoming the practical difficulties of applying CRISPR/Cas technologies to induce defined INDELs and deletion mutations in clonal NHP-iPSCs. Our final aim is to induce patient-specific mutations in NHP in vivo. To validate these approaches in vitro, it will be expedient to test the induction and the effect of predefined genetic modifications in species-specific iPSCs. Finally, based on the in silico analysis of the giant human sarcomeric protein Titin, we explored new therapeutic approaches for titinopathies.

1 st Hypothesis: Reprogramming of NHP and human fibroblasts can be achieved using a universal protocol, resulting in transgene- and feeder-free iPSC lines.

$-\mathbf{1}^{\text {st }}$ General objective: Refine methods for NHP-iPSCs generation.

a) Specific objective: Apply the previously published reprogramming method 59 based on the piggyBac transposon delivery of the reprogramming factors, to the baboon and rhesus macaque fibroblasts.

b) Specific objective: Generate human and quasi clinical-grade NHP-iPSCs by a universal protocol applicable to all NHP species included in this study.

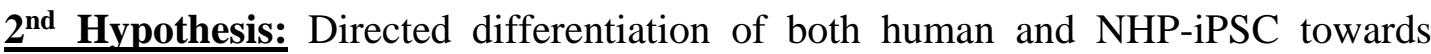
functional cardiomyocytes can be done using a single protocol.

$-2^{\text {nd }}$ General objective: Test currently available protocols for the generation of human iPSC-derived cardiomyocytes for the derivation of cardiomyocytes from NHP-iPSCs.

Specific objective: Evaluate the applicability and efficiency of human cardiomyocyte differentiation protocols in macaque, baboon and marmoset iPSCs.

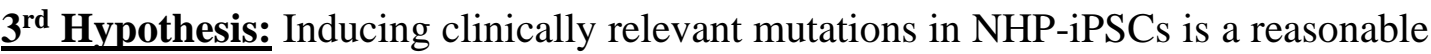
test system for a genetically modified in vivo NHP model of a monogenic CVDs.

$\mathbf{- 3}^{\text {rd }}$ General objective: Model cardiovascular disease in NHP-iPSCs.

a) Specific objective: Adapt the protocols for CRISPR based genomic modifications to the molecular particularities of NHP-iPSCs.

b) Specific objective: Induce clinically relevant mutations in NHP-iPSCs to model CVD. 
c) Specific objective: Analyse possible phenotypic alterations in the mutated iPSC derived cardiomyocytes. 


\section{Chapter 1: Baboon induced pluripotent stem cell generation by piggyBac transposition of reprogramming factors}

\section{Author contribution statement}

IRP and RB conceived and designed the experiments. IRP, MS, AB, and RD performed the experiments. IRP, IB, and RB analyzed the data. RD and IB designed and performed the teratoma assay and karyotyping, respectively. IRP and RB wrote the paper with contributions from all coauthors.

\begin{tabular}{|l|l|}
\hline \multicolumn{2}{|c|}{$\begin{array}{c}\text { Author contribution statement } \\
\text { by piggyBac transposition of reprogramming factors }\end{array}$} \\
\hline Table 1 & IRP performed the similarity analysis \\
\hline Figure 1 & $\begin{array}{l}\text { IRP, and AB during his bachelor thesis (under the } \\
\text { supervision of IRP) performed the reprogramming }\end{array}$ \\
\hline Figure 1 & IRP prepared the karyotypes, IB stained and analyzed the karyograms \\
\hline Figure 1, 2, 3, and 4 & $\begin{array}{l}\text { IRP performed the characterization of the generated lines } \\
\text { and the adaptation to feeder-free culture }\end{array}$ \\
\hline Figure 3 & MS adapt the Rhesus ESC to feeder-free conditions \\
\hline Figure 5, and Suppl. Figure 2 & $\begin{array}{l}\text { RD performed the teratoma formation assay } \\
\text { IRP, RB, and RD analyzed the teratoma histology }\end{array}$ \\
\hline Experiment design & IRP and RB \\
\hline Manuscript writing & IRP and RB, with the contribution of all authors \\
\hline
\end{tabular}




\title{
Baboon induced pluripotent stem cell generation by piggyBac transposition of reprogramming factors
}

\author{
Ignacio Rodriguez-Polo ${ }^{1,2}$, Michael Stauske ${ }^{1,2, a}$, Alexander Becker ${ }^{1}$, Iris Bartels ${ }^{3}$, Ralf Dressel ${ }^{2,4}$, and \\ Rüdiger Behr ${ }^{1,2}$ \\ ${ }^{1}$ Research Platform Degenerative Diseases, German Primate Center - Leibniz Institute for Primate Research, \\ Kellnerweg 4, 37077 Göttingen, Germany \\ ${ }^{2}$ German Center for Cardiovascular Research (DZHK), Partner site, Göttingen, Germany \\ ${ }^{3}$ Institute of Human Genetics, University Medical Center Göttingen, Robert-Koch-Str. 40, \\ 37075 Göttingen, Germany \\ ${ }^{4}$ Institute of Cellular and Molecular Immunology, University Medical Center Göttingen, \\ Robert-Koch-Str. 40, 37075 Göttingen, Germany \\ ${ }^{a}$ current address: BlueRock Therapeutics, 101 College St, PMCRT 14-301, Toronto, ON M5G 1L7, Canada
}

Correspondence: Rüdiger Behr (rbehr@dpz.eu)

Received: 11 April 2019 - Revised: 30 June 2019 - Accepted: 1 July 2019 - Published: 29 July 2019

\begin{abstract}
Clinical application of regenerative therapies using embryonic or induced pluripotent stem cells is within reach. Progress made during recent years has encouraged researchers to address remaining open questions in order to finally translate experimental cell replacement therapies into application in patients. To achieve this, studies in translationally relevant animal models are required to make the final step to the clinic. In this context, the baboon (Papio anubis) may represent a valuable nonhuman primate (NHP) model to test cell replacement therapies because of its close evolutionary relationship to humans and its large body size. In this study, we describe the reprogramming of adult baboon skin fibroblasts using the piggyBac transposon system. Via transposon-mediated overexpression of six reprogramming factors, we generated five baboon induced pluripotent stem cell (iPSC) lines. The iPSC lines were characterized with respect to alkaline phosphatase activity, pluripotency factor expression analysis, teratoma formation potential, and karyotype. Furthermore, after initial cocultivation with mouse embryonic fibroblasts, we were able to adapt iPSC lines to feeder-free conditions. In conclusion, we established a robust and efficient protocol for iPSC generation from adult baboon fibroblasts.
\end{abstract}

\section{Introduction}

The development of successful approaches for the induction of pluripotency in somatic cells to generate induced pluripotent stem cells (iPSCs) was ground-breaking (Takahashi and Yamanaka, 2006). Pluripotent stem cells (PSCs) are promising for the treatment of degenerative diseases, which are associated with the progressive loss of functional cells in the patient. Regarding therapeutic application, iPSCs overcame the practical and ethical difficulties associated with the use of embryonic stem cells (ESCs) for cell replacement therapies (CRTs) (Takahashi et al., 2007). One therapeutic approach is to transplant iPSC-derived immature tissue-specific cells, such as neuro- or cardio-progenitor cells (Grow et al., 2016b). An alternative approach is the use of in vitro grown tissue, such as engineered heart muscle, generated from iPSC-derived cardiomyocytes cultured in three-dimensional tissue scaffolds (Tiburcy et al., 2017; Stevens et al., 2009; Turnbull et al., 2014).

The combined efforts of researchers resulted in a broad panel of reprogramming methods for the generation of iPSCs (Malik and Mahendra, 2013; Patel and Yang, 2010). This allows the exploration of different strategies for cell reprogramming, also impacting potential CRTs in humans (Sosa et al., 2017; Zhang et al., 2017). However, several aspects need to be addressed before routine clinical application including (i) long-term safety, (ii) survival and prevalence of 
the transplanted cells in the in vivo context of an immune system similar to humans, (iii) cell functionality after transplantation, and (iv) feasibility of the upscaling of the production of the cells and the size of the transplant. To achieve this, relevant preclinical animal models are required (Turnbull et al., 2014; Kobayashi et al., 2012; Grow et al., 2016a; Kimbrel and Lanza, 2015).

Within the spectrum of animal models suitable for translational research, nonhuman primates (NHPs) exhibit distinct advantages (Phillips et al., 2014; Behr, 2015). The similarities between humans and NHPs such as their genetic background, immune system, and anatomy make them an attractive model for the validation of new therapeutic strategies like CRTs (Grow et al., 2016a). Invasive biomedical research with great apes as the evolutionarily closest relatives of humans is restricted for ethical and legal reasons. Therefore and because of its relatively broad availability, rhesus macaques (Macaca mulatta) have been the NHP model of choice for many biomedical studies including neuroscience and HIV and AIDS research (Grow et al., 2016a; Navara et al., 2013; Didier et al., 2016). Although the rhesus macaque is a well-established model, the establishment of alternative models that share the advantageous characteristics of the rhesus macaque and exhibit specific additional advantages is desirable. The olive baboon (Papio anubis) is an Old World monkey with comparable evolutionary distance to humans as the macaques. It shares $92 \%$ of the genome with humans, has a life expectancy of up to 45 years, and very closely resembles human development, anatomy, and physiology (Navara et al., 2018; Bailey, 2009; Rogers and Hixson, 1997). In comparison to macaques and other NHP species except for great apes, baboons have (i) a more similar size and weight in comparison with humans (16.4-21.3 kg for males and 10 $15 \mathrm{~kg}$ for females) in contrast to rhesus (4-14.1 kg for males and $3-10 \mathrm{~kg}$ for females); (ii) they alternate quadrupedal and bipedal locomotion, and (iii) regarding transplantation studies, it is of relevance that their immune system closely resembles the human one, e.g., presenting the same four immunoglobulin $\mathrm{G}$ ( $\mathrm{IgG}$ ) subclasses (Grow et al., 2016a; Navara et al., 2018; Varilly and Chandler, 2008; Shearer et al., 1999). Furthermore, controlled-breeding colonies exist that have been studied for many years. Overall, baboons are an excellent NHP model for stem-cell-based transplantation studies (Navara et al., 2013; Bailey, 2009; Simerly et al., 2009; Agrba et al., 2016; Cox et al., 2013; Längin et al., 2018).

In parallel to addressing the remaining open questions of CRTs in animal models, the last years have seen an evolution of reprogramming approaches for the generation clinical grade iPSC (Malik and Mahendra, 2013; Patel and Yang, 2010; Kimbrel and Lanza, 2015). Virus-based reprogramming is very robust and efficient but poses a drawback for clinical application. The original retroviral reprogramming approach is associated with the proviral integration of the vector into the host cell genome and an increased oncogenic potential that is incompatible with the clinical use of respective iPSCs (Kimbrel and Lanza, 2015; Kim et al., 2012). Therefore, non-integrative (non-mutagenic) reprogramming approaches have been developed for human and mouse cells (Malik and Mahendra, 2013; Yu et al., 2009; Yamanaka et al., 2011). Recently, some of them have been adapted to macaques. For example, rhesus macaque iPSCs were generated from embryonic fibroblasts using self-replicating RNA engineered from the Venezuelan equine encephalitis (VEE) virus, which has a positive-strand RNA genome (Sosa et al., 2017). Also, episomal vectors have been used to generate iPSCs from macaque postnatal ear skin fibroblasts (Zhang et al., 2017). However, in both cases, this was achieved under feeder-dependent conditions. To test CRT preclinically in NHP, it is required to cultivate the cells under feederfree conditions. Cultivation of iPSC using feeder cells, e.g., mouse embryonic fibroblasts (MEFs) or human foreskin fibroblasts (HFFs), limits mass-production and puts at risk the purity of the potential iPSC-derived graft (Villa-Diaz et al., 2013; Nishizawa et al., 2014; Hakala et al., 2009). Furthermore, it is important to have the option to generate vectorfree iPSCs in order to preclinically test applications in humans (Shiba et al., 2016; Emborg et al., 2013; Wang et al., 2015).

In contrast to the macaque, the baboon has been widely neglected as an animal model for CRT testing, with very few exceptions (Simerly et al., 2009). Navarra and colleagues generated the first baboon iPSC (biPSC) using a retroviral approach (Navara et al., 2013). Recently this group also reprogrammed baboon peripheral blood cells using a nonintegrating Sendai virus approach under feeder cell conditions (Navara et al., 2018). For thorough testing of the potential of CRT, however, it is necessary to have a broad panel of different cell lines available as inter iPSC line variability is common (Nishizawa et al., 2016; Carcamo-Orive et al., 2017; Ohi et al., 2011). Hence, it is important to generate different baboon iPSC lines by different reprogramming approaches and from different sources. This will allow the identification of the general and specific characteristics of baboon iPSC lines, which is important regarding the interpretation of preclinical data generated using NHPs and their translation into the clinics (Didier et al., 2016; Cox et al., 2013).

The piggyBac system has been shown to be useful for generating human, mouse, and marmoset monkey iPSCs (Debowski et al., 2015; Woltjen, 2011; Yusa et al., 2009; Mohseni et al., 2009). The piggyBac transposon-based approach represents several advantages such as (i) the robustness of the expression of the reprogramming factors, (ii) the capacity to deliver large DNA fragments, and (iii) the possibility of footprint-free excision of the vector after reprogramming. Using a six-factor-in-one vector transposon, we reported the successful reprogramming of marmoset monkey (Callithrix jacchus) postnatal skin fibroblasts into iPSCs (Debowski et al., 2015). 
In this study, we adapted our established piggyBac reprogramming protocol to baboon cells in order to contribute to establishing this species as a preclinical model for CRTs. Employing the nonviral piggyBac approach, we were able to reproducibly reprogram baboon adult skin fibroblasts. We also managed to adapt baboon iPSC lines from feederdependent to feeder-free culture conditions, which will allow the xeno-free mass production of biPSCs.

\section{Results}

\subsection{Baboon skin fibroblast reprogramming by the piggyBac transposition}

Five independent biPSC lines, termed DPZ_biPSC1 to DPZ_biPSC5, were derived from fibroblasts isolated from a skin biopsy of an adult female baboon. Reprogramming was performed using our previously published piggyBac six-factor transposon system, containing the marmoset SOX2, OCT4, KLF4, c-MYC, NANOG, and LIN28 sequences (Fig. 1a). In silico similarity analyses between the six reprogramming factors of the marmoset and the respective baboon sequences showed evolutionary conservation (>96\%); only NANOG was less conserved (Table 1). Between days 25-30 after nucleofection, 10 to 15 primary colonies per plate (approx. 100 in total) with a morphology distinct from the non-reprogrammed fibroblasts were identified; new colonies appeared until day 60 . Considering the number of colonies identified in this experiment, the overall reprogramming efficiency in this experiment is $0.0001 \%$ ( $\sim 100$ colonies $/ 1 \times 10^{6}$ transfected cells during the course of the experiment - more colonies could have developed after termination of the reprogramming period) in relation to the total fibroblast transfected and $0.0063 \%$ (12.5 colonies per primary plate $/ 0.2 \times 10^{5}$ cells per primary plate) in relation to the puromycin resistant cells. Colonies had a stable morphology independent of the passage number and resembled other primate iPSCs in feeder culture. The colonies presented clear borders, and compact structure, and the cells had a high nuclei/cytoplasm ratio (Fig. 1a). During the first passages (approx. until passage 5) colonies with good morphology were picked manually to stabilize the different lines in culture. Then five colonies meeting the morphological PSC criteria were selected and further passaged and characterized (Fig. 1a). As a first screen for pluripotency, alkaline phosphatase activity was evaluated between passage 10 and 15. Generated iPSCs lines show alkaline phosphatase (AP) activity (Fig. 1b). Karyotyping was performed for biPSC1 and biPSC4. The cytogenetic analysis revealed a numerically normal karyotype of a female baboon without any evidence of structural chromosomal abnormalities (Fig. 1c).
Table 1. Similarity of the six factors used for reprogramming between marmoset and baboon.

\begin{tabular}{lrr}
\hline & cDNA $(\%)$ & Protein $(\%)$ \\
\hline SOX2 & 94.06 & 99.37 \\
OCT4 & 85.37 & 96.03 \\
KLF4 & 87.54 & 100 \\
LIN28 & 96.98 & 98.56 \\
c-MYC & 82.84 & 97.72 \\
NANOG & 84.75 & 88.59 \\
\hline
\end{tabular}

\subsection{Detection of pluripotency markers}

Each of the five lines generated expressed well-established pluripotency markers, including OCT4A (POU5F1), LIN28, TRA-1-60, TRA-1-81, SALL4, SOX2, and NANOG, as tested by immunostaining (Fig. 2). OCT4A, SALL4, NANOG, and SOX2 are nuclear, while LIN28 was detected in the cytoplasm. TRA1-60 and TRA-1-81 show membrane and cytoplasmic staining. These data indicate successful reprogramming (Fig. 2). Isotype controls were performed as a negative control (Fig. S1 in the Supplement).

We performed reverse transcription polymerase chain reaction (RT-PCR) to corroborate the expression of these factors at the messenger RNA (mRNA) level and to discriminate between endogenous expression and exogenous expression from the transposon (Fig. 3). To assess the expression of the transposon-derived transcript, primers were designed to demonstrate the presence of the $5^{\prime}$-end of the synthetic transposon transcript covering the fused SOX2-OCT4 sequences, and at the 3 '-end LIN28-NANOG (Fig. 3a and b). The transcript analysis was performed at passage 15 to 30 . None of the lines showed the absence of the exogenous transcript according to the semiquantitative RT-PCR analysis. Although the piggyBac expression is still active, the biPSC lines present a comparable expression of endogenous OCT4A, ZFP42, and SOX2 to rhesus monkey embryonic stem cells (rhESCs), which were used in the absence of a baboon ESC line as a positive control (Fig. 3). Only the intensity of the NANOG band was considerably weaker in some baboon iPSC lines than in the rhESC line.

\subsection{Feeder-dependent and feeder-free culture of iPSCs}

Three biPSC lines (DPZ_biPSC1, 4, and 5) were adapted to feeder-free conditions. The cells proliferated and remained undifferentiated in standard human iPSCs culture conditions, using Geltrex as an attachment matrix and Essential 8 medium (Fig. 4). The morphology of the colonies changed during the process of adaptation, and the lines stabilized after about three passages after the switch to the new culture conditions. Although adaptation of all five biPSC to feederfree conditions was tested, only three of the lines were stable in the new culture conditions. The lines under feeder-free 
(a)
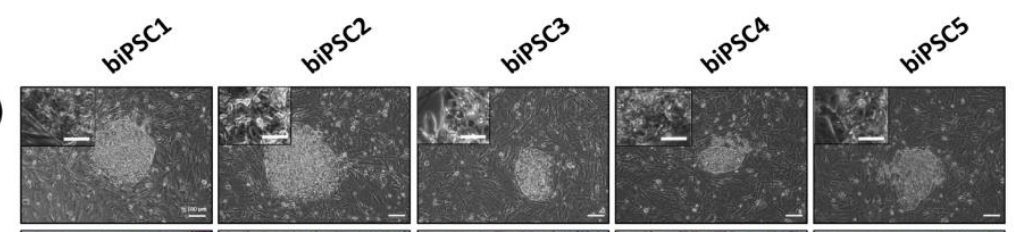

(b)

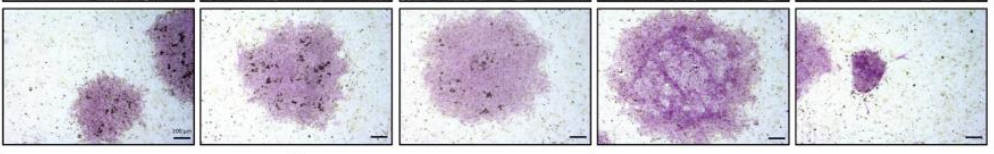

(c)

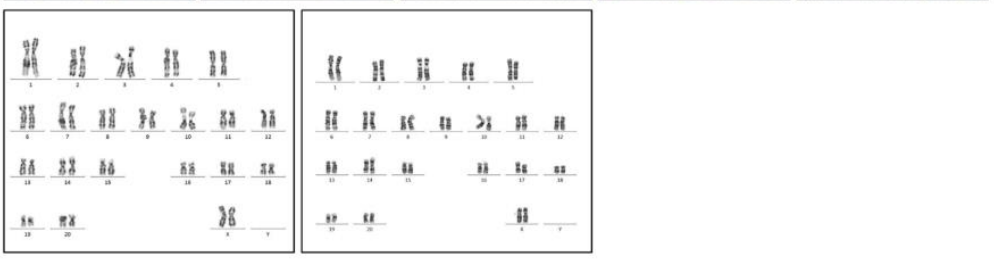

Figure 1. Baboon induced pluripotent stem cells. (a) iPSCs morphology of the five baboon lines generated and characterized (DPZ_biPSC1 to DPZ_biPSC5; scale bars 100 and $25 \mu \mathrm{m}$, inset). (b) Alkaline phosphatase staining (scale bar $200 \mu \mathrm{m}$ ). (c) Representative G-banded female karyotypes, female karyotypes (42; XX) of DPZ_biPSC1 (left karyogram) and DPZ_biPSC4 (right karyogram).

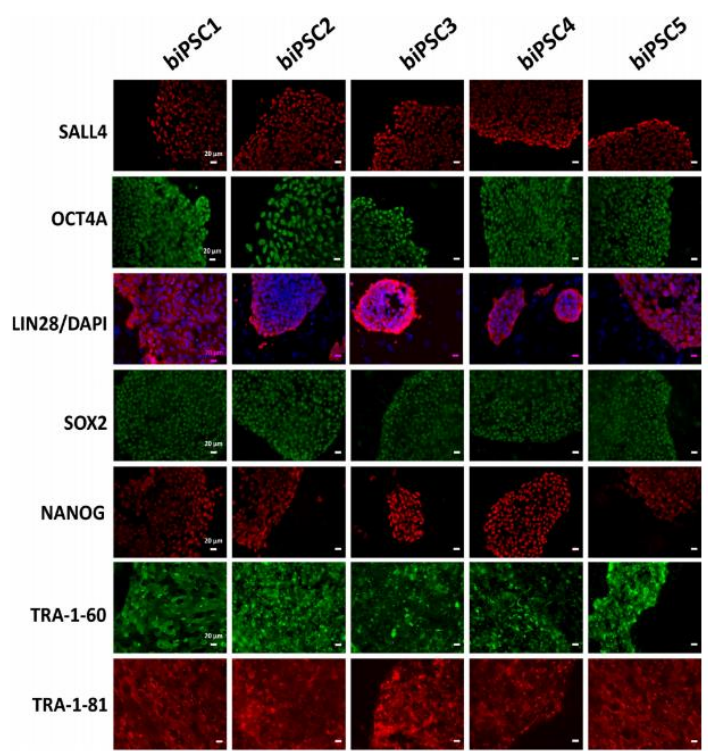

Figure 2. biPSC characterization by immunofluorescence staining. Detection of the pluripotency markers SALL4, OCT4A, LIN28, SOX2, NANOG, TRA-1-81, and TRA-1-60and. OCT4A, SOX2, NANOG, and LIN28 expression comes from both endogenous gene reactivation and exogenous transposon expression. SALL4, TRA-181, and TRA-1-60 result exclusively from endogenous expression as they are not contained in the reprogramming vector (scale bars $20 \mu \mathrm{m})$.

Primate Biol., 6, 75-86, 2019 conditions maintained the typical cellular and colony morphology, i.e., small cells with a high nuclei/cytoplasm ratio. The colony morphology changed slightly in the new conditions; the rather regular borders seen on feeders changed to a more fringy appearance (Fig. 4a, also compare with Fig. 1a). After obtaining a homogenous undifferentiated morphology throughout the culture plate, it was possible to passage the cells by gentle dissociation using Versene solution instead of manual picking (Fig. 4a). Splitting using collagenase type IV and accutase was tested but led to a higher degree of morphological differentiation (data not shown). Five to ten passages after adaptation, a basic characterization was performed to check if the iPSCs remained undifferentiated. Expression of NANOG and OCT4A as pluripotency-specific markers as well as TRA-1-81 and AP indicated that the cells remained pluripotent even under feeder-free conditions (Fig. 4a-e).

\subsection{Baboon iPSC differentiation by teratoma formation}

To demonstrate the differentiation potential of the generated iPSCs functionally, the teratoma formation assay was exemplarily performed for the DPZ_biPSC lines 1 and 5 by injecting $\sim 1 \times 10^{6}$ iPSCs subcutaneously into immunodeficient mice. DPZ_biPSC1 was injected after adaptation to feeder-free conditions, in contrast to DPZ_biPSC5, which remained under feeder-dependent conditions until injection. Tumors developed within 15 weeks. According to the type of culture (with MEFs or feeder-free on Geltrex), the lines were injected together with MEFs (DPZ_biPSC5) or Geltrex (DPZ_biPSC1) as an initial support for survival after injection (Fig. 5). 


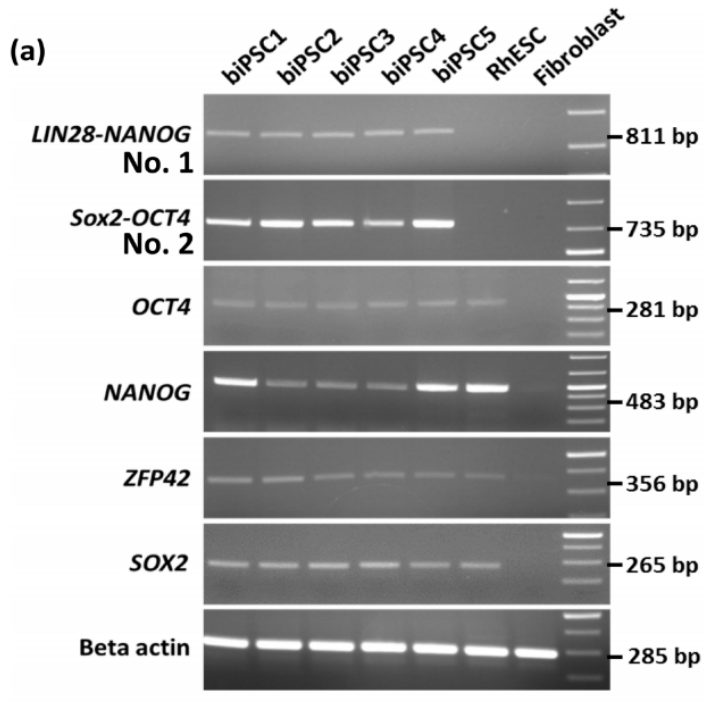

(b)

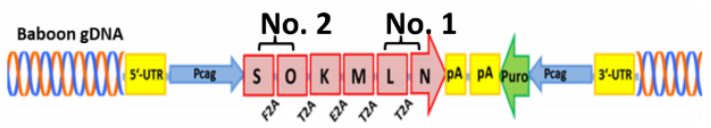

(c)

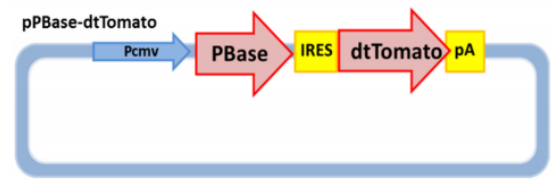

Figure 3. biPSC characterization by RT-PCR. (a) Expression analysis of the five baboon iPSCs lines (DPZ_biPSC1-5). In the absence of a baboon ESC line, rhesus embryonic stem cells (rhESC) were used as a positive control and baboon primary fibroblasts as a biological negative control (fibroblast). A $1 \mathrm{~Kb}$ Plus DNA Ladder was used as size marker. RT-PCR analysis of the iPSCs lines for the endogenous pluripotency factors OCT4A, NANOG, ZFP42, and SOX2 as well as for the reprogramming cassette expression at two different sites, namely SOX2-OCT4 (beginning of the transposon; no. 2 see b), LIN28-NANOG (end of the transposon; no. 1). (b) piggyBac transposon used in this study, coding for the marmoset (Callithrix jacchus) reprogramming factors, SOX2 (S), OCT4 (O), KLF4 (K), c-MYC (M), LIN28 (L), and NANOG (N). 2A peptides substituting the stop codons were inserted between the reprogramming genes. The expression of the factors is driven by a CAG promoter. The puromycin resistance gene (Puro) is expressed under the control of an independent CAG promoter. (c) The pBase-dtTomato construct was transiently co-expressed with the vector shown in (b) and facilitates the integration of the transposon in the genome. Transposase expression is driven by the cytomegalovirus promoter $(\mathrm{Pcmv})$.
Both lines formed tumors that were analyzed when their diameter reached approximately $1 \mathrm{~cm}$ (Figs. 5a and S2a). The expression of markers of each embryonic germ layer was immunohistochemically analyzed. In a DPZ_biPSC1-derived tumor SOX9 (primitive endodermal epithelium), $\beta$-tubulin 3 (ectoderm), and smooth muscle actin (SMA) (mesoderm) were detected, demonstrating the pluripotency of this line (Fig. 5c-e). Also, clusters of pigmented cells were detected (Fig. 5f), which also indicate ectoderm. The DPZ_biPSC5derived teratoma similarly showed differentiated cells expressing the respective markers but also contained clusters that remained undifferentiated as indicated by OCT4A(Fig. S2c) and NANOG-positive (not shown) cells, even 15 weeks after injection.

\section{Methods}

\subsection{Animals and animal housing; ethics statement}

The German Primate Center is registered and authorized by the local and regional veterinary governmental authorities (reference number: 122910.3311900, PK Landkreis, Göttingen). The DPZ runs self-sustaining colonies of different primate species including baboons. The animal from which the cells were obtained was sacrificed at the age of 12 years in the context of an unrelated approved study. Skin samples were made available during necropsy to the Platform Degenerative Diseases of the DPZ.

\subsection{Cell culture}

\subsubsection{Isolation of baboon primary fibroblasts}

Skin tissue pieces of approximately $1 \times 1 \mathrm{~cm}$ were dissected to remove subcutaneous adipose tissue and washed three times with phosphate-buffered saline (PBS) buffer with antibiotics $(1 \%, v / v$, penicillin/streptomycin, Gibco, $0.25 \mu \mathrm{g} \mathrm{mL}^{-1}$ amphotericin B, Sigma). The skin was minced with scalpels and digested in Dulbecco's Modified Eagle Medium (DMEM) supplemented with $10 \mathrm{mg} \mathrm{mL}^{-1}$ collagenase type IV (Gibco) for $3 \mathrm{~h}$ at $37^{\circ} \mathrm{C}$ at a constant rotation at $800 \mathrm{rpm}$. After the digestion, the remaining nondigested skin fragments were removed, and the cell suspension was centrifuged $(300 \mathrm{~g}, 5 \mathrm{~min}$, RT). The supernatant was discarded, and the pellet resuspended in Rh15 medium (DMEM, Gibco, $15 \%, v / v$; fetal bovine serum, Gibco, $1 \%$, $v / v$; penicillin/streptomycin, Gibco, $0.25 \mu \mathrm{g} \mathrm{mL}^{-1}$; amphotericin B, Sigma, $1 \%, v / v$; Minimum Essential Medium (MEM) non-essential amino acids solution, Gibco; $2 \mathrm{mM}$ GlutaMAX, Gibco) and plated onto two $10 \mathrm{~cm}$ diameter gelatine-coated culture dishes ( $0.1 \%$ gelatine; Sigma). Fibroblasts were passaged using StemPro accutase (Thermo Fisher). 

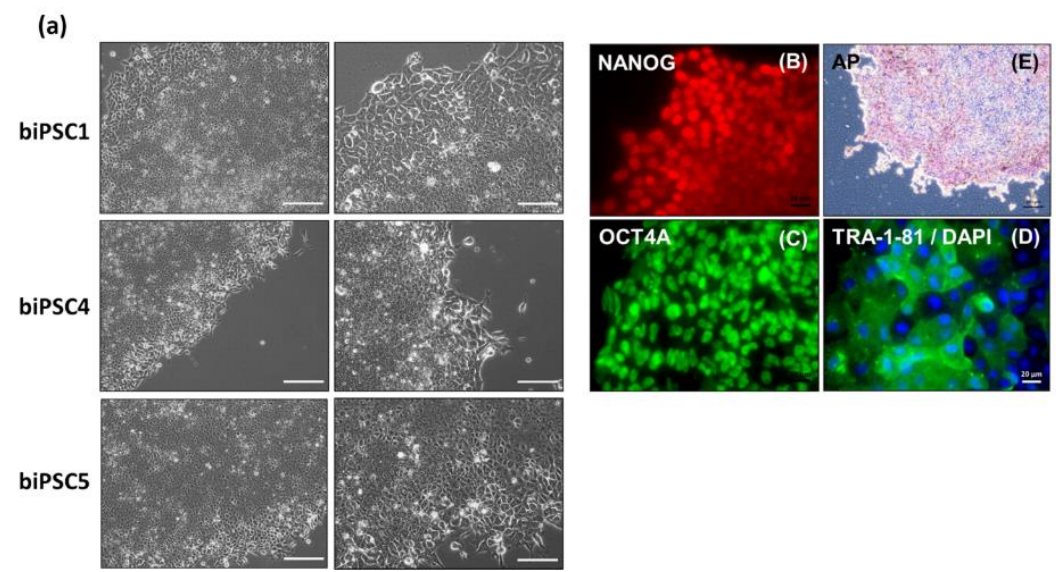

Figure 4. biPSCs adaptation to feeder-free conditions. (a) Bright field pictures of DPZ_biPSC1, DPZ_biPSC4, and DPZ_biPSC5 in culture on Geltrex with E8 medium (scale bar, a $200 \mu \mathrm{m}$; b-d $20 \mu \mathrm{m}$ and e $100 \mu \mathrm{m}$ ). (b-d) DPZ_biPSC1 immunofluorescence staining, for (b) NANOG, (c) OCT4A, and (d) TRA1-81 to confirm that the lines remain undifferentiated after the adaptation to the feeder-free conditions (scale bar $20 \mu \mathrm{m}$ ). (e) Alkaline phosphatase staining of DPZ_biPSC1 under feeder-free conditions (scale bar represents $100 \mu \mathrm{m}$ ).
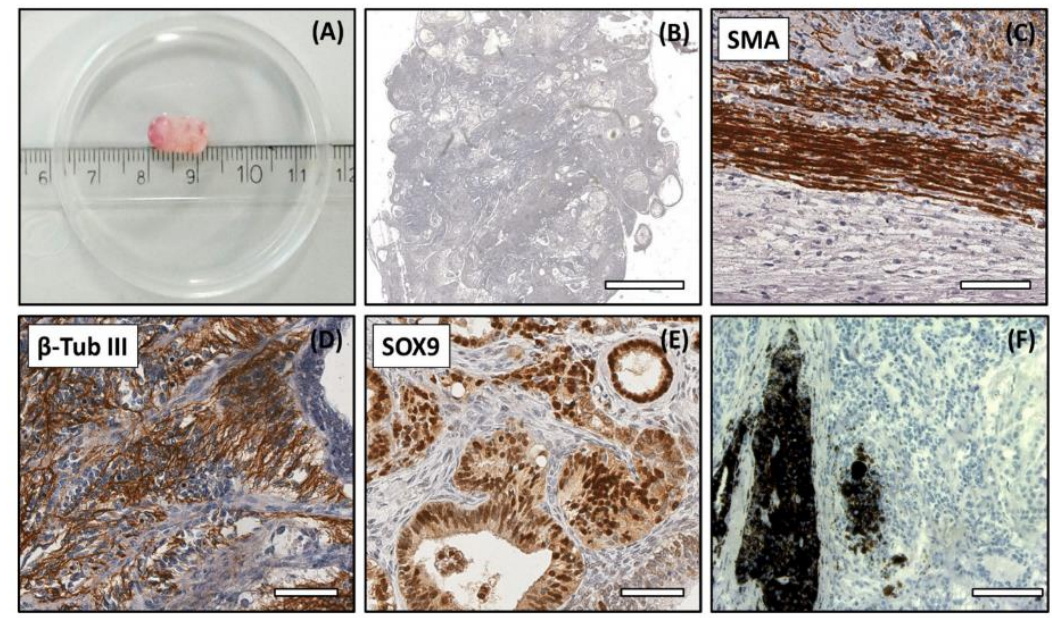

Figure 5. Analysis of the DPZ_biPSC 1 teratoma. Tumor tissue was immunohistochemically analyzed for the expression of representative markers of the three germ layers: SOX9, $\beta$-tubulin 3, and smooth muscle actin (SMA). (a) Macroscopic view of DPZ_biPSC 1 teratoma. (b) Overview of a H\&E-stained teratoma section showing the structural heterogeneity (bar represents $2 \mathrm{~mm}$ ). (c) Smooth muscle actin (SMA) staining indicating mesodermal differentiation. (d) $\beta$-Tubulin 3 staining ( $\beta$-Tub III) indicating ectodermal differentiation. (e) SOX9 staining. SOX9 is a marker of endodermal progenitor and stem cells. (f) Pigmented cells (no immunohistochemical staining) indicating ectodermal differentiation (bars in c-f represent $70 \mu \mathrm{m}$ ).

\subsubsection{Mouse embryonic fibroblasts (MEFs)}

Gamma-irradiated MEFs were used as feeder cells. Their generation and culture were described previously (Debowski et al., 2015).

Primate Biol., 6, 75-86, 2019

\subsubsection{Nucleofection}

For reprogramming the piggyBac NHP six-factor transposon system was used (Debowski et al., 2015). Baboon fibroblasts $\left(1 \times 10^{6}\right.$ cells $)$ were nucleofected $(6 \mu \mathrm{g}$ pDNA $)$ after at least three passages in culture using the 4-D Nucleofector (Lonza), with P2 Primary Cell 4D-Nucleofector ${ }^{\circledR}$ X Kit L (Lonza; program CA-137). A pmax-GFP (green fluorescent protein) vector was used as a positive control for nucleofection.

www.primate-biol.net/6/75/2019/ 


\subsubsection{Reprogramming procedure}

After nucleofection, the efficiency was estimated by the expression of the reporters in the pBase-dtTomato transposase (Fig. 3c) and the pmax-GFP. Antibiotic selection was started $2 \mathrm{~d}$ after nucleofection by supplementation of the Rh15 medium for $5 \mathrm{~d}$ with $1.5 \mu \mathrm{g} \mathrm{mL}^{-1}$ puromycin (Sigma). After selection, the cells were transferred at day 6 after nucleofection onto gelatine-coated $10 \mathrm{~cm}$ plates with MEFs $\left(0.2 \times 10^{5}\right.$ cells per plate) and cultured in embryonic stem cell medium (ESM) (KO-DMEM, Gibco; $20 \%$, $v / v$, KnockOut Serum Replacement, Gibco; $1 \%, v / v$, penicillin/streptomycin, Gibco; $0.25 \mu \mathrm{g} \mathrm{mL}^{-1}$ amphotericin B, Sigma; $1 \%, v / v$, MEM nonessential amino acid solution, Gibco; 2 mM GlutaMAX, Gibco; $50 \mu \mathrm{M} 2$ 2-mercaptoethanol, Gibco) supplemented with $10 \mathrm{ng} \mathrm{mL}^{-1}$ fibroblast growth factor (FGF, ThermoFisher). During the first $6 \mathrm{~d}$ of culture on MEFs, ESM was supplement with $2 \mathrm{mM}$ valproic acid (Calbiochem). The first colonies appeared at day 25 , and new colonies were picked until day 60 . Colonies were picked manually from the primary plate to fresh plates with MEFs. Around passage 5, colonies were stable enough to stop manual picking and passaged using $1 \mathrm{mg} \mathrm{mL}^{-1}$ collagenase type IV (Gibco).

Freezing of the cells was performed using ESC medium with $20 \%$ DMSO (dimethylsulfoxid, DMSO; Sigma at $\left.-150^{\circ} \mathrm{C}\right)$.

\subsection{RT-PCR to evaluate the expression of endogenous} and exogenous (piggyBac) pluripotency factors

RNA extraction was performed from frozen cell pellets using the RNAeasy Mini Kit (Qiagen). For complementary DNA (cDNA) synthesis, the omniscript RT kit (Qiagen) was used according to the manufacturer's protocol using $1 \mu \mathrm{g}$ mRNA per reaction. Residual genomic DNA (gDNA) was removed using the RNase-Free DNase kit (Qiagen). Oligonucleotides (Sigma) are detailed in Table 2. Primers to analyze endogenous expression were designed specifically for the $3^{\prime}$ UTR of each pluripotency gene, which is not included in the reprogramming cassette. Specific amplification of exogenous transcripts was performed by designing primers located in two consecutive coding sequences of the piggyBac not present in the wild type genome. In the absence of baboon ESCs, rhesus monkey embryonic stem cells (rhESC; line 366.4) (Thomson et al., 1995) were used as a positive control and baboon fibroblasts as a negative control. Beta-actin was used as a housekeeping gene for normalization.

\subsection{Alkaline phosphatase}

Alkaline phosphatase activity was demonstrated using the leukocyte alkaline phosphatase kit (Sigma) according to the manufacturer's instructions.
Table 2. Oligonucleotides (Sigma) used for the cDNA amplification of the endogenous and exogenous pluripotency factors and $A C T B$ used as housekeeping gene.

\begin{tabular}{ll}
\hline Name & ${\text { Primer sequence } 5^{\prime} 3^{\prime}}^{\prime}$ \\
\hline SOX2-OCT4_fw & TCTTCCTCGCACTCCAGGGC \\
SOX2-OCT4_rev & CAGGGTGATCCTCTTCTGCTTC \\
LIN28-NANOG_fw & AGCCATATGGTAGCCTCATGTCC \\
LIN28-NANOG_rev & GGTTGCTCCAGGTTGAATTGC \\
OCT4_fw & GAAGGATGTGGTCCGAGTGT \\
OCT4_rev & AGGCGCCTCAGTTTGAATGC \\
NANOG_fw & GCAATGGTGTGACTCAGAAGG \\
NANOG_rev & TTCAGCCAGTGCCCAGACTG \\
ZFP42_fw & CAGAGCCCATTCTTGGTCGG \\
ZFP42_rev & CATTTCCGCCTGCAGGTCTT \\
SOX2_fw & GGCATGGCTCTTGGTTCCAT \\
SOX2_rev & TGTCCTGCCCTCACATGTGC \\
ACTB_fw & GACCTGACTGACTACCTCATG \\
ACTB_rev & GGTAGTTTCGTGGATGCCACA \\
\hline
\end{tabular}

\subsection{Immunofluorescence staining}

Cells were cultured on coverslips until the colonies reach $60 \%-80 \%$ confluence. For fixation, the plates were washed three times with PBS and fixed using $4 \%$ paraformaldehyde (PFA) $(v / v)$ for $20 \mathrm{~min}$ at room temperature. Before blocking, cells were washed three more times with PBS. Blocking was performed using $1 \%$ bovine serum albumin (BSA) in PBS for surface markers or $1 \%$ BSA in PBS supplemented with TritonX-100 (0.1\%, Sigma) for permeabilization in the case of staining intracellular markers. The primary antibody was diluted in 1\% BSA in PBS and the cells were incubated for $1 \mathrm{~h}$ at $37^{\circ} \mathrm{C}$. The washing step described above was repeated between first and second antibody incubations. Alexa488-coupled secondary antibodies (Life Technologies) diluted in $1 \%$ BSA in PBS were also applied for $1 \mathrm{~h}$ at $37^{\circ} \mathrm{C}$. Cells were finally incubated in $4^{\prime}, 6$-diamidino2-phenylindole (DAPI) diluted in PBS $\left(05 \mu \mathrm{gL}^{-1}\right.$; Roth) for $10 \mathrm{~min}$ to stain the nuclei. The coverslips were mechanically detached and mounted using citifluor mountant medium (CITIFLUOR ltd.). Immunofluorescence images were taken with a Zeiss Observer Z1 (Zeiss). The primary antibodies used and their dilutions were OCT4A (cell signalling OCT4A C52G3, $1: 1600$ ), SOX2 (cell signalling C70B1, $1: 200$ ), NANOG (cell signalling D73G4, $1: 400$ ), TRA 1-60 (eBioscience 14-8863, $1: 100$ ), TRA 1-81 (eBioscience 14-8883, $1: 100$ ), LIN28 (cell signalling A177, $1: 100$ ), and SALL4 (Abcam ab57577, 1:200). As secondary antibodies, Alexa488 conjugated donkey anti-mouse IgG $(\mathrm{H}+\mathrm{L})$ (Life technologies A21202, $1: 1000)$, donkey anti-rabbit $\operatorname{IgG}(\mathrm{H}+\mathrm{L})$ (Invitrogen A-21206, $1: 1000$ ), and goat anti-mouse immunglobulin M (IgM) $(\mathrm{H}+\mathrm{L})$ (Invitrogen A-21042, $1: 1000)$ were used. 


\subsection{Teratoma formation and histological analysis}

For the teratoma assay, cells were prepared for injection by mixing $8 \times 10^{5}$ biPSC with $2 \times 10^{5}$ MEFs in the case of injection with feeders and $1 \times 10^{6}$ biPSCs in the case of feederfree lines. Cells were diluted in PBS substituted with Geltrex solution $\left(0.1 \mathrm{mg} \mathrm{mL}^{-1}\right.$, ThermoFisher) with a final volume of $120 \mu \mathrm{L}$ per injection. Immunodeficient $\mathrm{RAG}^{-/-} \gamma \mathrm{c}$ $-/-$ mice were injected subcutaneously with the different cell lines. The mice were checked regularly, and the formation of tumors was monitored by palpation. Teratomas were isolated when reaching a diameter of $1 \mathrm{~cm}$ around 15 weeks after the injection. The immunohistochemical staining protocol has been described previously (Eildermann et al., 2012). The antibodies used for immunohistochemical staining were anti$\beta$-tubulin III (Sigma, T8660, $1: 600$ ), anti-SMA (Sigma, A2547, $1:$ 1000), anti-SOX9 (Millipore, AB5535, $1: 1000$ ), and anti-OCT4A (cell signalling, 2890, $1: 1000$ ).

\subsection{Adaptation to feeder-free conditions}

Baboon iPSC colonies were manually dissociated and transferred using a pipet to a $7 \mathrm{~cm}$ culture dish coated with Geltrex (Thermo Fisher Scientific). Cells were cultured in Essential 8 medium (E8; Thermo Fisher). For the first $2 \mathrm{~d}$ after the transfer, the medium was supplemented with ROCK inhibitor pro-survival compound (PSC DDD00033325 - Calbiochem; $5 \mu \mathrm{M}$ ). Colonies were initially manually picked and then split with Versene solution (Thermo Fisher). Dilution factors during passaging of the cells varied between $1: 10$ and $1: 20$. Freezing of the cells under feeder-free conditions was done using the Essential 8 medium supplemented with $20 \%$ DMSO (Sigma) and $10 \mu \mathrm{M}$ PSC.

\subsection{Karyotyping}

Baboon iPSCs were treated with demecolcine solution ( $380 \mathrm{ng} \mathrm{mL}^{-1}$; Sigma-Aldrich) for $5 \mathrm{~h}$ at $37^{\circ} \mathrm{C}$. Cells were detached with accutase (Thermo Fisher) for $1 \mathrm{~min}$ at $37^{\circ} \mathrm{C}$ collected in a $15 \mathrm{~mL}$ tube and centrifuged at $200 \mathrm{~g}, 5 \mathrm{~min}$ (RT). The supernatant was discarded, and the cell pellet resuspended in $1 \mathrm{~mL}$ ESM by tapping the tube carefully. Prewarmed $\left(37^{\circ} \mathrm{C}\right)$ hypotonic $\mathrm{KCl} /$ sodium citrate solution $(1 / 1$, $0.075 \mathrm{M} / 3.8 \mathrm{mM}$ ) was added dropwise to the cell suspension while shaking the tube carefully (approx. $8 \mathrm{~mL}$ ). After $15 \mathrm{~min}$ of incubation at $37^{\circ} \mathrm{C}$, the cells were centrifuged $(1000 \mathrm{rpm}, 8 \mathrm{~min}(\mathrm{RT}))$ and the supernatant discarded. Precooled $\left(-20^{\circ} \mathrm{C}\right)$ fixative $(3: 1$ methanol/glacial acetic acid $v / v$ ) was then added dropwise to the cell suspension while shaking the tube carefully. The cells were incubated on ice for $10 \mathrm{~min}$ and then centrifuged (fixation and centrifugation steps were repeated three times). Fixed cells were dropped onto slides and baked at $60^{\circ} \mathrm{C}$ overnight. Dry samples were immersed in $0.15 \%$ trypsin/ $\mathrm{NaCl}$ (Biochrom) for 50-60 s and stained with $5 \%$ Giemsa (Merck). A total of 11 Gbanded metaphases were analyzed from the selected cell lines. Karyotypes of baboon iPSCs were arranged according to Moore et al. (1999) using the IKAROS imaging system (Metasystems).

\subsection{Sequence comparison}

The following sequences were used for the determination of the similarity between the marmoset and the baboon cDNA and protein, respectively: SOX2 (ENSCJAG00000008401/ENSPANG00000025489), OCT4A (ENSCJAG00000019789/ENSPANG00000007627), KLF4 (ENSCJAG00000016955/XM_003911399.2 and ENSPANG00000025718), LIN28 (ENSCJAG00000009796/ENSPANG00000009841), c-MYC (ENSCJAG00000012620/ENSPANG00000010418), and NANOG (ENSCJAG00000018999/ENSPANG00000009019).

\section{Discussion}

NHP iPSCs are of relevance for the preclinical evaluation of regenerative therapies (Phillips et al., 2014). This may particularly involve therapies of the central and peripheral nervous system, the eye, the heart, and the vascular system (Stevens et al., 2009; Grow et al., 2016a; Agrba et al., 2016; Längin et al., 2018; Shiba et al., 2016; Emborg et al., 2013; Wang et al., 2015; Chong and Murry, 2014). Marmosets and macaques (rhesus and cynomolgus monkeys) are the most frequently used NHP species in biomedical research, but they differ in some characteristics from humans, including body size (Behr, 2015). In terms of body size, immunological characteristics, and other features, the baboon can be an excellent alternative model to mimic human pathology. The similar anatomy, specifically organ size, and immunology of baboons are reflected in their crucial role in (xeno-) transplantation research (Bailey, 2009; Längin et al., 2018). Since different iPSC lines from the same species, even from the same animal, have different properties and may respond differently to external cues, it is expedient to have a representative number of baboon iPSC lines available (Ohi et al., 2011; CarcamoOrive et al., 2017). So far, there are only very few baboon iPSC lines, and it has been reported that the reprogramming of cells particularly from this species is challenging in comparison to humans, macaques, or mice (Navara et al., 2013, 2018). Here, we report the generation and initial characterization of five new baboon iPSC lines and developed a robust protocol that can be used to easily increase the number of available lines.

We demonstrate that feeder-free culture of the novel baboon iPSC lines is possible, at least after initial derivation of the lines on MEFs. Although we tried to adapt all lines to feeder-free culture, this was not achieved for biPSC2 and 3 . We believe that further fine-tuning of the feeder-free culture conditions is necessary to generate a robust protocol that works for all lines. Feeder-free culture is important concerning preclinical testing, upscaling, and the molecular analy- 
sis of pure cell populations which are not intermingled by xeno-genic cells. Furthermore, the feeder-free protocol may pave the way to fully xeno-free generation of NHP iPSC lines (Villa-Diaz et al., 2013; Nishizawa et al., 2014; Hakala et al., 2009). Finally, in the context of the three Rs (replace, reduce, refine) of animal research, a reduction in the number of animals used needs to be considered since MEFs are prepared from E12.5 mouse embryos.

In the present study, we successfully generated iPSC lines from an adult female baboon. A previous report on baboon iPSCs used embryonic fibroblasts, which are much more easily reprogrammable than adult fibroblasts according to our own unpublished and previously published data (Debowski et al., 2015; Imamura et al., 2012). Generally, we have shown that our approach using the piggyBac system is robust and reproducible. Furthermore, we speculate that the efficiency of reprogramming of neonatal or even embryonic fibroblasts, using the same protocol, will be higher than using adult fibroblasts. However, this was not possible to test in our study due to the lack of tissue samples from young or even prenatal baboons.

The piggyBac cassette can be removed from the cells without leaving a footprint in the genome. This has been shown in previous reports with human and mouse iPSCs (Malik and Mahendra, 2013; Patel and Yang, 2010; Woltjen et al., 2011; Yusa et al., 2009; Mohseni et al., 2009) and could also be done with the Baboon lines. The option to remove the reprogramming cassette is a clear advantage of this system in comparison to retroviral vectors, which are stably integrated into the genome (Navara et al., 2013). Moreover, even if retroviral vectors are flanked by recombination sites, e.g., loxP sites, facilitating their excision, the excision site remains mutated due to incomplete removal of the recombination site. Currently, we cannot exclude that pluripotency factor expression from the transposon also supports feeder-free culture. After future removal of the transposon, the continuous culture on Geltrex needs to be reevaluated.

Protein expression analysis of the pluripotency factors in our biPSCs lines shows that they express OCT4A, LIN28, NANOG, SOX2 and SALL4, and tumor rejection antigens (Fig. 2). However, transcript analysis by RT-PCR, which can - in contrast to antibodies - discriminate between endogenous and exogenous factors, showed two things: first, the endogenous genes encoding the pluripotency factors are all reactivated, though at different levels (Fig. 3). Secondly, the piggyBac-derived transcripts are still detectable and therefore will contribute to the signals detected on the protein level by immunostaining (Fig. 2). In parallel studies with marmoset monkey iPSCs generated using the same system, we observed a silencing of the transposon and that the contribution of the exogenous expression to the overall pluripotency factor expression was (at least on the transcript level) most likely lower than the expression from the endogenous reactivation of pluripotency genes (Debowski et al., 2015). We speculate that passage-number- dependent promoter methylation may influence the expression level of the piggyBac cassette. In fact, we have evidence from piggyBac-mediated reprogramming-derived rhesus iPSC lines that methylation of the CAG promoter driving the reprogramming cassette occurs (unpublished data). Although the exogenous transposon-encoded factors are still active in cultured cells, the teratoma assays show that the iPSC lines are not arrested in pluripotency by the forced expression of the reprogramming factors. In fact, the teratomas show that expression from the piggyBac cassette is strongly downregulated, or even switched off, since in almost all cells of the teratoma (except the few clusters of cells like the one shown in Fig. S2) we were not able to detect OCT4A and other cassette-encoded factors on the protein level by robust and sensitive immunohistochemistry (Aeckerle et al., 2015; Wolff et al., 2019).

Altogether, as demonstrated by five novel iPSCs lines from an adult female olive baboon, we have established a robust protocol for the generation of adult baboon iPSC lines. In general, the fully reversible six-factor piggyBac system with monkey reprogramming factors seems to be an efficient tool for the generation of NHP iPSCs in general since we have successfully used the piggyBac system also for the generation of difficult-to-reprogram marmoset (Debowski et al. 2015) as well as of rhesus cells (unpublished). In cases where non-integrating approaches like the Sendai virus method do not work reliably, the piggyBac system represents an efficient alternative.

Data availability. Data sharing is generally not applicable to this article as this study analyses mostly qualitative data and no large datasets were generated during this study. Original data are available upon request.

Supplement. The supplement related to this article is available online at: https://doi.org/10.5194/pb-6-75-2019-supplement.

Author contributions. IRP and RB conceived and designed the experiments. IRP, MS, $\mathrm{AB}$, and RD performed the experiments. IRP, IB, and RB analyzed the data. RD and IB designed and performed the teratoma assay and karyotyping, respectively. RB and IRP wrote the paper with contributions from all coauthors. All authors approved the final version of the paper.

Competing interests. The authors declare that they have no conflict of interest.

Acknowledgements. We thank Nicole Umland, Angelina Berenson, Ulrike Goedecke, Anna Magerhans, Ilona Eggert, and Silke Günther for the excellent technical assistance and support dur- 
ing the experiments, Kerstin Mätz-Rensing for providing the baboon skin sample, and Kerstin Zaft for the administrative support.

Financial support. This research has been supported by the DZHK (grant no. 81Z0300201).

Review statement. This paper was edited by Eberhard Fuchs and reviewed by Hannes Klump, Julien Vezoli, and one anonymous referee

\section{References}

Aeckerle, N., Drummer, C., Debowski, K., Viebahn, C., and Behr, R.: Primordial germ cell development in the marmoset monkey as revealed by pluripotency factor expression: suggestion of a novel model of embryonic germ cell translocation, Mol. Hum. Reprod., 21, 552-552, https://doi.org/10.1093/molehr/gav016, 2015.

Agrba, V. Z., Porkhanov, V. A., Karal-Ogly, D. D., Leontyuk, A. V, Kovalenko, A. L., Sholin, I. Y., Gvozdik, T. E., Ignatova, I. E., Agumava, A. A., Chuguev, Y. P., Gvaramiya, I. A., and Lapin, B. A.: Transplantation of Simian Mesenchymal Stem Cells to Baboons with Experimentally Induced Myocardial Infarction, B. Exp. Biol. Med., 160, 589-591, https://doi.org/10.1007/s10517016-3223-7, 2016.

Bailey, L.: The Baboon in Biomedical Research - The Baboon in Xenotransplant Research, Springer, edited by: WilliamsBlangero, S., Tardif, S. D., and VandeBerg, J. L., Springer, New York, NY, 2009.

Behr, R.: Primate biologics research at a crossroads, Potential of Genetically Modified Nonhuman Primate Models for Biomedicine, edited by: Weinbauer, G. F. and Vogel, F., Waxmann, Münster, New York, 2015.

Carcamo-Orive, I., Hoffman, G. E., Cundiff, P., Beckmann, N. D., D'Souza, S. L., Knowles, J. W., Patel, A., Papatsenko, D., Abbasi, F., Reaven, G. M., Whalen, S., Lee, P., Shahbazi, M., Henrion, M. Y. R., Zhu, K., Wang, S., Roussos, P., Schadt, E. E., Pandey, G., Chang, R., Quertermous, T., and Lemischka, I.: Analysis of Transcriptional Variability in a Large Human iPSC Library Reveals Genetic and Non-genetic Determinants of Heterogeneity, Cell Stem Cell, 20, 1-15, https://doi.org/10.1016/j.stem.2016.11.005, 2017.

Chong, J. J. H. and Murry, C. E.: Cardiac Regeneration Using Pluripotent Stem Cells - Progression to Large Animal Models, Stem Cell Res., 13, 654-665, https://doi.org/10.1016/j.scr.2014.06.005, 2014.

Cox, L. A., Comuzzie, A. G., Havill, L. M., Karere, G. M., Spradling, K. D., Mahaney, M. C., Nathanielsz, P. W., Nicolella, D. P., Shade, R. E., Voruganti, S., and VandeBerg, J. L.: Baboons as a model to study genetics and epigenetics of human disease, ILAR J., 54, 106-121, https://doi.org/10.1093/ilar/ilt038, 2013.

Debowski, K., Warthemann, R., Lentes, J., Salinas-Riester, G., Dressel, R., Langenstroth, D., Gromoll, J., Sasaki, E., and Behr, R.: Non-viral generation of marmoset monkey iPS cells by a six-factor-in-one-vector approach, PLoS One, 10, 1-21, https://doi.org/10.1371/journal.pone.0118424, 2015.
Didier, E. S., MacLean, A. G., Mohan, M., Didier, P. J., Lackner, A. A., and Kuroda, M. J.: Contributions of Nonhuman Primates to Research on Aging, Vet. Pathol., 53, 277-290, https://doi.org/10.1177/0300985815622974, 2016.

Eildermann, K., Aeckerle, N., Debowski, K., Godmann, M., Christiansen, H., Heistermann, M., Schweyer, S., Bergmann, M., Kliesch, S., Gromoll, J., Ehmcke, J., Schlatt, S., and Behr, R.: Developmental expression of the pluripotency factor sal-like protein 4 in the monkey, human and mouse testis: Restriction to premeiotic germ cells, Cells Tissues Organs, 196, 206-220, https://doi.org/10.1159/000335031, 2012.

Emborg, M. E., Liu, Y., Xi, J., Zhang, X., Yin, Y., Lu, J., Joers, V., Swanson, C., Holden, J. E., and Zhang, S.: Induced Pluripotent Stem Cell-Derived Neural Cells Survive and Mature in the Nonhuman Primate Brain, Cell Rep., 3, 646-650, https://doi.org/10.1016/j.celrep.2013.02.016, 2013.

Grow, D. A., McCarrey, J. R., and Navara, C. S.: Advantages of nonhuman primates as preclinical models for evaluating stem cell-based therapies for Parkinson's disease, Stem Cell Res., 17, 352-366, https://doi.org/10.1016/j.scr.2016.08.013, 2016a.

Grow, D. A., Simmons, D. V., Gomez, J. A., Wanat, M. J., McCarrey, J. R., Paladini, C. A., and Navara, C. S.: Differentiation and Characterization of Dopaminergic Neurons From Baboon Induced Pluripotent Stem Cells, Stem Cells Transl. Med., 5, 11331144, https://doi.org/10.5966/sctm.2015-0073, 2016 b.

Hakala, H., Rajala, K., Ojala, M., Panula, S., Areva, S., Kellomäki, M., Suuronen R., and Skottman H.: Comparison of Biomaterials and Extracellular Matrices as a Culture Platform for Multiple, Independently Derived Human Embryonic Stem Cell Lines, Tissue Eng., 15, 1775-1785, https://doi.org/10.1089/ten.tea.2008.0316, 2009.

Imamura, M., Okuno, H., Tomioka, I., Kawamura, Y., Lin, Z. Y.-C., Nakajima, R., Akamatsu, W., Okano, H. J., Matsuzaki, Y., Sasaki, E., and Okano, H.: Derivation of Induced Pluripotent Stem Cells by Retroviral Gene Transduction in Mammalian Species, Methods Mol. Biol., 925, 277-294, https://doi.org/10.1007/978-1-62703-011-3, 2012.

Kim, K.-Y., Hysolli, E., and Park, I.-H.: Reprogramming Human Somatic Cells into Induced Pluripotent Stem Cells (iPSCs) Using Retroviral Vector with GFP, J. Vis. Exp., 62, 2-5, https://doi.org/10.3791/3804, 2012.

Kimbrel, E. A. and Lanza, R.: Current status of pluripotent stem cells: Moving the first therapies to the clinic, Nat. Rev. Drug Discov., 14, 681-692, https://doi.org/10.1038/nrd4738, 2015.

Kobayashi, Y., Okada, Y., Itakura, G., Iwai, H., Nishimura, S., Yasuda, A., Nori, S., Hikishima, K., Konomi, T., Fujiyoshi, K. Tsuji, O., Toyama, Y., Yamanaka, S., Nakamura, M., and Okano, H.: Pre-Evaluated Safe Human iPSC-Derived Neural Stem Cells Promote Functional Recovery after Spinal Cord Injury in Common Marmoset without Tumorigenicity, PLoS One, 7, 1-13, https://doi.org/10.1371/journal.pone.0052787, 2012.

Längin, M., Mayr, T., Reichart, B., Michel, S., Buchholz, S., Guethoff, S., Dashkevich, A., Baehr, A., Egerer, S., Bauer, A., Mihalj, M., Panelli, A., Issl, L., Ying, J., Fresch, A. K., Buttgereit, I., Mokelke, M., Radan, J., Werner, F., Lutzmann, I., Steen, S., Sjöberg, T., Paskevicius, A., Qiuming, L., Sfriso, R., Rieben, R., Dahlhoff, M., Kessler, B., Kemter, E., Klett, K., Hinkel, R., Kupatt, C., Falkenau, A., Reu, S., Ellgass, R., Herzog, R., Binder, U., Wich, G., Skerra, A., Ayares, D., Kind, 
A., Schönmann, U., Kaup, F.-J., Hagl, C., Wolf, E., Klymiuk, N., Brenner, P., and Abicht, J.-M.: Consistent success in lifesupporting porcine cardiac xenotransplantation, Nature, 564. 430-433, https://doi.org/10.1038/s41586-018-0765-z, 2018.

Malik, N. and Mahendra, S. R.: A Review of the Methods for Human iPSC Derivation, Methods Mol Biol., 997, 23-33, https://doi.org/10.1007/978-1-62703-348-0, 2013.

Mohseni, P., Woltjen, K., Kaji, K., Paca, A., Mileikovsky, M., and Norrby, K.: Virus-free induction of pluripotency and subsequent excision of reprogramming factors, Nature, 458, 771-775, https://doi.org/10.1038/nature07864, 2009.

Moore, C. M., Janish, C., Eddy, C. A., Hubbard, G. B., Leland, M. M., and Rogers, J.: Cytogenetic and Fertility Studies of a Rheboon, Rhesus Macaque (Macaca mulatta) Baboon (Papio hamadryas) Cross: Further Support for a Single Karyotype Nomenclature, Am. J. Phys. Anthropol., 127, 119-127, https://doi.org/10.1002/(SICI) 10968644(199910)110:2<119::AID-AJPA1>3.0.CO;2-S, 1999.

Navara, C. S., Hornecker, J., Grow, D., Chaudhari, S., Hornsby, P. J., Ichida, J. K., Eggan, K., and McCarrey, J. R.: Derivation of induced pluripotent stem cells from the baboon: a nonhuman primate model for preclinical testing of stem cell therapies, Cell. Reprogram., 15, 495-502, https://doi.org/10.1089/cell.2012.0093, 2013.

Navara, C. S., Chaudhari, S., and McCarrey, J. R.: Optimization of culture conditions for the derivation and propagation of baboon (Papio anubis) induced pluripotent stem cells, PLoS One, 13, 1 16, https://doi.org/10.1371/journal.pone.0193195, 2018.

Nishizawa, M., Yamanaka, S., Ichisaka, T., Takizawa, N., Taniguchi, Y., Toyoda, T., Osafune, K., Takahashi, J., Sekiguchi, K., Nakagawa, M., Doi, D., Senda, S., Asano, K., Yoshida, Y., and Morizane, A.: A novel efficient feeder-free culture system for the derivation of human induced pluripotent stem cells, Sci. Rep., 4, 1-7, https://doi.org/10.1038/srep03594, 2014.

Nishizawa, M., Chonabayashi, K., Nomura, M., Tanaka, A., Nakamura, M., Inagaki, A., Nishikawa, M., Takei, I., Oishi, A., Tanabe, K., Ohnuki, M., Yokota, H., Koyanagi-Aoi, M., Okita, K., Watanabe, A., Takaori-Kondo, A., Yamanaka, S., and Yoshida, Y.: Epigenetic Variation between $\mathrm{Hu}-$ man Induced Pluripotent Stem Cell Lines Is an Indicator of Differentiation Capacity, Cell Stem Cell, 19, 341-354, https://doi.org/10.1016/j.stem.2016.06.019, 2016.

Ohi, Y., Qin, H., Hong, C., Blouin, L., Polo, J. M., Guo, T., Qi, Z., Downey, S. L., Manos, P. D., Rossi, D. J., Yu, J., Hebrok, M., Hochedlinger, K., Costello, J. F., and Song, J. S.: Incomplete DNA methylation underlies a transcriptional memory of the somatic cell in human iPS cells, Nat Cell Biol., 13, 541-549, https://doi.org/10.1038/ncb2239, 2011.

Patel, M. and Yang, S.: Advances in Reprogramming Somatic Cells to Induced Pluripotent Stem Cells, Stem Cell Rev., 6, 367-380, https://doi.org/10.1007/s12015-010-9123-8, 2010.

Phillips, K. A., Bales, K. L., Capitanio, J. P., Conley, A., Czoty, P. W., 't Hart, B. A., Hopkins, W. D., Hu, S. L., Miller, L. A. Nader, M. A., Nathanielsz, P. W., Rogers, J., Shively, C. A., and Voytko, M. Lou: Why primate models matter, Am. J. Primatol., 76, 801-827, https://doi.org/10.1002/ajp.22281, 2014.

Rogers, J. and Hixson, J. E.: Baboons as an Animal Model for Genetic Studies of Common Human Disease, Am. J. Hum. Genet., 61, 489-493, 1997.
Shearer, M. H., Dark, R. D., Chodosh, J., and Kennedy, R. C.: Comparison and Characterization of Immunoglobulin G Subclasses among Primate Species Comparison and Characterization of Immunoglobulin G Subclasses among Primate Species, Clin. Diagn. Lab. Immun., 6, 953-958, 1999.

Shiba, Y., Gomibuchi, T., Seto, T., Wada, Y., Ichimura, H., Tanaka, Y., Ogasawara, T., Okada, K., Shiba, N., Sakamoto, K., Ido, D., Shiina, T., Ohkura, M., Nakai, J., Uno, N., Kazuki, Y., Oshimura, M., Minami, I., and Ikeda, U.: Allogeneic transplantation of iPS cell-derived cardiomyocytes regenerates primate hearts, Nature, 538, 388-391, https://doi.org/10.1038/nature19815, 2016.

Simerly, C. R., S.Navara, C., Castro, C. A., Turpin, J. C., Redinger, C. J., JocelynD.Mich-Basso, Jacoby, E. S., Grund, K. J., McFarland, D. A., Oliver, S. L., Ben-Yehudah, A., Carlisle, D. L., Frost, P., Penedo, C., Hewitson, L., and Schatten, G.: Establishment and characterization of baboon embryonic stem cell lines: An Old World Primate model for regeneration and transplantation research, Stem Cell Res., 2, 178-187, https://doi.org/10.1016/j.scr.2009.02.004, 2009.

Sosa, E., Kim, R., Rojas, E. J., Hosohama, L., Hennebold, J. D., Orwig, K. E., and Clark, A. T.: An integration-free, virus-free rhesus macaque induced pluripotent stem cell line (riPSC89) from embryonic fibroblasts, Stem Cell Res., 21, 5-8, https://doi.org/10.1016/j.scr.2017.03.011, 2017.

Stevens, K. R., Bendixen, K., Regnier, M., Dupras, S. K., Muskheli, V., Kreutziger, K. L., Reinecke, H., Nourse, M. B., Korte, F. S., and Murry, C. E.: Physiological function and transplantation of scaffold-free and vascularized human cardiac muscle tissue, P. Natl. Acad. Sci. USA, 106, 16568-16573, https://doi.org/10.1073/pnas.0908381106, 2009.

Takahashi, K. and Yamanaka, S.: Induction of Pluripotent Stem Cells from Mouse Embryonic and Adult Fibroblast Cultures by Defined Factors, Cell, 126, 663-676, https://doi.org/10.1016/j.cell.2006.07.024, 2006.

Takahashi, K., Tanabe, K., Ohnuki, M., Narita, M., Ichisaka, T., and Tomoda, K.: Induction of Pluripotent Stem Cells from Adult Human Fibroblasts by Defined Factors, Cell, 131, 861-872, https://doi.org/10.1016/j.cell.2007.11.019, 2007.

Thomson, J. A., Kalishman, J., Golos, T. G., Durning, M., Harris, C. P., Becker, R. A., and Hearn, J. P.: Isolation of a primate embryonic stem cell line, P. Natl. Acad. Sci. USA, 92, 7844 7848,https://doi.org/10.1073/pnas.92.17.7844, 1995.

Tiburcy, M., Hudson, J. E., Balfanz, P., Schlick, S., Meyer, T., Liao, M. L. C., Levent, E., Raad, F., Zeidler, S., Wingender, E., Riegler, J., Wang, M., Gold, J. D., Kehat, I., Wettwer, E., Ravens, U., Dierickx, P., Van Laake, L. W., Goumans, M. J., Khadjeh, S., Toischer, K., Hasenfuss, G., Couture, L. A., Unger, A., Linke, W. A., Araki, T., Neel, B., Keller, G., Gepstein, L., Wu, J. C., and Zimmermann, W. H.: Defined engineered human myocardium with advanced maturation for applications in heart failure modeling and repair, Circulation, 135, 1832-1847, https://doi.org/10.1161/CIRCULATIONAHA.116.024145, 2017.

Turnbull, I. C., Karakikes, I., Serrao, G. W., Backeris, P., Lee, J. J., Xie, C., Senyei, G., Gordon, R. E., Li, R. A., Akar, F. G., Hajjar, R. J., Hulot, J. S., and Costa, K. D.: Advancing functional engineered cardiac tissues toward a preclinical model of human myocardium, FASEB J., 28, 644-654, https://doi.org/10.1096/fj.13228007, 2014. 
Varilly, P. and Chandler, D.: An age-old paradigm challenged Old baboons generate vigorous humoral immune responses to LcrV, a plague antigen, J. Immunol., 181, 109-115, https://doi.org/10.4049/jimmunol.181.1.109, 2008.

Villa-Diaz, L. G., Ross, A. M., Lahann, J., and Krebsbach, P. H.: Concise review: The evolution of human pluripotent stem cell culture: From feeder cells to synthetic coatings, Stem Cells, 31, 1-7, https://doi.org/10.1002/stem.1260, 2013.

Wang, S., Zou, C., Fu, L., Wang, B., An, J., Song, G., Wu, J., Tang, X., Li, M., Zhang, J., Yue, F., Zheng, C., Chan, P., Zhang, Y. A., and Chen, Z.: Autologous iPSC-derived dopamine neuron transplantation in a nonhuman primate Parkinson's disease model, Cell Discov., 1, 1-11, https://doi.org/10.1038/celldisc.2015.12, 2015.

Wolff, E., Suplicki, M. M., and Behr, R.: Primordial germ cells do not migrate along nerve fibres in marmoset monkey and mouse embryos, Reproduction, 157, 101-109, https://doi.org/10.1530/REP-18-0401, 2019.

Woltjen, K., Hämäläinen, R., Mark Kibschull, Mileikovsky, M., an Nagy, A.: Transgene-free production of pluripotent stem cells using piggyBac transposons, Methods Mol. Biol., 767, 87-103, https://doi.org/10.1007/978-1-61779-201-4_7, 2011.
Yamanaka, S., Okita, K., Sato, Y., Saji, H., Okamoto, S., Takahashi, M., Tanabe, K., Takahashi, J., Tezuka, K., Shibata, T., Hong, H., Matsumura, Y., Kunisada, T., Nakagawa, M., Morizane, A., and Okada, A.: A more efficient method to generate integration-free human iPS cells, Nat. Methods, 8, 409-412, https://doi.org/10.1038/nmeth.1591, 2011.

Yu, J., Hu, K., Smuga-otto, K., Tian, S., Stewart, R., Igor, I., and Thomson, J. A.: Human Induced Pluripotent Stem Cells Free of Vector and Transgene Sequences, Science, 324, 797-801, https://doi.org/10.1126/science.1172482, 2009.

Yusa, K., Rad, R., Takeda, J., and Bradley, A.: Generation of transgene-free induced pluripotent mouse stem cells by the piggyBac transposon, Nat. Methods, 6, 363-369, https://doi.org/10.1038/nmeth.1323, 2009.

Zhang, X., Cao, H., Bai, S., Huo, W., and Ma, Y.: Differentiation and characterization of rhesus monkey atrial and ventricular cardiomyocytes from induced pluripotent stem cells, Stem Cell Res., 20, 21-29, https://doi.org/10.1016/j.scr.2017.02.002, 2017. 
Supplement of Primate Biol., 6, 75-86, 2019 https://doi.org/10.5194/pb-6-75-2019-supplement (c) Author(s) 2019. This work is distributed under the Creative Commons Attribution 4.0 License.

Supplement of

Baboon induced pluripotent stem cell generation by piggyBac transposition of reprogramming factors

Ignacio Rodriguez-Polo et al.

Correspondence to: Rüdiger Behr (rbehr@dpz.eu)

The copyright of individual parts of the supplement might differ from the CC BY 4.0 License. 

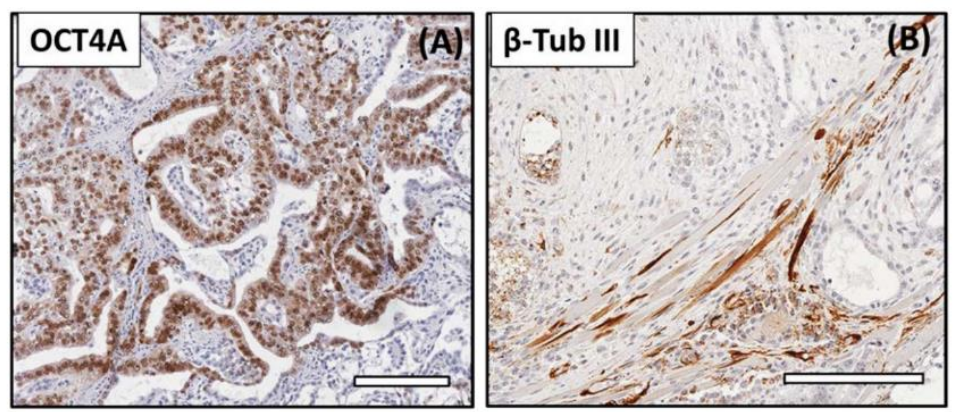

Suppl. Figure 1: Immunofluorescence isotype control (A), (B), (C) Isotype controls, merge images FITC+DAPI (scale bar 20 $\mu \mathrm{m}$ ) (A) Isotype control Mouse IgM (B) Isotype control Mouse IgG (C) Isotype control Rabbit IgG.
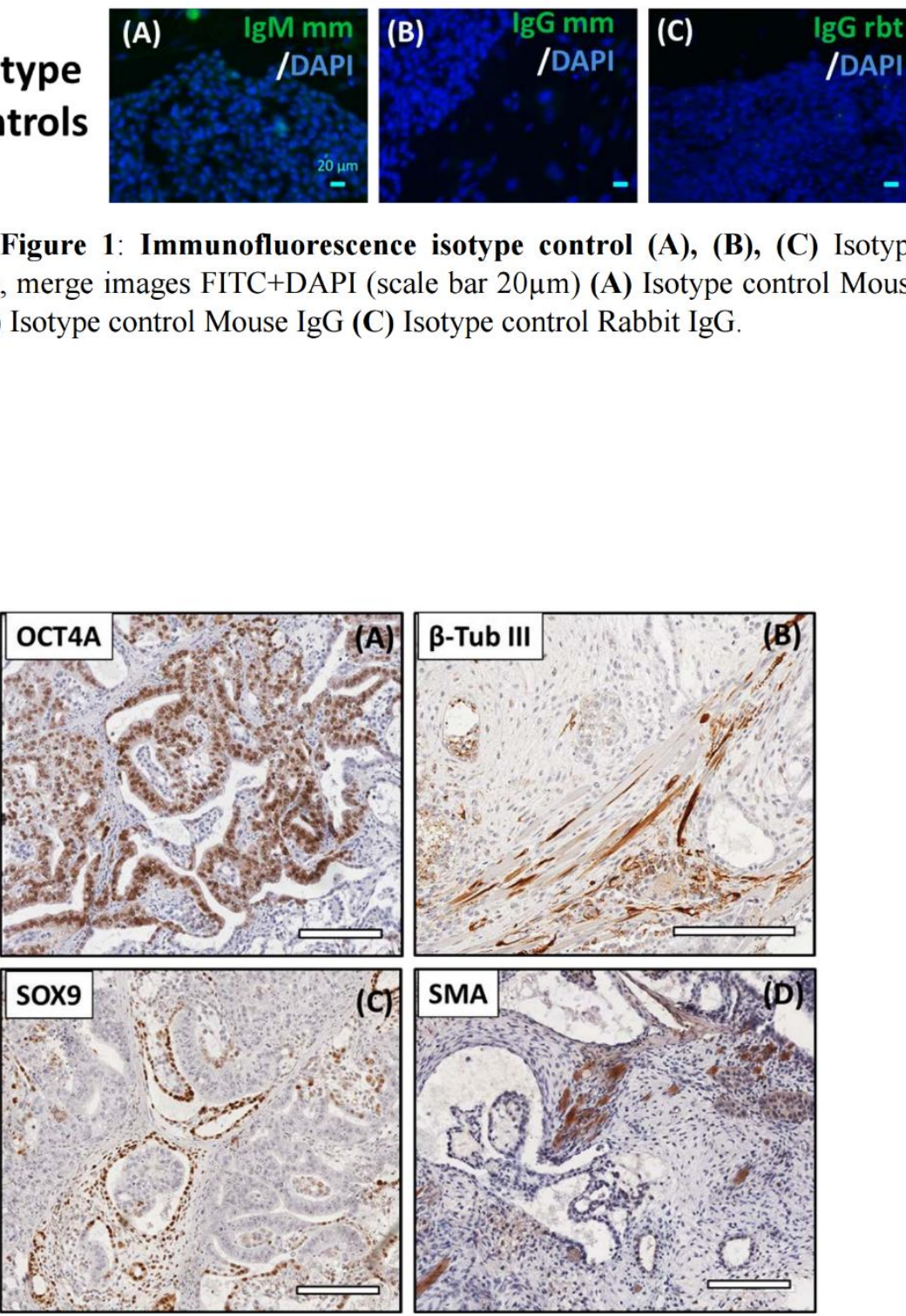

Suppl. Figure 2: Immunohistochemical analysis of DPZ_biPSC 5 teratoma. Tumor tissue analyzed for the expression of SOX9, $\beta$-Tubulin III, and smooth muscle actin (SMA), and OCT4A. (A) Pluripotency marker OCT4A (POU5F1) staining (bars represent $200 \mu \mathrm{m}$ ). (B) Ectodermal $\beta$-Tubulin 3 staining ( $\beta$-Tub III). (C) SOX9 staining suggesting endodermal lineage differentiation. (D) Mesodermal smooth muscle actin (SMA) staining. 


\section{Chapter 2: Reproducible Primate iPSC Generation, Cultivation, and Cardiac Differentiation under Chemically Defined Conditions}

\section{Author contribution statement}

MS, IRP, and RB conceived and designed the experiments. MS, IRP, WH, TB, MT, and DYK performed the experiments. MS, IRP, IB, WH, DYK, MT, KS, WZ and RB analyzed the data. RD and IB designed and performed the teratoma assay and karyotyping, respectively. MS, IRP, TB, RB, and KS performed the MEA measurements and analysis. MT and WZ performed the EHM experiments and analyzed the data. RB and MS wrote the paper with contributions from all coauthors.

\begin{tabular}{|c|c|}
\hline \multicolumn{2}{|r|}{ Author contribution statement } \\
\hline \multicolumn{2}{|r|}{ Chapter 2: Reproducible Primate iPSC Generation, Cultivation, and } \\
\hline \multicolumn{2}{|r|}{ Cardiac Differentiation under Chemically Defined Conditions } \\
\hline \multirow{3}{*}{ Figure 1} & IRP and DYK during her Master thesis (under the supervision \\
\hline & of IRP and MS) performed the reprogramming efficiency \\
\hline & analysis \\
\hline \multirow{2}{*}{ Figure 2} & MS and WH during his Master thesis (under the supervision \\
\hline & of MS) empirically developed the UPPS medium \\
\hline \multirow{2}{*}{ Figure 3, Suppl. Figure 2} & MS, IRP, DYK, and WH performed the characterization of the \\
\hline & generated lines \\
\hline Figure 3 & MS, IRP, DYK, and WH prepareed the karyotypes, IB stained and analyzed the karyograms \\
\hline Figure 4 & MS and DYK performed EB formation assay \\
\hline \multirow{2}{*}{ Figure 4} & $\mathrm{RD}$ performed the teratoma formation assay \\
\hline & MS, RB, and RD analyzed the teratoma histology \\
\hline \multirow{2}{*}{ Figure 5} & MS, WH, and IRP tested different cardiomyocyte \\
\hline & differentiation protocols until finding the appropriate one for NHP \\
\hline Figure 5 & DYK evaluate the differentiation efficiencies (under the supervision of IRP and MS) \\
\hline \multirow{2}{*}{ Figure 5} & IRP, MS, and TB MEA performed measurements \\
\hline & IRP, TB, and KSB performed MEA data analysis \\
\hline Figure 6 & EHM experiments and analysis were performed by MT and WZ \\
\hline Suppl. Figure 1 & MS adapted the images of the phylogenetic trees \\
\hline Table 4 & DYK performed the similarity analysis \\
\hline Experiment design & MS, IRP, and RB \\
\hline Manuscript writing & MS and RB, with the contribution from all authors \\
\hline
\end{tabular}




\title{
Non-Human Primate iPSC Generation, Cultivation, and Cardiac Differentiation under Chemically Defined Conditions
}

\begin{abstract}
Michael Stauske ${ }^{1,2,3}$, Ignacio Rodriguez Polo ${ }^{1,2}$, Wadim Haas ${ }^{1}$, Debbra Yasemin Knorr $^{1,4}$, Thomas Borchert ${ }^{2,5}$, Katrin Streckfuss-Bömeke ${ }^{2,5}$, Ralf Dressel ${ }^{2,6}$, Iris Bartels $^{7}$, Malte Tiburcy ${ }^{2,8}$, Wolfram-Hubertus Zimmermann ${ }^{2,8}$, Rüdiger Behr ${ }^{1,2 *}$
\end{abstract}

${ }^{1}$ Research Platform Degenerative Diseases, German Primate Center - Leibniz Institute for Primate Research, Kellnerweg 4, 37077 Göttingen, Germany

${ }^{2}$ DZHK (German Center for Cardiovascular Research), partner site Göttingen, Germany

${ }^{3}$ Current address: BlueRock Therapeutics, 101 College St, PMCRT 14-301, Toronto, ON, M5G 1L7, Canada

${ }^{4}$ Current address: Johann-Friedrich-Blumenbach Institute for zoology and anthropology; Department of Cellular Neurobiology, Julia-Lermontowa-Weg 3, 37075 Göttingen, Germany

${ }^{5}$ Department of Cardiology and Pneumology, University Medical Center Göttingen, Robert-Koch-Str. 40, 37075 Göttingen, Germany

${ }^{6}$ Institute of Cellular and Molecular Immunology, University Medical Center Göttingen, Robert-KochStr. 40, 37075 Göttingen, Germany

${ }^{7}$ Institute of Human Genetics, University Medical Center Göttingen, Robert-Koch-Str. 40, 37075 Göttingen, Germany

${ }^{8}$ Institute of Pharmacology and Toxicology, University Medical Center Göttingen, Robert-Koch-Str. 40, 37075 Göttingen, Germany

*Correspondence: $\quad$ Rüdiger Behr

Research Platform Degenerative Diseases

German Primate Center - Leibniz Institute for Primate Research

Kellnerweg 4, 37077 Göttingen, Germany

Fax: 0049-(0)551-3851403

Email: rbehr@dpz.eu

\begin{abstract}
Non-human primates (NHP) are essential for preclinical testing of advanced therapy medicinal products (ATMPs), including induced pluripotent stem cell (iPSC)based therapies. However, testing and validation of ATMPs is hampered by a variety of experimental procedures. Studies published on heart and brain regeneration in NHP differ in culture conditions and stem cell source. To the present, the number of iPSClines tested within these studies is low and yet relies on xenogenic support. The resulting line-dependant variability poses a risk for future clinical application of the
\end{abstract}




\section{Chapter 2: Reproducible Primate iPSC Generation, Cultivation, and Cardiac Differentiation under Chemically Defined Conditions}

NHP-iPSC. Here, we demonstrate that commercial "human" PSC media are not sufficient for undifferentiated NHP-PSC culture. Additionally, rhesus fibroblast reprogramming is less efficient compared to human cells. We established a robust and universal protocol for transgene- and feeder-free primate iPSC generation and longterm cultivation. Directed differentiation of the iPSCs into beating cardiomyocytes and engineered heart muscle demonstrated their value for preclinical ATMP testing. In summary, we provide a standardized protocol for the generation of genetically nonmodified primate iPSCs under chemically defined conditions.

\section{Introduction}

The value of non-human primates (NHP) as animal models in translational research is increasingly recognized ${ }^{1}$. NHP offer advantages compared to other species because of their close phylogenetic relationship to humans, reflected in similarities in anatomy, a primate-specific genetic constitution and pathology ${ }^{2-7}$. This makes NHP predictive models for the preclinical testing of new treatments for human diseases. In fact, NHP are not only used in classical toxicology studies 8910 , but they are also essential in basic research ${ }^{11} 12$ and for the testing of advanced therapy medicinal products (ATMPs) including pluripotent stem cells (PSCs) ${ }^{13}$. Since it is possible to generate genomically non-modified human induced pluripotent stem cells (iPSCs) ${ }^{14} 151617$, these cells are moving forward towards clinical application ${ }^{181920}$. Consequently, several studies used NHP models for preclinical PSC-based transplantation studies in the fields of cardiology and neurodegenerative diseases, amongst others ${ }^{20-26}$.

Studies on NHP-PSC-based cell replacement therapies differ in the experimental setup and species origin of stem cells tested. While Kikuchi et al., ${ }^{24}$ and Chong and collaborators ${ }^{21}$ tested the regeneration potential of human iPSC in monkeys, Shiba et al., ${ }^{20}$ and Wang et al., ${ }^{26}$ focused on allogenic and autologous iPSC transplantation. Moreover, numerous different protocols have been published for the generation and cultivation of NHP-iPSCs. To our knowledge, they all relied on feeder cells and often xenogenic serum for iPSC cultivation ${ }^{27}$. Moreover, most iPSC lines were generated using integrating retroviral vectors ${ }^{26} 28$. Furthermore, limited numbers of PSC lines, often only one, were generated and tested disregarding inter-iPSC line variability 2026 . One reason for this could be the lower reprogramming efficiencies of NHP compared to human cells. For instance, marmoset monkey iPSCs were initially obtained only from highly proliferative neonatal cells with five or even six reprogramming factors instead of the classical four Yamanaka factors ${ }^{29}{ }^{30}$. Stabilization of a marmoset iPSC with the classical four Yamanaka factors from marmoset was successful only after $\sim 100$ days of reprogramming ${ }^{31}$. Furthermore, the generation of stable macaque iPSC lines is challenging and less efficient in comparison to human or mouse ${ }^{26}$. This is supported by own observations and personal communications with other laboratories. However, for preclinical development of live ATMPs it is critically important, to make procedures reproducible. To justify iPSC-based clinical trials the establishment of 


\section{Chapter 2: Reproducible Primate iPSC Generation, Cultivation, and Cardiac Differentiation under Chemically Defined Conditions}

standardized protocols is of utmost importance. Generation of these protocols suitable for both human and NHP will allow future clinical translation. Besides reproducibility, it is instructive to generate and maintain NHP- and human-iPSCs under the same conditions in order to test iPSC-based therapies pre-clinically in a meaningful way. Finally, highly standardized and reproducible procedures will allow full exploration of the differences between allogenic and autologous iPSC-derived ATMPs.

In this study, we developed a robust and reproducible protocol for transgene- and feeder-free human and NHP-iPSCs establishment. We included the rhesus macaque (Macaca mulatta) and the olive baboon (Papio anubis), as both NHP species are relevant in preclinical research ${ }^{1}$. We systematically developed and critically refined primate iPSC long-term culture. This protocol allows the undifferentiated "clinicalgrade cultivation" of rhesus, baboon and human PSCs under identical and chemically defined conditions. We further found that human cardiac differentiation protocols ${ }^{32} 33$ are ineffective with NHP-iPSCs. Therefore, we developed a novel time- and costoptimized protocol for the directed cardiac differentiation of NHP-iPSCs. This protocol leads to robust cardiomyocyte differentiation with high efficiencies of rhesus, baboon, and human iPSCs. The resulting spontaneously beating cells can be used for engineered heart muscle generation, responding to external calcium and $\beta$-adrenergic stimuli.

\section{Results}

\subsection{Rhesus monkey fibroblast reprogramming is less efficient than human fibroblast reprogramming}

We aimed to generate integration-free NHP- and human-iPSCs under feeder- and serum-free conditions using an easy and cost-effective reprogramming method. Therefore, we used established episomal vectors containing OCT4, SOX2, KLF4, LMYC, and LIN28 as well as an shRNA against $p 53^{15}$. For the study we selected two NHP species, rhesus macaque and baboon phylogenetically close to human and with biomedical relevance (Fig.S1). We consistently noted higher reprogramming efficiencies of human compared to NHP. To quantify our observations, we reprogrammed fibroblasts from three different adult rhesus macaques and two human adults under the same conditions. We counted the alkaline phosphatase (AP) expressing primary colonies at day 20,30 and 40 after nucleofection. Human primary colonies appeared earlier and at much higher numbers (Fig.1). Differences were particularly evident at day 20 with 10-50 times more human colonies compared to macaque (Fig.1B). In the following time points, the deviation became smaller due to the delayed appearance of rhesus cell colonies. Additionally, fusion of the expanding human cell colonies further resulted in a reduced number of distinguishable colonies. In summary, reprogramming of adult rhesus monkey fibroblasts is less efficiently 


\section{Chapter 2: Reproducible Primate iPSC Generation, Cultivation, and Cardiac Differentiation under Chemically Defined Conditions}

compared to adult human fibroblasts under identical conditions. However, feeder-free reprogramming of putative rhesus fibroblasts was successful and reproducible. Using this approach, we reprogrammed human, rhesus macaque, and baboon fibroblasts.

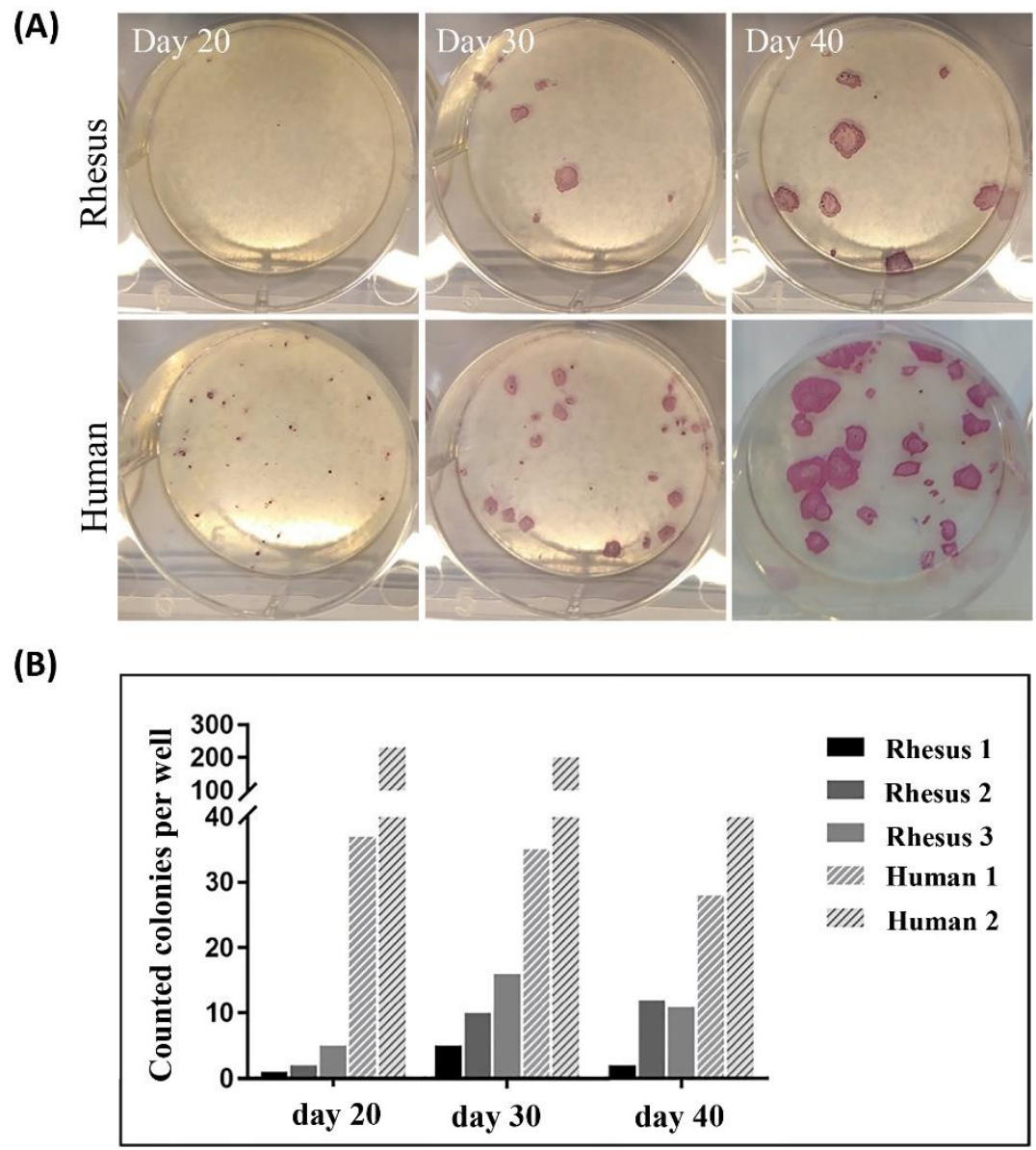

Figure 1. Comparison of reprogramming efficiencies between rhesus and human fibroblasts. (A) Skin fibroblasts from three different rhesus macaques and two human donors were nucleofected with episomal vectors containing human reprogramming genes. Appearing colonies were stained for alkaline phosphatase and counted at day 20,30 and 40 after nucleofection (exemplarily shown for one human and one rhesus reprogramming). (B) The colony count confirmed lower reprogramming efficiencies in the rhesus cells compared to the human cells (Colonies per well).

\subsection{Undifferentiated long-term culture of NHP-PSCs failed under "human" feeder-free conditions}

Two commercially available media, namely Essential 8 (E8) and StemMACS iPS Brew XF, were tested for long term cultivation of feeder-free iPSC. Rhesus ESC were cultured in parallel as positive control for the tested human, rhesus and baboon iPSC (line 366.4 ${ }^{34}$ Thomson et al., 1995). While human cells remained undifferentiated in both media, NHP cells failed to survive in either. E8 and StemMACS iPS Brew XF allowed the long-term undifferentiated culture of human PSCs (Fig.2 for E8 medium; data not shown for iPS Brew XF). However, long-term cell culture of newly generated 
NHP-iPSCs as well as rhesus macaque ESCs failed in both media. The NHP-PSCs showed clear signs of differentiation with loss of typical compact colony morphology when compared with undifferentiated human iPSCs (Fig.2). Additionally, the morphology of individual NHP cells changed over time, exhibiting a lower nucleus:cytoplasm ratio and increased cell size when compared with the human iPSCs (Fig.2, insets). Altogether, the morphological differences between the human and the NHP-PSCs in identical commercial media were evident, although their morphology was reported to be similar under conventional feeder-based culture conditions (Chapter 1) ${ }^{28}$. In conclusion, there are differences between human and NHP-PSCs regarding reprogramming efficiencies and their requirements for long-term undifferentiated culture under feeder- and serum-free conditions.

\subsection{Long-term cultivation of NHP- and human-iPSCs in the newly developed UPPS medium}

Based on the above findings, we tested media that allowed long-term cultivation of iPSCs. Both E8 and iPS Brew were supplemented with variable combinations of small molecules and BMP4. iPSC from all three species were maintained for at least 3 passages in the newly designed conditions (Suppl. Table 5). Finally, a combination of StemMACS iPS Brew XF medium supplemented with IWR-1 and Chir99021 was able to maintain both NHP and human PSCs in a morphologically undifferentiated state. During subsequent protocol optimization, we stepwise decreased the concentrations of IWR-1 and Chir99021 in order to determine the minimum concentration of these small molecules for the undifferentiated culture of NHP and human PSCs. Ultimately, the combination of Stem MACS iPS Brew XF medium supplemented with $1 \mu \mathrm{M}$ IWR-1 and $0.5 \mu \mathrm{M}$ Chir99021 stabilized the iPSC lines, keeping them in an undifferentiated state over long culture periods of more than 50 passages (Fig.2). We termed this medium universal primate pluripotent stem cell (UPPS) medium.

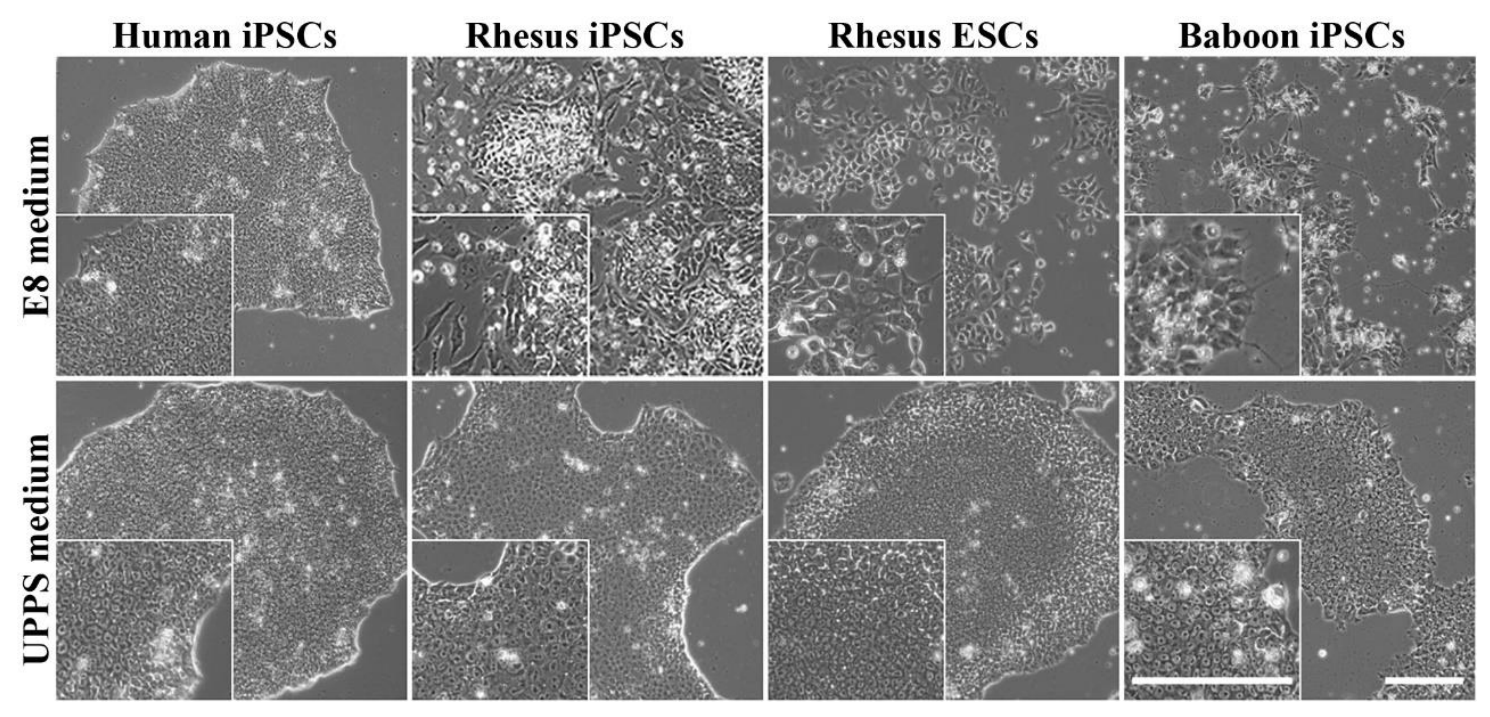




\section{Chapter 2: Reproducible Primate iPSC Generation, Cultivation, and Cardiac Differentiation under Chemically Defined Conditions}

Figure 2. Morphology of human, rhesus and baboon PSCs cultivated feeder-free in Essential 8 (E8) medium and Universal Primate Pluripotent Stem Cell (UPPS) medium. Human iPSCs grow as compact colonies showing a typical primed PSC morphology when cultivated in both, E8 and UPPS medium. In contrast, baboon and rhesus iPSCs differentiate spontaneously when cultivated in E8 medium but form undifferentiated compact colonies in UPPS medium. Adaptation of rhesus ESCs (Rh344.6) from feeder to feeder-free condition failed in E8 medium. Cultivated in UPPS medium, rhesus ESCs form compact undifferentiated colonies. Scale bars $=200 \mu \mathrm{m}$.

UPPS medium was tested for long-term maintenance of five rhesus (4 adult, 1 neonate), one adult baboon and two adult human iPSC lines. The iPSCs were cultured feeder-free on Geltrex-coated dishes under chemically defined conditions. All tested iPSC lines demonstrate similar morphologies with a high nucleus:cytoplasm ratio and distinct nucleoli (Fig.3). They express alkaline phosphatase (Fig.3C,G,K) and show a normal karyotype (Fig.3D,H,L). Only one female rhesus macaque line (DPZ_iRh25.4) shows a mosaic of $\mathrm{X}$ chromosome di-/trisomy, while the original fibroblasts of this animal, which were isolated from three independent sites (back skin, ventral skin, skin from the leg), were diploid. Immunostainings of the NHP-iPSCs show the expression of the pluripotency transcription factors NANOG and SALL4 (which are not encoded by the reprogramming plasmids), the cytoplasmic protein LIN28 as well as the surface markers SSEA4 and TRA-1-60 (Fig.S2). 

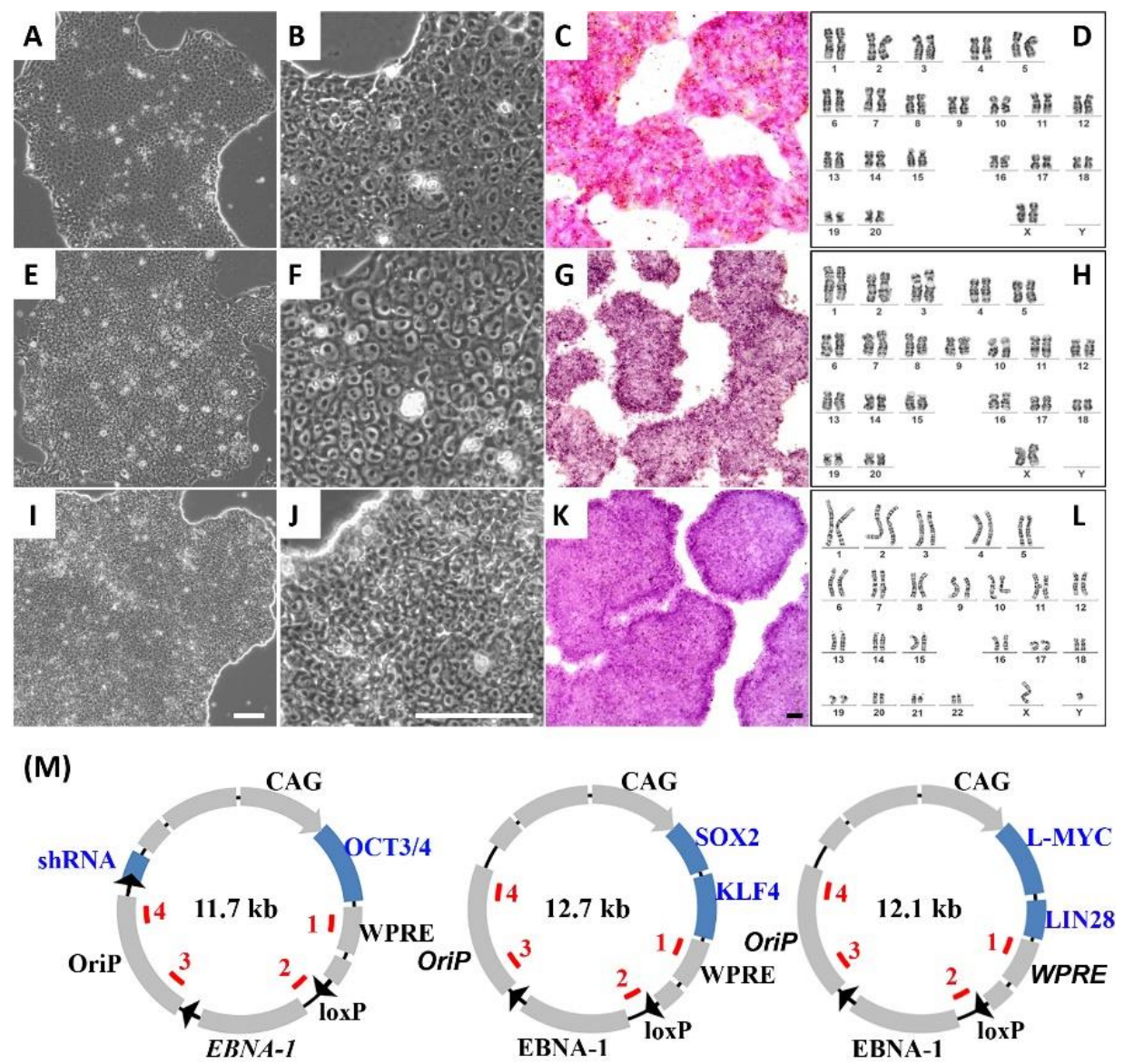

(N)

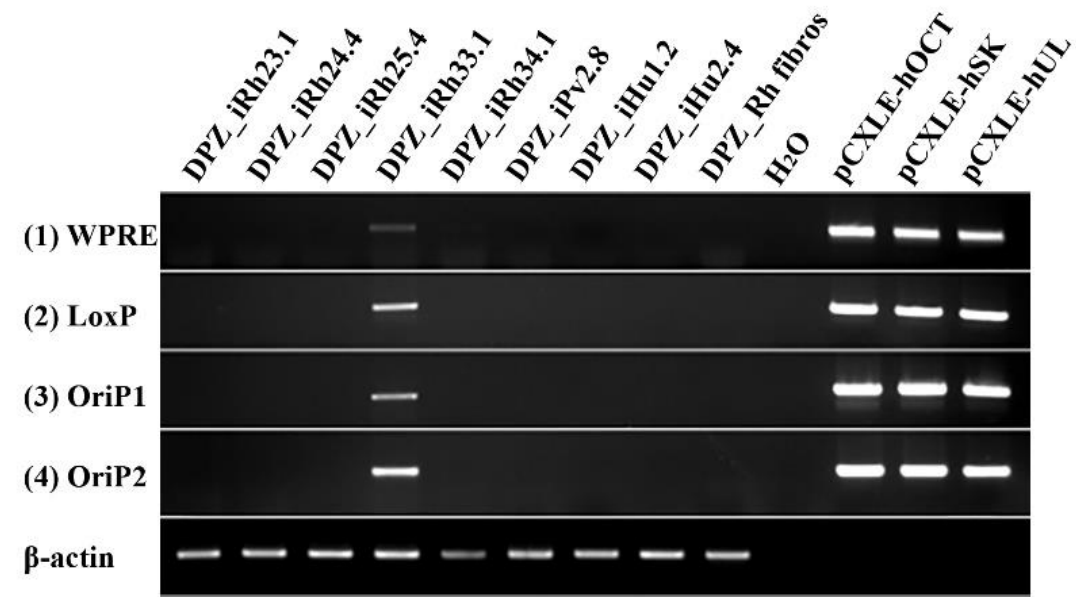

Figure 3. Generation and characterization of transgene-free NHP-iPSCs. (A), (B) Rhesus iPSCs cultivated under feeder-free conditions, (C) express alkaline phosphatase and (D) show a normal karyotype. (E), (F) Baboon iPSCs cultivated under feeder-free conditions, (G) express alkaline phosphatase and (H) show a normal karyotype. (I), (J) Human iPSCs cultivated under feeder-free conditions, $(\mathbf{K})$ express alkaline phosphatase and $(\mathbf{L})$ show a normal karyotype. Scale bar $=100 \mu \mathrm{m}$. (M) The primer-binding sites (1-4) within the plasmid DNA. Plasmid maps were modified from Okita et al. $2011^{15}$. (N) The absence of plasmid DNA was analyzed by PCR on gDNA using primers specific for four different regions of the plasmid DNA. Only the rhesus iPSC line DPZ_iRh33.1 shows integration of plasmid DNA. Rhesus fibroblasts and water were used as negative, and the three plasmids as positive controls. 


\section{Chapter 2: Reproducible Primate iPSC Generation, Cultivation, and Cardiac Differentiation under Chemically Defined Conditions}

Reverse transcription PCR analyses were performed for exogenous and endogenous expression of pluripotency factors (Fig.S3). Exogenous gene expression of OCT4A, $K L F 4$, and LIN28 was detectable two days after nucleofection in rhesus fibroblasts (Fig.S3). In line DPZ_iRh34.1, no exogenous expression was detectable at passage 11 or higher. Exogenous OCT4A expression was detectable at passage 11 in DPZ_iRh33.1, but became undetectable at later passages (Fig.S3). The endogenous expression of the pluripotency factors NANOG, OCT4A, SOX2, and LIN28 was observed in both cell lines in all three passages tested, as well as in the rhesus positive control ESC line. In rhesus fibroblasts, two days after nucleofection, the endogenous expression of NANOG, OCT4, SOX2, and LIN28 was not yet detectable. The loss of the reprogramming plasmid DNA from the generated iPSCs between passage 15 and 20 was confirmed via PCR with four primer pairs (Fig.3M, N). One of the iPSC lines (DPZ_iRh33.1) shows PCR signals even at higher passages suggesting the integration of a plasmid (Fig.3M). No plasmid DNA was detected in the human and in the other NHP-iPSC lines.

Altogether, after long-term culture in UPPS medium, the generated primate iPSC lines show the typical morphology of primed primate PSCs, have a normal karyotype, express (endogenous) pluripotency markers, and lost (except for line DPZ_iRh33.1) the reprogramming episomes.

\subsection{NHP-iPSCs cultured in UPPS medium are pluripotent}

In order to test whether the NHP-iPSCs are also functionally pluripotent under the newly established UPPS medium conditions, we differentiated them in vitro into embryoid bodies (Fig.4A,H). Plated bodies derived from rhesus (Fig.4A,D) and baboon (Fig.4E,H) iPSCs differentiated spontaneously into smooth muscle actin, $\alpha-$ fetoprotein and $\beta$-III-tubulin expressing cells, suggesting the development of cells representing the three germ layers. In vivo differentiation by teratoma formation corroborated the in vitro results. Teratomas contained, beside others, muscle, intestinal epithelial and neural tissues, representing mesoderm, endoderm, and ectoderm, respectively (Fig.4I, J and L). Immunostainings against neuronal-specific $\beta$-III-tubulin (Fig.4K,N) and SOX9 (primitive endodermal epithelium; Fig.5M) verified ectodermal and endodermal tissue, respectively. These data demonstrate that the NHP-iPSCs cultured under chemically defined UPPS medium conditions are pluripotent. 

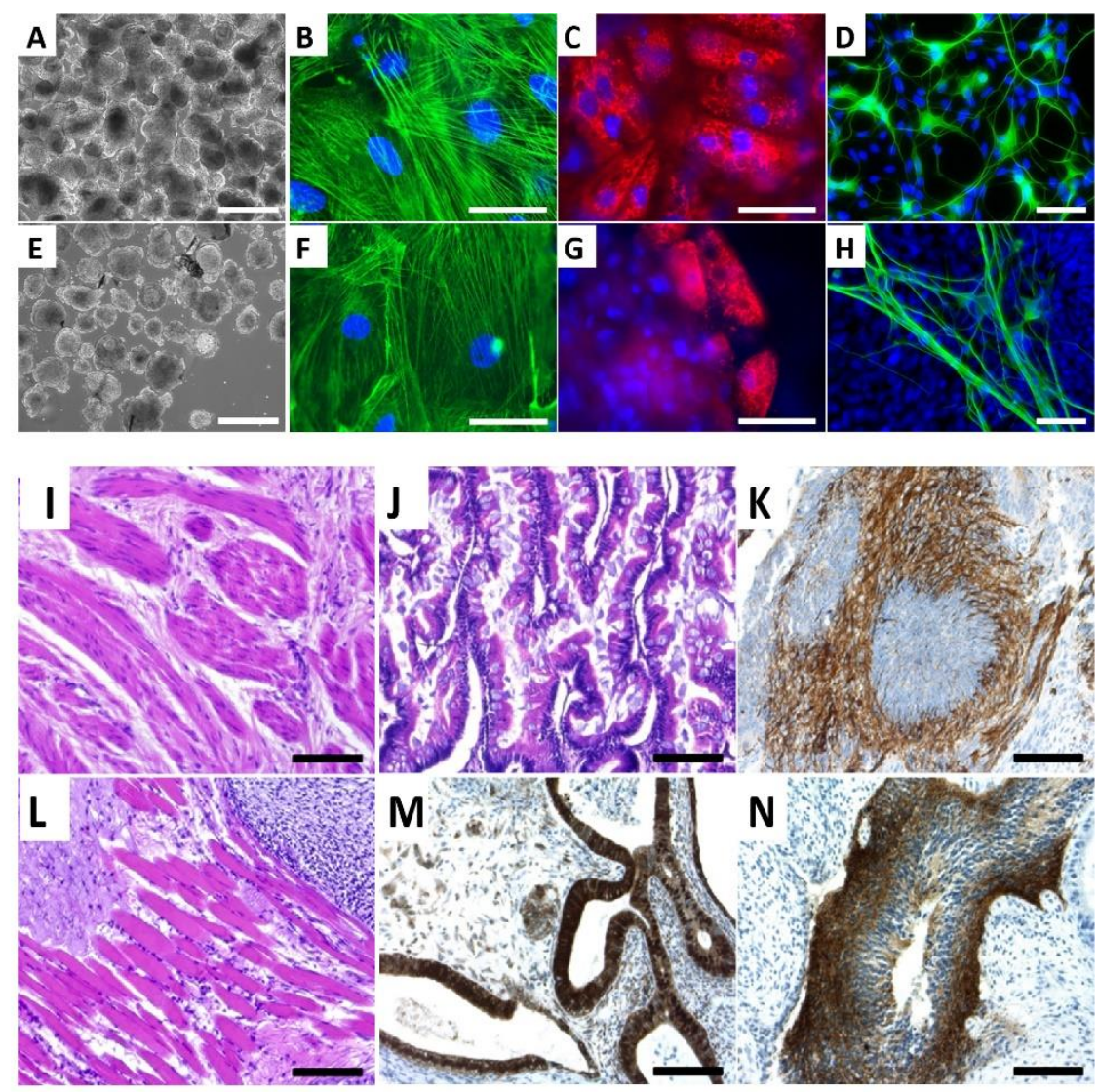

Figure 4. Differentiation potential of NHP-iPSCs. Embryoid body (EB) formation of (A, B, C and D) rhesus and $(\mathbf{E}, \mathbf{F}, \mathbf{G}$ and $\mathbf{H})$ baboon iPSCs in vitro. EBs differentiated spontaneously into different cell types, representing the three germ layers. (B) (F) mesoderm (smooth muscle actin), (C) (G) endoderm ( $\alpha$-fetoprotein) and (D) (H) ectoderm ( $($ III-tubulin). Scale bar (A) and $(\mathbf{E})=500 \mu \mathrm{m},(\mathbf{B})$ $(\mathbf{H})=50 \mu \mathrm{m}$. Teratoma formation of $(\mathbf{I}, \mathbf{J}, \mathbf{K})$ rhesus and $(\mathbf{L}, \mathbf{M}, \mathbf{N})$ baboon iPSCs after injection into immunodeficient mice. Teratomas contain containing derivatives of $(\mathbf{I}, \mathbf{L})$ mesoderm (muscle tissue), (J) endoderm (intestinal tissue), (M) endoderm (stained with SOX9) and (K), (N) ectoderm (neural tissue, stained with $\beta$-III-tubulin). Scale bar $=100 \mu \mathrm{m}$.

\subsection{NHP-iPSC-derived cardiomyocyte and EHM generation}

Several directed growth factor-free 2D monolayer cardiac differentiation protocols have been established for human PSCs 3233353637 . We first tried to adopt the small molecule-based differentiation protocols lacking growth factors to the rhesus and baboon iPSCs. We tested Chir99021 and Wnt agonists IWR-1 or IWP-2 in different concentrations and timings in different media (see Suppl, detailed in Unsuccessful growth factor-free NHP cardiomyocyte differentiation protocol). However, most of these cardiac differentiation experiments failed for most of NHP-iPSC lines; only sporadically changing subsets of the NHP-iPSCs responded to the differentiation stimuli. In contrast, the human cells efficiently and robustly differentiated into cardiomyocytes. An efficient and reproducible cardiac differentiation protocol working for all NHP-iPSC lines based only on small molecules, could not be 
established. This highlights the difference between human and NHP-iPSC cardiomyocyte differentiation. Furthermore, these data suggest inter iPSC line variability.

We then combined the small molecule protocol with BMP4 and Activin A (Fig.5A). With this hybrid method, we successfully and robustly differentiated NHP-iPSCs into cardiomyocytes. First beating cardiomyocytes from rhesus, baboon and human cells could be observed between day 7-8 of the differentiation protocol. Flow cytometric analyses of cTNT positive cells revealed average cardiac differentiation efficiencies between $53 \%$ and $72 \%$ at day 12 of differentiation (rhesus [53\%], baboon [70\%], human [72\%]; Fig.S4).

(A)

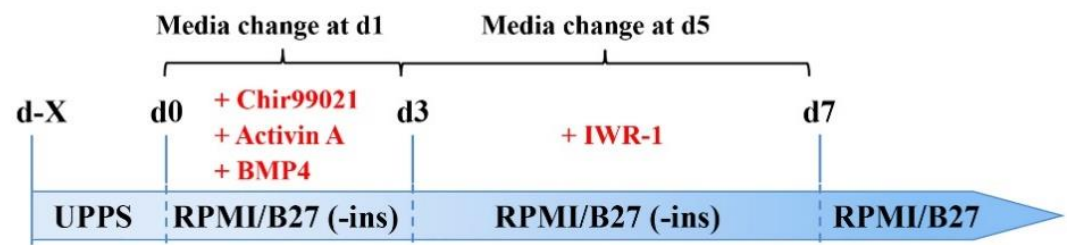

(B)

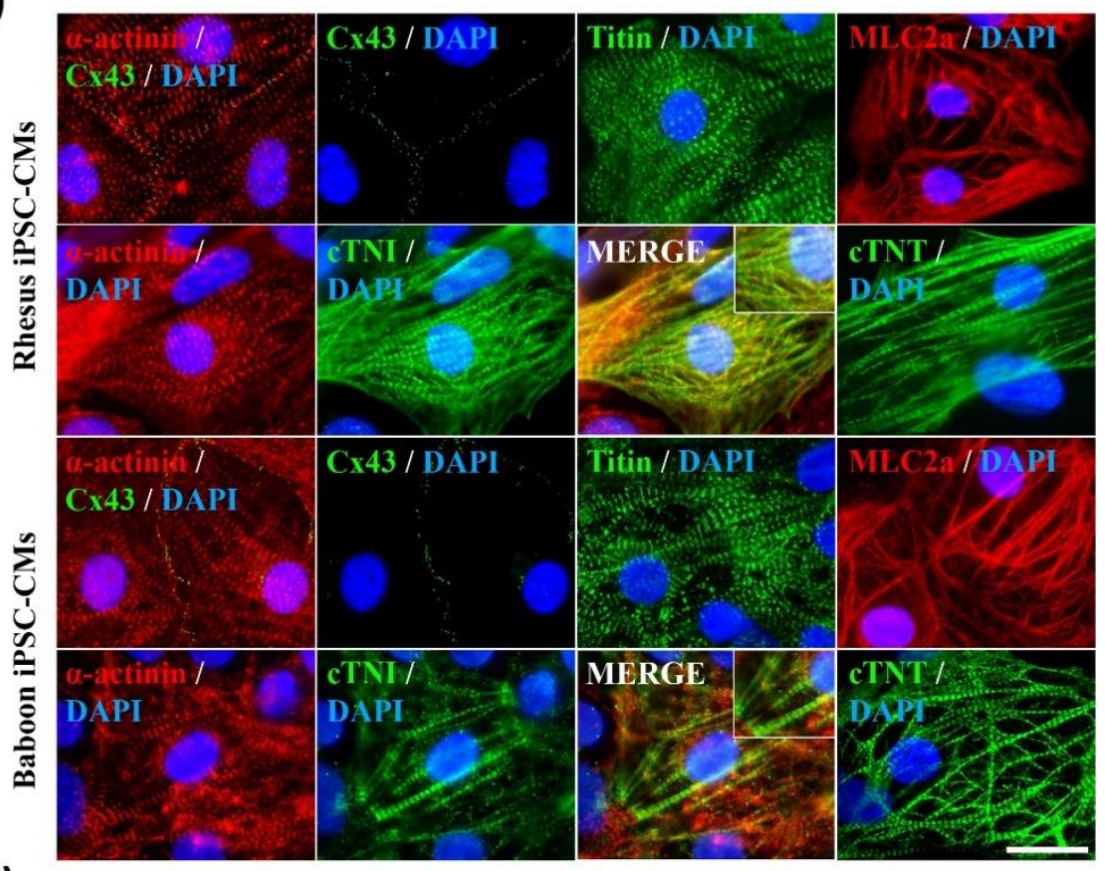

(C)

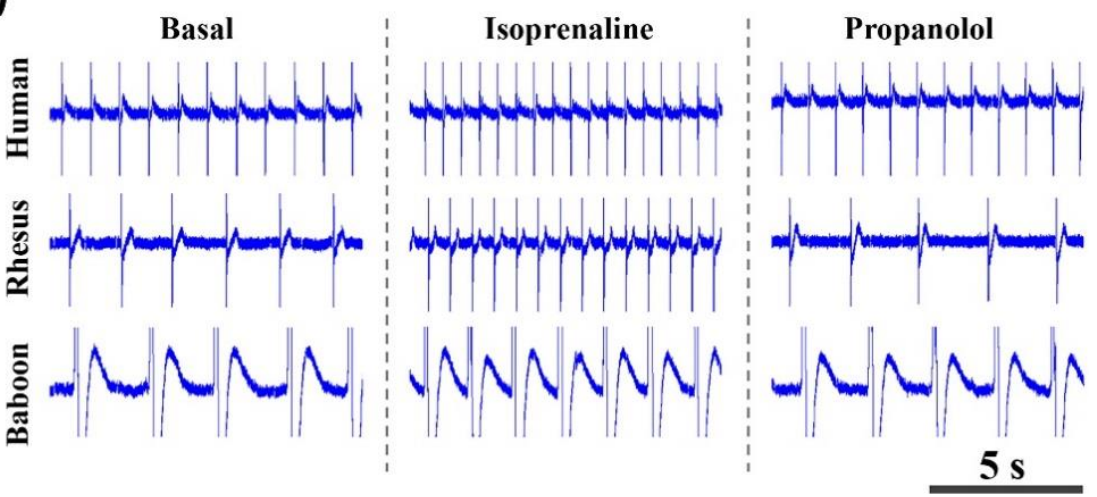




\section{Chapter 2: Reproducible Primate iPSC Generation, Cultivation, and Cardiac Differentiation under Chemically Defined Conditions}

Figure 5. Directed cardiac differentiation of human and NHP-iPSCs. (A) Scheme of the cardiac differentiation protocol for human, rhesus and baboon cells. (B) The structure and morphology of the cardiomyocytes are visualized by immunostainings of cardiac-specific genes: $\alpha$-actinin, cardiac troponin I (cTNI), cardiac troponin T (cTNT), connexin 43 (Cx43), myosin light chain a (MLC2a) and titin. Scale bars $=20 \mu \mathrm{m}$. (C) The cardiomyocytes respond to isoprenaline (increased beating frequencies compared to basal) and propanolol (decreased beating frequencies compared to isoprenaline back to basal). Beating frequencies were analyzed by measuring the field potentials with the microelectrode array (MEA) system.

For further characterize the cardiomyocyte populations, differentiations were purified by metabolic selection using lactate and further cultivated up to two months. The morphology, size, structure as well as cardiac-specific gene expression of 2-month-old rhesus and baboon iPSC-derived cardiomyocytes were similar and showed the typical immature, fetal-like morphology as known for human iPSC-derived cardiomyocytes (Fig.5B). Immunostainings of $\alpha$-actinin, cardiac troponin T, cardiac troponin I and titin show an immature net-like striated pattern of sarcomere structures (compared to adult cardiomyocytes with a parallel-organized pattern). The NHP cardiomyocytes express the gap junction protein connexin 43, supporting electrical cell-to-cell coupling (Fig.5B). As an initial functional analysis, field potential measurements of the human and NHP-iPSC-derived cardiomyocytes were measured in the 2D microelectrode array (MEA) set-up after treatment with the non-selective $\beta 1$ - and $\beta 2$ - adrenoreceptor agonist isoprenaline $(100 \mathrm{nM})$. In all three species, isoprenaline caused an increase in the beating frequencies of the cardiomyocytes relative to basal (untreated) conditions $(107.39 \pm 121.25,122.28 \pm 68.90$, and $16.7 \pm 54.38 \%$ change in the beating frequencies for human, rhesus macaque, and baboon iPSC-CM, respectively; Fig.5C). After 5 minutes of treatment with isoprenaline, propanolol $(2 \mu \mathrm{M})$ was consecutively added to the medium. This $\beta$-blocker reversed the chronotropic effect of isoprenaline back to basal conditions in all species.

In order to verify the functionality of the iPSC-derived cardiomyocytes in a biologically relevant 3D tissue setting, we tested their functionality by engineered heart muscle $(\mathrm{EHM}){ }^{38}$. Beating EHM were generated from one human (DPZ_Hs_1.2) and two Rhesus macaque iPSC lines (DPZ_iRh24.4, DPZ_iRh33.1). Before EHM generation, the percentage of cardiomyocytes in the cultures was found to be around $90 \%$ or higher (evaluated by FACS, $\alpha$-actinin staining) (Fig.6A). The spontaneous beating frequency of the human EHM was $50.3 \pm 10.1$ (mean \pm SD) beats per minute (bpm), while the respective values for DPZ_iRh24.4 and DPZ_iRh33.1 were $87.5 \pm$ 8.67 and $102.5 \pm 3.5$. Corresponding to the beating frequency, the time to $90 \%$ contraction and 50\% relaxation was longest in the human EHM, while it was shortest in the line DPZ_iRh33.1 (Fig.6C, D). Organotropic responses to calcium and catecholamine stimulation were evaluated by the addition of increasing concentrations of extracellular calcium and acute isoprenaline, respectively. All EHMs showed a dose-dependent contraction force response to increasing extracellular calcium levels (Fig.6E). While the human EHM showed a very strong response, the rhesus EHMs 


\section{Chapter 2: Reproducible Primate iPSC Generation, Cultivation, and Cardiac Differentiation under Chemically Defined Conditions}

responses were moderate. To test the responses of the EHMs to beta-adrenergic stimulation, isoprenaline was added to the medium, and the \% change in the force of contraction was measured (Fig.6F). The human EHM showed a 75\% change, while the DPZ_iRh24.4 EHM exhibited a 90\% and the DPZ_iRh33.1 EHM a 40\% change. In summary, NHP-iPSCs cultured in UPPS medium can form functional cardiomyocytes that physiologically respond to external stimuli as the human iPSCderived EHM.

In conclusion, we have established a reproducible, fast, and universal cardiac differentiation protocol for primate iPSCs under chemically defined conditions.

(A)

(C)
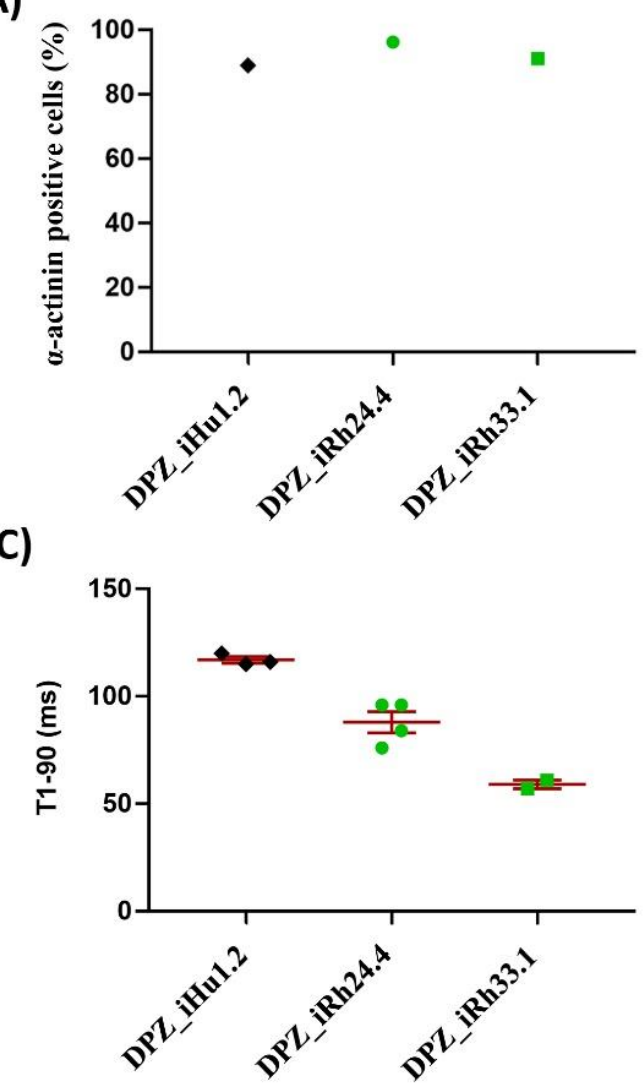

(E)

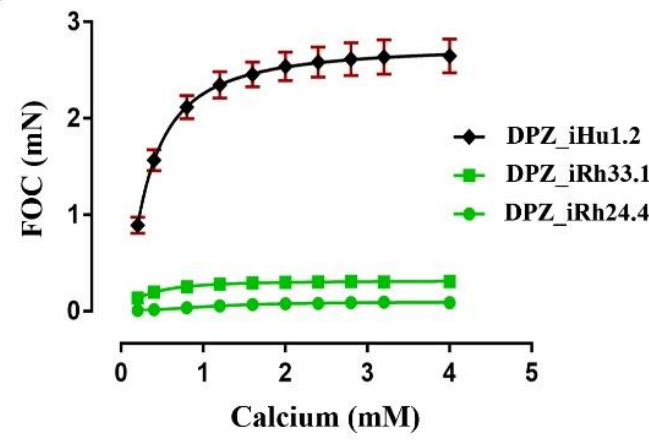

(B)

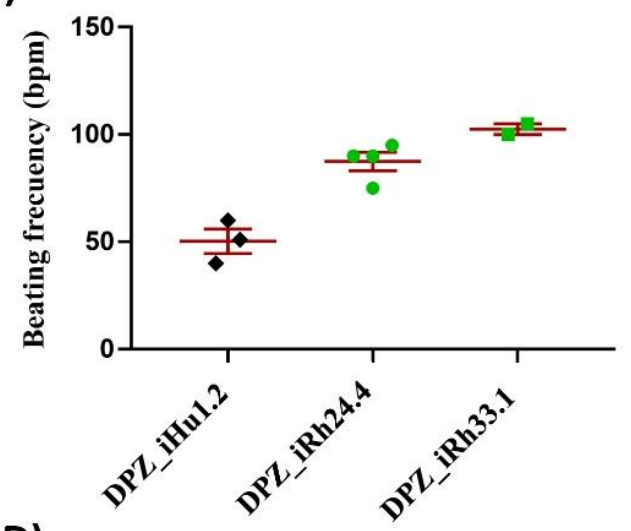

(D)

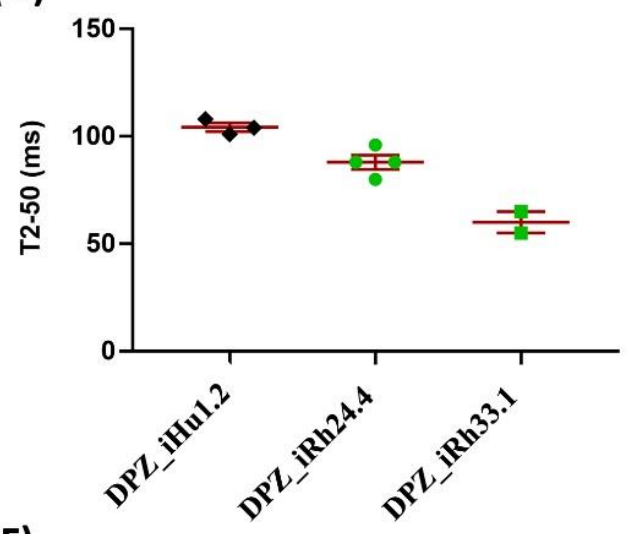

(F)

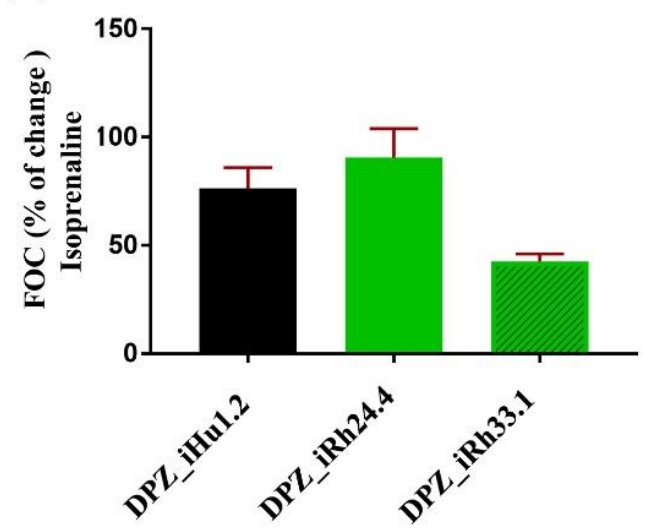




\section{Chapter 2: Reproducible Primate iPSC Generation, Cultivation, and Cardiac Differentiation under Chemically Defined Conditions}

Figure 6. Functional Characterization of primate EHM. (A) The percentage of cardiomyocytes after metabolic selection and before EHM generation, for one human (iHs 1.2) and two Rhesus (DPZ_iRh24.4, DPZ_iRh33.1) lines. (B) EHM beating frequency (bpm) after four weeks of EHM culture. (C) The time to $90 \%$ contraction of the EHMs reversely correlates with the beating frequency. (D) The time to 50\% relaxation correlates with the $90 \%$ contraction time. (E) Analysis of the force of contraction of EHMs by addition of extracellular calcium in serum-free conditions. The analysis was performed for one human (DPZ_iHs1.2), and two Rhesus macaque (DPZ_iRh33.1, and DPZ_iRh24.4) lines. Despite differences in the absolute values, all three lines show a response to increased calcium concentrations. (F) Primate EHM inotropic response to acute isoprenaline $(1 \mu \mathrm{M}, \mathrm{ISO})$ stimulation given as \% change in the force of contraction.

\section{Materials and methods}

\subsection{NHP species and ethics statement}

Two large NHP species the rhesus macaque (Macaca mulatta) and the olive baboon (Papio anubis), were included in the study. Both species show a close phylogenetic relationship to humans (Fig.S1), and are favorable NHP species in cardiovascular research 20213940 . The range of the body weights of adult rhesus monkeys is 4 to $14 \mathrm{~kg}$ and of the olive baboon $\sim 10$ to $37 \mathrm{~kg}$. The permission and ethical approval to take skin biopsies from the macaques were obtained under the license number 42502-0416/2370 from the Niedersächsisches Landesamt für Verbraucherschutz und Lebensmittelsicherheit (LAVES). The baboon skin sample was made available to the Platform Degenerative Diseases of the German Primate Center (DPZ) during necropsy of the animal by veterinarian pathologists, which does not require specific permission. The DPZ runs self-sustaining colonies of different primate species including macaques and baboons and is registered and authorized by the veterinary governmental authorities (Reference number: 122910.3311900, PK Landkreis, Göttingen).

\subsection{Isolation and cultivation of NHP and human skin fibroblasts}

To cover a broader spectrum of cell donors and to ensure reproducibility, skin fibroblasts from five rhesus macaques (one newborn and four adult individuals including males and females) and one adult female baboon were isolated from outgrowths from small skin samples. Cells were cultivated in fibroblast cultivation medium (89 ml DMEM, $10 \mathrm{ml}$ fetal bovine serum (FBS), $1 \mathrm{ml}$ 100x non-essential amino acids (NEAA), $0.1 \mathrm{mM}$ 2-mercaptoethanol; all Thermo Fisher) supplemented with $10 \mathrm{ng} / \mathrm{ml} \mathrm{bFGF}$ (PeproTech) and penicillin-streptomycin (Thermo Fisher) on 6 $\mathrm{cm}$ cell culture dishes (CytoOne). Cell outgrowths were split using $0.25 \%$ trypsin/EDTA (Thermo Fisher) and propagated in fibroblast medium containing bFGF but without further antibiotic treatment. Human skin fibroblasts were purchased from Lonza (CC-2511, lot 0000545147 [male], lot 0000490824 [female]) and cultivated under the same conditions. 


\subsection{Reprogramming NHP and human skin fibroblasts}

Skin fibroblasts were nucleofected with the three episomal vectors ${ }^{15} p C X L E$ hOCT3/4-shp53-F (\#27077), pCXLE-hSK (\#27078) and pCXLE-hUL (\#27080) using the 4D-Nucleofector Core Unit and the P2 Primary Cell Solution (Lonza). We transfected $2 \mu \mathrm{g}$ of each plasmid per $6 \times 10^{5}$ cells and plated them onto 6-well plates ( 3 x $10^{5}$ cells per well; Greiner Bio-One) in fibroblast cultivation medium supplemented with $10 \mathrm{ng} / \mathrm{ml}$ bFGF, $5 \mu \mathrm{M}$ Pro-survival compound (Merck) and penicillin-streptomycin. The next day, the medium was replaced by fresh fibroblast medium containing $10 \mathrm{ng} / \mathrm{ml} \mathrm{bFGF}$ and $0.5 \mathrm{mM}$ sodium butyrate (Sigma-Aldrich). Medium was changed daily. At day 7 after transfection, the cells were spilt onto Geltrex $(0.16 \mathrm{mg} / \mathrm{ml}$; Thermo Fisher) coated 6-well plates. At day 8, fibroblast cultivation medium was replaced by Essential 8 medium (Thermo Fisher) supplemented with $0.5 \mathrm{mM}$ sodium butyrate until day 11 . From day 12 on, the transfected cells were cultivated in Essential 8 medium only. Appearing iPSC colonies were picked manually and transferred into 12-well plates (Greiner Bio-One). For continuous cultivation and expansion, the medium was replaced by our newly developed universal primate pluripotent stem cell (UPPS) medium (see below).

For quantification of the reprogramming efficiency, the skin fibroblasts of three rhesus macaques and two humans were transfected and cultured as described above. The nucleofected fibroblasts were split into three wells of a 6-well plate at day 7 . The appearing colonies were stained for alkaline phosphatase and counted at day 20, 30 and 40 after nucleofection (colonies counted in one well at each time point). Alkaline phosphatase activity was detected using an alkaline phosphatase staining kit (SigmaAldrich).

\subsection{Cultivation of human and NHP-PSCs}

Human- and NHP-PSCs were cultivated in UPPS medium, which is based on StemMACS iPS Brew XF (Miltenyi Biotec). This chemically defined human PSC cultivation medium was supplemented with $1 \mu \mathrm{M}$ inhibitor of Wnt response-1 (IWR1, Sigma-Aldrich) and $0.5 \mu \mathrm{M}$ Chir99021 (Merck). The stem cells were cultured on Geltrex coated $(0.16 \mathrm{mg} / \mathrm{ml})$ culture dishes at $37^{\circ} \mathrm{C}$ and $5 \% \mathrm{CO} 2$. The cells were split every 3-4 days onto new culture dishes using Versene solution (Thermo Fisher). After splitting, the UPPS medium was supplemented with $5 \mu \mathrm{M}$ Pro-survival compound for one day.

\subsection{In vivo and in vitro differentiation}

For in vivo differentiation experiments (teratoma formation assay), human and NHPiPSC colonies were treated with collagenase type IV (200 U/ml; Worthington) for 10 min at $37{ }^{\circ} \mathrm{C}$, washed and dissected into small clusters using a cell scraper (Sarstedt) 


\section{Chapter 2: Reproducible Primate iPSC Generation, Cultivation, and Cardiac Differentiation under Chemically Defined Conditions}

and by pipetting smoothly up and down. The cell clusters were centrifuged and resuspended together with irradiated mouse embryonic fibroblasts in $140 \mu$ UPPS medium supplemented with $5 \mu \mathrm{M}$ Pro-survival compound and $120 \mu \mathrm{l}$ Geltrex $(0.16$ $\mathrm{mg} / \mathrm{ml}$ ). $100 \mu \mathrm{l}$ of this suspension was then injected subcutaneously into SCID/beige (C.B-17/IcrHsd-scid-bg) mice. Earliest teratoma formation was detected one month post-injection. The teratomas were fixed with Bouin's fixative for $24 \mathrm{~h}$ and embedded into paraffin. Histological sections were stained with hematoxylin and eosin (Merck) or with antibodies against SOX9 and $\beta$-tubulin III (Suppl. Table 1).

NHP-iPSCs were also differentiated in vitro using the embryoid body (EB) formation method. Briefly, after treatment with collagenase type IV, cell clusters were cultivated in suspension in UPPS medium for $24 \mathrm{~h}$ giving them time to form EBs. The next day, the medium was replaced by differentiation medium (79 $\mathrm{ml} \mathrm{IMDM}$, Thermo Fisher), $20 \mathrm{ml} \mathrm{FBS}, 1 \mathrm{ml}$ 100x NEAA, $450 \mu \mathrm{M}$ 1-thioglycerol (Sigma-Aldrich)). At day 8, the EBs were plated onto $0.1 \%$ gelatin-coated coverslips (Menzel) and fixed for immunostaining at day 25.

\subsection{Cardiac differentiation}

Different protocols for cardiac differentiation established for human PSCs were tested for our rhesus and baboon iPSCs. The three tested basic approaches (see Suppl, detailed in Unsuccessful growth factor-free NHP cardiomyocyte differentiation protocol) were based on the small molecule Chir99021 with and without the growth factors, BMP4 and Activin A. In contrast to the human, cardiac differentiation growth factor-free protocols did not work for NHP. However, the hybrid differentiation method, including Chir99021, Activin A and BMP4, was successful. When reaching confluency of about $80-90 \%$, the UPPS medium was replaced by mesodermal induction medium (RPMI 1640, B27 without insulin, $1 \mathrm{mM}$ sodium pyruvate (Thermo Fisher), $200 \mu \mathrm{M}$ L-ascorbic acid 2-phosphate, $1 \mu \mathrm{M}$ Chir99021, $9 \mathrm{ng} / \mathrm{ml}$ Activin A (Miltenyi Biotec, premium grade), $5 \mathrm{ng} / \mathrm{ml}$ BMP4 (Miltenyi Biotec, premium grade). Medium was changed after $24 \mathrm{~h}$. At day 3, medium was replaced by cardiac induction medium (RPMI 1640, B27 supplement without insulin, $1 \mathrm{mM}$ sodium pyruvate, 200 $\mu \mathrm{M}$ L-ascorbic acid 2-phosphate, $5 \mu \mathrm{M}$ IWR-1). Medium was changed at day 5. At day 7, the medium was replaced by cardiomyocyte cultivation medium (RPMI 1640, B27 with insulin, $200 \mu \mathrm{M}$ L-ascorbic acid 2-phosphate). The cultures were monitored daily for contractile activity. To increase the yield of pure cardiomyocytes, the cells were metabolically selected with lactate. Around 20 days post differentiation, cells were plated in Geltrex coated 6-well plates. Metabolic selection was achieved on 4-5 days of cultivation in medium containing lactate but no glucose (RPMI 1640 without glucose (Thermo Fisher), $4 \mathrm{mM}$ lactate/HEPES solution, $0.2 \mathrm{mg} / \mathrm{ml} \mathrm{L}$-ascorbic acid 2phosphate, $0.5 \mathrm{mg} / \mathrm{ml}$ recombinant human albumin). After cardiomyocyte selection, the medium was changed again to cardiomyocyte cultivation medium. 


\subsection{Immunostaining}

Cells were cultured on coverslips and fixed with $4 \%$ (w/v) paraformaldehyde (Merck) in DPBS (Thermo Fisher) for $20 \mathrm{~min}$ at room temperature and subsequently washed with DPBS. After blocking with $1 \%$ bovine serum albumin (BSA; Thermo Fisher), the cells were incubated with primary antibodies (Suppl. Table 1) at $4{ }^{\circ} \mathrm{C}$ overnight. Permeabilization for staining intracellular proteins was achieved by treating with $0.1 \%$ Triton X-100 (Sigma-Aldrich). After washing with DPBS, the secondary antibodies were incubated (Suppl. Table 2) for $1 \mathrm{~h}$ at $37^{\circ} \mathrm{C}$. Cell nuclei were stained with $1 \mu \mathrm{g} / \mathrm{ml}$ DAPI (Sigma-Aldrich). The coverslips were finally mounted with Fluoromount-G (eBioscience) and analyzed with a fluorescence microscope (Zeiss). Immunohistochemical staining of the teratomas was performed as described recently 41 .

\subsection{Flow cytometry}

At day 12 after initiation of differentiation, human and NHP iPSC-derived cardiomyocytes were treated with $0.25 \%$ trypsin/EDTA, flushed through a $40 \mu \mathrm{M}$ cell strainer (Falcon) and single cells were fixed with $4 \%$ (w/v) paraformaldehyde for 10 min. After blocking with $1 \% \mathrm{BSA}$ at $4^{\circ} \mathrm{C}$, the cells were incubated with FITC-labeled cTNT antibody (Suppl. Table 1) at $4^{\circ} \mathrm{C}$ overnight. The next day, cells were washed and resuspended in $100 \mu 1$ flow cytometry buffer (0.5 \% BSA, 2 mM EDTA (Roth)). Unstained cells from each cell suspension and appropriate isogenic controls were included as negative controls in each case. Flow cytometric analyses (10,000 cells per sample) were performed with SH800S Cell Sorter using a $130 \mu \mathrm{m}$ sorting chip (Sony) according to the manufacturer's instructions.

\subsection{DNA and RNA isolation and polymerase chain reaction}

Genomic DNA from snap-frozen cell pellet samples (between passage 15 and 25) was isolated using the DNeasy Blood \& Tissue Kit (Qiagen) according to the manufacturer's instructions. To confirm the loss of episomal plasmids in the generated iPSCs, we performed a standard polymerase chain reaction (PCR) using plasmidspecific primer pairs (Suppl. Table 3), which are complementary to four different regions present in all three plasmids (Fig.3). One PCR reaction $(50 \mu 1)$ was performed using 50-100 ng of genomic DNA, using Taq DNA Polymerase with Standard Taq Buffer (New England Biolabs), following the manufacturer's instructions.

For gene expression analyses, total RNA from snap-frozen cell pellets was isolated using NucleoSpin RNA Plus isolation kit (Macherey-Nagel), treated with RNase-free DNase (Qiagen) and subsequently transcribed into cDNA with Omniscript RT kit (Qiagen) according to the manufacturer's instructions. The cDNA was then amplified 


\section{Chapter 2: Reproducible Primate iPSC Generation, Cultivation, and Cardiac Differentiation under Chemically Defined Conditions}

using Taq DNA polymerase with Standard Taq Buffer (New England Biolabs). All oligonucleotides (Sigma-Aldrich) are listed in Suppl. Table 3.

\subsection{Karyotyping}

Karyotyping was done as described previously in Rodriguez-Polo I et al., $2015^{42}$ (Chapter 1).

\subsection{Microelectrode array (MEA) measurements}

The iPSC-derived cardiomyocytes were treated with $0.25 \%$ trypsin/EDTA and plated onto Geltrex coated 6-well MEAs (Multichannel Systems). After 4-7 days of recovering, field potentials of the cardiomyocytes were measured using the MEA2100$2 \times 60$-System at $38^{\circ} \mathrm{C}$, which is the physiological body temperature of macaques. After basal field potential measurements, the cells were treated with the non-selective $\beta$ adrenoreceptor agonist isoprenaline (100 nM; Sigma-Aldrich) followed, after 5 min by propranolol $(2 \mu \mathrm{M})$. The effect of these drugs on the beating frequency was detected by continuously measuring the field potentials of the cardiomyocytes. Data were acquired and analyzed using MC_Rack software (Multichannel Systems).

\subsection{EHM generation}

Engineered heart muscle (EHM) generation was performed according to Tiburcy, M. et al. $2017^{38}$. In brief, primate cardiomyocytes after selection were mixed with human foreskin fibroblast (HFFs, American Type Culture Collection) (70:30\%). Cell solution was reconstituted in $\mathrm{pH}$-neutralized medical-grade bovine collagen (0.4 mg per EHM; LLC Collagen Solutions) and concentrated serum-free medium (2× RPMI, 8\% B27 without insulin, penicillin [200 U/ml], streptomycin [200 $\mu \mathrm{g} / \mathrm{ml}]$ ). Afterwards, EHMs were cultured for 3 days in IMDM with 4\% B27 without insulin, 1\% NEAA, $2 \mathrm{mM}$ glutamine, $300 \mu \mathrm{M}$ ascorbic acid, IGF1 (100 ng/ml; AF-100-11), FGF-2 (10 ng/ml; AF-100-18B; PeproTech), VEGF165 (5 ng/ml; AF-100-20; PeproTech), TGF- $\beta 1$ (5 $\mathrm{ng} / \mathrm{ml}$; AF-100-21C; PeproTech), penicillin (100 U/ml), and streptomycin (100 $\mu \mathrm{g} / \mathrm{ml})$. After 3 days, the EHM was transferred to flexible holders, and 4 weeks later, EHM analysis was performed as described in ${ }^{38}$.

\section{Discussion}

Pluripotent stem cells, including iPSCs, revolutionize different fields of biomedicine, including potential treatments for until the moment incurable diseases. In fact, several late preclinical animal studies involving NHP have been already conducted 212340 . The promising findings in the mention projects reflect in the first clinical trials of PSCbased ATMPs, e.g., for the treatment of age-related macular degeneration ${ }^{43}$ and Parkinson's disease. Expectations are high that in the future PSCs might be used 


\section{Chapter 2: Reproducible Primate iPSC Generation, Cultivation, and Cardiac Differentiation under Chemically Defined Conditions}

routinely as a therapeutic agent in the clinic. However, these therapies are currently experimental, and there are still many open questions. Most of them can be adequately addressed only using NHP due to their close phylogenetic relationship to humans, which is reflected by generally comparable anatomy, immune systems, and genetic and transcriptomic constitution as well as life span ${ }^{246}$. Allogeneic and autologous transplantations, using NHP as model organisms, may also provide a deeper understanding of the respective immunological responses and the corresponding immunosuppression regimens for PSC-derived transplants in humans (for review see $\mathrm{Wu}$ et al., ${ }^{44}$ ). In the present study, we developed a new cost-efficient and, most notably, robustly reproducible reprogramming protocol for the generation of integration-free NHP-iPSCs. Moreover, we established a universal cultivation method under chemically defined conditions for NHP and human PSCs to ensure optimal comparability between cell lines from different primate species and their responses to differentiation cues and immunological challenges. This will improve the translatability of findings from NHP to the clinics.

We reprogrammed cells from five rhesus macaques, one baboon, and two humans to demonstrate the broad applicability of our reprogramming method. To our knowledge, most of the relatively few NHP-iPSC lines generated until now (including macaque, baboon, gorilla, bonobo and common marmoset) were typically reprogrammed using a method involving genomic integration of the reprogramming vectors $2728293045-49$; or genomic integration followed by the excision of the lentiviral vector leaving a genetic tag in the host genome ${ }^{50}$ 51. Human iPSCs with genomic integration of the reprogramming vectors are not acceptable for therapeutic applications in patients. Regarding the translational value of NHP, we decided to generate genomically nonmodified primate iPSCs using episomal plasmid vectors. The episomal system involves p53 suppression and non-transforming L-MYC instead of c-MYC ${ }^{15}$. In all of the iPSC lines except one (iRh33.1), we could not detect the reprogramming vector DNA indicating that the iPSC lines are integration-free. Our results (one out of eight iPSC lines with integration) are in line with the original publication of the episomal system where two out of seven human cell lines showed vector DNA integration into the host genome ${ }^{15}$.

An interesting observation was that the NHP cells were less efficient in reprogramming compared to the human cells. The reason for this is currently unknown. One possible explanation could be that the human reprogramming factors may work less efficient in the NHP than in the human cells. However, the reprogramming factors are highly conserved between humans and rhesus macaques with identities on the protein level of 99\% (OCT4, SOX2, KLF4, LIN28) and 96\% (L-MYC) (Suppl. Table 4). Furthermore, the human p53 shRNA sequence is $100 \%$ complementary to the respective rhesus sequence. In a previous study, we used marmoset sequences cloned into a stably expressing piggyBac transposon to reprogram neonatal marmoset fibroblasts and obtained only very few primary colonies ${ }^{29}$. Recently, we also generated baboon iPSCs using the same piggyBac vector and obtained also only a 


\section{Chapter 2: Reproducible Primate iPSC Generation, Cultivation, and Cardiac Differentiation under Chemically Defined Conditions}

rather small number of primary colonies (Chapter 1$)^{42}$. Finally, it has been shown that mouse factors are sufficient to efficiently reprogram human cells and vice versa ${ }^{2152}$. Taken together, all this strongly argues against the hypothesis that the human reprogramming factors are responsible for the low reprogramming efficiencies of the rhesus (and the baboon) cells compared to the human cells. Further investigations are necessary for final conclusions. However, our standardized primate (including human) reprogramming protocol in combination with single-cell -omics may help to identify the differences between human and NHP reprogramming on the molecular level.

Using the same approach as for human cells, we reprogrammed NHP fibroblasts. The generated cells were maintained in a chemically defined medium, comparable to clinically relevant conditions. To our knowledge, this is the first report showing successful NHP-iPSC generation and maintenance without the support of feeder cells 272930464851535455 . The generation of human and NHP-iPSCs was successful in our study using the well-defined Essential 8 cultivation medium developed for human stem cell culture ${ }^{56}$. As expected, the generated human iPSCs could be cultivated in this medium while keeping their pluripotent state. However, the undifferentiated long-term cultivation of the NHP-iPSCs was not possible due to permanent differentiation of the culture. To exclude that our NHP-iPSCs were incompletely reprogrammed, we tried to cultivate the rhesus ESC line Rh366.4 ${ }^{34}$ in Essential 8 media. However, the rhesus ESC line also differentiated spontaneously when cultivated feeder-free in Essential 8 medium. The same finding was made using StemMACS iPS Brew XF. This showed that the commercial media alone are not sufficient to keep rhesus and baboon PSCs in a stable undifferentiated pluripotent state, while the cells could be maintained feederfree using the UPPS medium. In addition, we also tested the supplementation of both cultivation media with inhibitor of Wnt response-1 (IWR-1), a Wnt signaling inhibitor, amongst other factors. It was shown that the addition of IWR-1 could optimize the derivation and long-term cultivation of mouse epiblast stem cells (EpiSCs) in combination with FGF2 in N2B27 media ${ }^{57}$. Mouse EpiSCs are considered as the in vitro counterparts of human (primed) ESCs. Wu et al. postulated the supportive effect of IWR-1 also on human ESCs, rhesus PSCs and chimpanzee iPSCs ${ }^{57}$. The combined addition of IWR-1 and bone morphogenetic protein 4 (BMP4) to Essential 8 and StemMACS iPS-Brew XF media was tested as a second condition. It was shown that BMP4 is secreted by mouse embryonic fibroblasts and was identified as pluripotencyand self-renewal promoting factor for mouse ESCs ${ }^{58}$. As a third condition, we tested IWR-1 combined with the Wnt pathway activator Chir99021. Kim and colleagues could show that the dual administration of Chir99201 and Wnt inhibitor XAV939 or Chir99201 and IWR-1 allowed long-term maintenance of undifferentiated mouse EpiSCs as well as human ESCs. These cells were routinely maintained on mouse embryonic fibroblasts in semi-defined cultivation media using Knockout serum replacement ${ }^{59}$. Of all tested conditions, the combination of Chir99201 and IWR-1 in StemMACS iPS Brew XF medium turned out to be the only condition in our study facilitating consistent undifferentiated long-term self-renewal (> 50 passages) of rhesus, baboon, and human iPSCs. Eventually, we decreased the concentrations from 


\section{Chapter 2: Reproducible Primate iPSC Generation, Cultivation, and Cardiac Differentiation under Chemically Defined Conditions}

$3 \mu \mathrm{M}$ Chir99201 and $2.5 \mu \mathrm{M}$ IWR-1, as originally published by Kim and colleagues, to $0.5 \mu \mathrm{M}$ Chir99021 and $1 \mu \mathrm{M}$ IWR-1. We named our new formulation universal primate pluripotent stem cell (UPPS) medium ${ }^{59}$.

PSCs play an important role for (comparative) developmental biology as well as for regenerative medicine. For these purposes, it is crucial to establish efficient protocols for the directed differentiation of NHP-PSCs into specific cell types. Here, we tested different protocols for cardiac differentiation, originally established for human PSCs. As for the cultivation of human and NHP-PSCs, we aimed for a universal cardiac differentiation protocol for better comparability between the primate species. For our rhesus and baboon iPSCs, we first tried to adopt an optimized chemically defined and growth factor-free cardiac differentiation protocol using a small molecule-based induction of differentiation ${ }^{35}$. This protocol is based on the modulation of Wnt activation and inhibition in a time-dependent manner as originally published by Lian and colleagues ${ }^{32} 33$. For cardiac induction, Lian et al., used RPMI 1640 supplemented with B27 without insulin, which contains the xenogeneic component BSA. In contrast, Burridge and colleagues developed for human cells a xeno-free and chemically less complex medium using RPMI 1640 supplemented only with L-ascorbic acid 2phosphate and rice-derived recombinant human albumin, tested on several human PSC lines with differentiation efficiencies up to $95 \%{ }^{35}$. However, none of our NHP-iPSC lines could be efficiently and reproducibly differentiated into cardiomyocytes applying both protocols using the Wnt activator Chir99021 and the inhibitors IWP-2 or IWR-1 alone. Notably, we observed an increased cell death of the NHP cells when using RPMI 1640 and Chir99021 only for mesoderm induction compared to RPMI 1640 supplemented with B27 without insulin and Chir99021. The growth factor-free cardiac differentiation protocols that work robustly for human PSCs. In contrast, the combination of Wnt signaling activation together with BMP4 and Activin A treatment followed by Wnt signaling inhibition was required to achieve efficient cardiac differentiation in our NHP-iPSCs.

Finally, we came up with a NHP cardiomyocyte differentiation protocol slightly modified compared to the protocol developed previously for human cells ${ }^{38}$. First beating NHP-iPSC-derived cardiomyocytes could be observed around day 8 of differentiation with higher differentiation efficiencies on day 12 in baboon (70\%) and human (72\%) compared to rhesus (53\%) cells (without prior selection). The fact that Wnt modulation using Chir99021 alone is not sufficient to differentiate the NHPiPSCs shows a clear difference between rhesus and baboon PSCs compared to human PSCs. There are only very few publications available showing directed cardiac differentiation protocols for NHP-PSCs to compare with our findings. Shiba and colleagues used the so-called matrix sandwich method, which also involves BMP4 and Activin A treatment, to differentiate cynomolgus macaque iPSC into cardiomyocytes 20212324 . However, their average differentiation efficiencies were relatively low (20\% cTNT positive cells before metabolic selection), and only one iPSC line was used in this study. Also, Zhao and colleagues reported only one rhesus iPSC line ${ }^{60}$. 


\section{Chapter 2: Reproducible Primate iPSC Generation, Cultivation, and Cardiac Differentiation under Chemically Defined Conditions}

Considering iPSC line heterogeneity, the overall value of these data might be limited. In order to provide general and robust data, we believe that it is crucial to demonstrate the feasibility of procedures with several independent cell lines from different individuals. Furthermore, to our knowledge, we report for the first time efficient directed cardiac differentiation of baboon iPSCs.

To ensure the functionality of the cardiomyocytes derived from UPPS-cultured iPSCs, we performed MEA analysis and generated EHMs ${ }^{38}$. Both assays showed that the cardiomyocytes respond to the non-selective $\beta 1$ - and $\beta 2$-adrenoreceptor agonist isoprenaline and that this effect can be reverted by propanolol. Also, the force of contraction of EHM generated from the primate iPSCs is $\mathrm{Ca} 2+$ concentrationdependent. EHMs are a powerful tool to study cardiomyocyte function and disease in vitro and may serve in customized formats as ATMPs for the regeneration of the degenerating human heart. To preclinically test this novel therapeutic approach in a meaningful way, it is important to do this in a coherent and clinical-equivalent NHP setting. In this context, the novel universal protocols allowing xeno-free and chemically defined PSC culture and cardiomyocyte differentiation represent an important step towards the translation of the experimental iPSC-based regeneration of the primate heart into the clinics.

\section{$5 \quad$ Acknowledgments (Chapter 2)}

We thank Nicole Umland, Ulrike Gödecke, Silke Günther, and Ilona Eggert for excellent technical assistance. We are grateful to Kerstin Zaft for administrative support. This work was supported by the German Center of Cardiovascular Research (DZHK Förderkennzeichen 81Z0300201) and the German Primate Center, which is financed as a Leibniz Institute by the Bundesrepublik Deutschland and the Bundesländer (Federal states). The plasmids pCXLE-hOCT3/4-shp53-F (\#27077), $p C X L E-h S K$ (\#27078) and $p C X L E-h U L$ (\#27080) for reprogramming were a kind gift from Shin'ya Yamanaka provided by addgene.

\section{Conflict of Interest (Chapter 2)}

The authors declare that they have no conflict of interest.

\section{$7 \quad$ References (Chapter 2)}

1. Phillips, K. A. et al. Why Primate Models Matter. Am. J. Primatol. 76, 801827 (2015).

2. Cardoso-Moreira, M. et al. Gene expression across mammalian organ development. Nature 571, 505-509 (2019). 
3. Carelli, F. N., Liechti, A., Halbert, J., Warnefors, M. \& Kaessmann, H. Repurposing of promoters and enhancers during mammalian evolution. Nat. Commun. 9, 1-11 (2018).

4. Harding, J. D. Nonhuman Primates and Translational Research : Progress , Opportunities , and Challenges. ILAR 58, 141-150 (2017).

5. Necsulea, A. \& Kaessmann, H. Evolutionary dynamics of coding and noncoding transcriptomes. Nat. Rev. 1, 1-15 (2014).

6. Rogers, J. \& Gibbs, R. A. Comparative primate genomics: emerging patterns of genome content and dynamics Jeffrey. Nat Rev Genet. 15, 347-359 (2014).

7. Zhang, Y. E. \& Long, M. New genes contribute to genetic and phenotypic novelties in human evolution. Curr Opin Genet Dev 29, 90-96 (2014).

8. Chellman, G. J. et al. Developmental and Reproductive Toxicology Studies in Nonhuman Primates. Birth Defects Res. 86, 446-462 (2009).

9. Faqi, A. S. A critical evaluation of developmental and reproductive toxicology in nonhuman primates. Syst. Biol. Reprod. Med. 58, 23-32 (2012).

10. Orsi, A. et al. Overview of the marmoset as a model in nonclinical development of pharmaceutical products. Regul. Toxicol. Pharmacol. 59, 19-27 (2011).

11. Brennan, F. R. et al. Safety testing of monoclonal antibodies in non-human primates : Case studies highlighting their impact on human risk assessment. MAbs 10, 1-17 (2018).

12. Buckley, L. A. et al. Considerations regarding nonhuman primate use in safety assessment of biopharmaceuticals. Int. J. Toxicol. 30, 583-590 (2011).

13. Li, T., Ai, Z. \& Ji, W. Primate stem cells : bridge the translation from basic research to clinic application. Sci China Life Sci. 61, 12-21 (2018).

14. Meyer, J. et al. Direct reprogramming of human neural stem cells by OCT4. Nature 461, 649-654 (2009).

15. Okita, K. et al. A more efficient method to generate integration-free human iPS cells. Nat. Methods 8, 409-412 (2011). 
16. Stadtfeld, M., Nagaya, M., Utikal, J., Weir, G. \& Hochedlinger, K. Induced pluripotent stem cells generated without viral integration. Science. 322, 945-949 (2008).

17. Warren, L. et al. Highly efficient reprogramming to pluripotency and directed differentiation of human cells with synthetic modified mRNA. Cell Stem Cell. 7, 618630 (2010).

18. Hanna, J. et al. Treatment of Sickle Cell Anemia Mouse Model with iPS Cells Generated from Autologous Skin. Science. 318, 1920-1923 (2007).

19. Kobayashi, Y. et al. Pre-Evaluated Safe Human iPSC-Derived Neural Stem Cells Promote Functional Recovery after Spinal Cord Injury in Common Marmoset without Tumorigenicity. PLoS One 7, 1-13 (2012).

20. Shiba, Y. et al. Allogeneic transplantation of iPS cell-derived cardiomyocytes regenerates primate hearts. Nature 538, 388-391 (2016).

21. Carey, B. W. et al. Reprogramming of murine and human somatic cells using a single polycistronic vector. PNAS 106, 157-162 (2009).

22. Biol, P. et al. Transplantation in the nonhuman primate MPTP model of Parkinson' s disease : update and perspectives. Primate Biol 4, 185-213 (2017).

23. Kikuchi, T. et al. Human iPS cell-derived dopaminergic neurons function in a primate Parkinson's disease model. Nature 548, 592-596 (2017).

24. Kriks, S. et al. Floor plate-derived dopamine neurons from hESCs efficiently engraft in animal models of PD. Nature 480, 547-551 (2012).

25. Vermilyea, S. C. \& Emborg, M. E. The role of nonhuman primate models in the development of cell- based therapies for Parkinson' s disease. J Neural Transm 125, 365-384 (2018).

26. Wang, S. et al. Autologous iPSC-derived dopamine neuron transplantation in a nonhuman primate Parkinson's disease model. Cell Discov. 1, 1-11 (2015).

27. Navara, C. S. et al. Derivation of induced pluripotent stem cells from the baboon: a nonhuman primate model for preclinical testing of stem cell therapies. Cell. Reprogram. 15, 495-502 (2013).

28. Wunderlich, S. et al. Primate iPS cells as tools for evolutionary analyses. Stem Cell Res. 12, 622-629 (2014). 


\section{Chapter 2: Reproducible Primate iPSC Generation, Cultivation, and Cardiac}

Differentiation under Chemically Defined Conditions

29. Debowski, K. et al. Non-viral generation of marmoset monkey iPS cells by a six-factor-in-one-vector approach. PLoS One 10, 1-21 (2015).

30. Tomioka, I. et al. Generating induced pluripotent stem cells from common marmoset ( Callithrix jacchus ) fetal liver cells using defined factors , including Lin28. Genes to Cells 15, 959-969 (2010).

31. Kahland, T. S. Modifying the common marmoset monkey (Callithrix jacchus) genome: transgenesis and targeted gene modification in vivo and in vitro. Biol. Fak. für Biol. und Psychol. Georg. Univ. Göttingen (Georg-August-Universität Göttingen). 0, 1-130 (2015).

32. Lian, X. et al. Robust cardiomyocyte differentiation from human pluripotent stem cells via temporal modulation of canonical Wnt signaling. PNAS 109, E1848E1857 (2012).

33. Lian, X. et al. Directed cardiomyocyte differentiation from human pluripotent stem cells by modulating Wnt/ $\beta$-catenin signaling under fully defined conditions Xiaojun. Nat Protoc 8, 162-175 (2013).

34. Thomson, J. A. et al. Isolation of a primate embryonic stem cell line. Proc. Natl. Acad. Sci. 92, 7844-8 (1995).

35. Cardiomyocytes, H. et al. Chemically Defined and Small Molecule-Based Generation of Human Cardiomyocytes. Nat Methods 11, 855-860 (2015).

36. Kattman, S. J. et al. Resource Stage-Specific Optimization of Activin / Nodal and BMP Signaling Promotes Cardiac Differentiation of Mouse and Human Pluripotent Stem Cell Lines. Stem Cell 8, 228-240 (2010).

37. Lian, X. et al. Chemically defined, albumin-free human cardiomyocyte generation. Nat Methods 12, 595-596 (2015).

38. Tiburcy, M. et al. Defined engineered human myocardium with advanced maturation for applications in heart failure modeling and repair. Circulation 135, 1832-1847 (2017).

39. Längin, M. et al. Consistent success in life-supporting porcine cardiac xenotransplantation. Nature 564, 430-433 (2018).

40. Liu, Y.-W. et al. Human ESC-Derived Cardiomyocytes Restore Function in Infarcted Hearts of Non-Human Primates. Nat Biotechnol. 36, 597-605 (2018). 


\section{Chapter 2: Reproducible Primate iPSC Generation, Cultivation, and Cardiac Differentiation under Chemically Defined Conditions}

41. Wolff, E., Suplicki, M. M. \& Behr, R. Primordial germ cells do not migrate along nerve fibres in marmoset monkey and mouse embryos. Reproduction 157, 101109 (2019).

42. Rodriguez-polo, I. et al. Baboon induced pluripotent stem cell generation by piggyBac transposition of reprogramming factors. Primate Biol., 6, 75-86 (2019).

43. Mandai, M. et al. .Autologous Induced Stem-Cell-Derived Retinal Cells for Macular Degeneration . N. Engl. J. Med. 376, 1038-1046 (2017).

44. Wu, Y. et al. Nonhuman Primate Induced Pluripotent Stem Cells in Regenerative Medicine. Stem Cells Int. 2012, 1-7 (2012).

45. Deleidi, M., Hargus, G., Hallett, P., Osborn, T. \& Isacson, O. Development of histocompatible primate induced pluripotent stem cells for neural transplantation. Stem Cells. 29, 1052-1063 (2011).

46. Liu, H. et al. Generation of Induced Pluripotent Stem Cells from Adult Rhesus Monkey Fibroblasts. Cell Stem Cell 3, 587-590 (2008).

47. N.Marchetto, M. C. et al. Differential L1 regulation in pluripotent stem cells of humans and apes. Nature 503, 1-8 (2014).

48. Yuehong Wu, Zhang, Y., Mishra, A., Tardif, S. D. \& Hornsby, P. J. Generation of induced pluripotent stem cells from newborn marmoset skin fibroblasts. Stem Cell Res 4, 180-188 (2011).

49. Zhong, B. et al. Efficient Generation of Nonhuman Primate Induced Pluripotent Stem Cells. Stem Cells Dev. 20, (2011).

50. Hong, S. G. et al. Path to the clinic: Assessment of iPSC-based cell therapies in vivo in a non-human primate model. Cell Rep 7, 1298-1309 (2014).

51. Yada, R. C. et al. Rhesus Macaque iPSC Generation and Maintenance. Curr Protoc Stem Cell Biol. 41, 1-17 (2018).

52. Weltner, J., Anisimov, A., Alitalo, K., Otonkoski, T. \& Trokovica, R. Induced Pluripotent Stem Cell Clones Reprogrammed via Recombinant Adeno-Associated Virus-Mediated Transduction Contain Integrated Vector Sequences. J. Virol. 86, 4463-4467 (2012).

53. Grow, D. A. et al. Differentiation and Characterization of Dopaminergic Neurons From Baboon Induced Pluripotent Stem Cells. Stem Cells Transl. Med. 5, 1133-1144 (2016). 
54. Navara, C. S., Chaudhari, S. \& McCarrey, J. R. Optimization of culture conditions for the derivation and propagation of baboon (Papio anubis) induced pluripotent stem cells. PLoS One 13, 1-16 (2018).

55. Zhang, X., Cao, H., Bai, S., Huo, W. \& Ma, Y. Differentiation and characterization of rhesus monkey atrial and ventricular cardiomyocytes from induced pluripotent stem cells. Stem Cell Res. 20, 21-29 (2017).

56. Chen, G. et al. Chemically defined conditions for human iPS cell derivation and culture. Nat Methods 8, 424-429 (2011).

57. $\mathrm{Wu}, \mathrm{J}$. et al. An alternative pluripotent state confers interspecies chimaeric competency. Nature 000, 1-23 (2015).

58. Qi, X. et al. BMP4 supports self-renewal of embryonic stem cells by inhibiting mitogen-activated protein kinase pathways. PNAS 101, 6027-6032 (2004).

59. Kim, H. et al. Modulation of $\beta$-catenin function maintains mouse epiblast stem cell and human embryonic stem cell self-renewal. Nat Commun 4, 1-21 (2014).

60. Zhao, X. et al. Comparison of Non-human Primate versus Human Induced Pluripotent Stem Cell-Derived Cardiomyocytes for Treatment of Myocardial Infarction. Stem Cell Reports 10, 422-435 (2018).

\section{Supplementary material (Chapter 2)}

\subsection{Tables}

\begin{tabular}{cccc}
\hline & Company & Cat.-No. & Dilution \\
\hline AFP & Dako & A0008 & 1 to 100 \\
$\alpha$-actinin & Sigma-Aldrich & A7811 & 1 to 1000 \\
$\begin{array}{c}\beta \text {-tubulin } \\
\text { III }\end{array}$ & Sigma-Aldrich & T8660 & 1 to 1000 \\
Cx43 & Abcam & ab11370 & 1 to 1000 \\
cTNI & Abcam & ab47003 & 1 to 200 \\
cTNT & Miltenyi Biotec & $130-106-687$ & 1 to 10 \\
cTNT & Thermo Fisher & MS295PABX & 1 to 200 \\
LIN28 & R\&D Systems & AF3757 & 1 to 300 \\
MLC2a & Synaptic & S11-011 & 1 to 200 \\
\hline
\end{tabular}


Chapter 2: Reproducible Primate iPSC Generation, Cultivation, and Cardiac Differentiation under Chemically Defined Conditions

\begin{tabular}{cccc} 
NANOG & Cell Signalling & 4903 & 1 to 400 \\
SALL4 & Abcam & ab57577 & 1 to 200 \\
\hline SMA & Sigma-Aldrich & A2547 & 1 to 1000 \\
SOX9 & Merck & AB5535 & 1 to 1000 \\
SSEA4 & Abcam & ab16287 & 1 to 200 \\
Titin & Merck & MAB1553 & 1 to 50 \\
TRA-1-60 & Abcam & ab16288 & 1 to 200 \\
\hline
\end{tabular}

Suppl. Table 1: Primary antibodies used in this study.

\begin{tabular}{|c|c|c|c|}
\hline Name & Company & $\begin{array}{l}\text { Cat.- } \\
\text { No. } \\
\end{array}$ & Dilution \\
\hline Alexa555-goat- $\alpha$-mouse IgG & Thermo Fisher & A21424 & $\begin{array}{l}1 \text { to } \\
1000\end{array}$ \\
\hline Alexa488-goat- $\alpha$-mouse IgG & Thermo Fisher & A11029 & $\begin{array}{l}1 \text { to } \\
1000\end{array}$ \\
\hline Alexa488-goat- $\alpha$-mouse $\operatorname{IgG} / \operatorname{IgM}$ & Thermo Fisher & A10680 & $\begin{array}{l}1 \text { to } \\
1000\end{array}$ \\
\hline Alexa488-donkey- $\alpha$-goat IgG & Thermo Fisher & A11055 & $\begin{array}{l}1 \text { to } \\
1000\end{array}$ \\
\hline Alexa488-donkey- $\alpha$-rabbit IgG & Thermo Fisher & A21206 & $\begin{array}{l}1 \text { to } \\
1000\end{array}$ \\
\hline
\end{tabular}

Suppl. Table 2: Secondary antibodies used in this study. 


\begin{tabular}{|c|c|c|c|c|}
\hline \multicolumn{5}{|c|}{ Plasmid detection in genomic DNA } \\
\hline Name & Sequence & $\mathrm{F}[\mathrm{bp}]$ & $\mathrm{TM}\left[{ }^{\circ} \mathrm{C}\right]$ & $\mathrm{C}$ \\
\hline \multirow{2}{*}{ WPRE } & for: 5'- GCT ATT GCT TCC CGT ATGGC - $3^{\prime}$ & \multirow{2}{*}{470} & \multirow{2}{*}{54} & \multirow{2}{*}{32} \\
\hline & rev: 5'- CAA AGG GAG ATC CGA CTC GT -3' & & & \\
\hline \multirow{2}{*}{ EBNA-LoxP (2) } & for: 5'- AAG AGG AGGGGT CCC GAGA -3' & \multirow{2}{*}{555} & \multirow{2}{*}{61} & \multirow{2}{*}{32} \\
\hline & rev: 5'- GCC AAT GCA ACT TGG ACGTT -3' & & & \\
\hline \multirow{2}{*}{ OriP1 } & for: 5'- GGT TCA CTA CCC TCG TGG AAT -3' & \multirow{2}{*}{592} & \multirow{2}{*}{57} & \multirow{2}{*}{32} \\
\hline & rev: 5'-CGG GGC AGT GCA TGT AAT -3' & & & \\
\hline \multirow{2}{*}{ OriP2 } & for: 5'- GGT GAC TGT GTGCAGCTT TG-3' & \multirow{2}{*}{416} & \multirow{2}{*}{54} & \multirow{2}{*}{32} \\
\hline & rev: 5'- GGA GCT GAGTGA CGT GAC AA -3' & & & \\
\hline \multirow{2}{*}{$\beta$-actin } & for: 5'- GAC CTG ACT GAC TAC CTC ATG -3' & \multirow{2}{*}{$379 / 380$} & \multirow{2}{*}{61} & \multirow{2}{*}{32} \\
\hline & rev: 5'- GGT AGT TTC GTG GAT GCC ACA -3' & & & \\
\hline \multicolumn{5}{|c|}{ Endogenous (endo) and exogenous (exo) pluripotency marker expression (RT-PCR) } \\
\hline Name & Sequence & $\mathrm{F}[\mathrm{bp}]$ & $\mathrm{TH}\left[{ }^{\circ} \mathrm{C}\right]$ & \# \\
\hline \multirow{2}{*}{ KLF4 (exo) } & for: 5'- TTC ATC GAC GAGGCT AAGCG-3' & \multirow{2}{*}{812} & \multirow{2}{*}{53} & \multirow{2}{*}{30} \\
\hline & rev: 5'- TCA CTGACA GCC ATGGTGAA - $3^{\prime}$ & & & \\
\hline \multirow{2}{*}{ OCT4 (exo) } & for: 5'- TGA TCC TCG GAC CTGGCT AA -3' & \multirow{2}{*}{1021} & \multirow{2}{*}{54} & \multirow{2}{*}{30} \\
\hline & rev: 5'- TCCCCGAAGCTTGAATTCGC - $3^{\prime}$ & & & \\
\hline \multirow{2}{*}{ OCT4 (endo) } & for: 5'- GAGAAGGAGAAGCTG GAGCAA -3' & \multirow{2}{*}{841} & 53 & 30 \\
\hline & rev: $5^{\prime}-$ ACA TCC TTC TCG AGC CCA A -3' & & & \\
\hline LINר28 (مu) & for: 5'- ACT CAA ACT GGC TGG GGA TG -3' & 327 & $51+>$ & 30 \\
\hline LIN 28 (eXo) & rev: 5'- TTC AAGCTC CGG AAC CCT TC - $3^{\prime}$ & & & \\
\hline & for: 5'- GGG TGT TCT GTA TTGGGA GTG -3' & 271 & & \\
\hline LIN28 (endo) & rev: 5'- GCA CCC TAT TCC CAC TTT CTC -3' & 371 & 61 & 30 \\
\hline NANG & for: 5'- CAG AGA TAC CTC AGC CTC CAG -3' & 562 & $54+2$ & \\
\hline NANUG & rev: 5' - CTT CAGGTT GCA TGT TCGT - $3^{\prime}$ & 502 & 54 & 30 \\
\hline $\operatorname{sox} 2$ & for: 5'- GGT AGG AGC TTT GCA GGA AGT -3' & 428 & 61 & \\
\hline & rev: 5'-CCA ACG ATGTCA ACC TGC ATG-3' & & & \\
\hline & for: $5^{\prime}$ - TGG ATG ATG ATA TCG CCG CGC T -3' & 324 & & \\
\hline & rev: 5'-GGGCCT CGGTCA GCA GCA CGG-3' & & & \\
\hline
\end{tabular}

Suppl. Table 3. Oligonucleotides for PCR analyses. F: amplicon length; $\mathrm{T}_{\mathrm{M}}$ : annealing temperature; C: cycles 


\begin{tabular}{|c|c|c|c|c|c|c|c|c|c|c|}
\hline & Protein & & & & & & & & & \\
\hline & $\begin{array}{l}\text { hKLF } \\
4\end{array}$ & $\begin{array}{l}\text { rKLF } \\
4\end{array}$ & $\begin{array}{l}\text { hMY } \\
\text { C }\end{array}$ & $\begin{array}{l}\text { rMY } \\
\text { C }\end{array}$ & $\begin{array}{l}\text { hSOX } \\
2\end{array}$ & $\begin{array}{l}\text { rSOX } \\
2\end{array}$ & $\begin{array}{l}\text { hOCT } \\
4\end{array}$ & $\begin{array}{l}\text { rOCT } \\
4\end{array}$ & $\begin{array}{l}\text { hLIN2 } \\
8\end{array}$ & $\begin{array}{l}\text { rLIN2 } \\
8\end{array}$ \\
\hline $\begin{array}{l}\text { hKLF } \\
4\end{array}$ & 100 & 99,37 & & & & & & & & \\
\hline $\begin{array}{l}\text { rKLF } \\
4\end{array}$ & 99,37 & 100 & & & & & & & & \\
\hline hMYC & & & 100 & 98,46 & & & & & & \\
\hline rMYC & & & 98,46 & 100 & & & & & & \\
\hline hSOX2 & & & & & 100 & 100 & & & & \\
\hline rSOX2 & & & & & 99,37 & 100 & & & & \\
\hline $\begin{array}{l}\text { hOCT } \\
4\end{array}$ & & & & & & & 100 & 99,17 & & \\
\hline $\begin{array}{l}\text { rOCT } \\
4\end{array}$ & & & & & & & 99,17 & 100 & & \\
\hline hLIN28 & & & & & & & & & 100 & 99,04 \\
\hline rLIN28 & & & & & & & & & 99,04 & 100 \\
\hline
\end{tabular}

Suppl. Table 4: Protein similarity analysis for both human and rhesus pluripotency genes compared via SerialCloner Alignment. All values are given in percent.

\section{Unsuccessful growth factor-free NHP cardiomyocyte differentiation protocols}

Approach 1: When reaching confluency of about 80-90\%, the UPPS medium was replaced by mesodermal induction medium (Roswell Park Memorial Institute Medium (RPMI 1640, Thermo Fisher), $0.2 \mathrm{mg} / \mathrm{ml}$ L-ascorbic acid 2-phosphate (SigmaAldrich), $0.5 \mathrm{mg} / \mathrm{ml}$ recombinant human albumin (Sigma-Aldrich), with different concentrations of Chir99021 (1, 2.5, 4, and $5 \mu \mathrm{M}))$. After 48h, medium was replaced by cardiac induction medium (RPMI 1640, $0.2 \mathrm{mg} / \mathrm{ml} \mathrm{L}$-ascorbic acid 2-phosphate, $0.5 \mathrm{mg} / \mathrm{ml}$ recombinant human albumin, $5 \mu \mathrm{M}$ IWP-2 (Millipore) or IWR-1. At day 4 and 6, medium was changed with RPMI 1640, $0.2 \mathrm{mg} / \mathrm{ml} \mathrm{L}$-ascorbic acid 2-phosphate, and $0.5 \mathrm{mg} / \mathrm{ml}$ recombinant human albumin. From day 8 on, medium was replaced by cardiomyocyte cultivation medium (RPMI 1640, B27 with insulin (Thermo Fisher)) and changed every 2-3 days. A similar protocol was tested with a slightly modified mesodermal induction medium using RPMI 1640 with Chir99021 only.

Approach 2: A second cardiac differentiation protocol used RPMI 1640 and B27 without insulin. At a confluency of about $80-90 \%$, the UPPS medium was replaced by mesodermal induction medium (RPMI 1640, B27 without insulin (Thermo Fisher), with different concentrations of Chir99021 tested (1, 3, 4, 8, 9 and $10 \mu \mathrm{M})$ ). After 24h, medium was changed with RPMI 1640, B27 without insulin. At day 3, medium was replaced by cardiac induction medium (RPMI 1640, B27 without insulin (half fresh, half left), $5 \mu \mathrm{M}$ IWP-2 or IWR-1). At day 5, medium was changed with RPMI 1640, B27 without insulin. From day 7 on, medium was replaced by cardiomyocyte 
cultivation medium (RPMI 1640, B27 with insulin) and changed every 2-3 days. A similar protocol was tested using IMDM instead of RPMI 1640.

\begin{tabular}{cll}
\hline Condition\# & Cultivation media & Additives \\
\hline 1 & Essential 8 medium & - \\
2 & Essential 8 medium & $2.5 \mu \mathrm{M}$ IWR-1 \\
3 & Essential 8 medium & $2.5 \mu \mathrm{M}$ IWR-1 \\
& & $25 \mathrm{ng} / \mathrm{ml}$ BMP4 \\
4 & Essential 8 medium & $2.5 \mu \mathrm{M}$ IWR-1 \\
& & $3 \mu \mathrm{M}$ CHIR99021 \\
5 & StemMACS iPS-Brew XF & - \\
6 & StemMACS iPS-Brew XF & $2.5 \mu \mathrm{M}$ IWR-1 \\
7 & StemMACS iPS-Brew XF & $2.5 \mu \mathrm{M}$ IWR-1 \\
& & $25 \mathrm{ng} / \mathrm{ml}$ BMP4 \\
& & $2.5 \mu \mathrm{M}$ IWR-1 \\
& & $3 \mu \mathrm{M}$ CHIR99021 \\
& StemMACS iPS-Brew XF & $1 \mu \mathrm{M}$ IWR-1 \\
& & $0.5 \mu \mathrm{M}$ CHIR99021
\end{tabular}

Suppl. Table 5. Tested conditions to optimize a standardized cultivation method for human and NHPPSCs.

\subsection{Supplementary figures}



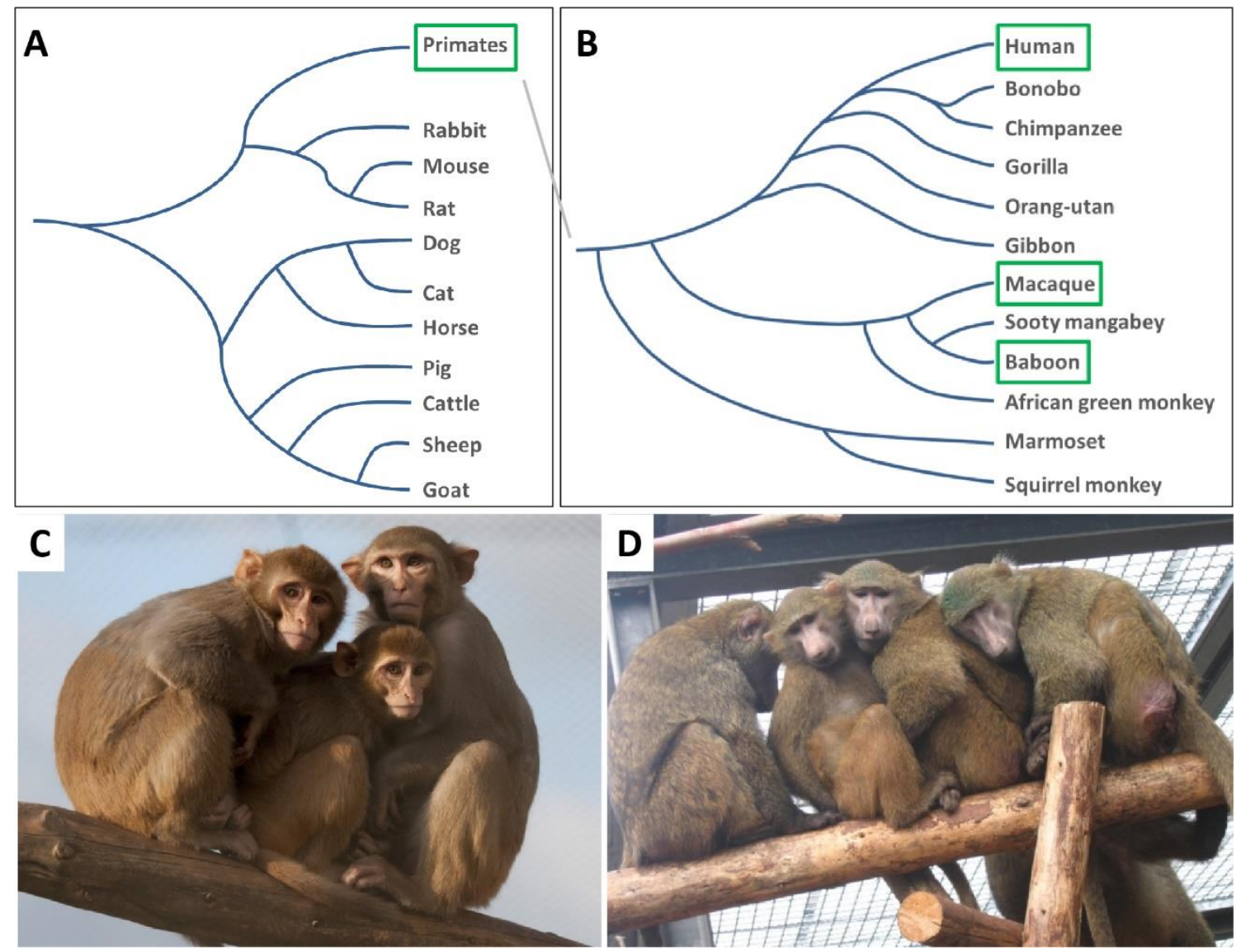

Figure S1. Non-human primates in basic and translational research. (A) Phylogenetic tree of mammals used as model organisms in research (after Hedges 2002), (B) phylogenetic tree of selected primates (after Rogers \& Gibbs $2014{ }^{6}$ ), (C) rhesus macaques, (D) olive baboons. Photographic images by German Primate Center (DPZ). 


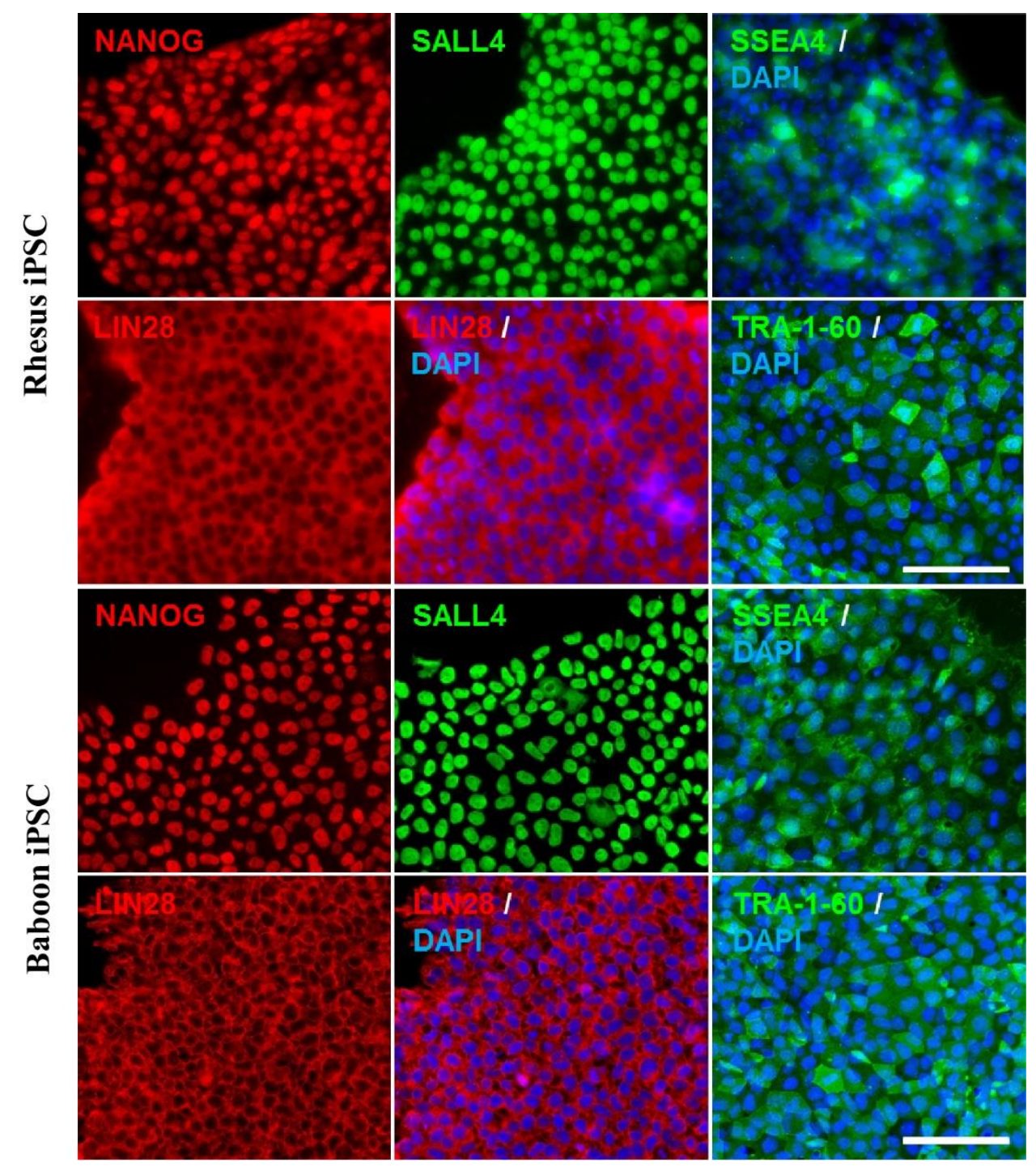

Figure S2. Pluripotency gene expression in NHP iPSCs. Rhesus (upper panel) and baboon (lower panel) iPSCs express pluripotency related transcription factors NANOG and SALL4, pluripotency related surface markers SSEA4 and TRA-1-60as well as LIN28. Scale bar $=100 \mu \mathrm{m}$. 


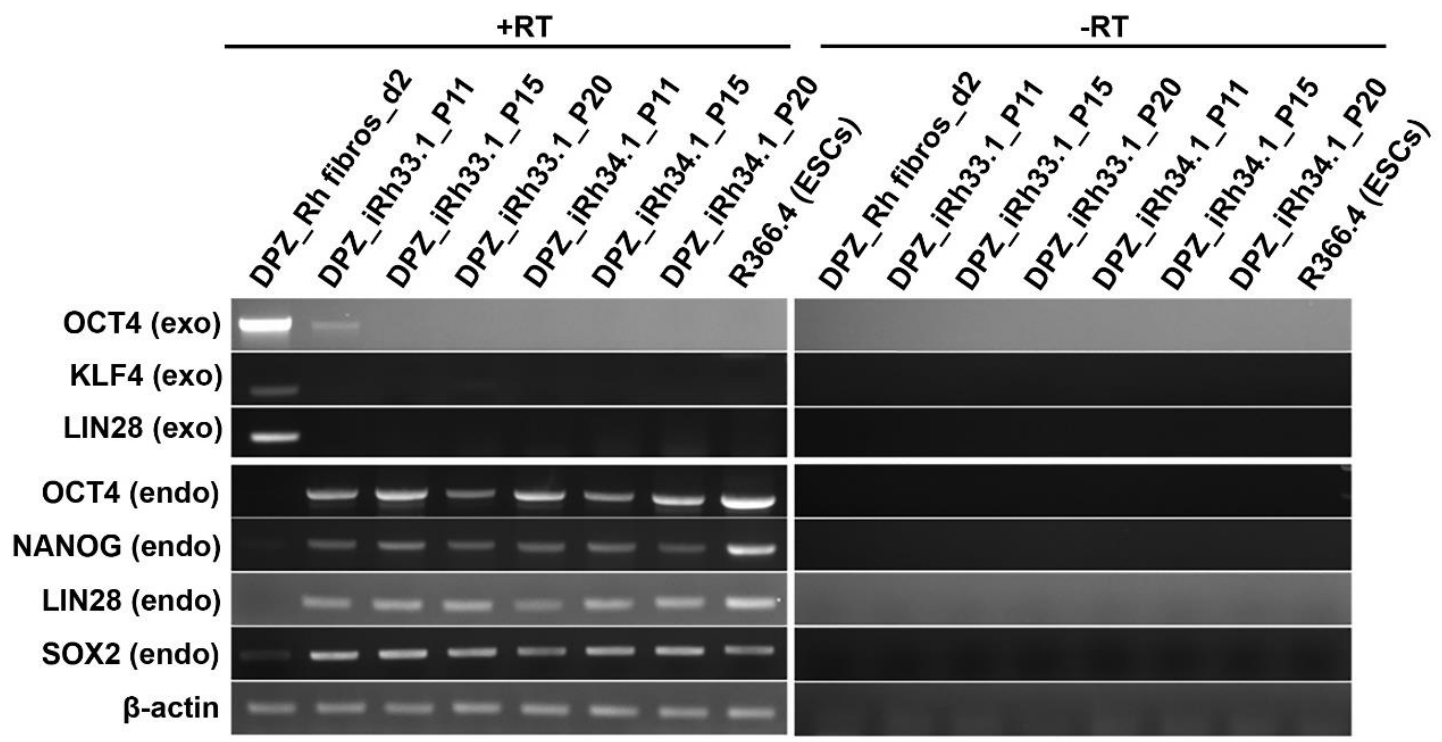

Figure S3. Time-dependent exogenous (exo) and endogenous (endo) pluripotency-related gene expression in two generated rhesus iPSC lines. The exogenous (plasmid regulated) gene expression of OCT4, KLF4 and LIN28 is detectable in rhesus fibroblasts two days after nucleofection. OCT4 (exo) expression is still detectable in iPSC line Rh33.1 at passage 11 but disappears in later passages. In cell line iRh34.1 exogenous gene expression is not detectable at passage 11 or higher. The pluripotencyrelated endogenous gene expression is detectable in both iPSC cell lines at passage 11 and higher as well as in the rhesus ESC line Rh366.4, but not in fibroblasts two days after nucleofection. The left panel shows results after PCR with prior reverse transcription $(+\mathrm{RT})$ and on the right without reverse transcription (-RT). 
(A)

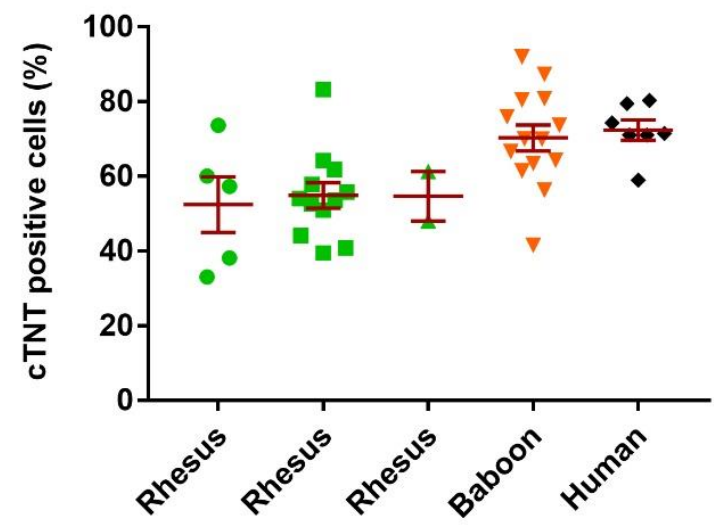

- DPZ_iRh24.4 (n=5)

- DPZ_iRh33.1 $(\mathrm{n}=12)$

- DPZ_iRh34.1 (n=2)

$\checkmark \quad D P Z$ iPv2.8 $(n=14)$

- DPZ_iHu1.2 (n=7)

(B)

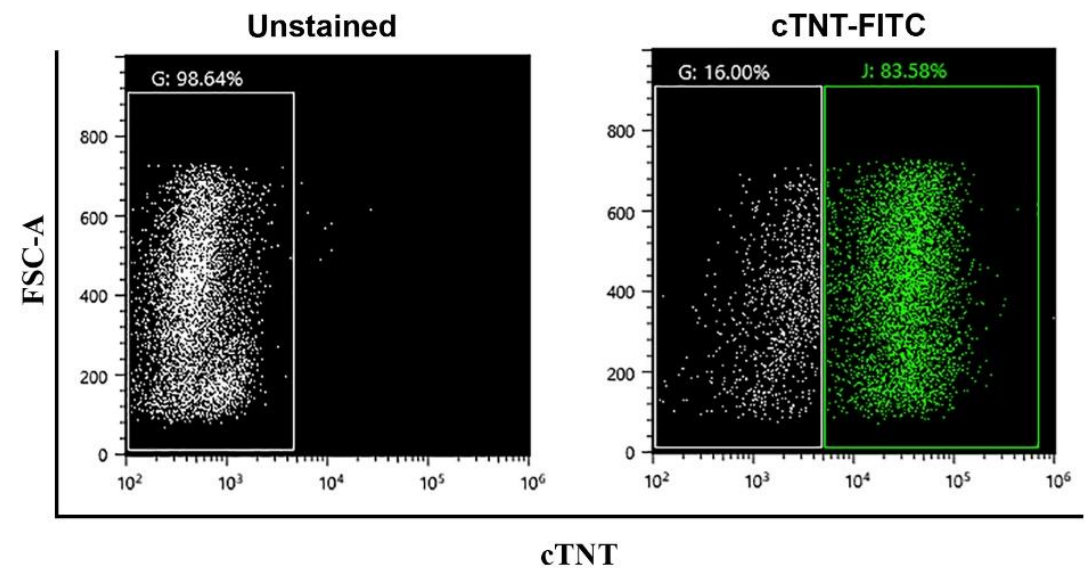

Figure S4. (A-B) Differentiation efficiencies of the human and NHP-iPSCs were defined by cardiac troponin $\mathrm{T}$ positive cells at day 12 of differentiation by flow cytometry (before metabolic selection). (A) Quantitative analysis of differentiation efficiency. (B) Representative gating used for the sorting. 


\section{Chapter 3: A piggyBac-based platform for genome editing and clonal rhesus macaque iPSC line derivation}

\section{Author contribution statement}

IRP, SM, and RB conceived and designed the experiments. IRP, SM, SP, AM, IG, YT, and DU performed the experiments. IRP, SM, SP, AM, IG, YT, DU, FM, and RB analyzed the data. RD and IB designed and performed the teratoma assay and karyotyping, respectively. AM and FM performed the methylation analysis. RB and IRP wrote the paper with contributions from all coauthors.

\begin{tabular}{|l|l|}
\hline \multicolumn{2}{|c|}{$\begin{array}{l}\text { Author contribution statement } \\
\text { generation and genome editing } \\
\text { Chapter 3: Piggybac system as a versatile tool for rhesus macaque iPS generation and genome } \\
\text { editing }\end{array}$} \\
\hline Figure 1 & IRP and SM performed the reprogramming of the different lines \\
\hline Figure 1, Suppl. Figure 6 & IRP performed the adaptation to feeder-free and AP staining \\
\hline Figure 2, Suppl. Figure 1, 2 & IRP performed iPSC characterization \\
\hline $\begin{array}{l}\text { Figure 2 Suppl. Figure 3, 4, } \\
\text { 6 }\end{array}$ & $\begin{array}{l}\text { RD performed the teratoma formation assay } \\
\text { IRP, SM, RB, and RD analyzed the teratoma histology }\end{array}$ \\
\hline Figure 3, Suppl. Figure 5 & FM, AM, TH performed the methylation experiments and analysis \\
\hline Figure 4 & IRP performed the transposon removal \\
\hline Figure 5 and 6 & IRP, YT, IG, and DU (All under master lab rotation or master thesis \\
& supervised by IRP) performed the single-cell cloning experiments \\
\hline Figure 6 & SP construct the vector $P T T$-PB- $p$ CAG- $C$ Cas9-GFP-U6-gRNA-Neo \\
\hline Table 1 & IRP performed the similarity analysis \\
\hline Suppl. Figure 7 & IRP generated the isogenic controls \\
\hline Experiment design & IRP, SM, and RB \\
\hline Manuscript writing & IRP and RB, with the contribution of all authors \\
\hline
\end{tabular}




\section{A piggyBac-based platform for genome editing and clonal rhesus macaque iPSC line derivation}

Ignacio Rodriguez-Polo ${ }^{1,2}$, Sophie Mißbach ${ }^{1,2}$, Stoyan Petkov ${ }^{1,2}$, Felix Mattern ${ }^{3}$, Anna Maierhofer $^{3}$, Iga Grządzielewska ${ }^{1,4}$, Yuliia Tereshchenko ${ }^{1,4}$, Daniel Urrutia-Cabrera ${ }^{1}$

\#, Thomas Haaf ${ }^{3}$, Ralf Dressel ${ }^{2,5}$, Iris Bartels ${ }^{6}$, Rüdiger Behr ${ }^{1,2 *}$

${ }^{1}$ Research Platform Degenerative Diseases, German Primate Center - Leibniz Institute for Primate Research, Kellnerweg 4, 37077 Göttingen, Germany

${ }^{2}$ German Center for Cardiovascular Research (DZHK), Partner site, Göttingen, Germany

${ }^{3}$ Institut für Humangenetik, Universität Würzburg, Biozentrum, Am Hubland, 97074 Würzburg

${ }^{4}$ Max Planck molecular Biology program (M.Sc./Ph.D.), Justus-von-Liebig-Weg 11, 37077 Göttingen, Germany

${ }^{5}$ Institute of Cellular and Molecular Immunology, University Medical Center Göttingen, Humboldtalle 34, 37073 Göttingen, Germany

${ }^{6}$ Institute of Human Genetics, University Medical Center Göttingen, Robert-Koch-Str. 40, 37075 Göttingen, Germany

${ }^{\#}$ Current address: Cellular reprogramming unit, Center for eye research Australia, 75 Commercial Road, Melbourne 3004, Australia

*Correspondence:

Rüdiger Behr

Research Platform Degenerative Diseases

German Primate Center - Leibniz Institute for Primate Research

Kellnerweg 4, 37077 Göttingen, Germany

Fax: 0049-(0)551-3851431

Email: rbehr@dpz.eu 
Abstract. Non-human primates (NHP) are, due to their close phylogenetic relationship with humans, excellent animal models to study clinically relevant human mutations. However, the toolbox for the genetic modification of NHP is less developed so far than that for the genetic manipulation of other species, e.g., mice. Therefore, it is necessary to further develop and refine genome editing approaches in NHP. NHP pluripotent stem cells (PSC) share key molecular signatures with the early embryo, which is an important target for genomic modification. Therefore PSC are a valuable test system for the validation of embryonic genome editing approaches. In the present study, we made use of the versatility of the piggyBac system for different purposes in NHP biotechnology and genome editing. These include 1) Reprogramming of rhesus macaque fibroblasts to induced pluripotent stem cells (iPSCs); 2) Removal of the transgene resulting in transgene-free iPSCs, that could be cultured under feeder-free conditions; 3) Development of an approach to edit the genome of rhesus macaque PSCs with high efficiency; 4) Establishment of a novel protocol for the derivation of gene-edited monoclonal iPSC lines. These findings facilitate efficient testing of genome editing approaches in NHP before in vivo application.

\section{Introduction}

Cardiovascular and neurodegenerative diseases are the primary causes of death worldwide ${ }^{12}$. Even though these disorders have a complex etiology they are often of genetic origin ${ }^{34}$. To further understand the development and progression of these diseases, highly predictive model systems need to be established. Besides cell and organoid models, it is necessary to use animal models that share the complexity of the human nervous, immune, and cardiovascular systems ${ }^{56}$. Non-human primates (NHP) as our evolutionary closest relatives are excellent animal models, as reflected by high genetic, physiological, developmental, and metabolic resemblance with humans ${ }^{6-10}$.

In comparison with other model organisms, NHP biomedical models are less established ${ }^{1011}$, but the demand for NHP models in biomedicine is strongly increasing. Due to the difficulties associated with genetic modifications of NHP, most studies mimicking human diseases use drug or surgically induced conditions 5111213 . However, in the last years, the emergence of new genome editing-tools like CRISPR-Cas9 has revolutionized the generation of genetically modified animals ${ }^{14}$. The first NHP species that were genetically modified, at that time by classical lentiviral transgenesis, were the marmoset and the rhesus macaque 91315161718 . However, the genetic modification of NHP entails difficulties not found in other model species. Usually, NHP have smaller litter size and long gestation periods. In consequence, it is challenging and cost-intensive to obtain a high number of embryos ${ }^{71311}$. Furthermore, the injection of (genetically modified) NHP-PSCs into primate embryos has not been successful so far, in contrast to mouse. Many efforts have been made to overcome these difficulties by refining and adapting protocols developed for mouse, rat, or bovine to NHP ${ }^{9}$. Finally, the generation of transgenic NHP is also ethically controversial ${ }^{10}{ }^{19}$. All of these obstacles together make the careful evaluation of genome editing tools necessary 
before their application in vivo ${ }^{20}$. In order to search for an appropriate genome editing approach, certain aspects must be critically assessed: (1) efficacy and efficiency of the system, (2) translatability of the generated data, and (3) safety of the NHP during the experiment. To address these issues, species-specific pluripotent stem cells are a valuable tool for the in vitro validation of the efficiency and accuracy of the respective editing approaches.

NHP-PSC, i.e., embryonic stem cells (ESCs) and iPSCs, share many features with the pluripotent cells of the early embryo ${ }^{21}{ }^{22}$. They can be used to study the efficacy of genome editing systems and predict, upon directed differentiation into specific cell types, potential phenotypic alterations that might be observed later on in the animal. Using this workflow, it is possible to evaluate both at the same time, the potential risks for the animals as well as the feasibility of the in vivo model.

For DNA based reprogramming and genome editing, it is crucial to deliver relatively large constructs with high efficiency. Furthermore, robust long-term expression can be relevant for certain purposes, specifically for reprogramming of differentiated cells to iPSCs. This requires the exogenous expression of key reprogramming factors to awake pluripotency in somatic cells ${ }^{23} 24$. In the case of genome editing, it is necessary to express nucleases plus guide RNAs ${ }^{24} 25$. The piggyBac transposon is a mobile genetic element originally identified in a moth (Trichoplusia $\mathrm{ni}$ ) that efficiently transposes between the donor vector and host chromosomes. This system has two major advantages, (1) it has almost no cargo limit, and (2) it is fully reversible, leaving no footprint in the genome after excision ${ }^{24}{ }^{26}$. The piggyBac transposon/transposase system consists of a transposase that recognizes piggyBac-specific inverted terminal repeat sequences (ITRs) located on both sides of the transposon cassette. The transposase excises the transposable element to integrate it into TT/AA chromosomal sites. Together, these characteristics make the piggyBac an excellent biotechnological tool for exploring the genetic modification of NHP 23262728 .

Here we show and characterize two main applications of the piggyBac system, which will facilitate the evaluation of genome editing in vitro before in vivo applications. First, the previously published piggyBac 6-reprogramming factor construct can efficiently generate iPSC from adult rhesus macaque fibroblasts. Moreover, the generated iPSCs can be cultured in feeder-free conditions. We demonstrate that the exogenous expression of the reprogramming construct gets silenced during reprogramming and passaging. To ensure the stability of the reprogrammed state, we removed the reprogramming cassette from the iPSC after reprogramming, thereby generating transgene-free macaque iPSC. Secondly, we employed the piggyBac vector for highly efficient genome editing. Finally, we developed a robust protocol for clonal derivation of rhesus macaque pluripotent stem cells (PSC). Combining our tools and protocols, we succeeded in the straight forward establishment of clonal rhesus monkey iPSC lines harboring clinically relevant mutations in Titin (TTN) gene. 


\section{Results}

\subsection{PiggyBac 6-factors in one vector system can reprogram adult rhesus macaque skin fibroblast.}

Four independent rhesus iPSC lines, named DPZ_iRhpb\#1-4, were generated via piggyBac transposition using our previously published six factors in one vector transposon system (Fig. 1, A) ${ }^{23}$. The reprogramming transposon encodes the marmoset factors SOX2, OCT4A, KLF4, c-MYC, NANOG, and LIN28. Marmoset and rhesus macaque pluripotency factors showed a very high degree of conservation on the cDNA and the protein level (Table 1). The iPSC lines were generated from skin fibroblast from 2 adult macaques (DPZ_iRhpb\#1-3, male) (DPZ_iRhpb\#4, female). Approximately 20 days after transfection and selection of the fibroblasts, the first colonies emerged; new colonies appeared at least until day 60. Between 100 and 150 colonies were identified per reprogramming experiment. Primary colonies showed the typical morphology of human iPSCs in feeder cell culture. During the first passages, the best colonies were selected by manual picking according to their morphology. Four of the colonies were selected in passage 5 to proceed with further passaging and characterization under feeder cell conditions (Fig. 1, A). In passage 10 to 20, the lines became stable, showing almost no differentiation. First assessment of the pluripotent state of the cells was done by testing alkaline phosphatase activity (AP). All generated lines showed alkaline phosphatase activity (Fig. 1, B, C).

(A)

(B)
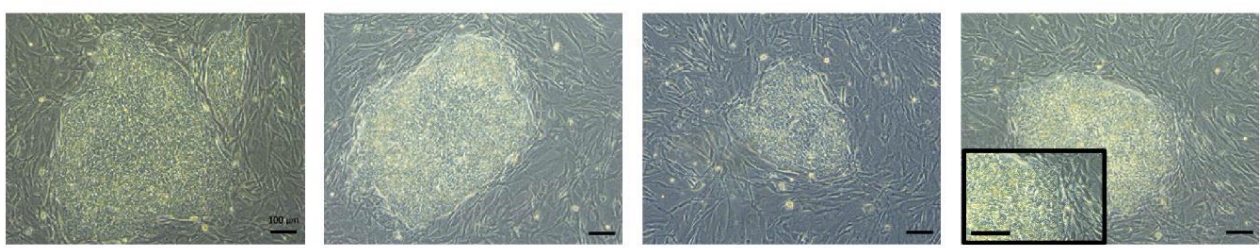

(C)
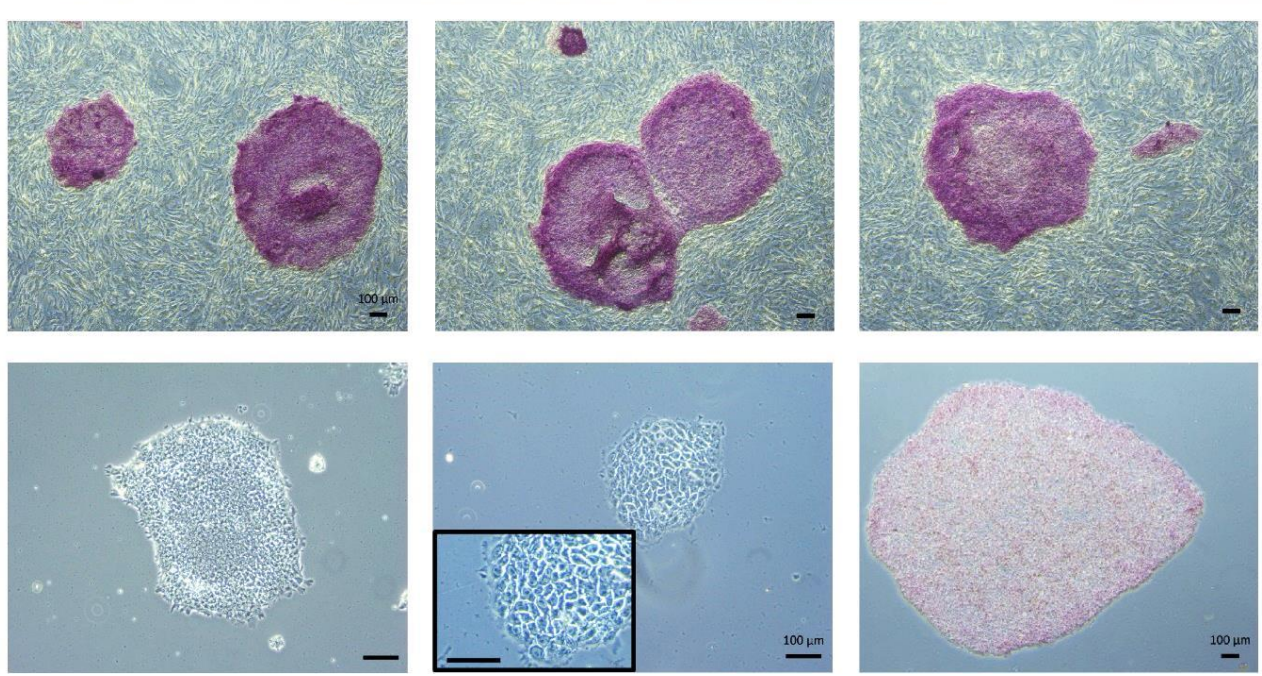


\section{Chapter 3: A piggyBac-based platform for genome editing and clonal rhesus macaque iPSC line derivation}

Figure 1: Rhesus induced pluripotent stem cells generated by piggyBac transposition. (A) Rhesus iPSC morphology on feeders (left to right DPZ_iRhpb\#1-4), (B) Alkaline phosphatase staining (left to right DPZ_iRhpb\#1-3), (C) PSC adaptation to feeder-free culture conditions. Left to right bright field pictures Rh366.4 ESCs, DPZ_iRhpb\#4, and alkaline phosphatase staining of DPZ_iRhpb\#4. Scale bars $100 \mu \mathrm{m}$.

Three cell lines, DPZ_iRhpb\#2, DPZ_iRhpb\#4, and rhesus ESC (Rh_ESC) were adapted to feeder-free conditions (DPZ_iRhpb\#4 and Rh_ESC, Fig. 1, C) (DPZ_iRhpb\#2, data not shown) using Stem Max iPS-Brew supplemented with $1 \mu \mathrm{M}$ IWRI and 0,5 $\mu \mathrm{M}$ Chir99021. We recently named this formulation UPPS medium (Chapter 2). Colony morphology was very similar before and after adaptation (Fig. 1, $\mathrm{C}$ also compare with Fig. 1, A). Both iPSC and ESC colonies present a typical compact structure, regular borders, and a high nucleus/cytoplasm ratio.

DPZ_iRhpb\#1-4 express pluripotency markers OCT4A, LIN28, TRA-1-60, SOX2, TRA-1-81, and SALL4 tested by immunostaining (Fig. 2 A, DPZ_iRhpb\#1-3) (Suppl. Fig. 1, A, DPZ_iRhpb\#4). OCT4A, LIN28, SOX2, and SALL4 were detected in the nucleus, and LIN28 was present in the cytoplasm. TRA-1-60 and TRA-1-81 were present in the membrane. TRA-1-60, TRA-1-81, and SALL4 are pluripotency related markers not encoded by the transposon, indicating successful reprogramming. Isotype controls were performed as negative control (Suppl. Fig. 1, B).

In order to discriminate between the expression of endogenous and exogenous (piggyBac-encoded) pluripotency factors, we performed RT-PCR (Suppl. Fig. 2). Primers were designed to specifically amplify the endogenous transcripts of OCT4A, $S O X 2, N A N O G$, and $c-M Y C$. Primers to evaluate exogenous expression were designed to amplify the fused LIN28-NANOG transcript (Chapter 1). None of the four lines analyzed showed silencing of the reprogramming construct on the transcript level. Nevertheless, all lines showed reactivation of the endogenous pluripotency genes $O C T 4 A$, SOX2, NANOG, and $c-M Y C$. The intensity of the PCR bands representing the endogenous transcripts was similar to the respective bands obtained with Rh_ESC (Suppl. Fig. 2). 
(A)

DPZ_iRhpb\#1 DPZ_iRhpb\#2 DPZ_iRhpb\#3

\section{OCT4A}
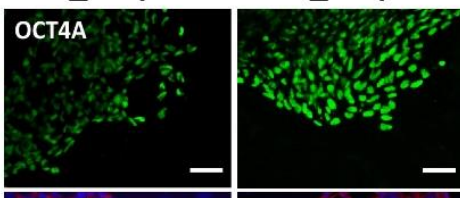

LIN28/DAPI

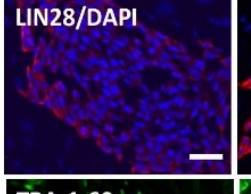

\section{TRA-1-60}
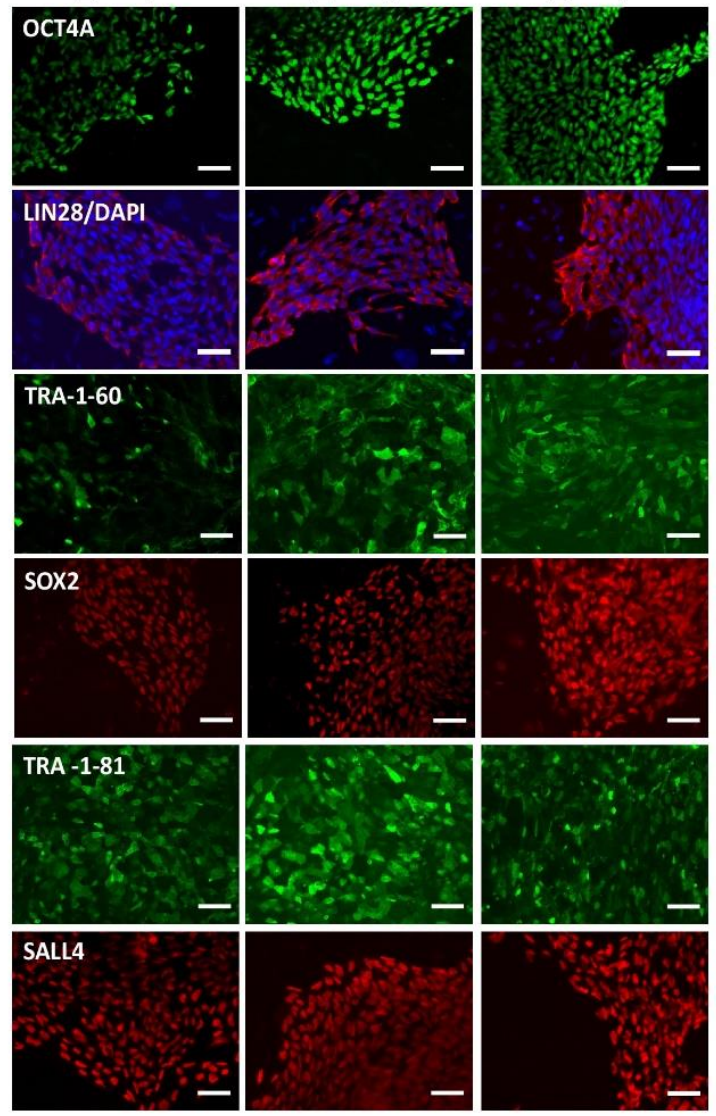

(C)
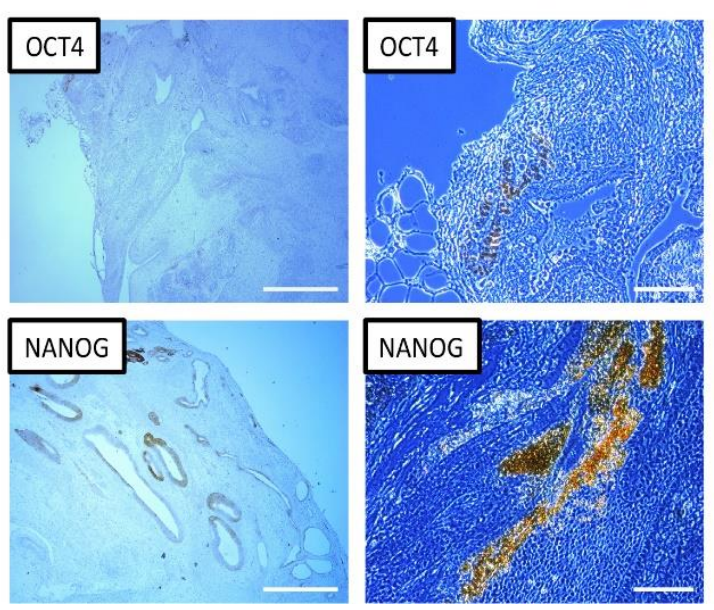

(B)
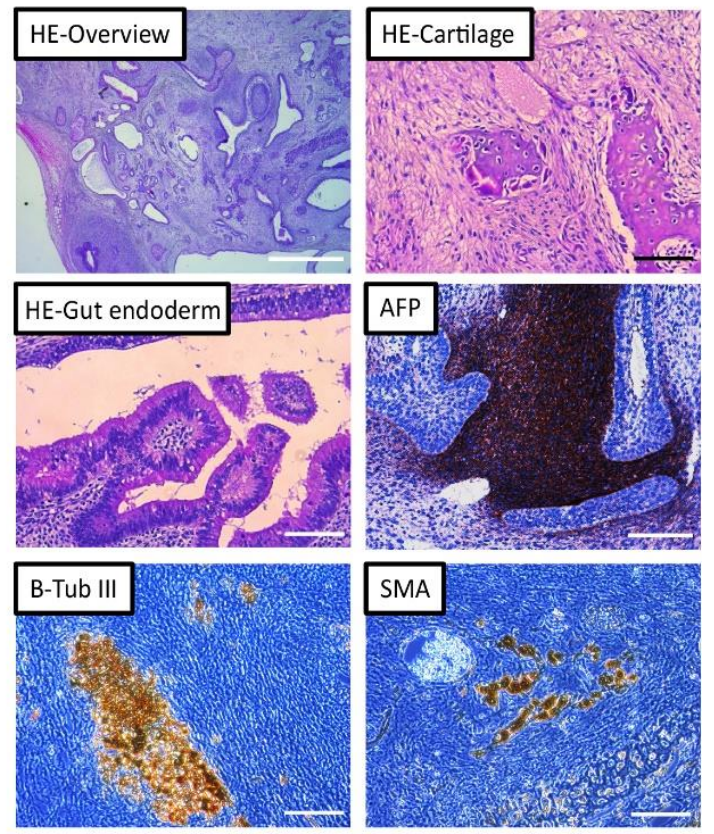
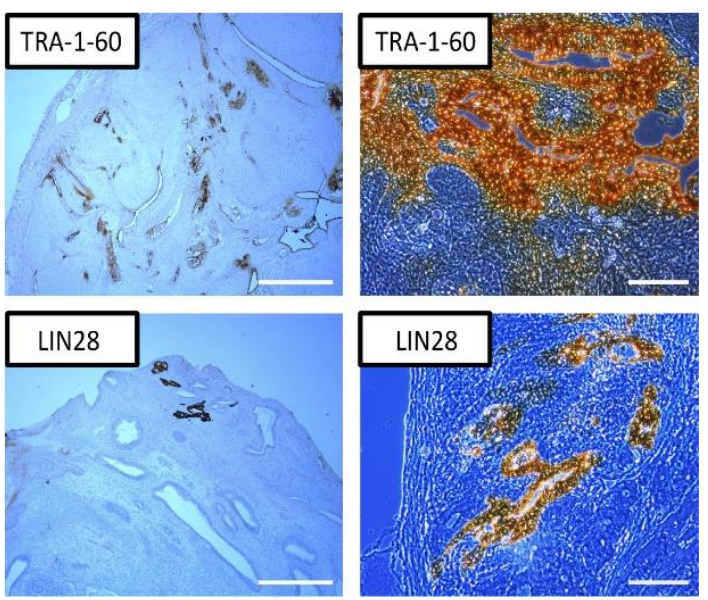

Figure 2: Rhesus iPSC characterization. (A) Characterization by immunofluorescence staining. Staining of Rhesus iPSC colonies of lines DPZ_iRhpb\#1-3. Detection of the pluripotency markers OCT4A, LIN28, TRA-1-60, SOX2, TRA-1-81, and SALL4. TRA-1-81, TRA-1-60, and SALL4 expression is endogenous, as these factors are not contained in the reprogramming construct. The origin

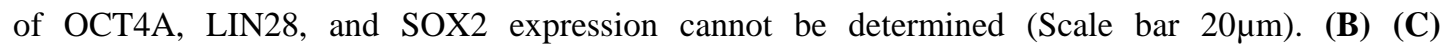
Immunohistochemical analysis of DPZ_iRhpb\#2 teratoma. (B) Teratoma sections were stained for representative markers of the three germ layers: $\beta$ - Tubulin III staining indicates ectodermal 
differentiation. Smooth muscle actin and AFP staining show mesodermal and endodermal differentiation, respectively. Complementarily HE staining was performed to identify specific cytological structures of representative tissues. Presence of cartilage indicates mesoderm, and gut endoderm corroborates endodermal differentiation (Scale bars 100 $\mu \mathrm{m}$ ). (C) Additionally, DPZ_iRhpb\#2 teratoma expressed pluripotency markers OCT4A, NANOG, TRA-1-60, and LIN28. Small isolated clusters were found positive for the pluripotency markers tested (Left picture, scale bar $1 \mathrm{~mm}$, right picture, scale bar $100 \mu \mathrm{m})$.

\subsection{In vivo assessment of pluripotency}

In order to functionally demonstrate the pluripotency of DPZ_iRhpb\#1-4, teratoma formation assay was performed. All four lines formed teratomas (Fig. 2, B, DPZ_iRhpb\#2) (Suppl. Fig. 3 DPZ_iRhpb\#1 and 3) (Suppl. Fig. 4, A, DPZ_iRhpb\#4). All tumors present a high degree of histological heterogeneity, i.e., differentiation. Cartilage, ossification, smooth muscle cells and gut endothelium, indicating mesodermal and endodermal differentiation, respectively, were seen (Fig. 2, B) (Suppl. Fig. 3). Subsequently, stainings for representative markers of each germ layer were performed. Neural tissues positive for $\beta$-Tubulin III, indicating ectodermal differentiation, were identified. In addition, alpha-smooth muscle actin (SMA) and alpha-fetoprotein (AFP) stained clusters that demonstrate mesodermal and endodermal differentiation, respectively (Fig. 2, B) (Suppl. Fig. 3). In summary, the teratoma analysis confirms pluripotency of the generated iPSC.

In order to evaluate if the expression of the pluripotency factors was downregulated during differentiation, teratomas were stained for pluripotency markers. OCT4A, NANOG, TRA-1-60, and LIN28 expression was absent from almost all cells of the teratomas and was limited to a few isolated clusters found in all teratomas (DPZ_iRhpb\#2, Fig. 2, C) (DPZ_iRhpb\#1, DPZ_iRhpb\#3, data not shown). This finding suggests that endogenous (OCT4A, NANOG, TRA-1-60, and LIN28) as well as piggyBac (OCT4A, NANOG, and LIN28) pluripotency factor expression is generally downregulated during differentiation (Fig. 2, C). Isotype controls were performed as negative control (Suppl. Fig, 4, B).

\subsection{PiggyBac silencing during reprogramming and differentiation}

The histological analysis of the teratomas generally suggested silencing of the piggyBac transposon used for reprogramming; most of the cells neither expressed OCT4A nor Nanog. For both proteins, we have established very specific and sensitive IHC detection protocols ${ }^{29}$. In order to clarify if the silencing occurs during reprogramming or differentiation, we performed comparative methylation analysis of the reprogramming construct in iPSCs and teratomas (Fig. 3) (Suppl. Fig. 5). 
The reprogramming construct contains two separate CAG promoters; one driving the reprogramming cassette and one driving the puromycin resistance gene (Fig. 3, A). We aimed at getting an overview of the overall methylation of the construct and to gain insights into possible differential methylation patterns between both promoters (CAG reprogramming vs. CAG puromycin). DNA methylation analysis was performed by bisulfite conversion using primers designed to specifically amplify the promoter driving the expression of the reprogramming 6-factor cassette (CAG reprogramming). Another primer pair was designed to amplify the puromycin resistance promoter (CAG puromycin). Additionally, primers amplifying both promoters non-selectively were used (CAG) (Fig. 3, A). The three amplicons were sequenced with two different oligonucleotides, i.e. S2 (Fig. 3) and S1 (Suppl. Fig. 5). Fibroblasts and rhesus ESCs were included as negative controls.

(A)

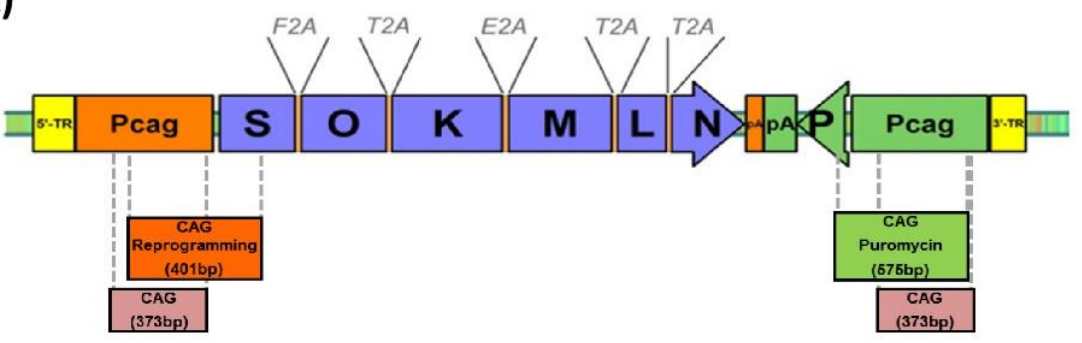

(B)

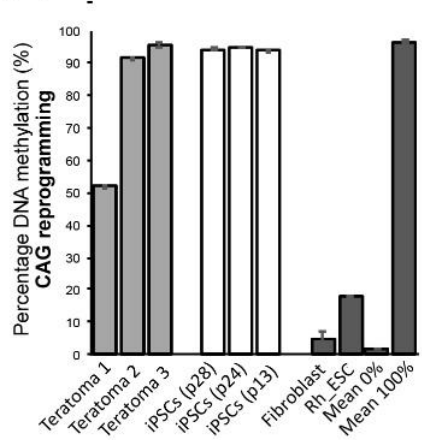

(C)

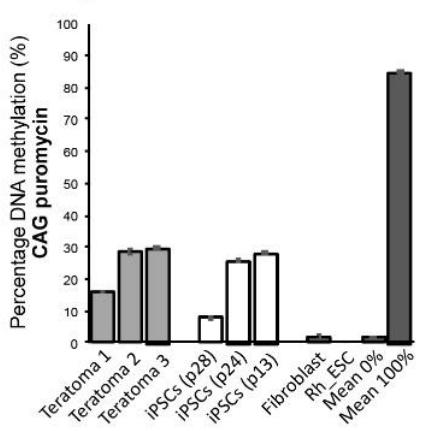

(D)

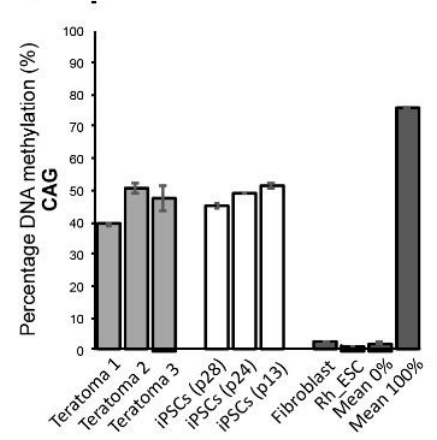

(E)

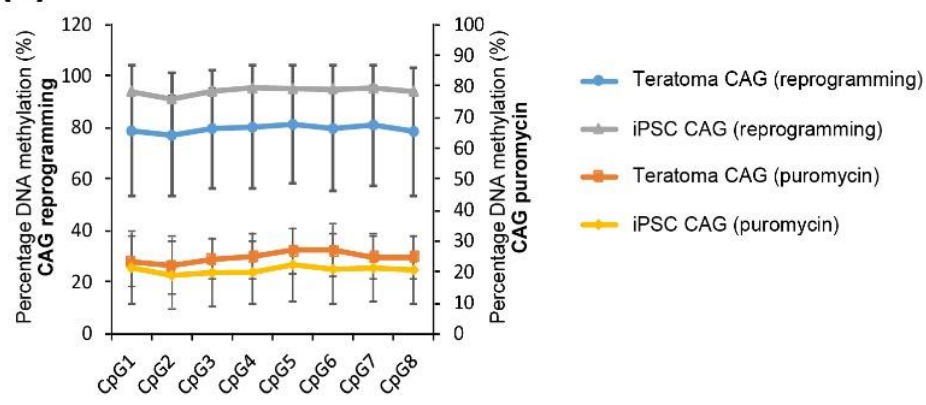

Figure 3: Methylation analysis of the reprogramming construct using sequencing primer S2. (A) PiggyBac reprogramming construct used in this study (13592bp) containing the marmoset (Callithrix jacchus) pluripotency factors, SOX2 (S), OCT4 (O), KLF4 (K), c-MYC (M), LIN28 (L), and NANOG (N) ${ }^{23}$. Stop codons were substituted by $2 \mathrm{~A}$ peptide sequences (F2A, T2A, and E2A). Expression of the reprogramming transcript is driven by a CAG promoter. An independent CAG promoter controls the 
expression of the puromycin resistance gene $(\mathrm{P})$. Below the vector: Schematic representation of the amplicons used for pyrosequencing. Three different primer combinations were used, one to amplify both CAG promoters (CAG) simultaneously, one to amplify specifically the CAG promoter driving reprogramming cassette expression (CAG reprogramming), and one specific for the promoter associated with the puromycin resistance gene (CAG puromycin). (B) (C) (D) Methylation analysis of three teratomas (Teratoma 1-3, DPZ_iRhpb\#1-3), and three iPSC lines (DPZ_iRhpb\#1-3). Two samples were included as negative control: Fibroblasts and Rh_ESCs. Internal technical controls are also shown (Mean 0\% and Mean 100\%) (Mean \pm SD). (B) CAG reprogramming methylation analysis. (C) CAG Puromycin methylation analysis. (D) Non-discriminative CAG promoter methylation analysis. (E) CAG reprogramming vs. CAG puromycin methylation of the $8 \mathrm{CpG}$ sites considered (left y-axis, CAG reprogramming, right $\mathrm{y}$-axis $\mathrm{CAG}$ puromycin). Represented Mean $\pm \mathrm{SD}$ of the teratomas and the iPSC.

Three teratomas and three iPSC lines were included in the analysis. The methylation of the reprogramming cassette promoter (CAG reprogramming, 60-95\% methylation) is higher than the methylation of the puromycin promoter (CAG puromycin, 17-30\% methylation) in all samples (Fig. 3, B, C) (Suppl. Fig. 5, A, B). As expected, the analysis of both promoters (CAG) showed intermediate values. No significant differences were found between teratomas and iPSC for any of the two promoters. These findings suggest that the epigenetic modifications are mainly triggered during reprogramming. GpC island specific evaluation shows homogenous methylation levels at all CpG sites in all amplicons (Fig. 3, D). The two different primers used for sequencing show consistent results (Fig. 3, compare with Suppl. Fig. 5). This data shows that already iPSCs have close to maximum methylation levels of the CAG reprogramming similar to the teratomas. This may explain the down-regulation of the expression of the exogenous pluripotency factors. However, the CAG puromycin promoter is significantly less methylated than the CAG reprogramming promoter demonstrating differential methylation of the two sequence-wise identical CAG promoters present in the piggyBac construct.

\subsection{Transposon removal by re-expression of pBase-dtTomato in iPSCs}

We have shown high methylation of the CAG reprogramming promoter (Fig. 3) (Suppl. Fig.5). However, RT-PCR analysis still detected the transposon-encoded transcript in the iPSC lines (Suppl. Figure 2). In order to generate a transgene-free iPSC line and to demonstrate the stability of the iPSC lines based on endogenous gene expression, we exemplarily removed the reprogramming transposon from DPZ_iRhpb\#4. Cells were re-transfected with the transposase vector pBase-dtTomato (Fig. 4, A) ${ }^{23}$. Two days after transfection, clusters of Tomato-positive cells were identified. Tomato-positive cells were sorted by FACS for selection of the potential transgene-free clones. Cell clones were expanded, and different clones were analyzed for the presence / absence of the transposon by PCR (Fig. 4, D and E). Five different primer pairs were used to detect different fragments of the transposon (Fig. 4, E). One out of 28 clones showed no presence of the reprogramming cassette. 


\section{Chapter 3: A piggyBac-based platform for genome editing and clonal rhesus macaque iPSC line derivation}

The resulting transgene-free subline $\mathrm{DPZ} i \mathrm{Rhpb} \# 4 \Delta \mathrm{pb}$ remained undifferentiated under feeder-free conditions (Fig. 4, B, compare with Fig. 1, C) and full characterization was performed again after transposon removal. iRhpb\#4 $\Delta$ pb showed high alkaline phosphatase activity, and expression of pluripotency markers OCT4A, LIN28, TRA-1-60, SOX2, TRA-1-81, and SALL4 on the protein level (Suppl. Fig. 6, A, B). iRhpb\#4 $\triangle \mathrm{pb}$ also produced teratomas (Suppl. Figure 6, C). To confirm the absence of the transposon on the mRNA level, iRhpb\#4 $\Delta \mathrm{pb}$ was analyzed via RT-PCR. $\mathrm{iRhpb} \# 4 \Delta \mathrm{pb}$ showed no exogenous expression of the LIN28-NANOG fusion transcript, confirming the absence of the transposon-derived mRNA. Additionally, iRhpb\#4 $\Delta \mathrm{pb}$ presents similar levels of endogenous OCT4A, SOX2, NANOG, and $c-M Y C$ expression in comparison with iRhpb\#1-4 (Suppl. Fig. 2). Importantly, no chromosomal rearrangements were detectable by karyotyping in $i R h p b \# 4 \Delta \mathrm{pb}$ after excision of the piggyBac construct (Fig. 4C).

(A)
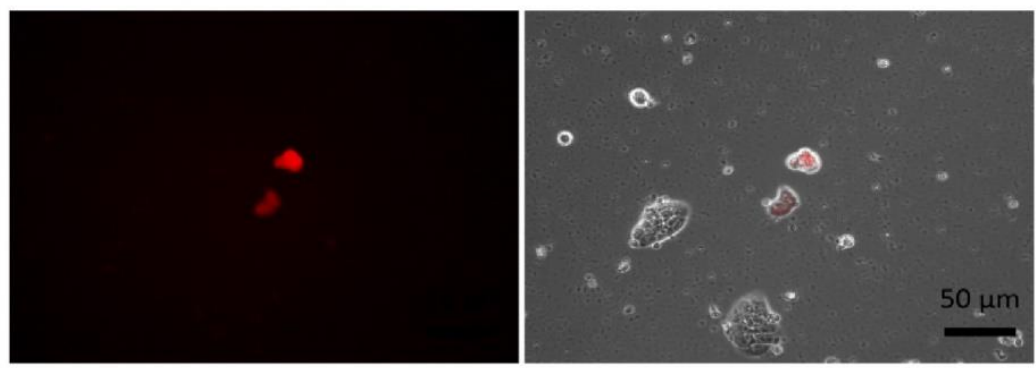

(B)

(C)
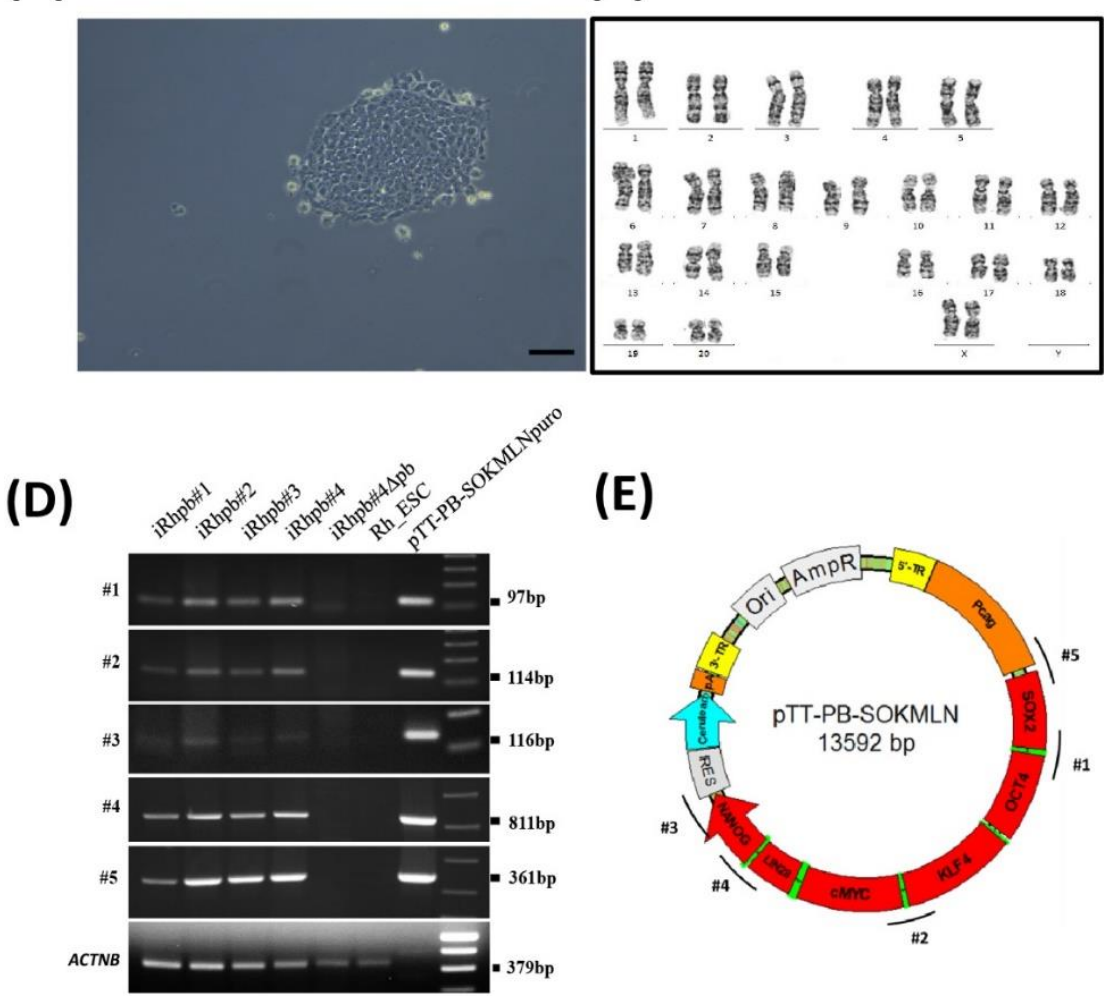

(E)

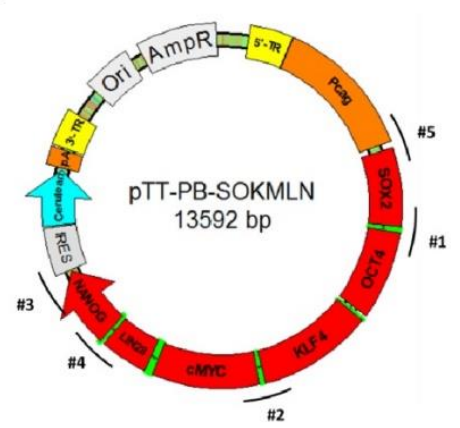

Figure 4: Transposon removal by re-expression of pBase-dtTomato in rhesus iPSC lines. 
(A) iRhpb\#4 two days after transfection with the transposase vector pBase-dtTomato. Left, red fluorescence channel; right, merged brightfield / fluorescence. Tomato positive clones were sorted and isolated in order to check for the removal of the transposon (Scale bar 100 $\mu \mathrm{m}$ ). (B) Brightfield image

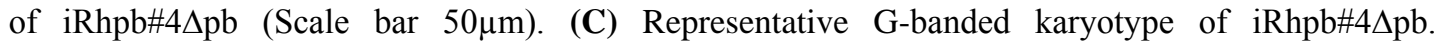
Karyotyping yielded a normal female rhesus macaque chromosome set without any numerical or structural abnormality. (D) (E) Absence of the piggyBac construct from clone iRhpb\#4 $\Delta \mathrm{pb}$. PCR amplicons for the detection of the reprogramming vector are schematically represented (\#1-5). Betaactin was used as control for the presence of gDNA $(A C T N B)$. Positive controls used for the analysis

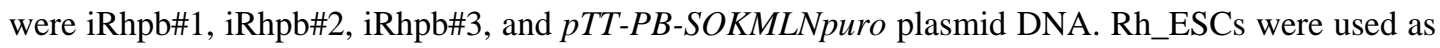
negative control.

In summary, marker expression and teratoma formation show that $\mathrm{iRhpb} \# 4 \Delta \mathrm{pb}$ remains pluripotent under feeder-free conditions and has a normal karyotype (Fig. 4) after removal of the transposon.

\subsection{Derivation of clonal rhesus macaque pluripotent stem cell lines}

To generate clonal iPSC lines with defined mutations, single-cell isolation and propagation protocols need to be established. Two cell lines, Rh_ESC and DPZ_iRhpb\#4 were selected to develop a work-flow for clonal PSC line generation (Fig 1, C).

The first step towards single-cell cloning is reliable and complete dissociation of cell clusters to a single cell suspension. In order to find the most suitable reagent, we tested three reagents commonly used in human PSC protocols: versene, accutase, and TrypLE Select (Fig, 5, A). After dissociation with the different reagents, we analyzed proliferation and differentiation of the cells. Five days after splitting, differentiation of Rh_ESC and DPZ_iRhpb\#4 was assessed by alkaline phosphatase staining and proliferation by cell counting. The starting number was 200.000 cells. No differentiation was found for any of the three tested reagents (Fig, 5, B). Proliferation analysis of DPZ_iRhpb\#4 reveals no significant differences between the dissociation reagents. In contrast, Rh_ESCs dissociated with accutase or versene show higher proliferation recovery in comparison with TrypLE Select (Fig, 5, C). The data collected suggest that accutase or versene are more suitable reagents for single-cell dissociation compared to TrypLE for Rh_ESCs.

Subsequently, we transfected Rh_ESC and DPZ_iRhpb\#4 with the vector $p T T-P B$ pCAG-eCas9-GFP-U6-gRNA-Neo together with pBase-dtTomato encoding the transposase (Fig. 6, A). Three passages after transfection single cells were sorted into a 96-well plate by FACS (one and three cells / well, respectively). This approach allowed us to expand single clones and to obtain a representative number for analysis. We tested three different conditions to improve clonal expansion. Firstly, culture with and without pro-survival factor (PSF), secondly, with and without Activin A and, thirdly, under normoxia and hypoxia (5\% O2). Only PSF significantly increased cell 


\section{macaque iPSC line derivation}

survival and / or proliferation (Fig, 5 D), while neither Activin supplementation for two days after sorting (Fig, 5 E) nor hypoxia (Fig, 5 F) significantly improved clonal expansion. In summary, the use of accutase in combination with medium supplemented with PSF for clonal expansion provides optimized conditions for the establishment of clonal rhesus PSC lines.

(A)

\begin{tabular}{|c|c|c|}
\hline Dissociation reagent & Enzymatic $(\mathrm{Y} / \mathrm{N})$ & Single cell dissociation \\
\hline Versene & $\mathrm{N}$ & $37^{\circ} \mathrm{C}, 5 \mathrm{~min}$ \\
Accutase & $\mathrm{Y}$ & $37{ }^{\circ} \mathrm{C}, 5 \mathrm{~min}$ \\
0,75X TrypLE-Select & $\mathrm{Y}$ & $37^{\circ} \mathrm{C}, 3 \mathrm{~min}$ \\
\hline
\end{tabular}

(B)
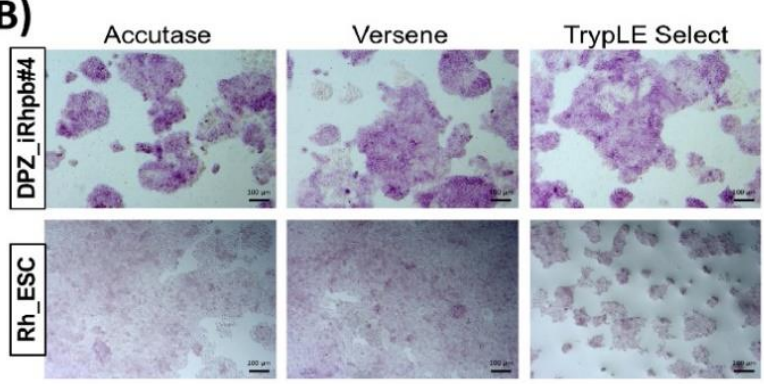

\section{(C)}

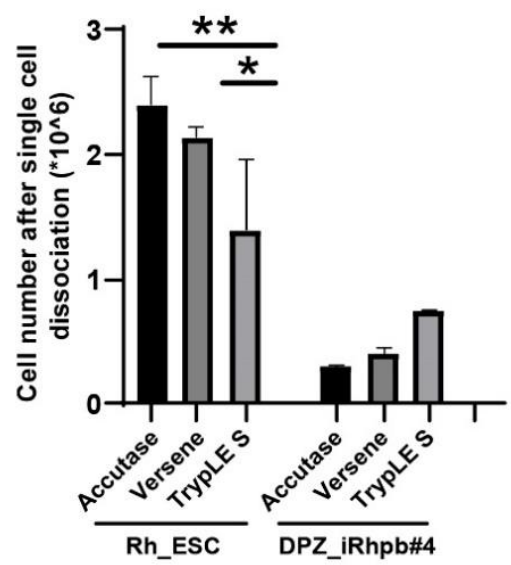

(D)

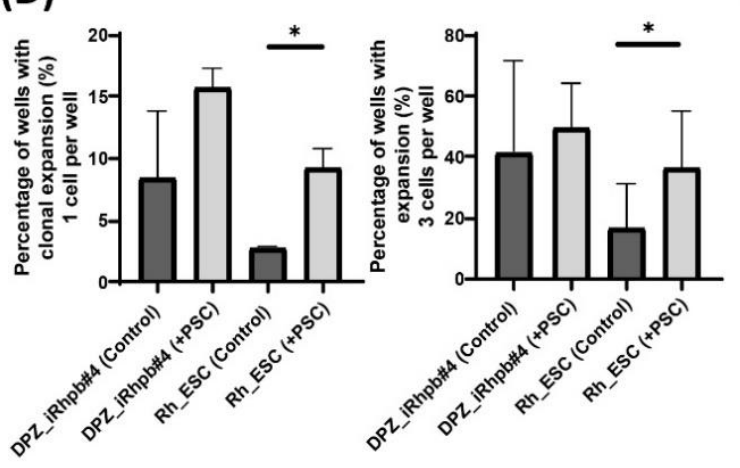

(E)

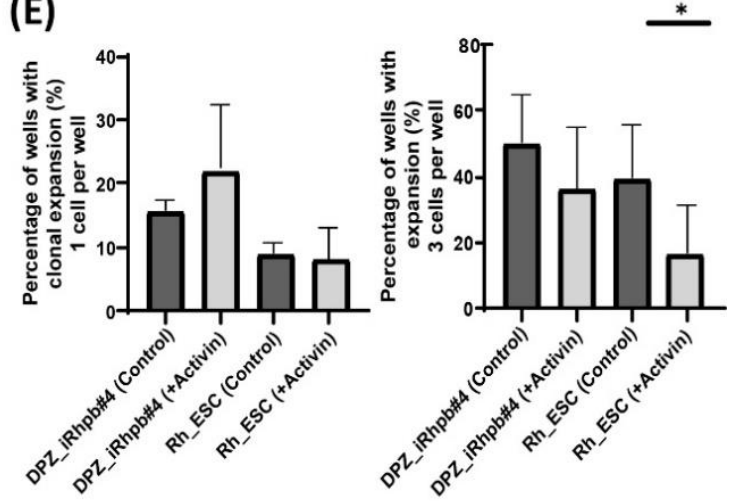

(F)
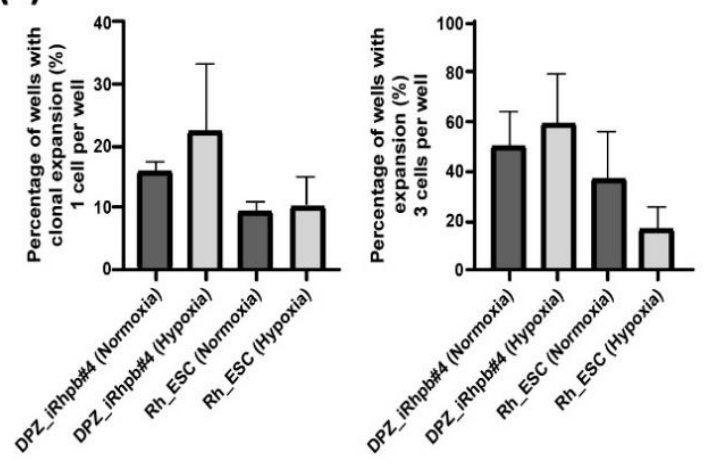

Figure 5: $(\mathbf{A})(\mathbf{B})(\mathbf{C})$ Clonal cell line derivation from rhesus macaque pluripotent stem cells. (A) Reagents and conditions tested for dissociation. (B) Alkaline phosphatase staining after single-cell dissociation and re-seeding of rhesus macaque PSCs, DPZ_iRhpb\#4 and Rh_ESC (Scale bar $100 \mu \mathrm{m})$. (C) Cell numbers three days after single-cell dissociation of $2 * 10^{5}$ initial cells $(n=4)($ Mean \pm SD). (D)(E)(F) Percentage of cells (iRhpb\#4 and Rh_ESC) achieving clonal expansion after dissociation and sorting in 96 well plates. Left graph, clonal expansion after sorting one cell per well. Right graph, colony expansion after sorting three cells per well (Mean \pm SD). (D) Cells sorted in standard UPPS medium (Control) and in UPPS medium 


\section{Chapter 3: A piggyBac-based platform for genome editing and clonal rhesus macaque iPSC line derivation}

supplemented with $5 \mu \mathrm{M}$ pro-survival factor (+PSF). (E) Clonal expansion of cells sorted in UPPS $+5 \mu \mathrm{M}$ PSF in normoxia and hypoxia $\left(5 \% \mathrm{O}_{2}\right)$. (F) Percentage of wells with clonal expansion of cells sorted in UPPS + PSF (Control) vs. cells sorted in UPPS +PSF +Activin (50

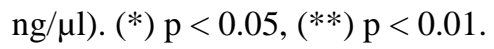

\subsection{Genome editing of rhesus macaque stem cells using the piggyBac transposon Cas9/GFP vector}

In order to validate the cloning protocol, we designed and tested CRISPR/Cas9 guides to target clinically relevant mutations in the sarcomeric gene Titin (TTN). Two locations were targeted, one at the $\mathrm{N}$-terminus and another one at the $\mathrm{C}$-terminal part of the protein (rhesus exons homologous to human TTN exons 38 and 280, respectively; ENST00000589042.5). Guides were inserted in the pTT-PB-pCAG-eCas9-GFP-U6$g R N A-N e o$ vector and transfected together with the transposase. The single-cell cloning protocol developed above was followed, and mono-clonal lines were expanded. Four clones with truncating mutations at the N- terminus of TTN, two for Rh_ESC and two for DPZ_iRhpb\#4, were obtained. The efficiency of the process was systematically analyzed for the guide targeting the C-terminus, and independent biological replicas for each cell line were performed (transfection, sorting, and expansion). For the $\mathrm{C}$-terminus mutation, 37 mutated clones were obtained from 42 clones analyzed in total (DPZ_iRhpb\#4: 27 mutated / 28 analyzed; Rh_ESC: 10 mutated / 14 analyzed). The overall efficiency of the process for this gRNA was $85 \%$ (mutated clones / analyzed clones) (Fig. 6, C and D). In conclusion, we established tools and a protocol for the highly efficient generation of genetically modified clonal rhesus macaque ESC and iPSC lines carrying clinically relevant mutations. Preliminary characterization of the modified sequences was done exemplarily by sequencing of two clones, one mutated at the $\mathrm{N}$-terminus and the other one mutated at the C-terminus (Fig. 6, D). 
(A)
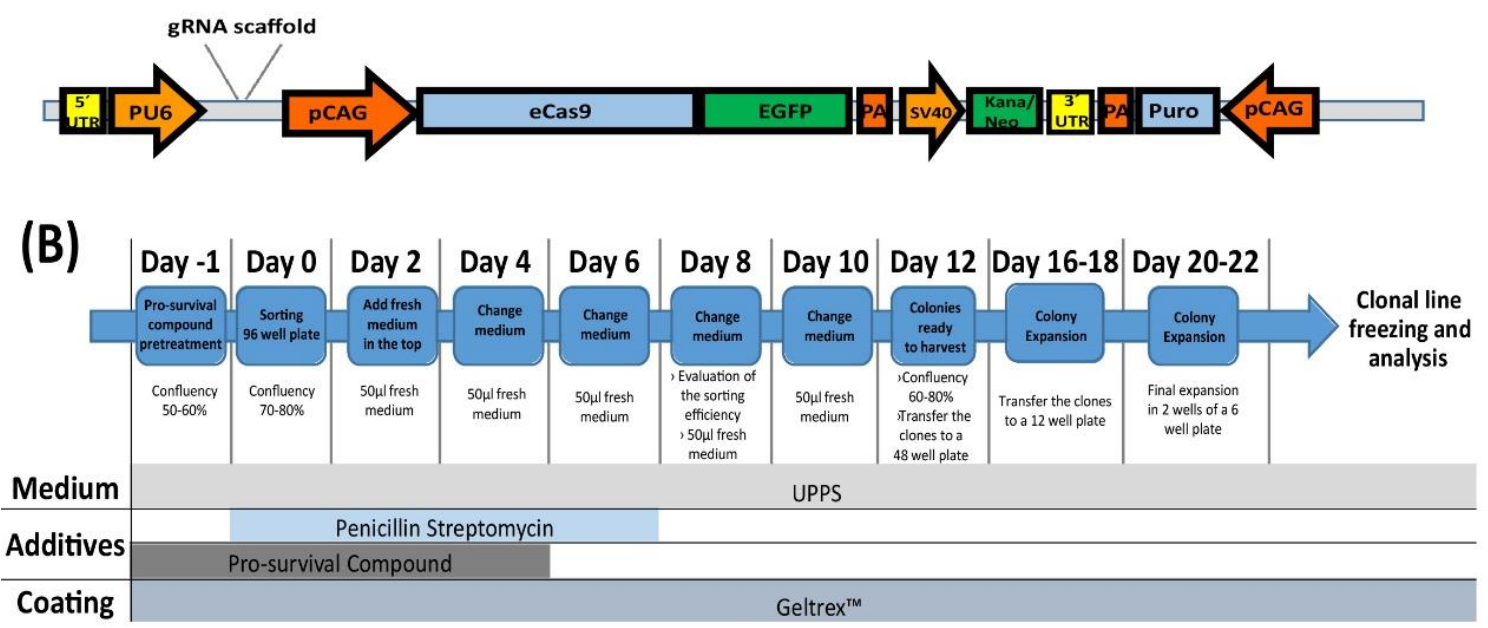

(C)

\begin{tabular}{ccccc} 
& $\begin{array}{r}\text { \# of clones } \\
\text { analyzed }\end{array}$ & $\begin{array}{c}\text { \# of clones } \\
\text { mutated clones }\end{array}$ & $\begin{array}{c}\text { Editing } \\
\text { efficiency (\%) }\end{array}$ \\
\hline Rh_ESC & 1 & 6 & 3 & 50 \\
\hline Rh_ESC & 2 & 8 & 7 & 87.5 \\
\hline iRhpb\#4 & 1 & 9 & 8 & 88.9 \\
\hline $\mathrm{iRhpb \# 4}$ & 2 & 9 & 9 & 100 \\
\hline $\mathrm{iRhpb \# 4}$ & 3 & 10 & 10 & 100 \\
\hline \multicolumn{5}{c}{ Average editing efficiency $=85.3 \%$} \\
\hline
\end{tabular}

(D)

WT
Allele A
Allele B

$W T$
Allele A
Allele B

TTN N-terminal mutation

CACAGTTACTGCTTCCAATCGCCATGGCTCA CACAGTTACTGGCTCCAATCGCCATGGCTCA + +-1bp CACAGTTACTGGCTTCCAATCGCCATGGCTCA $+1 \mathrm{bp}$

ITN C-terminal mutation

TIITGAGGTGACAGTG -.AACTATGAAGGCAT TITIGAGGTGACAGTGCTAACTATGAAGGCAT +2bp TITTGAGGTGACAG-G----AACTATGAAGGCAT

Figure 6: Genome editing of rhesus macaque pluripotent stem cells using the piggyBac system. (A) Schematic representation of the piggyBac vector used for CRISPR/Cas9 expression, $p T T-P B-p C A G$ eCas9-GFP-U6-gRNA-Neo (13213bp). Three independent expression units are contained in the piggyBac construct. 1. U6 promoter regulating the expression of tRNA-gRNA, 2. CAG promoter-driven expression of eCas9-eGFP fusion transcript. Both coding sequences are separated by a 2TA sequence, and 3. SV40 promoter driving the expression of the Kanamycin/Neomycin resistance gene. (B) Singlecell cloning workflow. Detailed in the graph are the medium used, coating of the cell culture dish, and additives to the medium, together with a simplified overview of the cloning process. (C) Genome editing efficiency of the TTN C-terminus. Five independent experiments were performed: 2 with Rh_ESC and 3 with iRhpb\#4. All transfections led to mutated clones with an efficiency ranging from 50\% to $100 \%$. Overall, mutation efficiency was $85,3 \%$. (D) Mutation analysis of two clones of iRhpb\#4, one with a mutation in the $\mathrm{N}$-terminus, and the other in the $\mathrm{C}$-terminus. The top line shows the wildtype sequence, the middle and the bottom sequences the two mutated alleles in the two selected clones.

\subsection{Isogenic control generation}

The next step after the generation of the mutated clones will be to assess the phenotypic alteration in functional cells generated from the pluripotent stem cells lines, e.g., iPSCderived cardiomyocytes. Downstream analysis of the mutated cells requires the generation of proper isogenic controls, to exclude possible effects of the cloning process. Therefore, isogenic controls were generated for both lines. Transfection of the cells with the piggyBac Cas9 vector without guides (pTT-PB-pCAG-eCas9-GFP-U6$g R N A-(e o$ ), but also with transposase was performed. Isolation of appropriate isogenic clonal control populations expressing the Cas9-GFP construct was achieved for Rh-ESC and DPZ_iRhpb\#4 (Suppl. Fig. 7). 


\section{Materials and Methods}

\subsection{Animals and animal housing; ethics statement}

The German Primate Center (DPZ) is authorized by the local and regional veterinary governmental authorities (Reference number: 122910.3311900, PK Landkreis Göttingen). Rhesus macaque skin samples were made available during necropsy, from animals kept in the context of an unrelated project. DPZ_iRhpb\#1-3 iPSC were derived from an adult male macaque (16 years), and DPZ_iRhpb\#4 iPSC from a female (8 years).

\subsection{Isolation of rhesus macaque primary fibroblasts}

Isolation of rhesus macaque fibroblasts from skin and gingiva biopsies was performed according to ${ }^{30}$. In brief, a tissue biopsy of approximately $1 \mathrm{x} 1 \mathrm{~cm}$ was washed with PBS (1\% (v/v) Penicillin/Streptomycin (Gibco), 0,25 $\mu \mathrm{g} / \mathrm{mL}$ Amphotericin B (Sigma)) and subcutaneous adipose tissue was removed with a scalpel. Clean dermal tissue was chopped with scissors into small pieces and incubated in Collagenase type IV solution $\left(10 \mathrm{mg} / \mathrm{mL}\right.$ in DMEM) (Gibco) for three hours at $37^{\circ} \mathrm{C}$ rotating $(80 \mathrm{rpm})$. After digestion, cells were pelleted by centrifugation $(5 \mathrm{~min}, 300 \mathrm{~g}, \mathrm{RT})$. Fibroblasts were seeded in $10 \mathrm{~cm}$ diameter gelatine-coated culture dishes $(0,1 \%$ gelatine; Sigma) and cultured in Rh15 medium [DMEM (Gibco), 15\% (v/v) Fetal Bovine Serum (Gibco), $1 \%(\mathrm{v} / \mathrm{v})$ Penicillin/Streptomycin (Gibco), 0,1\% (v/v) Amphotericin B (Sigma), $1 \%$ (v/v) MEM Non-Essential Amino Acids Solution (Gibco), 2 mM GlutaMAX (Gibco)] at $37^{\circ} \mathrm{C}$ and $5 \% \mathrm{CO} 2$. Accutase dissociation reagent (Gibco) was used for passaging.

\subsection{Mouse embryonic fibroblasts (MEFs)}

Gamma-irradiated MEFs were used as feeder cells. The generation was described previously ${ }^{23}$.

\subsection{Nucleofection and reprogramming procedure}

Primary fibroblasts were reprogrammed using the 6-factors in one vector piggyBac construct, coding for the marmoset monkey reprogramming factors SOX2, OCT4, KLF4, c-MYC, NANOG, and LIN28. $1 * 10^{6}$ fibroblasts were transfected with a 4Dnucleofector device (program CA-137; Lonza), using P2 nucleofection solution. The 
reprogramming transposon vector was co-transfected with pBase-dtTomato transposase vector $(9 \mu \mathrm{g}$, and $6 \mu \mathrm{g}$ pDNA, respectively). The efficiency of the nucleofection was estimated two days after transfection by the expression of the Tomato reporter from the transposase vector.

Two days after transfection antibiotic selection was started adding 1,5 $\mu$ g puromycin (Sigma) to the fibroblast culture medium. Five days after selection the fibroblasts were plated $\left(0,2 * 10^{5}\right.$ cells per plate) on gelatine coated $10 \mathrm{~cm}$ plates with MEFs and cultured in embryonic stem cell medium (ESM) [KO-DMEM (Gibco), 20\% (v/v) KnockOut Serum Replacement (Gibco), 1\% (v/v) Pen/Strep (Gibco), 0,25 $\mu \mathrm{g} / \mathrm{mL}$ Amphotericin B (Sigma), 1\% (v/v) MEM (Gibco), 2 mM GlutaMAX (Gibco), $50 \mu \mathrm{M} 2$ mercaptoethanol (Gibco), $10 \mathrm{ng} / \mathrm{ml}$ FGF (ThermoFisher)]. ESM was supplemented with $2 \mathrm{mM}$ valproic acid (Calbiochem) for the first 6 days of culture. 20 days after transfection first colonies were identified and new colonies appeared until day 60 . Colonies were manually picked and transferred to new plates with MEFs. Around passage 5, mass passage was possible using Collagenase type IV $(1 \mathrm{mg} / \mathrm{mL})$. Rhesus macaque iPSC long-term storage was done using Rh_freezing medium [ESM, $20 \%$ DMSO (Sigma), $10 \%$ FBS (Gibco)] at $-150^{\circ} \mathrm{C}$.

\subsection{Adaptation to feeder-free culture conditions}

Rhesus iPSC (iRhpb\#4) in passage 10 were manually picked and transferred into $6 \mathrm{~cm}$ plates coated with Geltrex $(0.16 \mathrm{mg} / \mathrm{ml})$ (Thermo Fisher Scientific). Cells were cultured according to our previously published protocol (Chapter 2). NHP pluripotent stem cells medium UPPS [StemMACS ${ }^{\mathrm{TM}}$ iPS-Brew XF, human - Stem cell media (Miltenyi Biotec) supplemented with IWRI (1 $\mu \mathrm{M})$ (Sigma), and Chir99021 (0.5 $\mu \mathrm{M})$ (Merck/Millipore)]. The first day after picking, the medium was supplemented with ROCK inhibitor, PSF (Pro-survival Compound, $5 \mu \mathrm{M}$, Calbiochem). After 3 to 5 passages, the culture stabilized showing no morphological differentiation. After 5 passages cells were split using versene-EDTA solution (Thermo Fisher) (split ratios used were $1 / 10$ to $1 / 15$ ). To freeze rhesus iPS, the freezing medium was used (Essential

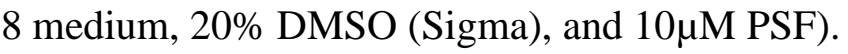

\subsection{Alkaline phosphatase}

Alkaline phosphatase activity was demonstrated using Leukocyte Alkaline Phosphatase Kit (Sigma), following manufacturer's recommendations.

\subsection{Immunofluorescence}

Rhesus iPSCs were cultured in wells equipped with coverslips. When the colonies reached 30 to $50 \%$ confluence, they were fixed with $4 \%$ PFA (v/v) for $20 \mathrm{~min}(\mathrm{RT})$. 
Fixation solution was removed and cells washed three more times with PBS. 1\% BSA in PBS was used for blocking. For intracellular epitopes $1 \%$ BSA, TritonX-100 (0,1\%, Sigma) was used. Primary antibodies were diluted in PBS/ $1 \%$ BSA and incubated for 1 hour at $37^{\circ} \mathrm{C}$. Subsequently, cells were washed with PBS and incubated with Alexa 488-coupled secondary antibodies (Life Technologies) diluted in PBS/1\% (w/v) BSA. Finally, coverslips were incubated with PBS/DAPI solution $(0,5 \mu \mathrm{g} / \mathrm{ml})$, for $10 \mathrm{~min}$. After staining the coverslips were removed from the cell culture wells, and mounted using Citifluor mountant medium (CITIFLUOR ltd.). Immunofluorescence images were taken with a Zeiss Observer Z1 (Zeiss). Primary antibodies used were OCT4 (Cell Signalling OCT-4A C52G3, 1:1600), SOX2 (Cell Signalling C70B1, 1:200), NANOG (Cell Signalling D73G4, 1:400), TRA-1-60 (eBioscience 14-8863, 1:100), TRA-1-81 (eBioscience 14-8883, 1:100), LIN28 (Cell Signalling A177, 1:100), and SALL4 (Abcam ab57577, 1:200). Secondary antibodies Alexa-488 conjugated Ab Donkey anti-Mouse IgG (H+L) (Life technologies A21202, 1:1000), Donkey antiRabbit IgG (H+L) (Invitrogen A-21206, 1:1000) and Goat anti-mouse IgM (H+L) (Invitrogen A-21042, 1:1000).

\subsection{Reverse transcription PCR}

RNA was extracted from cell pellets using RNAeasy Mini Kit (Qiagen). Contaminating genomic DNA was eliminated by treating the samples with RNase-free DNase (Qiagen). cDNA was synthesized from $1 \mu \mathrm{g}$ RNA using Oligo(dT) primers and the Omniscript RT Kit (Qiagen), according to manufacturer instructions. Primer pairs (Sigma) were designed and used for detection of the different pluripotency factors, and to discriminate between the endogenous (OCT4A, SOX2, NANOG, and $c-M Y C$ ) and the exogenous (LIN28-NANOG fusion) transcripts (Table 2). As positive and negative controls rhesus embryonic stem cells (Rh_ESC) and MEFs, respectively, were used. Endogenous beta-actin (ACTNB) expression was used as a house-keeping gene. Taq DNA Polymerase with Standard Taq Buffer (New England BioLabs) was used for all RT-PCRs performed. Primers used are detailed in Table 2.

\subsection{Teratoma formation and histological analysis}

For teratoma formation assay, $8 * 10^{5}$ cells from each iPSC line and $2 * 10^{5}$ MEFs were co-injected subcutaneously into male immunodeficient RAG2-/- $\gamma c-/-m i c e . ~ B e f o r e$ injection cells were resuspended in PBS supplemented with Geltrex (ThermoFisher, $0.1 \mathrm{mg} / \mathrm{mL}$ ), with a final injected volume of $120 \mu \mathrm{l}$. Teratomas were obtained and fixed as previously described ${ }^{31}$. Histological tissue sections were stained for the detection of representative markers of each germ layer. Primary antibodies used were $\beta$-Tubulin III (Sigma, T8660, 1:600), smooth muscle actin (SMA; Sigma, A2547, 1:1000) and SOX9 (Millipore, AB5535, 1:1000) for detection of ectoderm, mesoderm, and endodermal epithelium respectively. Additionally, OCT4A (Cell Signalling OCT-4A C52G3, 1:1000), NANOG (Cell Signalling D73G4, 1:400), TRA-1-60 (eBioscience 
14-8863, 1:50) and LIN28 (Cell Signalling A177, 1:100) primary antibodies were used for the detection of undifferentiated pluripotent cells in the teratomas.

\subsection{Promoter methylation assay}

Promoter methylation analysis was performed in three different iPSC (DPZ_iRhpb\#13 ) at different passages and in three teratomas (DPZ_iRhpb\#1-3). As negative controls, Rh_ESC and rhesus macaque fibroblasts were included in the analysis. DNA methylation analysis of the CAG promoter was performed by bisulfite pyrosequencing. $500 \mathrm{ng}$ of the isolated genomic DNA was used for the DNA bisulfite conversion reaction with the EZ DNA Methylation-Direct ${ }^{\mathrm{TM}}$ Kit (Zymo), according to manufacturer's instructions.

For PCR, Fast Start Taq polymerase (Roche) was used to amplify the bisulfiteconverted DNA. PCR conditions and primers were designed with the Pyrosequencing Assay Design Software (Qiagen). The piggyBac 6-factor reprogramming construct contains two CAG promoters, one driving the expression of the reprogramming cassette, and the other one the puromycin resistance gene. In order to analyze both promoters separately, one assay for each CAG region was designed. Both assays contained the same forward primer but a different reverse primer located in the unique sequence of the reprogramming cassette (CAG Reprog) and the puromycin resistance gene (CAG Puro). In addition, a third assay was designed to evaluate both CAG promoters (CAG). For reducing the amplification bias, all three measurements were arranged in a nested/semi-nested approach consisting of two following PCRs for each assay. The first PCR round generates three different outer amplicons related to the three different assays (CAG Reprog, CAG Puro and CAG) (Table2) (Fig. 3). In the second PCR round, one primer combination of two primers located within the CAG promoter sequence was applied to all three different outer amplicons (CAG Reprog, CAG Puro and CAG) and generate the same inner amplicon for all three assays. This inner amplicon was sequenced with two sequencing primers, which together covered $15 \mathrm{CpGs}$ in total (S1: $7 \mathrm{CpGs}$; 2 : $8 \mathrm{CpGs)}$ ). As cycler conditions of the outer-PCR reactions, the standard PCR procedure according to manufacturer's instruction was applied with a cycle number of 30 (annealing CAG Reprog: $60{ }^{\circ} \mathrm{C}$; CAG Puro: $64{ }^{\circ} \mathrm{C}$; CAG: $52{ }^{\circ} \mathrm{C}$ ) (Table 2). The inner-PCR contained for all three assays the same combination of two primers. The cycler conditions of the inner-PCR corresponded to the standard PCR procedure according to manufacturer's instruction (annealing $53{ }^{\circ} \mathrm{C}$ and 35 cycles). Finally, the products of the inner-PCR were sequenced in triplicates by Pyrosequencing following the manufacturer's instruction. $10 \mu \mathrm{L}$ of the generated inner-PCR products were used for the immobilization to Streptavidin Sepharose HP beads (GE Healthcare Life Sciences). The Pyrosequencing was performed on a Pyromark Q96MD system (Qiagen) and analyzed with the Pyromark Q-CpG software. 


\subsection{PiggyBac transposon removal/rhesus iPSC transfection}

iRhpb\#4 in feeder-free conditions was nucleofected using 4D-nucleofector device (Lonza). $1 * 10^{6}$ cells were transfected with pBase-dtTomato transposase vector $(6 \mu \mathrm{g})$, using P3 nucleofection solution and program CA-137 (Lonza). UPPS medium was supplemented for 2 days with PSF $(5 \mu \mathrm{M})$ after transfection. After 3 days Tomato positive clones were sorted using SH800S Cell Sorter (Sony Biotechnology), sorting 10 cells per well in a 48 well plate. Potential transgene-free colonies were picked and expanded for 2-5 passages. Colonies were then pelleted and gDNA was extracted. Detection PCR was performed using primers specifically designed to detect different regions of the transposon (Table 2). PCR was performed using Taq DNA polymerase with Standard Taq Buffer (New England BioLabs) according to manufacturer instructions. Transgene-free clones were checked for the absence of the reprogramming construct every 10 passages.

\subsection{Karyotyping}

Metaphase arrest, fixation, $\mathrm{G}$ banding, and analysis protocols were described in detail in ${ }^{30}$.

\subsection{PiggyBac CRISPR/Cas vector generation}

The $p T T-P B-p C A G-e C a s 9-G F P-U 6-g R N A-N e o$ vector was derived by modification of plasmid $p C A G-e C A S 9-G F P-U 6-g R N A$, which was a gift from Jizhong Zou (Addgene plasmid \# 79145; http://n2t.net/addgene:79145; RRID: Addgene_79145). In order to convert this plasmid into a piggyBac transposon, the 5'- and 3'-PB inverted repeats were amplified from $p T T-P B-S O K M L N$-Puro by PCR and inserted respectively into the PacI and NotI/SbfI restriction sites using standard molecular cloning techniques. Additionally, a neomycin-resistance cassette was inserted into the BsrGI restriction site in order to enable antibiotic selection.

\subsection{Dissociation reagent testing}

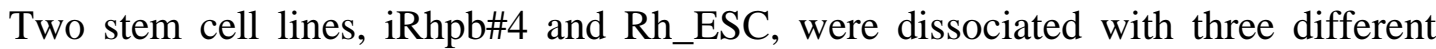
reagents. Dissociation conditions are listed in detail in Fig. 5A. After dissociation, cells were collected and centrifuged (180g, 5min, RT). After centrifugation 200,000 cells were transferred into a well of a 6-well plate and cultured for 5 days. Alkaline phosphatase staining and cell counting were subsequently performed. Statistical analysis of the cell numbers was performed using GraphPad PRISM and one way ANOVA test $[(*) \mathrm{p}<0.05,(* *) \mathrm{p}<0.01$.$] .$ 


\subsection{Clonal expansion analysis}

iRhpb\#4 and Rh_ESC were used for the testing of the clonal expansion potential after single-cell dissociation and FAC-sorting. Cells transfected with $p T T-P B-p C A G-$ eCas9-GFP-U6-gRNA-Neo were cultured for three passages and digested with accutase $\left(37^{\circ} \mathrm{C}, 5 \mathrm{~min}\right)$. The SH800S Cell Sorter (Sony Biotechnology) was used to sort Cas9-GFP positive cells. To evaluate the effect of PSF during clonal expansion, a control group was sorted with no PSF and compared to a test group with PSF. For the evaluation of the effect of Activin A ( $50 \mu \mathrm{g} / \mu \mathrm{l}$; Miltenyi), or hypoxia (5\% O2), the different experimental groups were compared to each other. Since PSF turned out in the first experiment to support clonal cell line derivation, it was the standard based on which the remaining variables (Activin and hypoxia) were tested. All experiments were performed in triplicates. For the significance analysis, paired T-test was performed using GraphPad PRISM software.

\subsection{Induction of truncation mutations in rhesus macaque stem cells}

Two different guide RNAs for the CRISPR/Cas9 system were cloned (vectors digestion with $B b s \mathrm{I}$, plus oligo alignment and ligation) and validated in the $p T T-P B$ pCAG-eCas9-GFP-U6-gRNA-Neo vector (Table 2). Transfection of the iPSC was described above (piggyBac transposon removal/rhesus iPSC transfection). Cells were FACS-sorted in order to obtain clonal sublines, expanded, and pelleted for gDNA extraction and analysis (Macherey-Nagel ${ }^{\mathrm{TM}}$ NucleoSpin $^{\mathrm{TM}}$ Gel and PCR Clean-up Kit). Primers for the mutation site amplification and PCR conditions are described in Table 2. PCR products of the different clones were purified and sent for sequencing (LGC Genomics). Sequence analysis was performed using Serial Cloner software. Mutations in the two different alleles of the target gene of each clone were predicted using Poly Peak Parser ${ }^{32}$.

\section{Discussion}

NHP as our closest relatives are excellent animal models to study human diseases. The phylogenetic relationship is reflected in similar physiology, genetics, life span, and relatively equal size 56891011 . In order to model human pathologies with genetic origin in NHP, it is necessary to edit the embryonic genome. Therefore genome editing tools for these applications need to be validated in vitro before in vivo translation to guarantee the efficiency, accuracy and hence safety of the process. NHP-PSC share many molecular signatures of the cells present in the early embryo. Therefore, testing of novel editing tools in PSCs will help to assess their performance in vivo ${ }^{733} 34$. 
Four novel rhesus macaque iPSC lines were generated using our previously published 6 reprogramming factor transposon ${ }^{23}$. The generated iPSC were pluripotent as indicated by pluripotency marker expression and teratoma formation. RT-PCR was performed with primer pairs able to discriminate between endogenous and exogenous origin of pluripotency-related genes. Robust expression of endogenous OCT4A, SOX2, $N A N O G$, and $c-M Y C$ transcripts was detected in the iPSCs as judged by comparison with Rh_ESCs. However, the analysis also revealed the presence of piggyBac-derived exogenous transcripts showing that the expression cassette was not fully silenced in iPSCs. The potency of the novel NHP-iPSC lines was also evaluated by teratoma formation. All lines analyzed formed teratomas. We were wondering whether the piggyBac cassette would be silenced upon iPSC differentiation over several weeks in the context of teratoma formation. Interestingly, most teratoma sections were negative for the pluripotency markers OCT4A, LIN28, and Nanog, for which we have established specific and sensitive immunohistochemical detection protocols ${ }^{29} 35$. We found only scattered clusters of relatively few positive cells. This shows downregulation of endogenous pluripotency genes, and, even more importantly, also downregulation of the reprogramming construct in the differentiated cells of the teratoma.

In order to analyze piggyBac silencing in the teratoma, we performed comparative methylation analysis of the two CAG promoters present in the piggyBac construct isolated from iPSCs and the teratomas. There was no clear difference between the methylation patterns found in iPSCs and in teratomas. However, between the two promoters, the CAG puromycin and the CAG reprogramming, we found differential methylation patterns. Surprisingly, the CAG reprogramming was highly methylated (in most samples close to 100\%) in comparison with the CAG puromycin promoter, which shows around $30 \%$ methylation in teratomas and around $20 \%$ in iPSCs. These findings were generally reproduced in all three independent iPSC lines. This suggests that the cells can discriminate between the two sequence-wise identical CAG promoters in the construct. Either the gene products regulate the methylation of the promoters or the broader DNA sequence context in which the actual CAG promoters are embedded. Targeted methylation has been previously described for this and other reprogramming approaches ${ }^{36}$. Moreover, the high methylation of the CAG reprogramming was not only found in the teratomas, but also in the iPSC. This indicates that methylation occurs mainly during fibroblast reprogramming and culture of the iPSCs, but not during differentiation, as we initially hypothesized. Constitutive reprogramming factor expression could lead to failed differentiation and tumorigenicity 3738 . However, in our experiments, we observed mainly differentiated cells within the teratomas. On the other hand, and more importantly, also the iPSC line after deletion of the piggyBac cassette (see below) shows some remaining OCT4Apositive cells in the teratoma indicating that not (only) the exogenous OCT4A expression was still detectable, but (at least also) the endogenous variant.

The piggyBac transposon can be removed from the genome of the cells without leaving a footprint 272839 . Even though the teratoma demonstrated silencing of the exogenous 
reprogramming factors in most of the cells, this would not be a safe condition regarding cell transplantation, and exogenous genetic material present in the iPSC genome is not acceptable for transplantation purposes. In order to generate transgenefree macaque iPSC, we exemplarily excised the reprogramming transposon from the genome of one iPSC line. The resulting transgene-free clone was then fully recharacterized. Pluripotency factor expression and potency of the cell line remained unchanged after transposon removal. In addition, karyotyping showed that the excision-ligation process did not generate any detectable chromosomal abnormalities. We are of course aware of reprogramming approaches based on non-integrating vectors like Sendai viruses and episomes. However, our own data (Chapter 2) show that rhesus monkey iPSC generation is significantly less efficient than human iPSC generation. Considering this, the integrating, yet reversible piggyBac approach for NHP-iPSC generation is still useful because of its robustness. In fact, piggyBac-based reprogramming proved useful for the generation of marmoset monkey (Debowski et al. 2015$)^{23}$, baboon (Rodriguez-Polo et al. 2019) ${ }^{30}$ and rhesus monkey (present study) iPSCs. From the latter two species, we obtained iPSCs also from adult and aged animals.

We adapted two of the generated NHP-iPSC to feeder-free conditions. Feeder-free culture is essential to obtain pure cell populations without the presence of (feeder) cells from other species. Furthermore, this allows up-scaled production and facilitates more efficient use of biotechnological tools, like CRISPR/Cas9 ${ }^{4041}$. The cell lines remained undifferentiated in the new conditions. Importantly, also the transgene-free iPSC line was cultured feeder-free. Hence, feeder-free and transgene-free culture of piggyBacderived rhesus iPSCs is possible.

Genome editing and single-cell cloning of PSC is challenging due to the low efficiency of the process and stress-induced death of the stem cells forced to single-cell separation 2425 . Fine-tuning of the protocols developed for human ${ }^{25} 42$ and mouse PSC ${ }^{43}$ was required in order to generate a robust protocol for rhesus macaque PSCs ${ }^{44}$. We have demonstrated that accutase and versene are more suitable dissociation reagents for the generation of PSC single-cell suspensions than TrypLE. Furthermore, we tested different compounds and conditions to increase the number of surviving single cells / clones after sorting. ROCK inhibitor (PSF) shows a beneficial effect in single-cell survival in the two lines analyzed, while hypoxia and Activin A showed no effect on the cloning efficiency in our experimental setup. Taking these results together, we developed a robust single-cell cloning protocol for rhesus PSC. Finally, we used a piggyBac vector containing eCas9-GFP plus guide RNAs to target two clinically relevant loci in the TTN gene. The piggyBac transposon combining all required components of the CRISPR/Cas9 editing machinery in one vector in combination with the fine-tuned protocol for single-cell cloning allowed us to reach very high efficiency in the generation of mutated clones, e.g., over $85 \%$ for the C-terminal TTN mutation. This genome editing approach is based on gDNA repair after targeting via non- 
homologous end joining (NHEJ). It is important to consider that this approach, even though being efficient, is imprecise.

Altogether, we demonstrated the suitability of the piggyBac system to reprogram and gene edit rhesus macaque iPSCs. We developed an efficient platform to evaluate CRISPR/Cas based genome editing approaches in PSCs before in vivo application. In combination with the new protocol for clonal expansion of gene-edited rhesus PSCs, this represents a useful in vitro screening platform for gene editing and thereby contributes to the 3Rs (reduce, replace, and refine) in animal experimentation. Differential methylation of the two identical CAG promoters present in the vector was encountered, with high methylation of the CAG reprogramming and low methylation of the CAG puromycin promoter. This may suggest a specific methylation response to the gene product controlled by the respective promoters. Finally, we have demonstrated removal of the reprogramming transposon by re-expression of the transposase, resulting in transgene-free iPSCs cultured under feeder-free conditions.

\section{References (Chapter 3)}

1. Firoz, C. K. et al. An overview on the correlation of neurological disorders with cardiovascular disease. Saudi J. Biol. Sci. 22, 19-23 (2015).

2. German, D., Mitalipov, S., Mishra, A. \& Kaul, S. Therapeutic Genome Editing in Cardiovascular Diseases. JACC Basic Transl Sci. 4, 122-131 (2019).

3. Dokken, B. B. The Pathophysiology of Cardiovascular Disease and Diabetes: Beyond Blood Pressure and Lipids. Diabetes Spectr. 21, 160-165 (2008).

4. Kathiresan, S. \& Srivastava, D. Genetics of Human Cardiovascular Disease. Cell. 148, 1242-1257 (2012).

5. Harding, J. D. Nonhuman Primates and Translational Research: Progress , Opportunities , and Challenges. ILAR 58, 141-150 (2017).

6. Boroviak, T. et al. Single cell transcriptome analysis of human, marmoset and mouse embryos reveals common and divergent features of preimplantation development. Development 145, 1-18 (2018).

7. Yoshimatsu, S. et al. Robust and efficient knock-in in embryonic stem cells and early- stage embryos of the common marmoset using the CRISPR-Cas9 system. Sci. Rep. 9, 1-12 (2019). 
8. Leong, X., Ng, C. \& Jaarin, K. Animal Models in Cardiovascular Research: Hypertension and Atherosclerosis. Biomed Res Int. 2015, 1-11 (2015).

9. Sasaki, E. et al. Generation of transgenic non-human primates with germline transmission. Nature 459, 523-527 (2009).

10. Cox, L. A. et al. Nonhuman Primates and Translational Research Cardiovascular Disease. ILAR J. 58, 235-250 (2017).

11. Behr, R. Primate biologics research at a crossroads.Potential of Genetically Modified Nonhuman Primate Models for Biomedicine. (Waxmann, 2015).

12. Yun, J., Ahn, J. \& Kang, B. Modeling Parkinson's disease in the common marmoset ( Callithrix jacchus ): overview of models , methods, and animal care. Lab Anim Res 6055, 155-165 (2015).

13. Sato, K. \& Sasaki, E. Genetic engineering in nonhuman primates for human disease modeling. J. Hum. Genet. 63, 125-131 (2017).

14. Shrock, E. \& Güell, M. Chapter six: CRISPR in Animals and Animal Models. Progress in Molecular Biology and Translational Science 152, (Elsevier Inc., 2017).

15. Wolfgang, M. J. et al. Rhesus monkey placental transgene expression after lentiviral gene transfer into preimplantation embryos. PNAS 98, 10728-10732 (2001).

16. Shi, L. et al. Transgenic rhesus monkeys carrying the human MCPH1 gene copies show human-like neoteny of brain development. Natl. Sci. Rev. 6, 480-493 (2019).

17. Chan, A., Chong, K., Martinovich, C., Simerly, C. \& Schatten, G. Transgenic Monkeys Produced by Retroviral Gene Transfer into Mature Oocytes. Science . 291, 309-312 (2001).

18. Yang, S. et al. Towards a transgenic model of Huntington's disease in a nonhuman primate. Nature 453, 921-924 (2009).

19. Zhang, X., Pang, W., Hu, X., Li, J. \& Yao, Y. Experimental primates and nonhuman primate ( NHP ) models of human diseases in China : current status and progress. Zool. Res. 35, 447-464 (2014).

20. Xuan Ho, B., Jia Hui Loh, S., Khiong Chan, W. \& Seng Soh, B. In Vivo Genome Editing as a Therapeutic Approach. Int. J. Mol. Sci. 19, 1-19 (2018). 
21. Takahashi, K. \& Yamanaka, S. Induction of Pluripotent Stem Cells from Mouse Embryonic and Adult Fibroblast Cultures by Defined Factors. Cell. 126, 663-676 (2006).

22. Takahashi, K. et al. Induction of Pluripotent Stem Cells from Adult Human Fibroblasts by Defined Factors. Cell. 131, 861-872 (2007).

23. Debowski, K. et al. Non-viral generation of marmoset monkey iPS cells by a sixfactor-in-one-vector approach. PLoS One 10, 1-21 (2015).

24. Wang, G. et al. Efficient, footprint-free human iPSC genome editing by consolidation of Cas9 / CRISPR and piggyBac technologies. Nat. Protoc. 12, 88103 (2016).

25. Chen, Y. \& Pruett-miller, S. M. Improving single-cell cloning work flow for gene editing in human pluripotent stem cells. Stem Cell Res. 31, 186-192 (2018).

26. Woltjen, K. et al. piggyBac transposition reprograms fibroblasts to induced pluripotent stem cells. Nature 458, 766-771 (2009).

27. Patel, M. \& Yang, S. Advances in Reprogramming Somatic Cells to Induced Pluripotent Stem Cells. Stem Cell Rev. 6, 367-380 (2010).

28. Malik N \& Mahendra S. R. A Review of the Methods for Human iPSC Derivation. Methods Mol Biol. 997, 23-33 (2013).

29. Aeckerle, N., Drummer, C., Debowski, K., Viebahn, C. \& Behr, R. Primordial germ cell development in the marmoset monkey as revealed by pluripotency factor expression: suggestion of a novel model of embryonic germ cell translocation. Mol. Hum. Reprod. 21, 66-80 (2015).

30. Rodriguez-polo, I. et al. Baboon induced pluripotent stem cell generation by piggyBac transposition of reprogramming factors. Primate Biol., 6, 75-86 (2019).

31. Eildermann, K. et al. Developmental expression of the pluripotency factor sal-like protein 4 in the monkey, human and mouse testis: Restriction to premeiotic germ cells. Cells Tissues Organs 196, 206-220 (2012).

32. Hill, J. T. et al. Poly Peak Parser: Method and software for identification of unknown indels using Sanger Sequencing of PCR products. Dev Dyn. 243, 16321636 (2015).

33. Debowski, K. et al. The transcriptomes of novel marmoset monkey embryonic stem cell lines reflect distinct genomic features. Sci. Rep. 6, 1-13 (2016). 
34. Hong, S. G. et al. Rhesus iPSC Safe Harbor Gene-Editing Platform for Stable Expression of Transgenes in Differentiated Cells of All Germ Layers. Mol. Ther. 25, 44-53 (2017).

35. Wolff, E., Suplicki, M. M. \& Behr, R. Primordial germ cells do not migrate along nerve fibres in marmoset monkey and mouse embryos. Reproduction 157, 101109 (2019).

36. Troyanovsky, B., Bitko, V., Pastukh, V., Fouty, B. \& Solodushko, V. The Functionality of Minimal PiggyBac Transposons in Mammalian Cells. Mol. Ther. Acids 5, (2016).

37. Kang, X. et al. Effects of Integrating and Non-Integrating Reprogramming Methods on Copy Number Variation and Genomic Stability of Human Induced Pluripotent Stem Cells. PLoS One 10, 1-12 (2015).

38. Medvedev, S. P., Shevchenko, A. I. \& Zakian, S. M. Induced Pluripotent Stem Cells : Problems and Advantages when Applying them in Regenerative Medicine. Acta Naturae 2, 18-27 (2010).

39. Woltjen, K., Hämäläinen, R., Mark Kibschull, Mileikovsky, M. \& Nagy, A. Transgene-free production of pluripotent stem cells using piggyBac transposons. Methods Mol Biol. 767, 87-103 (2011).

40. Sosa, E. et al. An integration-free, virus-free rhesus macaque induced pluripotent stem cell line (riPSC89) from embryonic fibroblasts. Stem Cell Res. 21, 5-8 (2017).

41. Zhang, X., Cao, H., Bai, S., Huo, W. \& Ma, Y. Differentiation and characterization of rhesus monkey atrial and ventricular cardiomyocytes from induced pluripotent stem cells. Stem Cell Res. 20, 21-29 (2017).

42. Tidball, A. M. et al. Rapid Generation of Human Genetic Loss-of-Function iPSC Lines by Simultaneous Reprogramming and Gene Editing. Stem Cell Reports 9 , 725-731 (2017).

43. Mehravar, M., Shirazi, A., Mehrazar, M. M., Nazari, M. \& Salimi, M. Efficient Production of Biallelic RAG1 Knockout Mouse Embryonic Stem Cell Using CRISPR / Cas9. Iran. J Biotech 17, 45-53 (2019).

44. Bressan, R. B. et al. Efficient CRISPR / Cas9-assisted gene targeting enables rapid and precise genetic manipulation of mammalian neural stem cells. Development 144, 635-648 (2017). 


\section{Supplementary material (Chapter 3)}

\begin{tabular}{ccc} 
& cDNA & Protein $(\%)$ \\
\hline SOX2 & $93,96 \%$ & $99,68 \%$ \\
OCT4 & $85,46 \%$ & $95,63 \%$ \\
KLF4 & $94,56 \%$ & $100,00 \%$ \\
\hline LIN28 & $96,51 \%$ & $99,04 \%$ \\
c-MYC & $94,65 \%$ & $97,95 \%$ \\
NANOG & $86,06 \%$ & $94,44 \%$
\end{tabular}

Table 1: Percentage of similarity for the reprogramming factors used between marmoset and rhesus macaque. Comparison on cDNA and protein level. Marmoset vs. rhesus macaque alignment for SOX2 (ENSCJAG00000008401/ENSMMUG00000046147), OCT4A (ENSCJAG00000019789/ENSMMUG00000015688), 
Chapter 3: A piggyBac-based platform for genome editing and clonal rhesus macaque iPSC line derivation

\begin{tabular}{|c|c|c|c|c|c|}
\hline & Oligo name & Sequence & $\operatorname{Tm}\left({ }^{\circ} \mathrm{C}\right)$ & ong (se & Amp (bp) \\
\hline \multirow{12}{*}{ RT-PCR } & G302_LIN28-NANOG_fw & AGCCATATGGTAGCCTCATGTCC & 62 & 55 & 811 \\
\hline & G70_LIN28-NANOG_rev & GGTTGCTCCAGGTTGAATTGC & & & \\
\hline & G2212_OCT4 endo_fw & GAGAAGGAGAAGCTGGAGCAA & 52.9 & 60 & 841 \\
\hline & G2213_OCT4 endo_rev & ACATCCTTCTCGAGCCCAA & & & \\
\hline & G2008_SOX2 endo_fw & GGTAGGAGCTTTGCAGGAAGT & 61 & 30 & 428 \\
\hline & G2009_SOX2 endo_rev & CCAACGATGTCAACCTGCATG & & & \\
\hline & G2237_NANOGendo_fw & CAGAGATACCTCAGCCTCCAG & 54.4 & 35 & 562 \\
\hline & G2238_NANOGendo_rev & CTTCAGGTTGCATGTTCGT & & & \\
\hline & G2010_cMYCendo_fw & CTGGTACTCCATGAGGAGACA & 61 & 60 & 715 \\
\hline & G2011_cMYCendo_rev & CTCAGCCAAGGTTGTGAGGTT & & & \\
\hline & G2204_beta actin & GGTAGTTTCGTGGATGCCACA & 61 & 30 & 379 \\
\hline & G2205_beta actin & GACCTGACTGACTACCTCATG & & & \\
\hline \multirow{12}{*}{ PiggyBac detection } & G1842_piggydetecF2Afw & GTGAAACAGACTTTGAATTTTGACC & 54 & 10 & 97 \\
\hline & G1843_piggydetecF2Arev & AATCCGAAGCCAGGTGTC & & & \\
\hline & G1844_piggydetecE2Afw & TACACATGAAGAGGCATTTTCAATG & 55 & 10 & 114 \\
\hline & G1845_piggydetecE2Arev & TGCTGAAGCTGACGTTGA & & & \\
\hline & G1846_piggydetecpAfw & TGCCACTCCCACTGTCCTTTCCTA & 57 & 10 & 116 \\
\hline & G1847_piggydetecpArev & CAАТСCTCССССТTGCTG & & & \\
\hline & G302_LIN28-NANOGfw & AGCCATATGGTAGCCTCATGTCC & 62 & 55 & 811 \\
\hline & G70_LIN28-NANOG_rev & GGTTGCTCCAGGTTGAATTGC & & & \\
\hline & G244_Pcag-SOx2fw & GGGGACGGCTGCCTTCGG & 60 & 20 & 361 \\
\hline & G395_Pcag-SOx2rev & CGGTCGGGGCTGTTCTTCTG & & & \\
\hline & G2204_beta actin & GGTAGTTTCGTGGATGCCACA & 61 & 30 & 379 \\
\hline & G2205_beta actin & GACCTGACTGACTACCTCATG & & & \\
\hline \multirow{4}{*}{ gRNAs } & G1806_TTN_N-terminusfw & CACCgGCTGACTACACCTTTGTGGC & & & \\
\hline & G1807_TTN_N-terminusrev & AAACGCCACAAAGGTGTAGTCAGCc & & & \\
\hline & G1822_TTN_C-terminusfw & CACCgGTTACTGCTTCCAATCGCCT & & & \\
\hline & G1823_TTN_C-terminusrev & AAACAGGCGATTGGAAGCAGTAACc & & & \\
\hline \multirow{4}{*}{ Clonal line analysis } & G2616_N-termfw & GCATGGTGGCACAGAGTTGT & 61 & 100 & 1861 \\
\hline & G2617_N-termrev & TAGACAGCTGCTAGGGACAC & & & \\
\hline & G2654_C-termfw & GCGACTCTGCCCAACTACAT & 61 & 60 & 828 \\
\hline & G2655_C-termrev & GCAGTGTTGGTGACTTCCTC & & & \\
\hline \multirow{10}{*}{ Methylation analysis } & G2656_CAG_Reprogfw & GGGATTTTTTTTGTTTTAAATTTGTG & 60 & 25 & 401 \\
\hline & G2657_CAG_Reprogrev & AАTTCCACCACACTAAACTAAT & & & \\
\hline & G2658_CAG_Purofw & GGGATTTTTTTTGTTTTAAATTTGTG & 64 & 35 & 575 \\
\hline & G2659_CAG_Purorev & TATCACССТСТСAАТАТАССТАТC & & & \\
\hline & G2660_CAGfw & GTAGTTATTGTTTTTTATGGTAA & 52 & 25 & 373 \\
\hline & G2661_CAGrev & TAATAAAACAACACAATAACCAACAC & & & \\
\hline & G2662_CAG_Inner_fw & GGGATTTTTTTTGTTTTAAATTTGTG & 53 & 29 & 333 \\
\hline & G2663_CAG_Inner_rev & TAATAAAACAACACAATAACCAACAC & & & \\
\hline & G2664_CAG_Inner_Seq_fw & CATAAACATAATTAACAAAAACTCT & & & \\
\hline & G2665_CAG_Inner_Seq_rev & ССССССССАТТТССТТ & & & \\
\hline
\end{tabular}

Table 2: Oligos used in this study. PCR conditions defined by annealing temperature (Tm), elongation (Elong), and amplicon (Amp). (*) 5'-Biotin. 
(A)
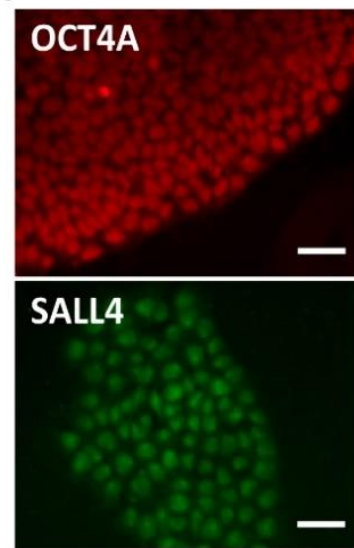

SOX2

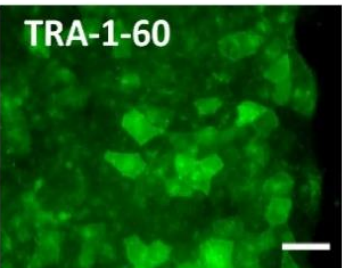

\section{TRA-1-81}
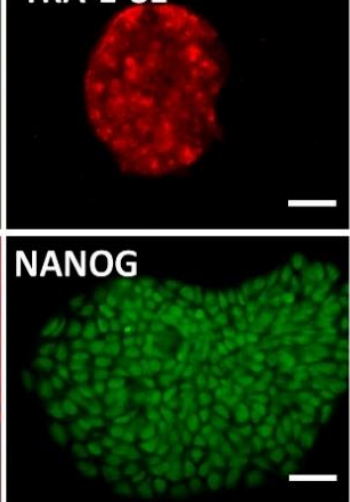

(B)

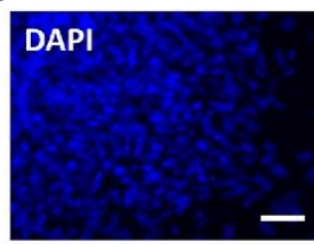

IgG Rabbit

DAPI

IgM Mouse

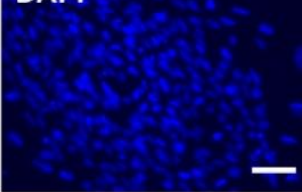

DAPI

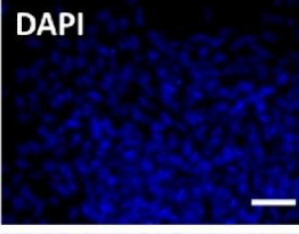

No primary $\mathrm{Ab}(\mathrm{A})$

DAPI

Suppl. Figure 1: Immunofluorescence staining of DPZ_iRhpb\#4 and negative/isotype controls. (A) Immunofluorescence staining of DPZ_iRhpb\#4. Detection of OCT4A, LIN28, TRA-1-60, SOX2, TRA1-81, and SALL4. OCT4A, LIN28, and SOX2 expression come from both endogenous and piggyBac expression. (Scale bar 20 $\mu \mathrm{m})$. (B) Immunofluorescence negative and isotype controls. Isotype controls.

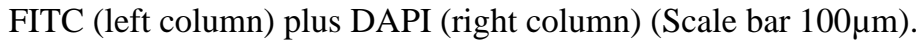

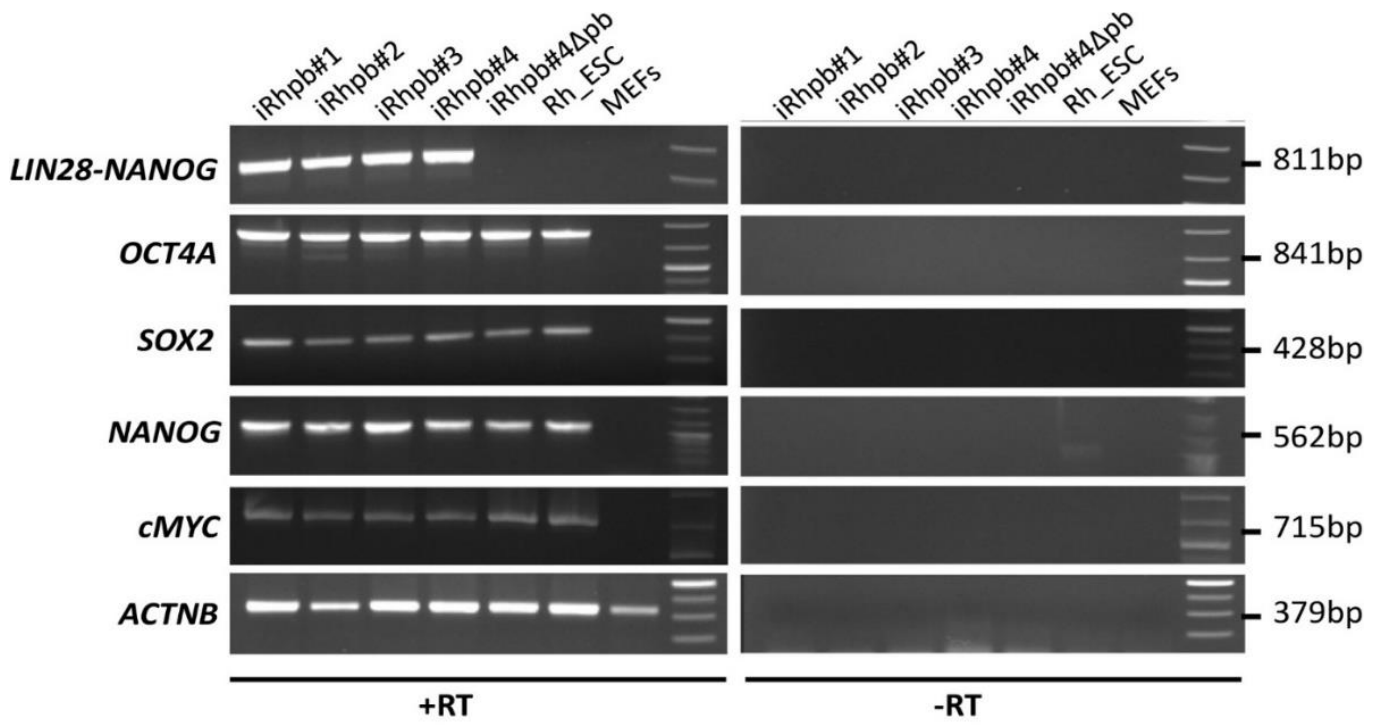


Suppl. Figure 2: Rhesus iPSC characterization by RT-PCR: Expression analysis of the four generated Rhesus iPSC lines (DPZ_iRhpb\#1-4), and transgene-free DPZ_iRhpb\#4 $\Delta \mathrm{pb}$. Rhesus embryonic stem cells (Rh_ESC) and MEFS were added as positive and negative controls, respectively. Primers for the RT-PCR were designed for the specific amplification of the endogenous pluripotency factors OCT4A, SOX2, NANOG, and c-MYC. LIN28-NANOG amplicon shows transposon expression in the transgenic lines and absence of exogenous transcript in DPZ $i$ Rhpb $\# 4 \triangle \mathrm{pb}$. Beta-actin expression $(A C T N B)$ was used as a housekeeping gene (marker $1 \mathrm{~kb}$ Plus DNA ladder, NEB).

(A)
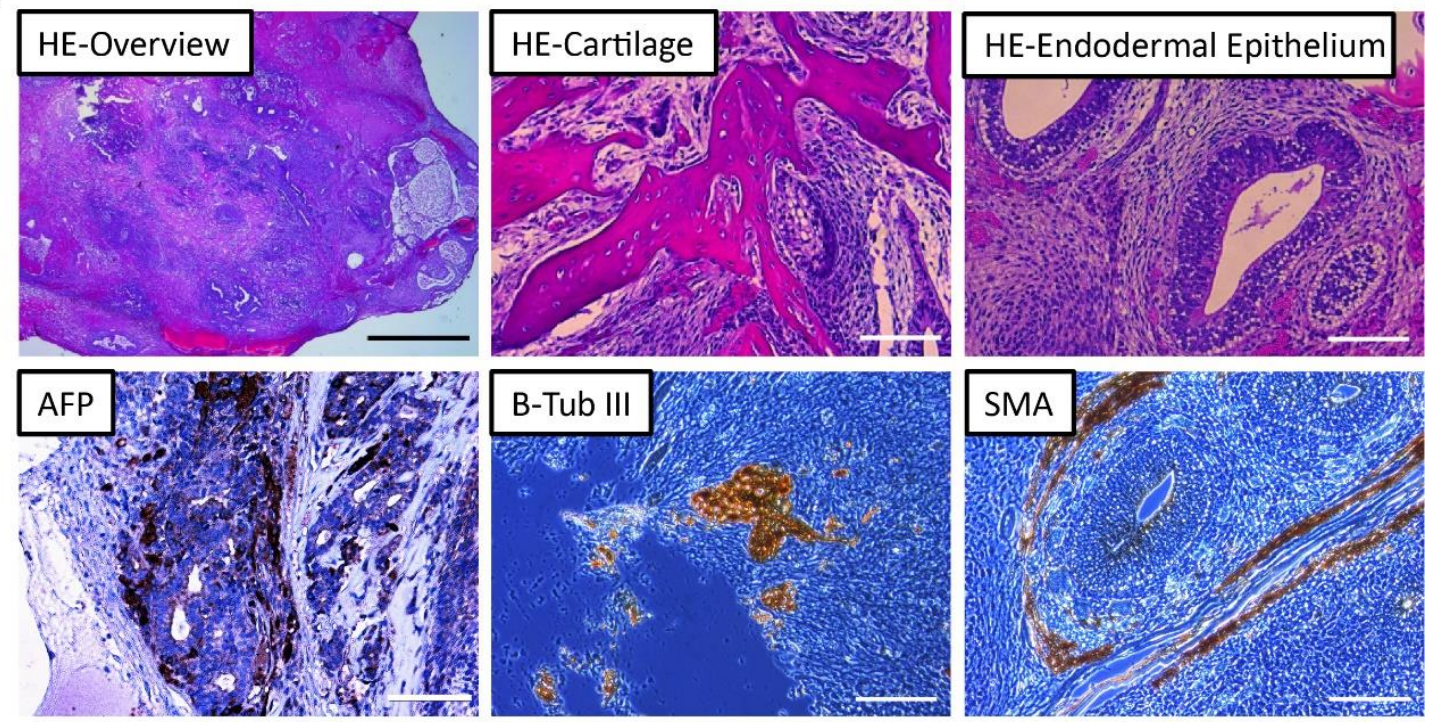

(B)
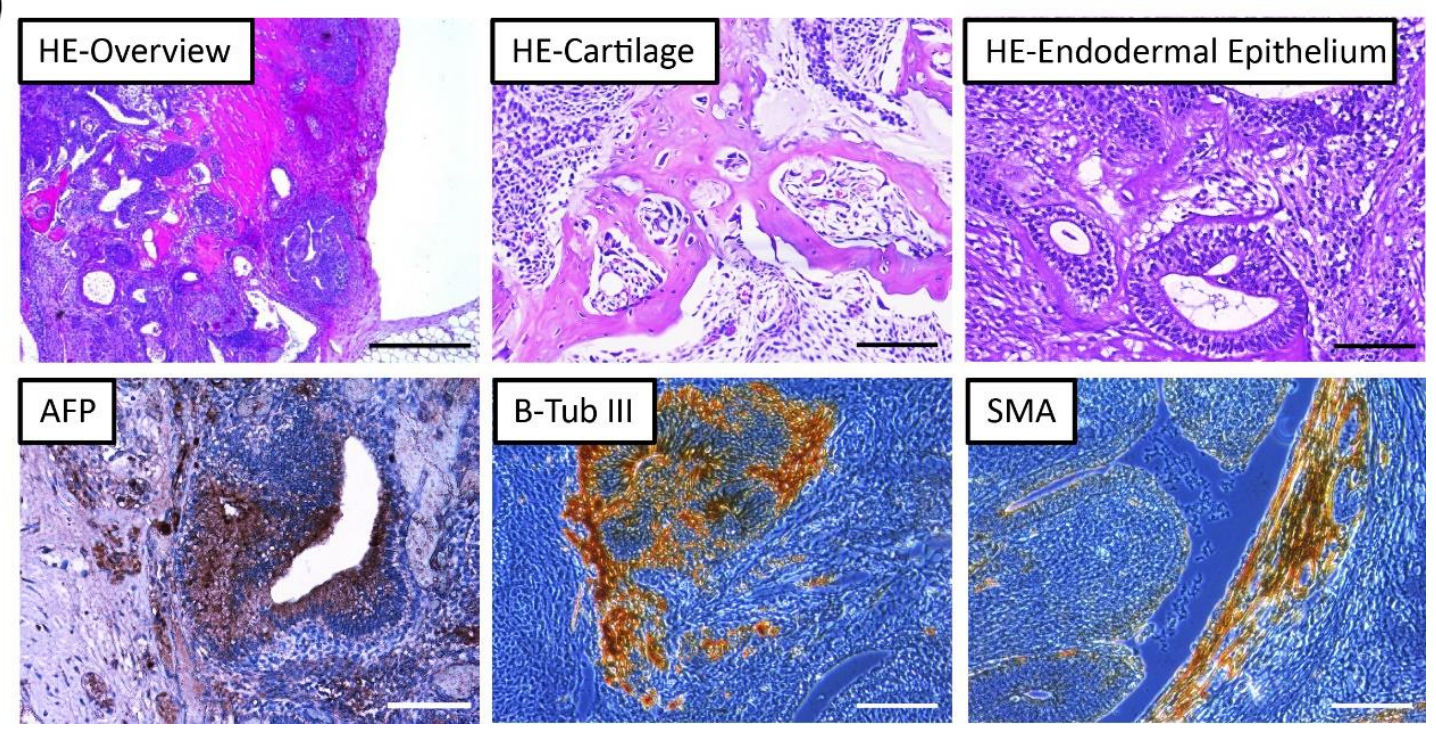

Suppl. Figure 3: Immunohistochemical analysis of DPZ_iRhpb\#1 (A) and DPZ_iRhpb\#3 (B) teratomas. Teratoma sections were stained for representative markers of the three germ layers: $\beta$ - tubulin III, smooth muscle actin (SMA), and Alpha-1-Fetoprotein (AFP). Complementarily HE staining was performed to identify specific cytological features of representative tissues. $\beta$ - Tubulin III indicate 
mesodermal differentiation. Smooth muscle actin and AFP staining show ectodermal and endodermal differentiation, respectively. Presence of cartilage indicates mesoderm. Moreover, gut endodermal epithelium support AFP staining, showing also the presence of endodermal tissue (Scale bar 100 $\mu \mathrm{m}$ ).

\section{(A)}
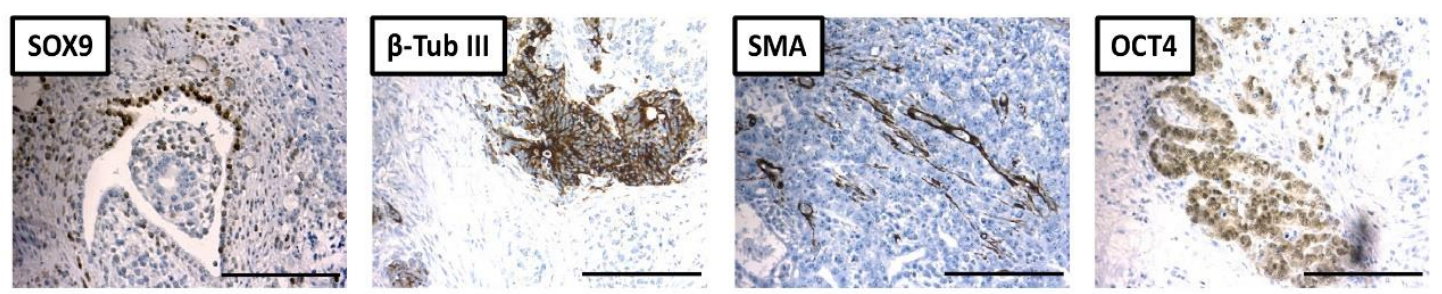

(B)
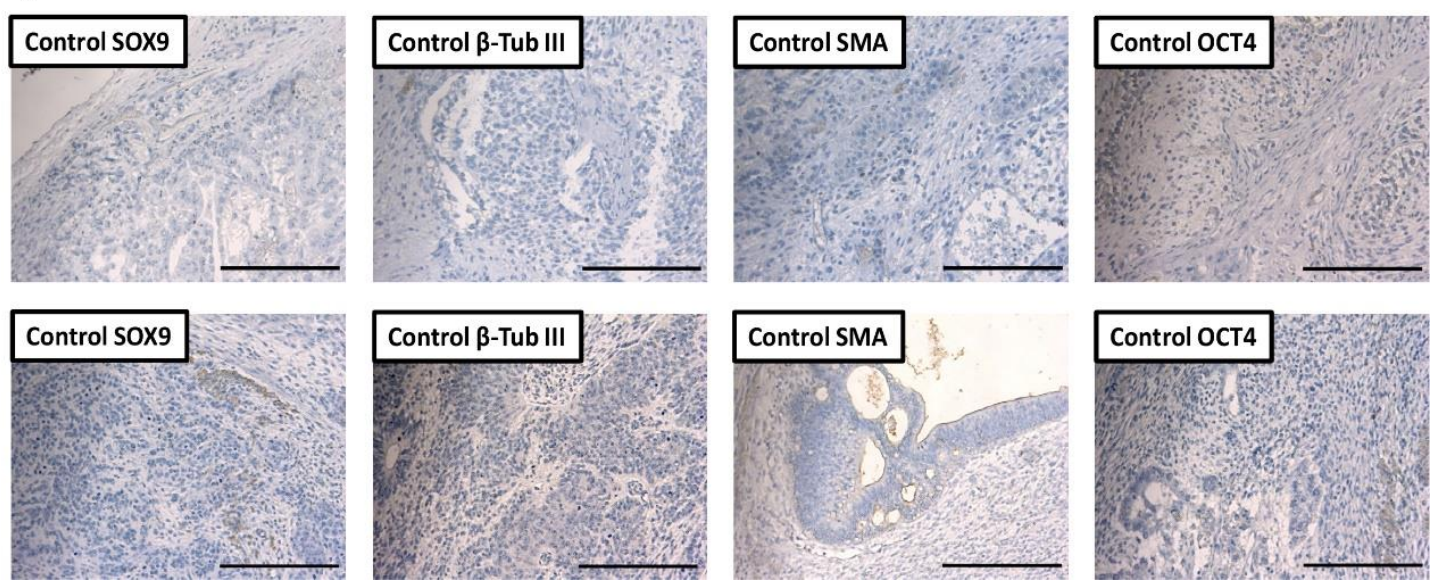

Suppl. Figure 4: Immunohistochemical analysis of DPZ_iRhpb\#4 teratoma and isogenic controls (teratomas DPZ_iRhpb\#1 and DPZ_iRhpb\#3). (A) Teratoma sections from DPZ_iRhpb\#4 stained for SOX9, $\beta$ - tubulin III, smooth muscle actin (SMA), and OCT4A. $\beta$ - Tubulin III staining indicate mesodermal differentiation. Smooth muscle actin show ectodermal differentiation and AFP staining

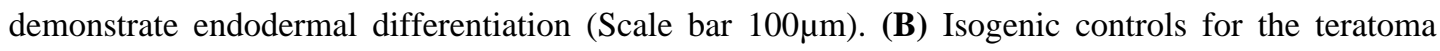
staining. Upper row controls for DPZ_iRhpb\#1, lower row controls for DPZ_iRhpb\#3. Isogenic controls for the markers SOX9, $\beta$ - tubulin III, SMA, and OCT4A (Scale bar 100 $\mu \mathrm{m}$ ). 
(A)

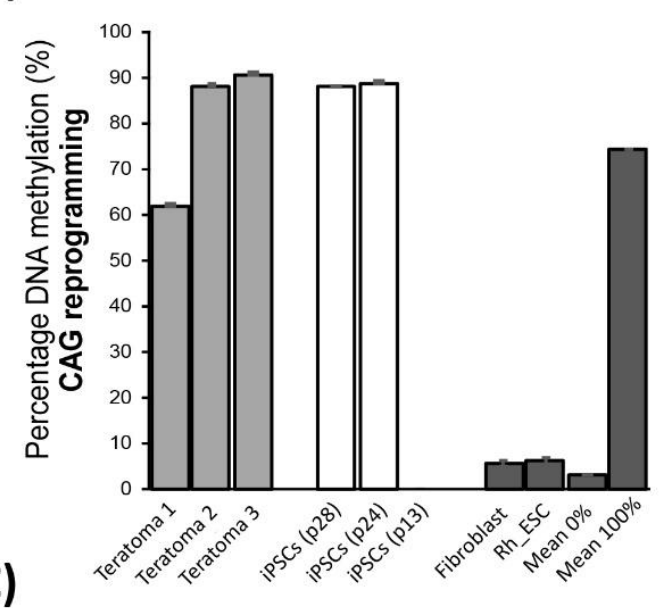

(C)

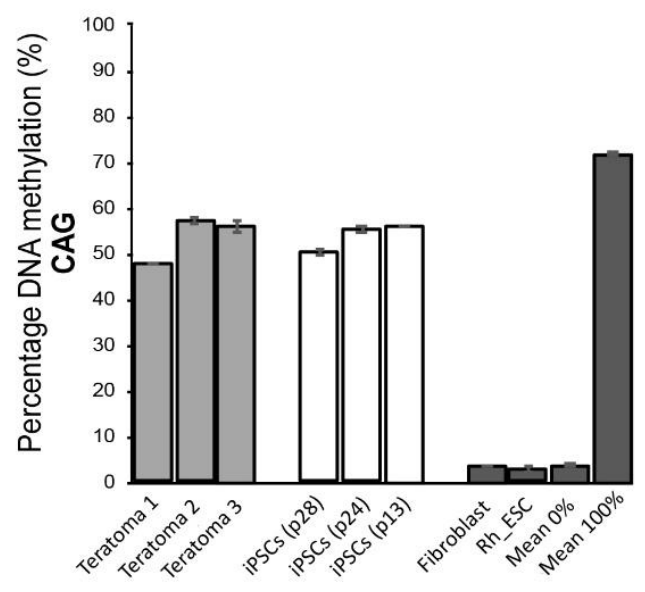

(B)

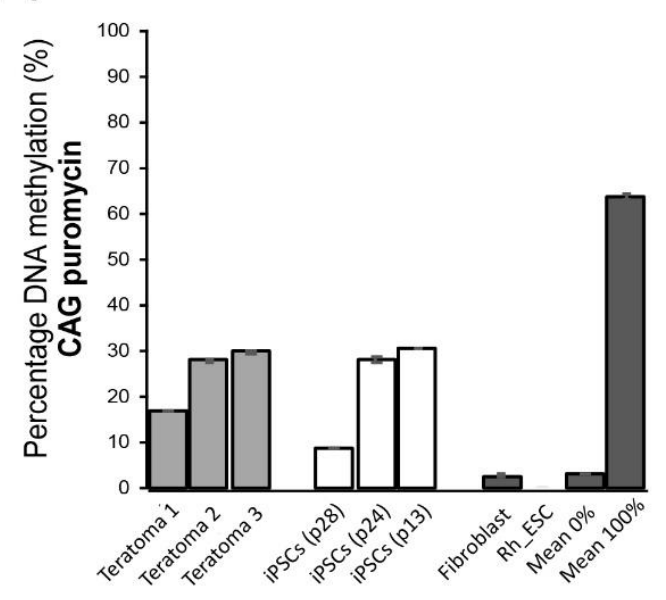

Suppl. Figure 5: Methylation analysis of the reprogramming construct using sequencing primer, S1. Methylation analysis of three teratomas (teratomas 1-3), and three iPSC. Two samples were included as negative control: Fibroblast and Rh_ESC. Internal controls are represented (Mean 0\% and Mean $100 \%$ ) (Mean \pm SD). (A) CAG reprogramming methylation analysis. (B) CAG puromycin methylation analysis. (C) CAG (including both promoters) methylation analysis. 
(A)
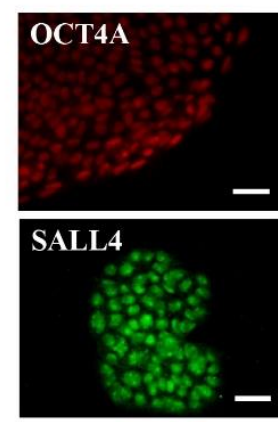

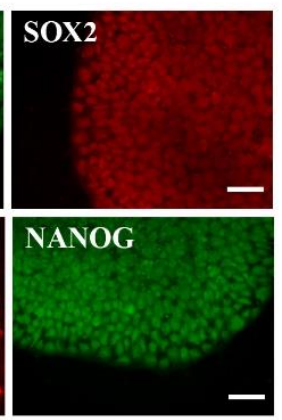

(B)
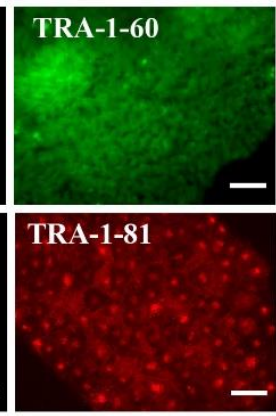

(C)
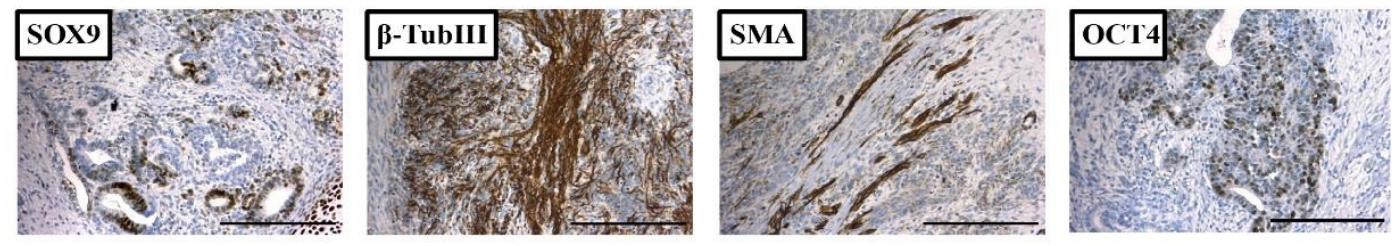

Suppl. Figure 6: $D P Z \_i R h p b \# 4 \Delta p b$, characterization. (A) Immunofluorescence staining. Detection of OCT4A, LIN28, TRA-1-60, SOX2, TRA-1-81 and, SALL4. In DPZ_iRhpb\#4Dpb the piggyBac reprogramming construct has been removed, and consequently, the expression of all pluripotency

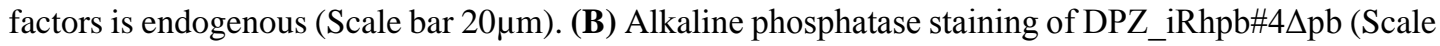
bar $100 \mu \mathrm{m})$. (C) Immunohistochemical analysis of DPZ $i R h p b \# 4 \Delta \mathrm{pb}$ teratoma. Teratoma sections were stained for representative markers of the three germ layers SOX9, $\beta$ - tubulin III, smooth muscle actin (SMA), and OCT4A to show cells that remain undifferentiated in the tumor (Scale bar 100 $\mu \mathrm{m}$ ).
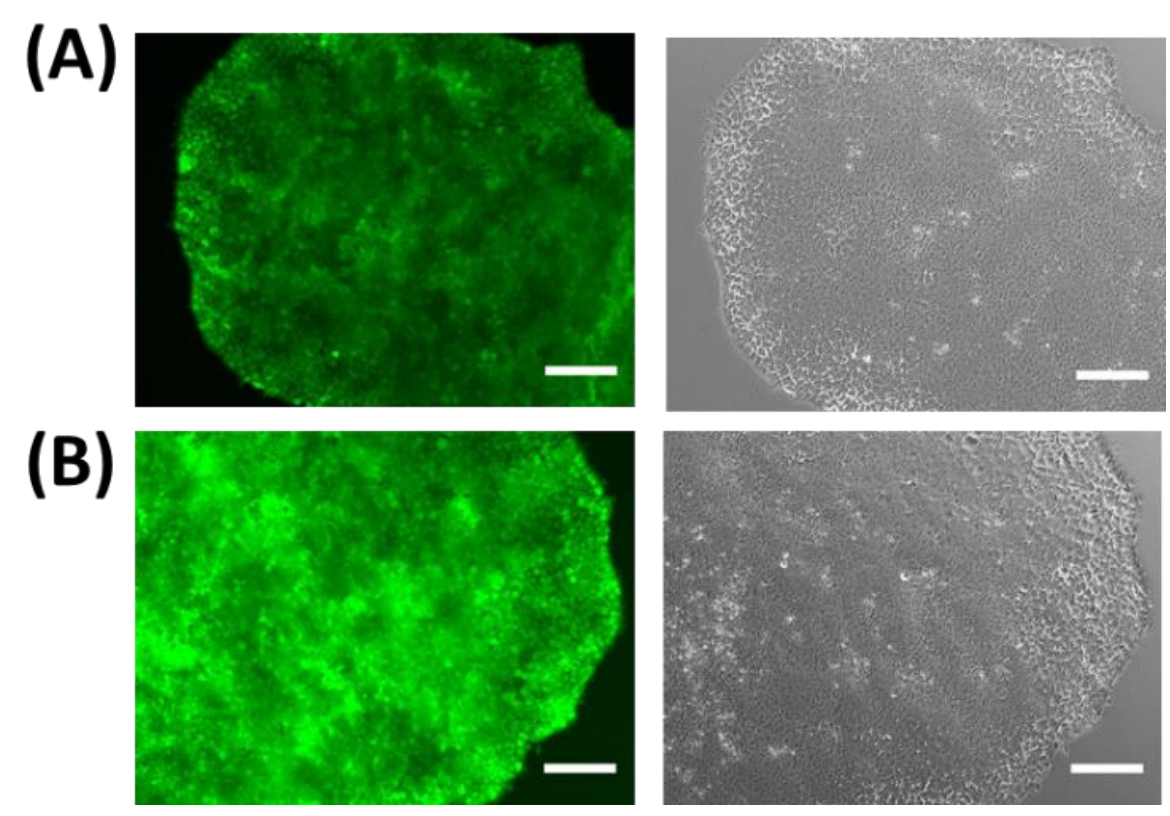

Suppl. Figure 7: Isogenic controls generated transfecting DPZ_iRhpb\#4 (A) and Rh_ESC (B) with pCAG-eCas9-GFP-U6-gRNA-Neo. The lines constitutively express eCas9 and GFP but no gRNA (Scale bar $100 \mu \mathrm{m})$. 


\section{Chapter 4: An iPSC-based preselection platform for disease-inducing genetic modifications in non- human primates}

Author contribution statement

IRP, and RB conceived and designed the experiments. IRP, IG, YT and DU performed the experiments. IRP, IG, YT, DU, and RB analyzed the data. IRP and RB wrote the manuscript with contributions from all coauthors.

\begin{tabular}{|l|l|}
\hline \multicolumn{2}{|c|}{ Author contribution statement } \\
\hline \multicolumn{2}{|c|}{$\begin{array}{c}\text { Chapter 4: An iPSC-based preselection platform for disease-inducing } \\
\text { genetic modifications in non-human primates }\end{array}$} \\
\hline All figures & $\begin{array}{l}\text { IRP and IG, YT, DU (during their master projects under the supervision of IRP) } \\
\text { performed the experiments }\end{array}$ \\
\hline Experiment design & IRP and RB \\
\hline Manuscript writing & IRP and RB, with the contribution from all authors \\
\hline
\end{tabular}




\title{
An iPSC-based preselection platform for disease-inducing genetic modifications in non-human primates
}

\author{
Ignacio Rodriguez-Polo ${ }^{1,2}$, Iga Grządzielewska ${ }^{3}$, Julia Tereshchenko ${ }^{3}$, Daniel Urrutia
} Cabrera ${ }^{1}$, Rüdiger Behr ${ }^{1,2}$

${ }^{1}$ Research Platform Degenerative Diseases, German Primate Center - Leibniz Institute for Primate Research, Kellnerweg 4, 37077 Göttingen, Germany

${ }^{2}$ German Center for Cardiovascular Research (DZHK), Partner site, Göttingen, Germany

${ }^{3}$ Max Planck molecular Biology program (M.Sc./Ph.D.), Justus-von-Liebig-Weg 11, 37077 Göttingen, Germany

\begin{abstract}
Cardiovascular diseases (CVDs) are a heterogeneous group of pathologies with high incidence in the population. Even though these diseases have a complex etiology, most of them have a genetic origin. CVD models will help to understand the cause and progression of the diseases. This becomes particularly relevant when looking at the economic and social burden caused by CVDs. To recapitulate CVD phenotypes, utilization of animal models sharing the complexity of the human cardiovascular system is necessary. Non-human primates (NHP), due to their close phylogenetic relationship with humans, meet all requirements of translational animal models. In recent years, genome editing has gained in sophistication, allowing human genotypes to be mimicked in NHP. However, this process is still laborious and time-consuming. Here, we combined previously published and novel approaches to generate genomically modified iPSC from NHP. Our workflow allows the generation of a species-specific iPSC based platform for in vitro evaluation of genome editing approaches. This becomes particularly relevant when thinking about genetically modified NHP in vivo models. Our study includes two Old World and one New World monkey species, and the iPSC lines from all of them, have been generated under equivalent conditions. Furthermore, differentiation of the transgenic iPSC into functional cardiomyocytes may allow at least partial in vitro prediction of the in vivo phenotype. Furthermore, we have tuned a CRISPR based approach to induce point INDEL and fragment deletion mutations in NHP-iPSC. Finally, we have empirically developed an approach to the treatment of monogenic CVDs. We hypothesized that the extraordinarily high percentage of clinically relevant symmetric exons in the giant Titin gene could be used to develop CRISPR based reframing strategies to treat dilated cardiomyopathy (DCM) in patients with truncating titin variants. In summary, we have developed an iPSC-based in vitro workflow for the refinement of genome editing approaches before they are applied in vivo in NHP.
\end{abstract}




\section{Introduction}

Genetically modified animals are animals which genome has been modified by the human using biotechnological tools ${ }^{12}$. Such animals have revolutionized many areas impacting on fields like agricultural industry, medicine, ecology, and biomedical research ${ }^{34}$. The appearance of novel and highly efficient genome editing tools has facilitated the generation of additional animals species with highly sophisticated genetic modifications ${ }^{56}$.

Generation of disease-specific genetically modified animals is especially advantageous when considering the limitations of conventional disease induction methods (e.g., drug- or surgically-induced disease). Longitudinal studies can be achieved since the mutation is generally passed on to the offspring. This allows animal strain establishment and decreases experimental variation amongst different animals ${ }^{5}$.

Historically, genetic modification of rodents has been used in biomedical research, due to the easy handling of these animals, together with in-depth knowledge about their reproductive biology and the early availability of chimera-competent embryonic stem cells in mice ${ }^{37}$. Other animal models, due to their species-specific characteristics, present more difficulties regarding the application of these technologies. In cardiovascular research, it is important to work with a model resembling human organ size and metabolism, general physiology, diet, and life span. While rodents fail to show these attributes, non-human primates (NHP) pose an interesting model candidates, due to their close phylogenetic proximity to human ${ }^{86}$. Therefore, the generation of genetically modified NHP in order to model human diseases is one of the major research interests 5 .

For the induction of selected mutations of the NHP genome, it is essential to adapt genome editing protocols developed in other species to $\mathrm{NHP}^{9}$. This needs to be done as precisely as possible in order to prevent any unintended mutations. Additionally, genetic modification of NHP entails some difficulties not found in other organisms ${ }^{10}$. Despite the usage of refined genome editing methodologies like CRISPR/Cas, robust protocols that can be applied to these animal models are still required. Furthermore, the generation of a genetically modified NHP is a high-risk project, due to the long duration, high costs, and the experimental state of reproductive technologies in these species ${ }^{4}$.

The general work-flow for genetically modified animal generation is well defined and based on existing rodent models. Starting with the identification of a clinically relevant mutation in patients, the mutation is translated to the model's genotype in silico. Once the modification is fully defined, genome editing tools (e.g. CRISPR) are designed to target the location of interest. The gene modification components are then introduced into an preimplantation embryo or a zygote and then retransferred into a foster mother. After gestation, the offspring is analysed genotypically and phenotypically 56 . 
To pave the way towards the generation of a precisely genetically modified NHP, we and others believe that it is imperative to include an additional pre-screening step. Before targeting the embryo an evaluation of the genome-editing tool is required in order to prevent the generation NHP with unintended genetic modifications. Additionally, an initial in vitro evaluation of the modification is necessary due to the ethical controversies associated with NHP experimentation. For the evaluation of a genome editing approach, it is necessary to use species-specific cells with a full genetic homology to the model species. NHP pluripotent stem cells (PSC) offer an excellent opportunity for this purpose. Besides the species-specific origin, they share molecular characteristics with the pluripotent stem cells of the early embryo ${ }^{11} 1213$. Additionally, these cells can be differentiated into cells of all three germ layers like cardiomyocytes and neurons, allowing a preliminary analysis of the in vivo phenotype.

Generation of genetically modified NHP will help to understand pathologies like dilated cardiomyopathy (DCM), a disease that due to its complex etiology makes it challenging to understand its physiopathology. DCM is a disease that affects approximately 1 out of 2,500 persons, and has been found in the last years showing accelerated frequency ${ }^{14}{ }^{16}$. DCM is diagnosed according to two factors: 1- left ventricular enlargement and 2- systolic dysfunction recognizable by reduction of myocardial contraction force ${ }^{16}$. Approximately $30 \%$ of the cases of DCM are inherited. Mutations in more than 30 genes can lead to DCM, making it a highly complex and heterogeneous disease. $25 \%$ of familiar and $18 \%$ of sporadic cases can be accounted to mutations of the sarcomeric protein Titin (TTN) (nonsense, frameshift or canonical splice site) 16171819 . Frameshift mutations in TTN alter the reading frame, generating truncated versions of the protein (tvTTN) that lead to premature termination of translation ${ }^{2021}$.

To the present, therapies for DCM are limited, given the limited self-renewal capabilities of the cardiac muscle. In severe cases, heart transplantation is the only option. However, transplantations also represent a bottleneck due to limited donor organ availability. In contrast, promising approaches for treating a significant subset of DCM patients are genome editing technologies to reset the reading frame in patients with tvTTN ${ }^{15} 20$.

A skip or deletion of mutated exons leading to a shift of the open reading frame of a gene has already been considered as a potential strategy to treat DCM and other cardiovascular diseases (CVDs) ${ }^{22} 23$. In the past years, a variety of endonuclease-based experimental treatments were tested and established to overcome frameshift mutations in sarcomeric proteins 242526 . These approaches can be summarized in four major groups (i) controlled splicing of mutated exons by inducing indel mutations in the splice acceptor-donor site (ii) full fragment/exon removal (iii) exon reframing via targeted frameshift and (iv) exon knock-in 2627 . It is crucial to explore novel treatments for DCM using the mentioned approaches. However, these genome editing approaches require, as the generation of genetically modified primates, validation. For this purpose, iPSC in combination with 
predictive animal models will play a key role in the safe translation of such kind of novel gene-modifying therapies to the clinics.

In the present study, we have refined our previously developed primate cell reprogramming and iPSC maintenance protocol (Chapter 2) and extended it to the marmoset monkey, a new world monkey species. Using the new work-flow, we are able to generate transgeneand feeder-free marmoset monkey iPSC meeting the high quasi clinical-grade standards for human. Additionally, we have developed a robust protocol to apply CRISPR/Cas technologies to the generated iPSC from all species with high efficiency. Altogether, we aim to establish a platform for the testing and evaluation of NHP genome editing approaches before their application in vivo, i.e. the generation of a genetically modified NHP. Finally aiming to contribute to the development of therapies to treat familiar DCM, we have carefully evaluated potential therapeutic target sites in TTN gene. Importantly, symmetric exons are statistically highly overrepresented in TTN, making this clinically relevant gene an extraordinary promising target for therapeutic reframing approaches. Selecting key exons highly accessible to recently developed genome editing tools would facilitate the establishment of novel CRISPR-based therapeutics to explore novel treatments for DCM.

\section{Results}

\subsection{Universal protocol for the generation of transgene-free iPSC from a New World Monkey, Old World Monkeys, and human}

We aimed to establish a universal protocol for NHP-iPSC generation and culture for both Old World monkeys (OWM) and New World Monkeys (NWM). Generating iPSC from the different species under standardized conditions facilitates direct comparability amongst them as well as with human iPSC. Previously, we have shown the robust generation of human, baboon and rhesus macaque iPSC. The episomal vector system published by Okita et al. (2011) in combination with UPPS medium (Universal Primate Pluripotent $\underline{\text { Stem cell }}$ medium) enabled the generation of a relatively broad panel of OWM and human iPSC (Chapter 2, and 3). As these conditions were not tested for any NWM species, we selected the marmoset monkey as a biomedically relevant NWM species. 


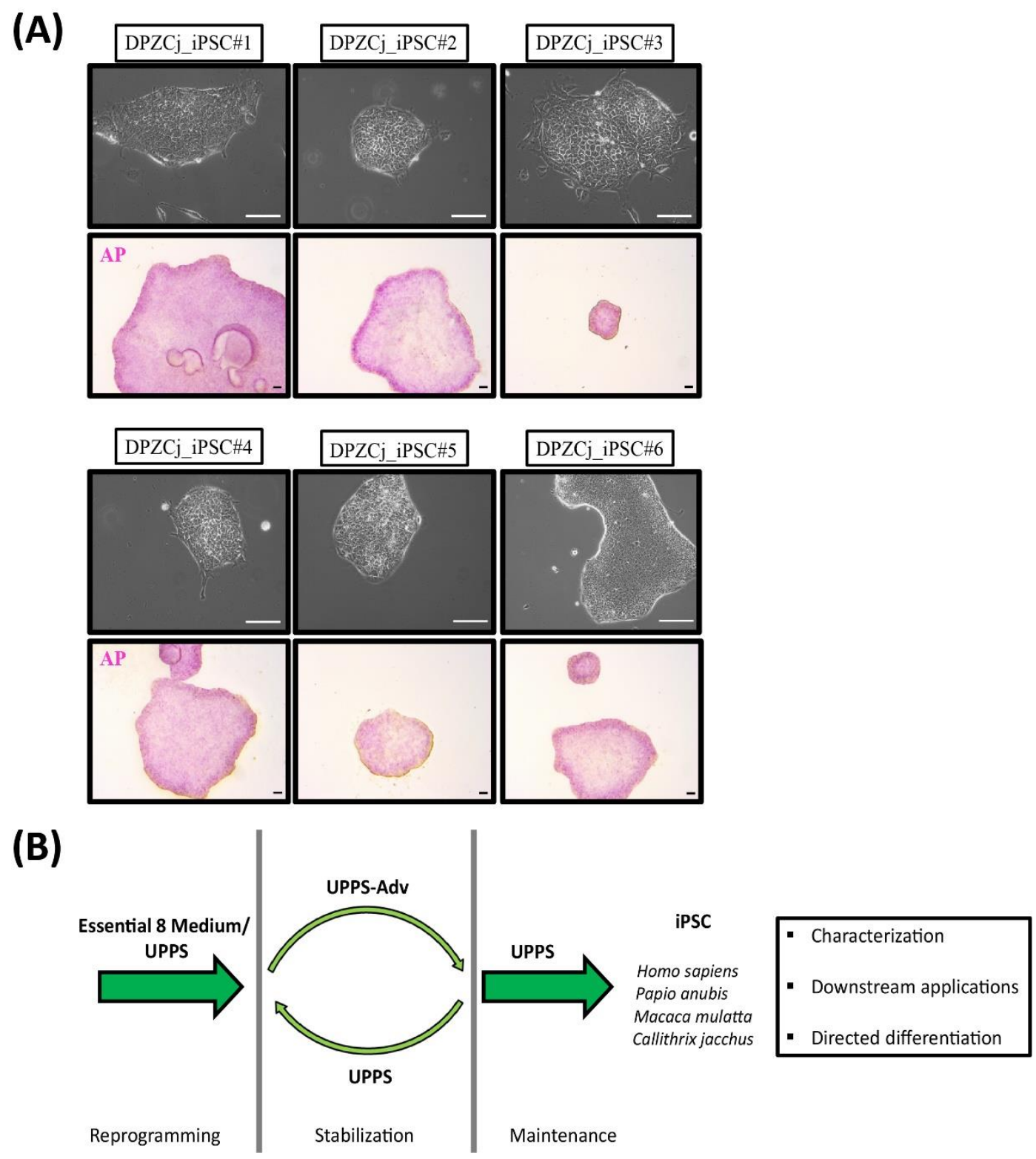

Figure 1: Marmoset induced pluripotent stem cells. (A) Six individual lines generated were generated (DPZCj_iPSC\#1-6). Upper row iPSC colony morphology, bright field pictures (scale bar $100 \mu \mathrm{m}$ ). Lower row, alkaline phosphatase staining (scale bar $100 \mu \mathrm{m}$ ). (B) Work-flow for the generation of primate iPSC. The protocol is divided into three different phases: 1-reprogramming, 2-stabilization of the generated lines, and 3-maintenance. UPPS (Universal Primate Pluripotent Stem cell medium), and UPPS-Adv (Universal Primate Pluripotent Stem cell medium, plus Activin A and LIF).

Fibroblasts extracted from skin biopsies from three different marmosets were cultured and expanded. We then applied our previously published protocol (Chapter 2) to the newly generated cells. Even though colonies with the typical morphology of pluripotent cells appeared in the primary plates, the expansion of these cells was not possible using UPPS medium. The cells differentiated, lost the typical cellular and colony morphology of PSC and stopped proliferation. Therefore we adjusted the protocol to find out suitable conditions to maintain also these marmoset cells. We tested different media (Essential 8 and MEF conditioned medium) and a broad range of small molecules, including dorsomorphin and recombinant Human Nodal Protein. None of the tested conditions was sufficient to keep marmoset iPSC in an undifferentiated state in feeder-free culture (data not shown). Only 
the addition of Activin A to the UPPS medium resulted in undifferentiated iPSC populations. The generated lines presented the typical high nucleus/cytoplasm ratio and grew in compact colonies with distinct borders (Fig. 1). We named this medium UPPS Advance (UPPS-Adv). However, even though cells remained undifferentiated, the proliferation rates were low. While macaque and baboon iPSC were split every 2-3 days (using splitting ratios 1:10/15), marmoset iPSC in UPPS-Adv were split every 5-6 days (using the same dilution for passaging of the cells). However, proliferation could be boosted by the addition of leukemia inhibitory factor (LIF). Nonetheless, marmoset iPSC elicit lower proliferation rates in comparison with rhesus and baboon in UPPS. After 6-10 passages in UPPS-Adv, the marmoset lines were again tested in UPPS and remained undifferentiated (Fig. 1, A). We then tested the capacity of rhesus, baboon, and human iPSC culture in UPPS-Adv . Human and OWM iPSC could also be maintained undifferentiated under the new conditions. All lines tested ( 2 human, 2 macaques, and one baboon line) were cultured for at least 3 passages and maintained in a morphologically undifferentiated state in UPPS-Adv (Suppl. Fig. 1, shown for one representative line of each species).

With the data presented here, we provide a universal protocol that can be used for reprogramming of human, rhesus, baboon, and marmoset monkey fibroblasts. This protocol comprises the initial culture of the iPSC during the process of establishment (passage 1 to 10) in UPPS-Adv medium. During this phase, the iPSC stabilize and can be easily expanded. When a homogeneous population of iPSCs is reached it is then possible to switch to standard UPPS medium for the characterization and downstream applications (Fig. 1, B).

Using this protocol we generated 6 different marmoset iPSC lines (DPZCj_iPSC\#1-6) from three different animals. DPZCj_iPSC\#1 and 6 were generated from skin biopsies of two different newborn marmosets, and DPZCj_iPSC\#2-5 from fetal fibroblasts. As a preliminary evaluation of the pluripotency of the generated iPSC lines, we performed alkaline phosphatase staining. All generated lines show alkaline phosphatase activity (Fig. $1, \mathrm{~A})$.

\subsection{Marmoset iPSC characterization}

Before characterization of the novel marmoset iPSC lines, we tested them for the presence of the reprogramming episomes via PCR. Only one line shows PCR signals (DPZCj_iPSC\#1). The absence of signals in the other lines confirms that DPZCj_iPSC\#26 are transgene-free (Suppl. Fig. 2).

In order to identify specific molecular signatures of pluripotency in the generated lines, we analyzed the expression of key pluripotency factors on the mRNA and protein level, respectively. RT-PCR analysis was performed for endogenous OCT4A, KLF4, and c-MYC markers. All lines show an upregulation in the expression of the selected pluripotencyassociated genes in comparison with the negative control (fibroblasts) (Suppl. Fig. 3). Furthermore, we confirm the expression of pluripotency markers on the protein level by immunostaining. All lines express OCT4A, LIN28, NANOG, SOX2, TRA-1-81 and TRA1-60 (Suppl. Fig. 4) (shown for DPZCj_iPSC\#1, 2, 3, and 5). 
(A)

(B)
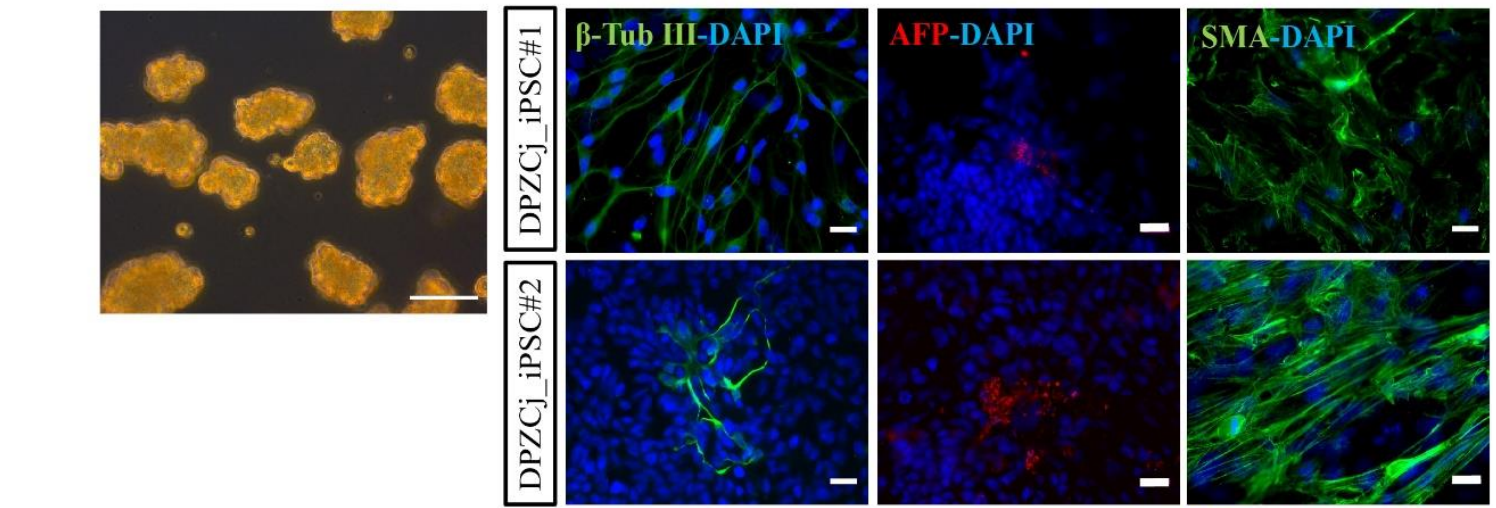

(C)
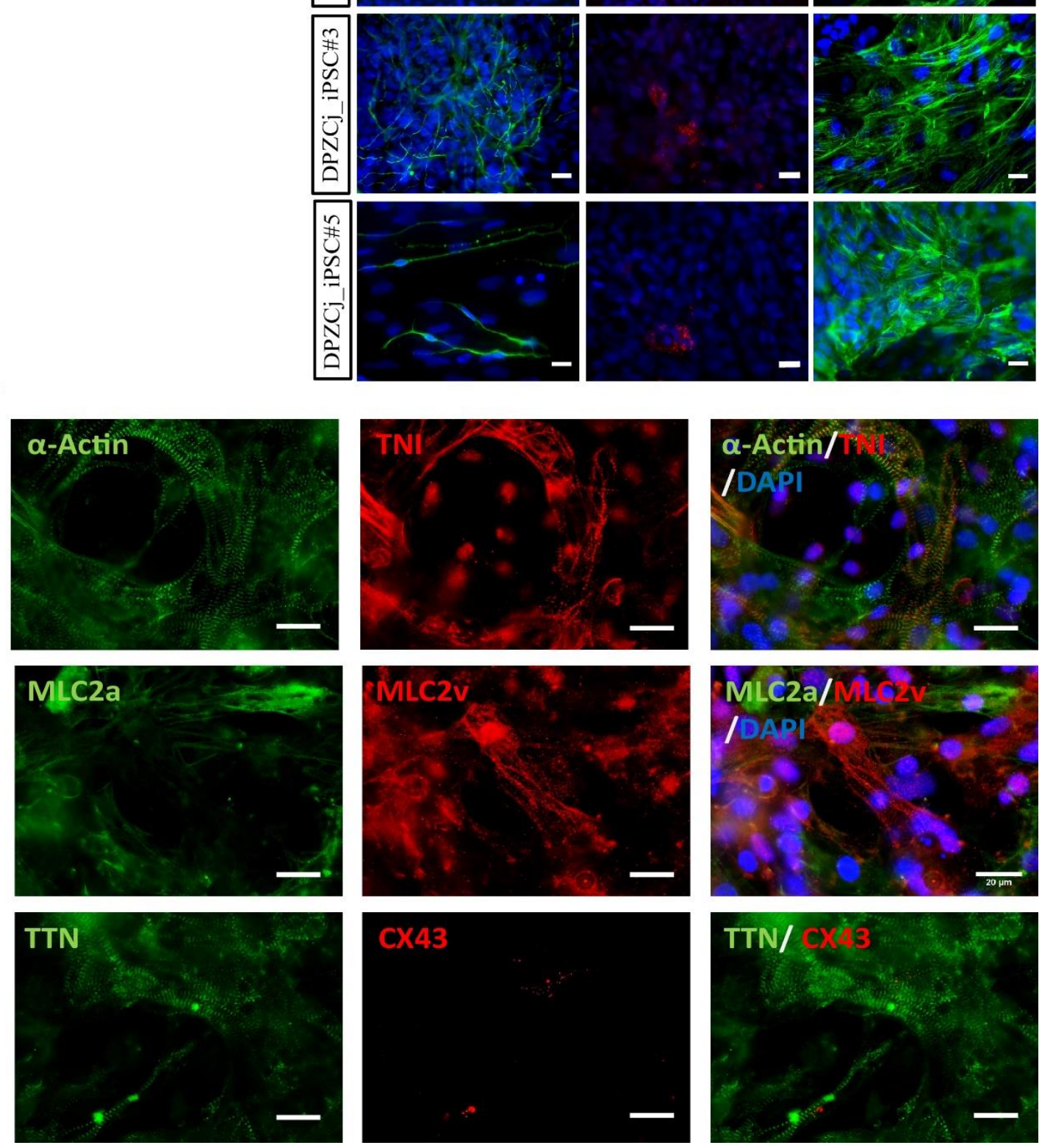

Figure 2: Differentiation of marmoset iPSCs. (A) (B) Pluripotency assessment by embryoid body formation of marmoset iPSC lines DPZCj_iPSC\#1, 2, 3 and 5. (A) Embryoid bodies exemplarily shown for DPZCj_iPSC\#3 (scale bar $100 \mu \mathrm{m}$ ). (B) Staining of the differentiated cells with representative markers of each embryonic germ layer: beta three Tubulin ( $\beta$-Tub III, ectoderm), Alpha-fetoprotein (AFP, endoderm), and smooth muscle actin (SMA, mesoderm) (scale bars $20 \mu \mathrm{m}$ ). (C) Directed differentiation of 
DPZC $j_{-}$iPSC\#1 into cardiomyocytes. Staining for muscle-specific markers alfa actin ( $\alpha$-Actin), troponin I (TNI), atrial and ventricular myosin light chain $2($ MLC2a/v), titin (TTN), and connexin 43 (Cx43) (scale bars $20 \mu \mathrm{m})$.

After characterization of the lines on the molecular level, we performed potency assessment by spontaneous differentiation. Embryoid body formation assay was used and differentiation evaluated by immunostaining. Smooth muscle cells positive to smooth muscle actin (SMA, mesoderm), hepatic-like cells stained by alpha-fetoprotein (AFP, endoderm), and neurons stained for beta III tubulin ( $\beta$-III-Tub, ectoderm) were found for all lines (Fig. 2, shown for DPZCj_1, 2, 3 and 5).

Additionally, directed differentiation into cardiomyocytes was tested for DPZCj_iPSC\#1, 2,3 , and 5 . We used our previously published protocol (Chapter 3 ) that has proven to be highly efficient for baboon, macaque, and human. DPZCj_iPSC\#1, 2, 3 and 5 developed into beating cells when exposed to the cardiomyocyte differentiation conditions. In order to confirm the identity of the generated iPSC-derived cardiomyocytes (iPSC-CM), we performed stainings for cardiomyocyte markers. The marmoset iPSC-CM expressed alfa actin ( $\alpha$-Actin), troponin I (TNI), atrial and ventricular myosin light chain 2 (MLC2a/v), titin (TTN), and connexin 43 (Cx43) (Fig. 2, C).

\subsection{Single-cell cloning of primate iPSC}

In recent years, many efforts have been made to generate clonal human and mouse iPSC lines. However, even though new protocols show increased efficiencies, the process is yet challenging. Given that little is known on NHP-iPSC clonal line establishment, we tested different protocols for the derivation of clonal NHP-iPSC lines.

We previously described a work-flow for the efficient generation of clonal lines from macaque iPSC. This protocol is based on the dissociation of cells using accutase solution, and cell sorting using UPPS medium supplemented with pro-survival factor (PSF) (Chapter 3). In order to evaluate the translatability of the protocol, we have tested it with human, baboon, and marmoset iPSC lines.

We evaluated the efficiency by counting the wells showing clonal expansion after FACS sorting. The complete work-flow was performed in parallel with the different lines from the four primate species consider in this study, human, macaque, baboon, and marmoset. The protocol was initiated three passages after transfection with the $p T T-P B-p C A G-e C a s 9$ GFP-U6-gRNA-Neo vector in combination with the transposase containing vector $p$ BasedtTomato. Primate iPSC were sorted according to the expression of the GFP reporter gene into 96 well plates, sorting one or three cells per well. Using the mentioned work-flow it was possible to isolate lines for all three primate species. The efficiency ranged from 5,55 to $19,4 \%$ (1 cell per well) (Fig. 3, A). As expected, when three cells per well were sorted the efficiency increased, reaching $79 \%$ for the marmoset (Fig. 3, B). In parallel, cell differentiation was evaluated by analyzing the morphology of the resultant colonies. While human and rhesus macaque clonal lines were stable, baboon and marmoset clones were more unstable. Stabilization of these lines was achieved by an additional cultivation step in UPPS-Adv (Fig.3, C). All generated clonal lines constitutively express GFP, demonstrating the stability of the expression (Fig.3, C). 
(A)

(B)
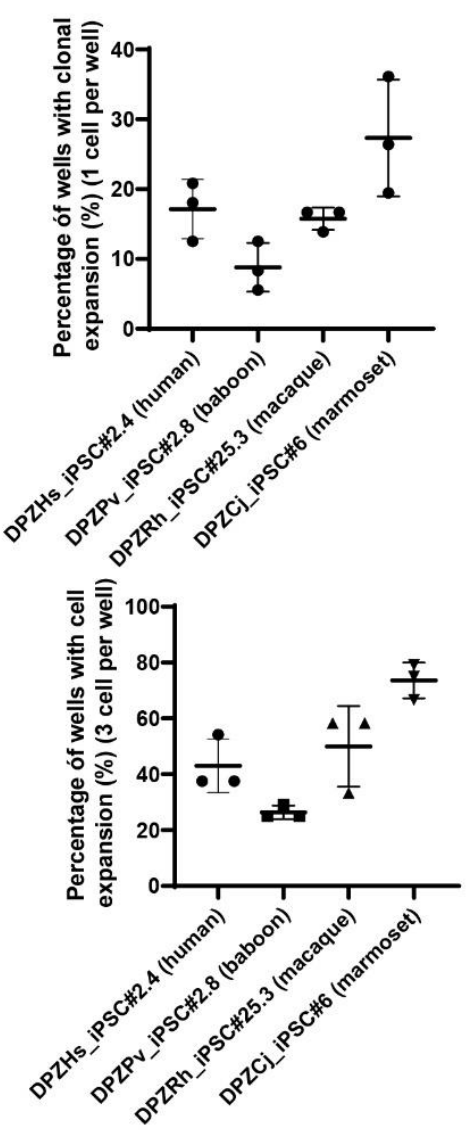

(C)
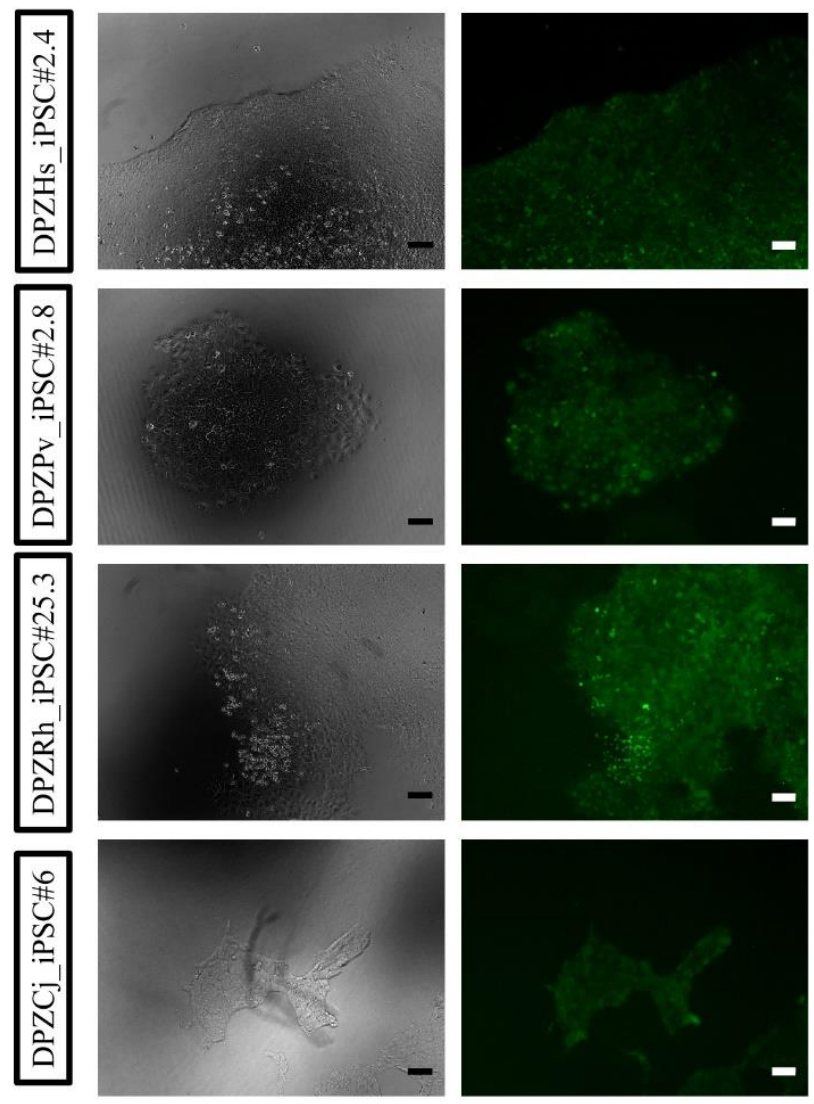

Figure 3: Clonal cell line derivation from primate iPSC. Human (DPZHs_iPSC\#2.4), macaque (DPZRh_iPSC\#25.3), baboon (DPZPv_iPSC\#2.8), and marmoset (DPZCj_iPSC\#6) (A)(B) Percentage of wells with cell expansion after single-cell dissociation and sorting into a 96 well plate. (A) Monoclonal line generation after sorting one cell per well. (B) Polyclonal line generation after sorting three cells per well. (C) Evidence of successful transgenesis. The iPSC express the GFP-Cas9 after sorting. Left panel, bright field pictures, right panel green fluorescence pictures of single clones growing in a well of a 96 well plate (scale bar $100 \mu \mathrm{m})$.

\subsection{Induction of INDEL mutations in primate iPSC}

CRISPR/Cas was utilized for the generation of single-cell clones with defined mutations using all four primate iPSC. We used a piggyBac-CRISPR/Cas system as backbone for single guide RNA ( $\operatorname{sgRNA}$ ) insertion to target defined locations in the primate genome. We selected two exons in the disease-relevant gene Titin (TTN). One location induced a frameshift mutation next to the N-terminus part of the protein (human, exon 38) and another proximal to the C-terminus (human, exon 280) (ENST00000589042.5). Both exons are highly conserved (PSI $>0,9)$ and clinically relevant since mutations in these positions have been found in DCM patients.

In silico analysis of the homologous TTN locations in the NHP species considered in this study was performed to guarantee the translatability of the editing approach. As final proof 
that the editing system can be used in all primate species, we performed $\mathrm{T} 7$ endonuclease I assay in marmoset fibroblasts and human HEK293 cells (sgRNA validation for the rhesus macaque was demonstrated in Chapter 3 ). Both guides designed show editing activity in all species (Fig.4, A, shown for marmoset).

Using the designed $\operatorname{sgRNAs}$ it was possible to generate monoclonal lines with INDEL mutations for human, rhesus, and marmoset. Targeting efficiency varies between lines and species. However, positive clones could be identified in all species.

(A)

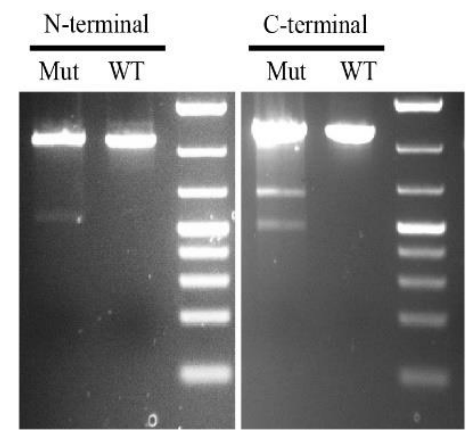

(B)

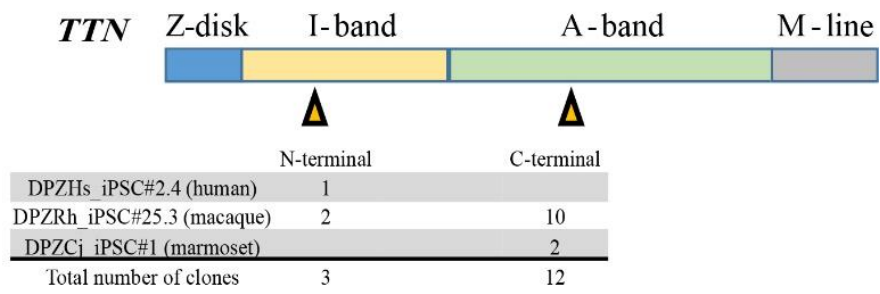

Figure 4: Genome editing in human and NHP iPSC. (A) sgRNA validation in marmoset fibroblast by T7 endonuclease I assay, for TTN N-terminal (human TTN, exon 38) and C-terminal (human TTN, exon 280) locations. Wildtype (WT) fibroblasts are shown in comparison with targeted fibroblasts (mutated, Mut). Amplicon size detailed in Suppl. Table 1 (B) Schematic representation of TTN gene and the two targeting sites. The number of clonal iPSC lines with INDEL mutations in the proposed sites. DPZHs_iPSC2.4 (human iPSC), DPZRh_iPSC\#25.3 (Rhesus macaque iPSC), and DPZCj_iPSC\#1 (marmoset iPSC).

We identified one human iPSC clone (DPZHs_iPSC\#2.4) with a deletion in exon 38 of TTN (total number of clones analyzed: 2). Additionally, two clonal lines from the marmoset monkey (DPZCJ_iPSC\#1) were generated with truncation mutations in exon 280 (total number of clones analyzed: 3) (Fig.4, B). Characterization of the mutations was performed by PCR amplification of the targeted genomic site and subsequent Sanger sequencing. Therefore, further analysis using refined methods will be required to identify the specific mutations in each of the alleles of the different clones.

\subsection{Fragment deletions in primate iPSCs using the combination of two CRISPR vectors}

The more precise way to induce patient mutations in cell or animal models is by homologydirected repair (HDR) compared to the introduction of INDELs. Using this method it is possible to predefine the genetic modification that will be induced in the target genome. In contrast, using non-homologous end joining (NHEJ) the repair after cutting the genome is random. Therefore, induction of a defined modification is more challenging. We have demonstrated that editing of NHP-iPSCs is possible using NHEJ reparation mechanism (Chapter 3 for macaque iPSC) (Fig. 4, human, marmoset and macaque iPSC). As an 
intermediate step between the already described approach and HDR, we test the potential of the described system to perform deletions. Combining two sgRNAs with Cas9, it is possible to target two genomic locations at the same time and delete DNA fragments.

In order to test this approach, we designed two sgRNAs to target the intronic sequences flanking human TTN exon 280 (ENST00000589042.5). The sgRNAs were inserted in the pTT-PB-pCAG-eCas9-GFP-U6-gRNA-Neo backbone, and the system was tested in marmoset fibroblasts (Fig. 5, A). We performed PCR to amplify the target location. For the wildtype, we expected an amplicon size of $1117 \mathrm{bp}$, whereas a size of $762 \mathrm{bp}$ was expected for the mutated allel. PCR showed a faint band of 762bp. The identity of the fragment was confirmed by sequencing which revealed that the combination of two sgRNAs was able to delete a fragment of 389 bp corresponding to exon 280 (Fig. 5, A, B, and B').

The experiment was repeated using human (DPZHs_iPSC\#2.4) and marmoset (DPZCJ_iPSC\#1) iPSC. In this case, due to time limitations, it was not possible to generate monoclonal lines. Nevertheless, it was possible to show PCR bands corresponding to the deletion of exon 280 in all marmoset and human polyclonal iPSC populations (Fig. 5 C, and D). Analysis of the mutated populations was performed by PCR (Fig. 5 C, and D) and confirmed by subsequent sequencing of the respective amplicon (data not shown).

(A)

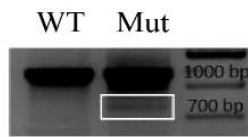

(B)

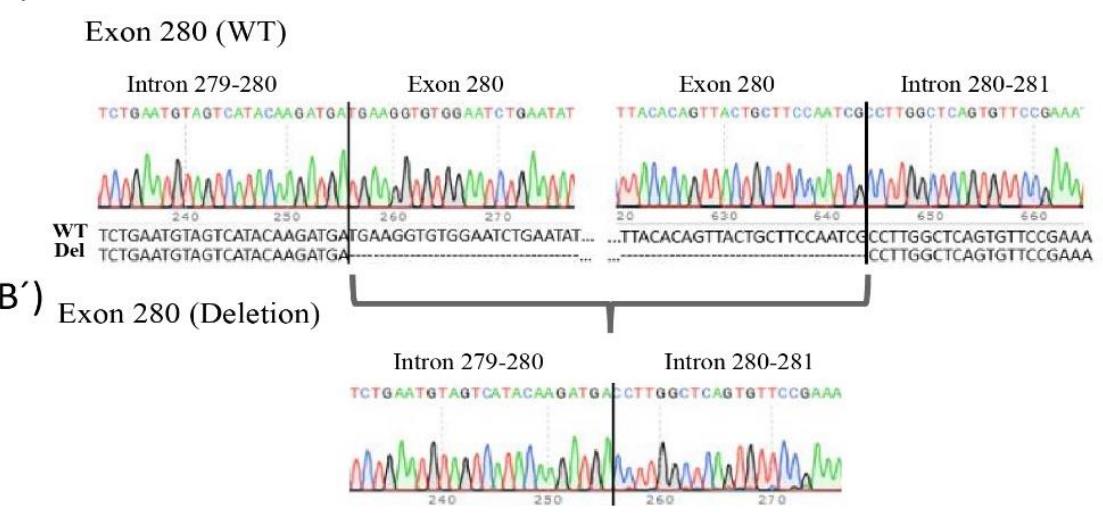

(C)

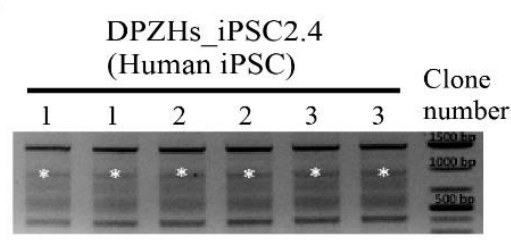

(D)

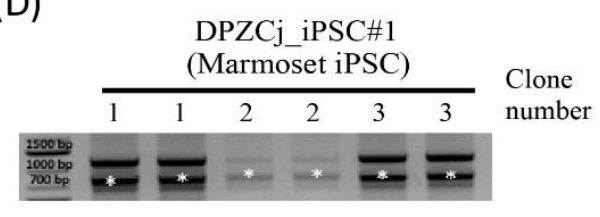

Figure 5: Deletion of human TTN exon 280 by a two sgRNA-CRISPR systems. (A) Validation of the system by transfection of marmoset fibroblasts. PCR designed to amplify the deletion site. Expected size of the amplicon in the wildtype (WT) is $1117 \mathrm{bp}$. In contrast in the targeted fibroblasts (mutated, Mut), both fragments were present, the non-edited fragment (1117 bp) and the fragment with the deletion (728bp) after 
removal of 389bp corresponding to the homologous human TTN exon 280. (B) (B') Sequencing of the amplicon in the mutated cells (Deletion $\left(\mathbf{B}^{\prime}\right)$ ) shows specific removal selected sequence in comparison with the wildtype (WT (B)). (C) (D) Fragment deletion in (C) human (DPZHs_iPSC2.4), and (D) marmoset (DPZCj_iPSC\#1) iPSC. PCR analysis (in duplicates) of three (1-3) different polyclonal populations of cells of two primate species. Two fragments are present in each PCR, the upper one corresponding to the unedited/INDEL mutated locus, and the lower one indicating the deletion $(*)$.

\subsection{Ventricular fibroblast immortalization}

The combination of iPSC containing clinically relevant mutations with robust cardiomyocyte generation protocols will allow evaluation of phenotypic alterations in species-specific iPSC-CM. Nonetheless, we are aware that some cardiovascular disease phenotypes cannot be fully simulated in $2 \mathrm{D}$ cultures systems. Therefore, it will be necessary to adapt the cells to culture systems that allow the generation of organotypic responses. Many groups have generated platforms that allow these analyses. Most of them combine iPSC-CM with fibroblast to mimic the natural composition of the myocardium.

In order to support and enhance this transition, we have extracted ventricular fibroblasts from the marmoset monkey. The extracted fibroblasts were immortalized and labeled using two different piggyBac systems. Immortalization was performed by exogenous expression of the human telomerase (PTT-PB-hTERT-puro) ${ }^{125}$. Labeling was performed by expression of a red fluorescence reporter (PTT-PB-dtTomato-puro) (Fig. 1 A). Red fluorescence was selected for labeling since the mutated iPSC lines we generated expressed the Cas9-GFP fusion transcript. Therefore, after the combination of green cells (primate iPSC-CM containing patient genotype) and red cells (species-tissue-specific fibroblasts), it will be possible to track both cell types and separate them from each other based on their fluorescence for downstream analyses.

Immortalized and labeled fibroblasts were cultured in parallel with wild-type (WT) fibroblasts as control. Around passage $10 \mathrm{WT}$ cells lost their proliferation capacity and became senescent. In contrast, immortalized fibroblasts maintained stable proliferation independent of the passage number (Fig. 6 A, compare with B). Constitutive expression of the telomerase was evaluated by RT-PCR with primers that cover the telomerase transcript. The immortalized fibroblasts in passage 16 show a higher telomerase expression in comparison to the WT (Fig. 6 C). 


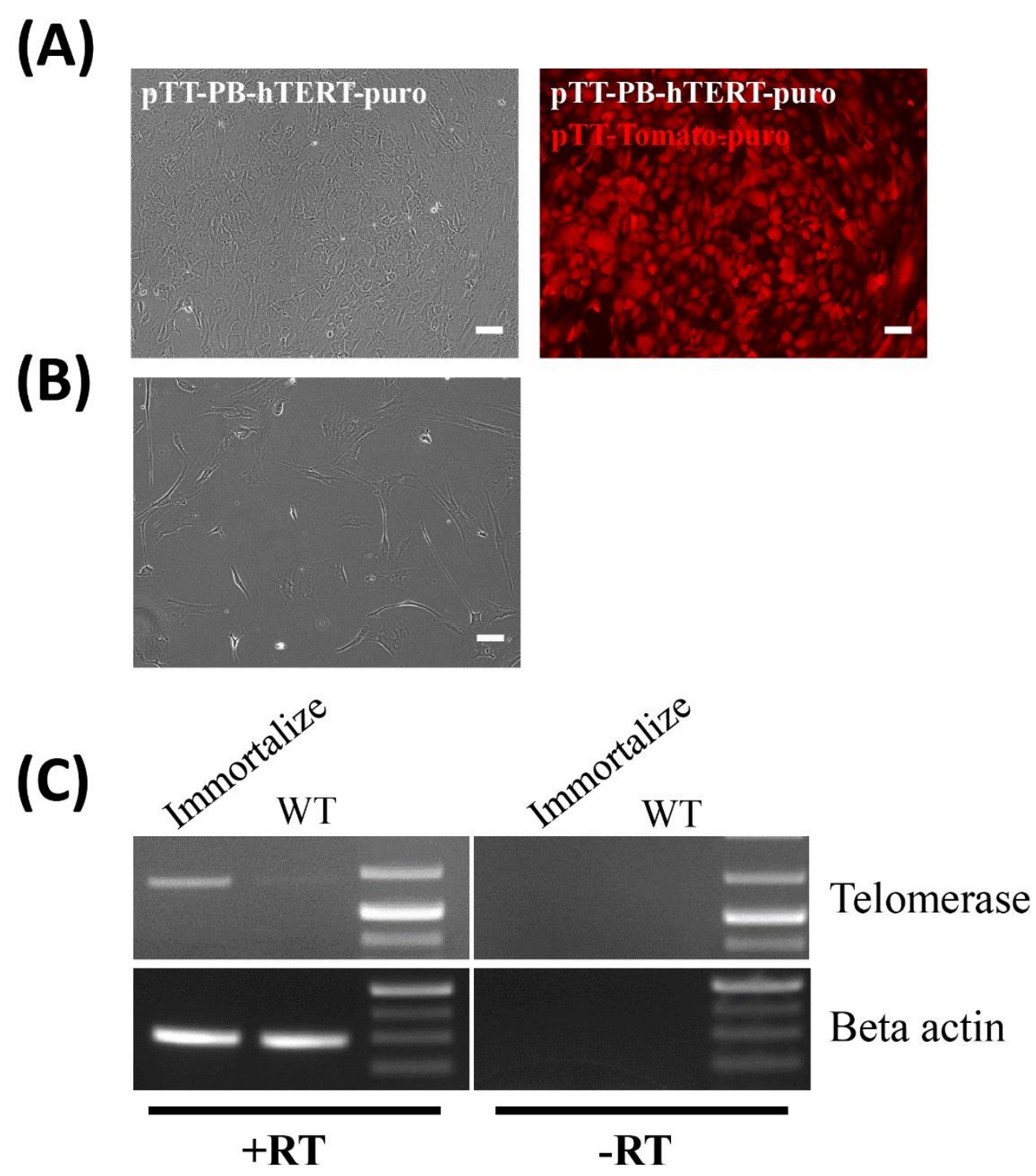

Figure 6: Marmoset ventricular fibroblasts immortalization and labeling. (A) Fibroblasts transfected with PTT-PB-hTERT-puro and PTT-PB-dtTomato-puro vectors at passage 16. Left picture bright field, right picture red fluorescence (scale bar $100 \mu \mathrm{m}$ ). (B) Bright field picture of wildtype ventricular fibroblasts in the same passage show signs of senescence (scale bar 100 $\mu \mathrm{m}$ ). (C) RT-PCR for telomerase (amplicon size 653 bp), comparing expression between wildtype (WT) and transfected cells (immortalized). Beta-actin used as housekeeping gene (amplicon size 285bp).

\subsection{Titin symmetric exon deletion}

Based on the intensive analysis of TTN across species we notice the very high percentage of symmetric exons in this gene (Fig. 7, A). This gene has higher number of exons with a nucleotide number multiple of three in comparison with other sarcomeric and cardiac relevant genes (Fig. 7, B) (Suppl. Table 3). Additionally, the number of symmetric exons does not strongly vary between the main isoforms. The TTN metatranscript contains $85 \%$ (311/363) of symmetric exons, and the different isoforms range between 67 and 83\% (Novex-3 and the long cardiac isoform N2BA respectively) (Fig. 7, A). 
Considering these results together with the feasibility to perform medium size deletions previously shown, we derived an empirical approach to threat DCM caused by mutations in $T T N$. We hypothesize that a complete deletion of symmetric exons containing mutations will not influence overall protein function, but restore the reading frame of TTN.

In order to demonstrate the feasibility of this therapeutic strategy, the first step was the identification of appropriate symmetric exons in TTN (Fig. 7, A) (Suppl. Table 4). However, in order to select candidate exons suitable for deletion, some additional considerations must be taken into account. Targeted exons should be highly conserved amongst different isoforms, i.e. they should have a high PSI (percentage of splice in) value. A PSI value of 1 indicates that an exon is constitutively present in all isoforms. A PSI value close to zero indicates that the respective exon is present only in very few transcripts and absent from most. Mutations located in TTN exons with low PSI usually lead to mild phenotypes. Finally, exon size also needs to be considered. We assume that selection of small or medium-sized exons (300 bp or smaller) will allow to perform the deletions with higher efficiency and prevent chromosomal alterations. In addition, is necessary to be sure that restricting the exon size we still consider most TTN exons (Suppl. Fig. 5). This will be particularly important regarding preclinical and also clinical in vivo testing of these therapies (Fig. 7).

In accordance with the criteria mentioned above, we have selected a set of candidate exons for the deletion from the TTN metatranscript (Suppl. Table 5). All selected exons are (1) symmetric, (2) highly conserved and have a PSI of $>0.9$, and (3) have 300 or less bp (Fig. 7, C) (Suppl. Fig. 5). A library of 94 candidate exons that fulfill all requirements was identified (Suppl. Table 5). We propose to apply an editing system composed of Cas9 and two gRNAs to target the intronic flanking sequences of selected exons of TTN. The whole system can be easily validated in the developed iPSC/CRISPR platform. Finally the most promising candidates for the potential therapeutic deletion of mutated TTN exons can be then tested in vivo models. 
(A)

(B)

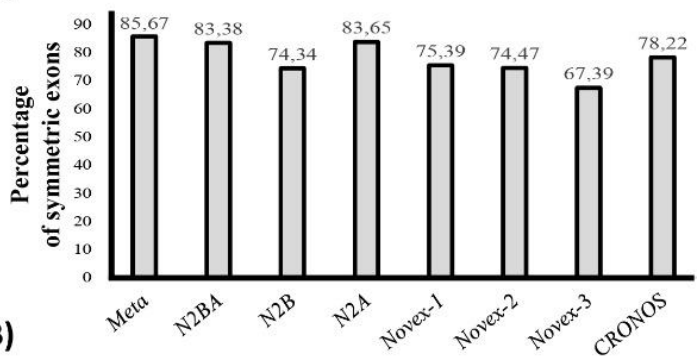

(C)

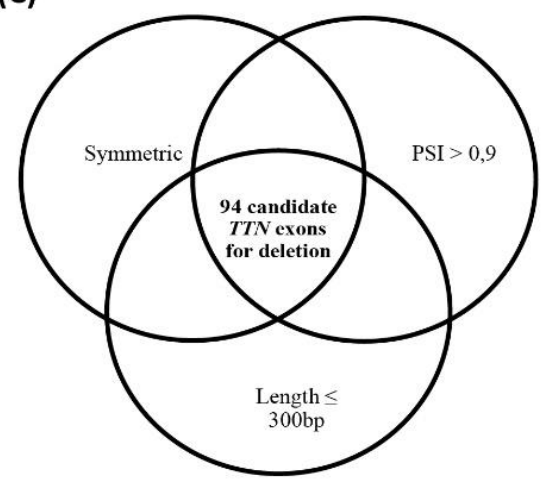

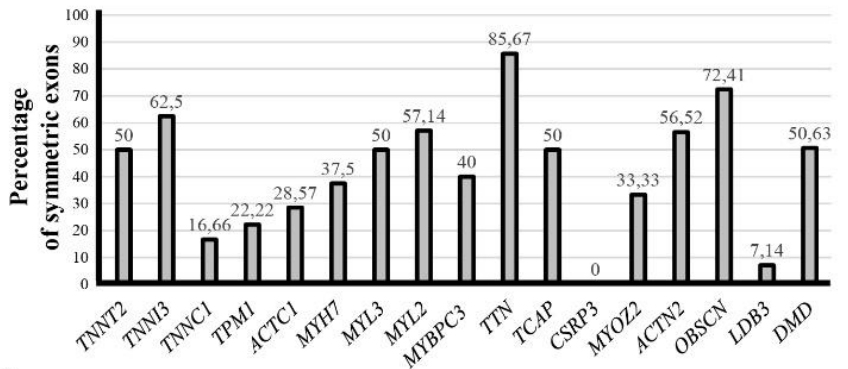

(E)

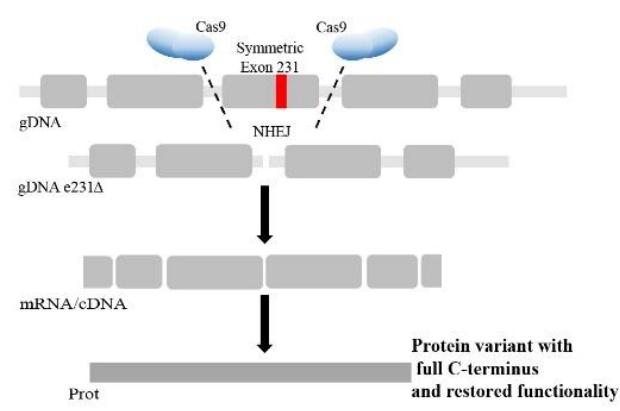

Figure 7: (A) (B) Percentage of symmetric exons quantification. (A) Percentage of symmetric exons in the major Titin isoforms. Metatranscript (311 symmetric exons / 363 exons in total, 85,67\%), N2BA (261/313, $83,38 \%)$, N2B (142/191, 74,34\%), N2A (261/312, 83,65\%), Novex-1 (144/191, 75,39\%), Novex-2 (143/192, 74,47\%), Novex-3 (31/46, 67,39\%) and Cronos (97/124, 78,22\%). (B) Percentage of symmetric exons in different cardiac genes. TNNT2 (8/16, 50\%), TNNI3 (5/8, 62,5\%), TNNCl (1/6, 16,67\%), TPM1 (2/9, 22,21\%), ACTC1(2/7, 28,57\%), MYH7 (15/40, 37,5\%), MYL3 (3/6, 50\%), MYL2 (4/7, 57,14\%), MYBPC3 (14/35, 40\%), TTN (311/363, 85,67\%), TCAP (1/2, 50\%), CSRP3 (0/7, 0\%), MYOZ2 (2/6, 33,33\%), ACTN2 (13/23, 56,52\%), OBSCN (84/116, 72,41\%) and $L D B 3(1 / 14,7,14 \%)$ and $D M D(40 / 79,50,63 \%)$. (C) Schematic representation of the parameters considered for target exon identification. Selected exons are symmetric, highly conserved (PSI>0.9) and have maximally 300bp. (D) (E) Overview of the exon deletion therapy approach. Symmetric exons with mutations are deleted with a system combining Cas9 plus two gRNAs, targeting sites up- and downstream of the candidate exon. TTN expression results in a shorter but functional protein. (D) Schematic representation of a frameshift mutation in exon 231 of TTN that leads to a truncated and partially non-sense version of the protein. The red color indicates a non-sense amino acid sequence different from TTN that originates from the frameshift mutation present in exon 231. The frameshift causes at the same time the occurrence of a stop codon on the transcript level, which in turn results in a truncated protein variant. (E) Schematic representation of the deletion of a symmetric exon to remove the frameshift mutation located in exon 231. 


\section{Material and methods}

\subsection{Animals and animal housing; ethics statement}

The German Primate Center (DPZ) is an independent research institute authorized by the veterinary governmental authorities (Reference number: 122910.3311900, PK Landkreis Göttingen). Marmoset monkey skin samples were made available during the pathological examination, from animals from an unrelated project. DPZCj_iPSC\#2-5 were generated from fetal fibroblasts extracted from a marmoset developing embryo. DPZCj_iPSC\#1 and 6 were generated from skin biopsies of two different new-born marmosets. Human, rhesus macaque and baboon iPSC derivation as described previously (Chapter 2).

\subsection{Isolation of marmoset primary fibroblasts}

Marmoset fibroblasts were extracted according to ${ }^{29}$. In brief, skin biopsies were washed with PBS (1\% (v/v) Penicillin/Streptomycin (Gibco), 0,25 $\mu \mathrm{g} / \mathrm{mL}$ Amphotericin B (Sigma)) and minced with a scalpel. Following, the tissue was digested for 3 hours with Collagenase Type IV solution $\left(10 \mathrm{mg} / \mathrm{mL}\right.$ in DMEM) $($ Gibco $)\left(37^{\circ} \mathrm{C}\right.$, rotating at $\left.80 \mathrm{rpm}\right)$. Next, undigested dermal tissue was removed, and the cell suspension centrifuged ( $5 \mathrm{~min}$, $300 \mathrm{x}$ g, RT). The resultant pellet was resuspended in Rh15 medium [DMEM (Gibco), 15\% (v/v) Fetal Bovine Serum (Gibco), 1\% (v/v) Penicillin/Streptomycin (Gibco), 0,1\% (v/v) Amphotericin B (Sigma), 1\% (v/v) MEM Non-Essential Amino Acids Solution (Gibco), $2 \mathrm{mM}$ GlutaMAX (Gibco), $10 \mathrm{ng} / \mathrm{ml} \mathrm{bFGF}$ ] and cultured in a cell culture plate coated with gelatine $\left(0,1 \%\right.$ gelatine; Sigma). Primary cultures were maintained at $37^{\circ} \mathrm{C}$ and $5 \% \mathrm{CO} 2$. Accutase (Gibco) was used for passaging.

\subsection{Reprogramming NHP fibroblasts}

Reprogramming was performed according to Chapter 2. In brief marmoset fibroblasts were nucleofected (4D-Nucleofector, P2 Primary Cell Solution (Lonza)) with the three plasmids pCXLE-hOCT3/4-shp53-F (\#27077), pCXLE-hSK (\#27078) and pCXLE-hUL (\#27080) published by Okita et al. (2011) ${ }^{30} .6 \times 10^{5}$ cells were transfected with $6 \mu \mathrm{g}$ ( $2 \mu \mathrm{g}$ each) of pDNA. Fibroblasts were kept in Rh15 medium supplemented with $0.5 \mathrm{mM}$ sodium butyrate (Sigma-Aldrich) for 7 days, changing the medium every day. At day 7 the transfected cells were transferred to Geltrex $(0.16 \mathrm{mg} / \mathrm{ml}$; Thermo Fisher) coated plates and cultured in Essential 8 medium (Thermo Fisher) supplemented with sodium butyrate until day 11. From day 12 on only Essential 8 medium was applied. Approximately 30 days after transfection the first distinct colonies appeared. 
Primary colonies were picked and maintained in UPPS-Adv medium [StemMACS ${ }^{\text {TM }}$ iPSBrew XF, human, $1 \mu \mathrm{M}$ IWR-1, (Sigma-Aldrich), $0.5 \mu \mathrm{M}$ Chir99021 (Merck), 4 ng/ml activin A (Miltenyi Biotec), $10 \mathrm{ng} / \mathrm{mL}$, Recombinant Human LIF (PeproTech)] for the first 6-10 passages. Cells were maintained in these conditions until a homogeneous population of cells was reached. Cells were passaged using versene solution. (Thermo Fisher Scientific). Around passage 15 cells stabilized and were subsequently cultured in standard UPPS (Chapter 2).

To evaluate the possibility of maintaining human and OWM iPSC in the newly developed culture conditions, cell lines were differentially cultured in both UPPS and UPPS-adv. These conditions were maintained for minimum 3 passages, carefully evaluating morphology and proliferation rates.

\subsection{In vitro differentiation}

Embryoid body formation was performed for the marmoset iPSC lines. iPSC colonies were digested with collagenase type IV (200 U/ml; Worthington) for $10 \mathrm{~min}$ at $37^{\circ} \mathrm{C}$. After digestion, colonies were resuspended with the help of a cell scraper, trying to conserve the integrity of colonies. Cell clusters were placed in suspension in a non-adherent petri dish and cultured in differentiation medium [79 ml Iscove's Modified Dulbecco's Medium (IMDM, Thermo Fisher), $20 \mathrm{ml}$ FBS, $1 \mathrm{ml} \mathrm{100x}$ non-essential amino acids, $450 \mu \mathrm{M} 1$ thioglycerol (Sigma-Aldrich)]. On day 8 cell clusters were transferred to Geltrex coated plates with coverslips. On transferring, Embryoid Bodies settled down and attached, allowing cells to expand and differentiate. Differentiated cells were maintained for 17 days (days 25 in total) before fixation and staining. Antibodies and dilutions used are described in Suppl. Table 2.

\subsection{Cardiac differentiation of marmoset iPSC}

The protocol for cardiac differentiation described in Chapter 2 was applied. The protocol consists of two different phases, mesodermal differentiation, and cardiac specification differentiations. First primate iPSCs were exposed to mesodermal induction medium [RPMI 1640, B27 without insulin, $1 \mathrm{mM}$ sodium pyruvate (Thermo Fisher), $200 \mu \mathrm{M} \mathrm{L}$ ascorbic acid 2-phosphate, $1 \mu \mathrm{M}$ Chir99021, 9 ng/ml Activin A (Miltenyi Biotec, premiumgrade) and $5 \mathrm{ng} / \mathrm{ml} \mathrm{BMP4} \mathrm{(Miltenyi} \mathrm{Biotec,} \mathrm{premium} \mathrm{grade].} \mathrm{The} \mathrm{medium} \mathrm{was} \mathrm{changed} \mathrm{on}$ day 1. On day three cardiac specification is induced by application of cardiomyocyte differentiation medium [RPMI 1640, B27 supplement without insulin, $1 \mathrm{mM}$ sodium pyruvate, $200 \mu \mathrm{M}$ L-ascorbic acid 2-phosphate, $5 \mu \mathrm{M}$ IWR-1]. The medium was further changed on day 5. From day 7 on, cardiomyocytes are maintained in cardiomyocyte maintenance medium [RPMI 1640, B27 with insulin, $200 \mu \mathrm{M}$ L-ascorbic acid 2phosphate]. 
3.6 Immunostaining

Immunostaining was performed according to the protocol described in ${ }^{31}$. In brief, cells were washed with DPBS (Thermo Fisher) and fixed using 4\% (w/v) paraformaldehyde (Merck) for 20 min (room temperature). Blocking was performed with 1\% bovine serum albumin (Thermo Fisher), supplemented for staining of intracellular epitopes with $0.1 \%$ Triton X-100 (Sigma-Aldrich). Primary antibody incubation was performed overnight at $4^{\circ} \mathrm{C}$ (primary antibodies used detailed in, Suppl. Table 2). After incubation with the primary antibody, cells were washed three times with PBS and incubated for one hour with the secondary antibody (secondary antibodies used detailed in, Suppl. Table 2). Subsequently DAPI ( $1 \mu \mathrm{g} / \mathrm{ml}$ DAPI in H2O (Sigma-Aldrich)) staining was performed for $10 \mathrm{~min}$ at room temperature. Stained cells were mounted using Fluoromount-G (eBioscience) and analyzed by fluorescence microscopy (Zeiss).

\subsection{DNA and RNA isolation and polymerase chain reaction}

Genomic DNA was extracted using DNeasy Blood \& Tissue Kit (Qiagen) following the manufacturer's instructions. Presence of the episomes was analyzed by PCR using specific primers for the amplification of conserved sequences between the three used episomes (Table 1). Each PCR (final volume $50 \mu \mathrm{l}$ ) was performed with 50-100 ng DNA using Taq DNA Polymerase with Standard Taq Buffer (New England Biolabs) following manufacturers instructions.

RNA was extracted from frozen pellets using NucleoSpin RNA Plus isolation kit (Macherey-Nagel), and treated with RNase-free DNase (Qiagen) to eliminate contaminant genomic DNA. Reverse transcription of $1 \mu \mathrm{g}$ RNA was performed using Omniscript RT kit (Qiagen). cDNA was amplified using marmoset specific primers for the different pluripotency factors, all detailed in Suppl. Table 1.

\subsection{T7 endonuclease I assay}

Validation of the sgRNA was performed in marmoset fibroblasts, transfecting the cells with $6 \mu \mathrm{g}$ pDNA, using 4D nucleofector, and P2 nucleofection solution (Lonza). After at least 3 days, fibroblasts were snap-frozen and the genomic DNA extracted as described in (3.7). Primers for specific amplification of the two locations in the TTN gene (Suppl. Table 1) were designed to generate an amplicon of approximately $1 \mathrm{~kb}$ with the potential mutation in the middle. PCR products were purified using NucleoSpin DNA RNA and protein purification kit (Macherey Nagel). T7 endonuclease assay was performed using T7 Endonuclease I (NEB) according to manufacturer recommendations.

\subsection{Clonal expansion analysis}

One iPSC line for each primate species considered in the study was transfected with $p$ BasedtTomato and pTT-PB-pCAG-eCas9-GFP-U6-gRNA-Neo according to the protocol 
described in Chapter 3. Every transfection was performed in triplicates, and three passages after transfection the cells were sorted using SH800S Cell Sorter (Sony Biotechnology), according to Cas9-GFP expression. Each experimental group was sorted in a 96 well plate, sorting 1 (72 wells) or 3 (24 wells) cells per well. Clonal expansion was evaluated by counting of wells with undifferentiated, proliferating cells(Mean $\pm \mathrm{SD}$, using GraphPad PRISM).

\subsection{Induction of truncation and deletion mutations in primate iPSC}

Construction of the vector $p T T-P B-p C A G-e C a s 9-G F P-U 6-g R N A-N e o$ from $p C A G-e C a s 9$ GFP-U6-gRNA (Addgene \#79145) was described in Chapter 3. Two guide RNAs were cloned into the CRISPR/Cas9 vector (Suppl. Table 1). Potential mutated clones were FACS-sorted in order to obtain clonal sublines, expanded, and pelleted for gDNA extraction and analysis (Macherey-Nagel ${ }^{\mathrm{TM}}$ NucleoSpin $^{\mathrm{TM}}$ Gel and PCR Clean-up Kit). Primers for amplification of the potential mutated sites were described in (3.8) and are detailed in Suppl. Table 1. PCR products of the different clones were purified (NucleoSpin DNA RNA and protein purification kit, Macherey Nagel) and sequenced (Sanger) (LGC Genomics). Sequence analysis was performed using Serial Cloner, Poly Peak Parser ${ }^{126}$, and MEGA software.

\subsection{NHP ventricular fibroblast immortalization}

Two adult marmoset hearts were dissected. Two biopsies from the ventricle were extracted and washed in PBS [1\% (v/v) Penicillin/Streptomycin (Gibco), 0,25 $\mu \mathrm{g} / \mathrm{mL}$ Amphotericin $\mathrm{B}$ (Sigma)]. Clean heart tissue was minced with a scalpel and digested for 2 hours using Collagenase Type IV solution $\left(10 \mathrm{mg} / \mathrm{mL}\right.$ in DMEM) $($ Gibco $)\left(37^{\circ} \mathrm{C}\right.$, rotating at $\left.80 \mathrm{rpm}\right)$. Cells were centrifuged ( $5 \mathrm{~min}, 300 \mathrm{x} \mathrm{g}, \mathrm{RT}$ ) and resuspended in culture medium [DMEM (Gibco), 15\% (v/v) Fetal Bovine Serum (Gibco), $1 \%$ (v/v) Penicillin/Streptomycin (Gibco), 0,1\% (v/v) Amphotericin B (Sigma), 1\% (v/v) MEM Non-Essential Amino Acids Solution (Gibco), $2 \mathrm{mM}$ GlutaMAX (Gibco), $10 \mathrm{ng} / \mathrm{ml} \mathrm{bFGF]} \mathrm{and} \mathrm{transferred} \mathrm{to} 10 \mathrm{~cm}$ gelatine coated plates $(0,1 \%$ gelatine; Sigma). Primary cultures were split with Accutase (Gibco) $\left(37^{\circ} \mathrm{C}\right.$ and $\left.5 \% \mathrm{CO}_{2}\right)$.

Ventricular fibroblasts were immortalized and fluorescent-labeled by co-transfection of piggyBac vectors containing human telomerase gene (PTT-PB-hTERT-puro $)^{28}, \quad$ and Tomato fluorescent reporter ( $P T T-P B-d t$ Tomato-puro). All transposon transfections were done in combination with $p$ Base-dtTomato. Transfected cells were selected with puromycin $(1 \mu \mathrm{g} / \mathrm{ml})$ (Thermo Fisher Scientific) until a population of pure tomato positive cells was reached (approx. 3 passages). Non-transfected ventricular fibroblasts were cultured in parallel in order to compare proliferation and morphology of the cells. 


\section{Discussion}

Given the high incidence of CVD in the population, animal models for investigation of the initiation and progression of the disease are required. The generation of NHP models will facilitate corresponding research in a systematic and controlled experimental setup ${ }^{83}$. The relevance of NHP for translational studies is reflected by the increased number of disease and biological studies using these models in the last years ${ }^{34-39}$. Especially Old wold monkeys (OWM) have been used to study the influence of genetic variation on CVD risk ${ }^{34} 35$, caloric restriction and CVD ${ }^{36}$, Chagas disease ${ }^{37} 38$ or stem cell-based therapeutics for the cardiovascular system among others 83940 . While OWM are well established in biomedical research, NWM are gaining only now more interest this field ${ }^{41} 42$.

For the generation of genetically modified NHP containing disease-related mutations, it is necessary to be able to induce specific genetic modifications using genome editing systems 267 . Additionally, it is necessary to validate these tools prior to their application in vivo. For this purpose, NHP-iPSC represent an excellent alternative due to their shared similarities with PSC of the early monkey embryo and their differentiation capacity ${ }^{943}$.

We have established an in vitro preselection platform for the validation of genetic modification tools in NHP. In the present study, we have adapted our previously reported reprogramming and iPSC culture protocol (Chapter 2) to the particularities of the marmoset. Marmoset monkeys are animal models traditionally used in biomedical research during the last decades ${ }^{10}$. They have a primate-specific genetic constitution and additional advantages like shorter generation times, easy handling and lower housing cost in comparison with other NHP models, e.g., the macaque or the baboon ${ }^{9} 41$. We have generated 6 different marmoset iPSC lines under feeder-free conditions. Furthermore, for reprogramming we have used an episomal system resulting in five transgene-free iPSC out of six lines tested ${ }^{30}$.

The generated iPSC were characterized according to the standards established for human iPSC ${ }^{44}$. We have demonstrated the reactivation of key endogenous pluripotency markers on the mRNA and protein levels, respectively. Furthermore, we evaluated the potency of the different lines by embryoid body differentiation, demonstrating that these cells can differentiate into representative cell types from all three embryonic germ layers. We believe that additional in vivo differentiation assays are required considering that the generated lines could be used for transplantation projects. Therefore we injected the generated lines for teratoma assay. Due to time limitations, we do not know whether the cells form teratomas or not.

Considering the versatility of the cells, they can be used to study different human pathologies. However, as we aim to use them in the context of CVD, we tested directed 


\section{Chapter 4: An iPSC-based preselection platform for disease-inducing genetic modifications in non-human primates}

differentiation towards cardiomyocytes. The four lines tested generated cardiomyocytes following the previously reported work-flow (Chapter 2) adapted from the publication of Tiburcy and colleagues ${ }^{46}$.

Once the marmoset iPSC were fully characterized, we tested if the modified protocol based on the combination of UPPS / UPPS-Adv medium and Geltrex surface coating of the culture dish could be used for other species of primates. Rhesus macaque, human and baboon iPSC could be cultured in the new conditions showing no signs of differentiation. Therefore, we have established a work-flow that allows maintenance of iPSC from human, two OWM and one NWM species under identical conditions. This allows evaluation of genome editing tools considering a broad range of NHP models and additionally conduct developmental ${ }^{474}$ and evolutionary studies 49 .

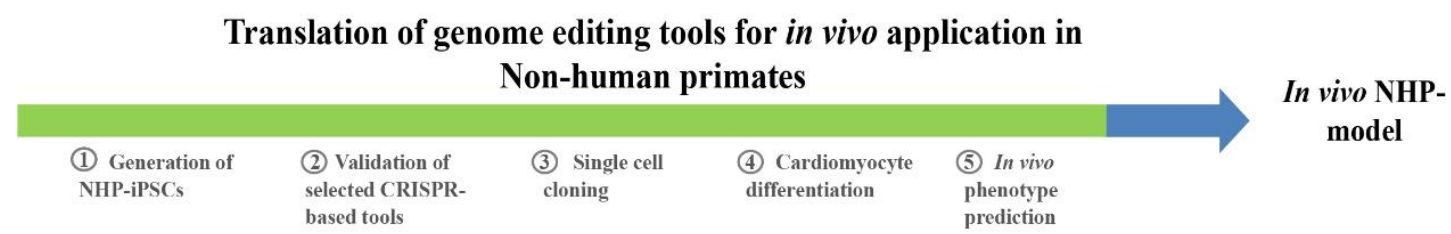

Figure 7: Schematic representation of the proposed work-flow for validation of genome editing tools in NHPiPSC.

Due to their cellular particularities, the generation of genetically modified PSC is challenging ${ }^{50}$. For the generation of iPSC lines with defined mutations, it is crucial to be able to derive clonal cell lines. We have tested a single cell sorting protocol that allows the generation of monoclonal primate iPSC lines. Furthermore, using the versatility of the piggyBac system, we were able to induce clinically relevant mutations in human, rhesus, and marmoset iPSC genomes.

Familiar dilated cardiomyopathy (DCM) is an inherited disease with a high incidence in the general population ${ }^{5152} 53$. This pathology is caused in $25 \%$ of the cases by truncating mutations in the sarcomeric protein TTN ${ }^{52}$. Generating animal models that share the genotype of the patients will allow the development of novel therapeutics. We targeted primate iPSC to induce frameshift mutations in locations affected in patients of DCM with severe phenotypes ${ }^{51}$. Even though the mutations have not been fully characterized, we have demonstrated for the first time the possibility of inducing mutations with high-efficiency in different preclinically relevant primate species.

Two different approaches for the genetic modification were used, both based on NHEJ repair of the DNA after cutting. First, we tested the system to induce point mutations in a specific location ${ }^{7}$. Once the protocol was established, we used the system for DNA 
fragment deletion. We used two different CRISPR/Cas vectors, to target adjacent locations in the primate genome. We are aware that for precise induction of clinically relevant mutations in animal models, the preferred path is HDR ${ }^{9}$. However, we believe that our tools developed in this study will help to bring these kinds of gene therapy closer to in vivo testing in NHP models 9 .

Additionally, derived from the study of the $T T N$ structure, we have empirically developed a potential therapeutic approach to DCM caused by a subset of TTN mutations. The high number of symmetric exons in TTN makes it an excellent candidate for a target gene for myoediting strategies. We have generated a library of potential target exons (94) to test exon deletion for reframing tvTTN variants in DCM patients. The 363 exons were categorized according to symmetry, size, and PSI. The list of potential therapeutic sites needs to be carefully validated using the described platform.

Finally, for in vitro research on DCM, it will be necessary to use culture systems with increased complexity that allow the identification of organotropic responses ${ }^{465}$. In order to facilitate this transition we have immortalized ventricular fibroblasts from the marmoset. Usually, patient-derived iPSC-CM are combined with fibroblasts in order to improve maturation and mimic the natural composition of the myocardium ${ }^{46}$. Although we made the first steps to establish NHP iPSC-CM / fibroblast co-culture systems, tissue-specific fibroblast extraction for the remaining species is still required. Since fibroblasts acquire tissue-specific traits. e.g. they express tissue-specific collagens; we consider it instrumental for organo-typic in vitro testing to use also tissue-specific fibroblasts. This may be particularly true for the mechanically stressed heart tissue.

In conclusion, we have generated a NHP iPSC-based in vitro pre-selection platform that allows the validation of genetic modifications prior to their in vivo application in NHP. The progress made not only represents an important contribution to the generation of preclinical disease models, but also to the 3Rs (reduce, refine, replace) in animal experimentation. 


\section{References (Chapter 4)}

1. Singhal, M. \& Kansara, N. Transgenic animals: Production and application. IJPSR 1, 12-22 (2010).

2. Dow, L. E. Modeling disease in vivo with CRISPR/Cas9 Lukas. Trends Mol Med 21, 609-621 (2016).

3. Burgio, G. Redefining mouse transgenesis with CRISPR / Cas9 genome editing technology. Genome Biol. 19, 1-3 (2018).

4. Zhao, J., Lai, L., WeizhiJi \& Zhou, Q. Genome editing in large animals: current status and future prospects. Natl Sci Rev 6, 402-420 (2019).

5. Sato, K. \& Sasaki, E. Genetic engineering in nonhuman primates for human disease modeling. J. Hum. Genet. 63, 125-131 (2017).

6. Qiu, P. et al. BMAL1 knockout macaque monkeys display reduced sleep and psychiatric disorders. Natl Sci Rev 6, 87-100 (2019).

7. Volobueva, A. S. \& Orekhov, A. N. An update on the tools for creating transgenic animal models of human diseases - focus on atherosclerosis. Braz J Med Biol Res 52, 511 (2019).

8. Cox, L. A. et al. Nonhuman Primates and Translational Research - Cardiovascular Disease. ILAR J 58, 235-250 (2017).

9. Yoshimatsu, S. et al. Robust and efficient knock-in in embryonic stem cells and early- stage embryos of the common marmoset using the CRISPR-Cas9 system. Sci. Rep. 9, 1-12 (2019).

10. Sasaki, E. et al. Generation of transgenic non-human primates with germline transmission. Nature 459, 523-527 (2009). 
11. Halevy, T. \& Urbach, A. Comparing ESC and iPSC - Based Models for Human. J. Clin. Med. 3, 1146-1162 (2014).

12. Bilic, J. \& Izpisua-Belmonte, J. C. Concise Review: Induced Pluripotent Stem Cells Versus Embryonic Stem Cells: Close Enough or Yet Too Far Apart? Stem Cells 30, 33-41 (2012).

13. Marei, H. E., Althani, A., Lashen, S., Cenciarelli, C. \& Hasan, A. Genetically unmatched human iPSC and ESC exhibit equivalent gene expression and neuronal differentiation potential. Sci. Rep. 7, 1-13 (2017).

14. Gigli, M. et al. A Review of the Giant Protein Titin in Clinical Molecular Diagnostics of Cardiomyopathies. Front. Cardiovasc. Med. 3, 1-9 (2016).

15. Robert G Weintraub, Christopher Semsarian, P. M. Dilated cardiomyopathy. Lancet 390, 400-414 (2017).

16. Braunwald, E. Cardiomyopathies: An overview. Circ. Res. 121, 711-721 (2017).

17. Gramlich, M. et al. Antisense-mediated exon skipping : a therapeutic strategy for titin-based dilated cardiomyopathy. EMBO Mol. Med. 7, 562-576 (2015).

18. Herman, D. S. et al. Truncations of Titin Causing Dilated Cardiomyopathy. $N$ Engl J Med. 366, 619-628 (2012).

19. Hershberger, R. E., Hedges, D. J. \& Morales, A. Dilated cardiomyopathy: The complexity of a diverse genetic architecture. Nat. Rev. Cardiol. 10, 531-547 (2013).

20. Zou, J. et al. An internal promoter underlies the difference in disease severity between $\mathrm{N}$ - and C-terminal truncation mutations of Titin in zebrafish. Elife 4, 1-22 (2015).

21. Hinson, J. T. et al. Titin Mutations in iPS cells Define Sarcomere Insufficiency as a Cause of Dilated Cardiomyopathy. Science. 349, 982-986 (2015).

22. Lattanzi, A. et al. Correction of the Exon 2 Duplication in DMD Myoblasts by a Single CRISPR / Cas9 System. Mol. Ther. Nucleic Acid 7, 11-19 (2017). 


\section{Chapter 4: An iPSC-based preselection platform for disease-inducing genetic}

modifications in non-human primates

23. Lalonde, S. et al. Frameshift indels introduced by genome editing can lead to inframe exon skipping. PLoS One 12, 1-13 (2017).

24. Tabebordbar, M. et al. In vivo gene editing in dystrophic mouse muscle and muscle stem cells. Science. 351, 407-411 (2016).

25. Nelson, C. E. et al. In vivo genome editing improves muscle function in a mouse model of Duchenne muscular dystrophy. Science. 351, 403-407 (2016).

26. Zhu, P. et al. CRISPR/Cas9-Mediated Genome Editing Corrects Dystrophin Mutation in Skeletal Muscle Stem Cells in a Mouse Model of Muscle Dystrophy. Mol. Ther. - Nucleic Acids 7, 31-41 (2017).

27. Gu, C. et al. A single CRISPR-Cas9 deletion strategy that targets the majority of DMD patients restores dystrophin function in hiPSC-derived muscle cells. Cell Stem Cell 18, 533-540 (2016).

28. Petkov, S. et al. Immortalization of common marmoset monkey fibroblasts by piggyBac transposition of hTERT. PLoS One 13, 1-13 (2018).

29. Debowski, K. et al. Non-viral generation of marmoset monkey iPS cells by a sixfactor-in-one-vector approach. PLoS One 10, 1-21 (2015).

30. Okita, K. et al. A more efficient method to generate integration-free human iPS cells. Nat. Methods 8, 409-412 (2011).

31. Rodriguez-polo, I. et al. Baboon induced pluripotent stem cell generation by piggyBac transposition of reprogramming factors. Primate Biol., 6, 75-86 (2019).

32. Hill, J. T. et al. Poly Peak Parser: Method and software for identification of unknown indels using Sanger Sequencing of PCR products. Dev Dyn. 243, 1632-1636 (2015).

33. Camacho, P., Fan, H., Liu, Z. \& He, J. Large Mammalian Animal Models of Heart Disease. J. Cardiovasc. Dev. Dis. 30, 1-11 (2016). 
34. Vinson, A., Mitchell, A. D., Toffey, D., Silver, J. \& Raboin, M. J. Sex-Specific Heritability of Spontaneous Lipid Levels in an Extended Pedigree of Indian-Origin Rhesus Macaques ( Macaca mulatta ). PLoS One 8, 1-11 (2013).

35. Cox, L. A. et al. Identification of Promoter Variants in Baboon Endothelial Lipase That Regulate High-Density Lipoprotein Cholesterol Levels. Circulation 116, 1185-1196 (2007).

36. Colman, R. J. et al. Caloric restriction reduces age-related and all-cause mortality in rhesus monkeys. Nat. Commun. 5, 1-5 (2014).

37. Vitelli-avelar, D. M. et al. Cynomolgus macaques naturally infected with Trypanosoma cruzi -I exhibit an overall mixed pro-inflammatory / modulated cytokine signature characteristic of human Chagas disease. PLoS ONE Neglected Trop. Dis. 2, 1-18 (2017).

38. Zabalgoitia, M. et al. Electrocardiographic Findings in Naturally Acquired Chagasic Heart Disease in Nonhuman Primates. J. Electrocardiol. 36, 155-60 (2003).

39. Shiba, Y. et al. Allogeneic transplantation of iPS cell-derived cardiomyocytes regenerates primate hearts. Nature 538, 388-391 (2016).

40. Chong, J. J. H. \& Murry, C. E. Cardiac Regeneration Using Pluripotent Stem Cells - Progression to Large Animal Models. Stem Cell Res. 13, 654-665 (2014).

41. Kishi, N., Sasaki, E. \& Okano, H. Common marmoset as a new model animal for neuroscience research and genome editing technology. Dev. Growth Differ 56, 53-62 (2014).

42. Robert Marini et al. The Common Marmoset in Captivity and Biomedical Research. (Academic Press, 2018).

43. Tabel, V. A., Atsma, D. E., Mummery, C. L. \& Davis, R. P. Human pluripotent stem cell models of cardiac disease : from mechanisms to therapies. Dis. Model. Mech. 10, 1039-1059 (2017). 
44. Yang, G. et al. Standards for Deriving Nonhuman Primate-Induced Pluripotent Stem Cells , Neural Stem Cells and Dopaminergic Lineage. Int. J. Mol. Sci. 19, 1-15 (2018).

45. Sullivan, S. et al. Quality control guidelines for clinical-grade human induced pluripotent stem cell lines. Regen. Med. 13, 859-866 (2018).

46. Tiburcy, M. et al. Defined Engineered Human Myocardium with Advanced Maturation for Applications in Heart Failure Modelling and Repair. Circulation 32, 178182 (2017).

47. Zhu, Z. \& Huangfu, D. Human pluripotent stem cells : an emerging model in developmental biology. Development 717, 705-717 (2013).

48. Shi, Y., Kirwan, P., Smith, J., Robinson, H. P. C. \& Livesey, F. J. Human cerebral cortex development from pluripotent stem cells to functional excitatory synapses. Nat. Neurosci. 15, 477-486 (2012).

49. Wunderlich, S. et al. Primate iPS cells as tools for evolutionary analyses. Stem Cell Res. 12, 622-629 (2014).

50. Chen, Y. \& Pruett-miller, S. M. Improving single-cell cloning work flow for gene editing in human pluripotent stem cells. Stem Cell Res. 31, 186-192 (2018).

51. Mcnally, E. M. \& Mestroni, L. Dilated Cardiomyopathy: Genetic Determinants and Mechanisms. Circ. Res. 121, 731-748 (2017).

52. Verdonschot, J. A. J., Hazebroek, M. R., Ware, J. S., Prasad, S. K. \& Heymans, S. R. B. Role of Targeted Therapy in Dilated Cardiomyopathy: The Challenging Road Toward a Personalized Approach. J. Am. Heart Assoc. 8, 1-18 (2015).

53. Li, Y., Lang, P. \& Linke, W. A. Titin stiffness modifies the force- generating region of muscle sarcomeres. Sci. Rep. 6, 1-9 (2016).

54. Nugraha, B., Buono, M. F., von Boehmer, L., Hoerstrup, S. P. \& Emmert, M. Y. Human Cardiac Organoids for Disease Modeling. Clin Pharmacol Ther 105, 79-85 (2019). 


\section{Supplementary data (Chapter 4)}

\begin{tabular}{|c|c|c|c|}
\hline Oligo applications & Name & Sequences $5^{\prime}-3^{\prime}$ & Amplicon (bp) \\
\hline \multirow{10}{*}{ RT-PCR primers } & G0957_hTERT_fwd & CTGGACGATATCCACAGGGC & \multirow{2}{*}{653} \\
\hline & G0958_hTERT_rev & AAGTTCACCACGCAGCCATA & \\
\hline & G0022_OCT4_fwd & GATCGGATCCTTGGGGCGCCTTCCTTC & \multirow{2}{*}{510} \\
\hline & G0035_OCT4_rev & CAGGGTGATCCTCTTCTGCTTC & \\
\hline & G0091_KLF4_fwd & GGAAGACGATCTTGGCCCCG & \multirow{2}{*}{323} \\
\hline & G0021_KLF4_rev & GTACTCTAGACAGTGTGGGTCATATCCACTG & \\
\hline & G0079_c-MYC_fw & ATAAGAATGCGGCCGCACTGGATTTTTTTCGGGCAGTGG & \multirow{2}{*}{456} \\
\hline & G00142_c-MYC_rev & CCTGGATGATGATGTTTTTGATG & \\
\hline & G2204_beta actin_fw & GGTAGTTTCGTGGATGCCACA & \multirow{2}{*}{285} \\
\hline & G2205_beta actin_rev & GACCTGACTGACTACCTCATG & \\
\hline \multirow{2}{*}{ gRNAs point } & G1806_TTN_N-terminus_fw & CACCgGCTGACTACACCTTTGTGGC & \\
\hline & G1807_TTN_N-terminus_rev & AAACGCCACAAAGGTGTAGTCAGCc & \\
\hline \multirow{2}{*}{ mutation } & G1822_TTN_C-terminus_fw & CACCgGTTACTGCTTCCAATCGCCT & \\
\hline & G1823_TTN_C-terminus_rev & AAACAGGCGATTGGAAGCAGTAACc & \\
\hline \multirow{2}{*}{ Analysis point } & G2616_N-term_fw & GCATGGTGGCACAGAGTTGT & \multirow{2}{*}{1861} \\
\hline & G2617_N-term_rev & TAGACAGCTGCTAGGGACAC & \\
\hline \multirow{2}{*}{ mutation primers } & G2654_C-term_fw & GCGACTCTGCCCAACTACAT & \multirow{2}{*}{828} \\
\hline & G2655_C-term_rev & GCAGTGTTGGTGACTTCCTC & \\
\hline \multirow{2}{*}{ gRNAs exon } & G2367_280delups_2_fw & CACCGGTAGTCATACAAGATGATGA & \\
\hline & G2368_280delups_2_rev & AAACTCATCATCTTGTATGACTACC & \\
\hline \multirow{2}{*}{280 deletion } & G2369_280deldown_2_fw & CACCGGTTACTGCTTCCAATCGCCT & \\
\hline & G2370_280deldown_2_rev & AAACAGGCGATTGGAAGCAGTAACC & \\
\hline \multirow{2}{*}{ Analysis } & G20_Human_fw & CAACTTATTTTGGGAGGGATG & \multirow{2}{*}{$1461 / 977^{*}$} \\
\hline & G21_Human_rev & AACTTATTTTGGGAGGGATG & \\
\hline \multirow{2}{*}{ deletion primers } & G22_marmoset_fw & CACCGTGAGAGGCTCACATACA & \multirow{2}{*}{$1170 / 762 *$} \\
\hline & G23_marmoset_rev & AAACGTATCAAACACTATTTACTAAGGAG & \\
\hline \multirow{2}{*}{ Episomal } & G2000_Episomal_EBNA_fw & $\begin{array}{l}\text { GCTATTGCTTCCCGTATGGC } \\
\end{array}$ & \multirow{2}{*}{555} \\
\hline & G2001_Episomal_EBNA_rev & CAAAGGGAGATCCGACTCGT & \\
\hline \multirow{2}{*}{ detection } & G2002_Episomal_EBV_fw & GGTTCACTACCCTCGTGGAAT & \multirow{2}{*}{592} \\
\hline & G2003_Episomal_EBV_rev & CGGGGCAGTGCATGTAAT & \\
\hline
\end{tabular}

Suppl. Table 1: Oligos used in this study. (*) Product size (bp) for the wildtype and the deleted fragments.

\begin{tabular}{|c|c|c|}
\hline Antibody & Supplier & Dilution \\
\hline OCT-4A & Cell Signaling, 2890 & $1: 1600$ \\
\hline LIN 28A & Cell Signaling, 3978S & $1: 100$ \\
\hline NANOG & Cell Signaling, 4903 & $1: 400$ \\
\hline SOX2 & Cell Signaling, 3728 & $1: 200$ \\
\hline TRA 1-60 & eBioscience, 14-8863 & $1: 100$ \\
\hline TRA 1-81 & eBioscience, 14-8883 & $1: 100$ \\
\hline
\end{tabular}




\begin{tabular}{|c|c|c|}
\hline$\beta$-Tubulin III & Sigma, T8660 & $1: 400$ \\
\hline AFP & Dako, A0008 & $1: 200$ \\
\hline Sox9 & Millipore, AB5535 & $1: 200$ \\
\hline SMA & Sigma, A2547 & $1: 1000$ \\
\hline$\alpha$-actinin & Sigma, A7811 & $1: 1000$ \\
\hline Cx43 & bcam, ab11370 & $1: 1000$ \\
\hline cTNI & Abcam, ab47003 & $1: 200$ \\
\hline cTNT & Thermo Fisher, MS295PABX & $1: 200$ \\
\hline Titin & Merck, MAB1553 & $1: 50$ \\
\hline Alexa 488 conjugated goat- $\alpha$-mousse IgM & Thermo Fisher Scientific, A21042 & $1: 1000$ \\
\hline Alexa 488 conjugated goat- $\alpha$-mousse IgG & Thermo Fisher Scientific, A10667 & $1: 1000$ \\
\hline Alexa 488 conjugated donkey- $\alpha$-rabbit IgG & Thermo Fisher Scientific, A21206 & $1: 1000$ \\
\hline Alexa 488 conjugated donkey- $\alpha$-mouse IgG & Thermo Fisher Scientific, A21202 & $1: 1000$ \\
\hline
\end{tabular}

Suppl. Table 2: Antibodies used in this study.

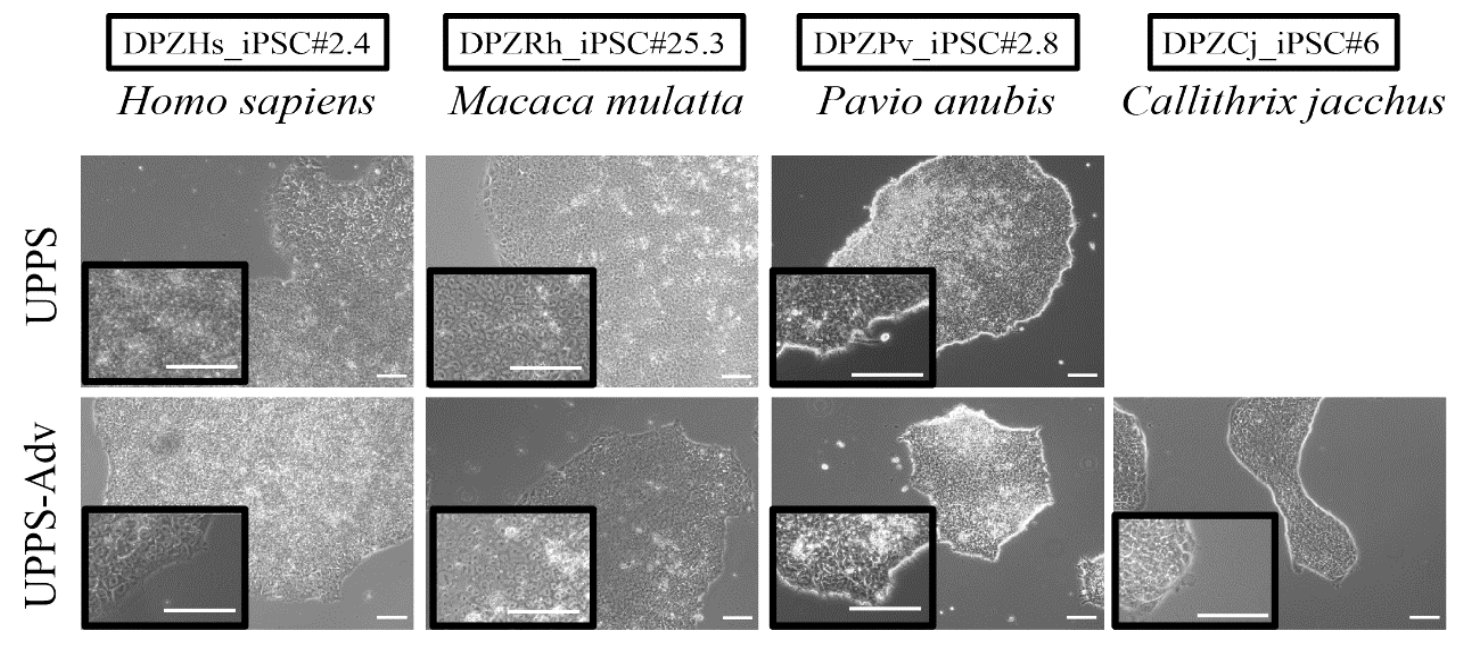

Suppl. Fig 1: Comparison of primate iPSC morphology after three passages in UPPS and UPPS-Adv. Comparison performed for the four primate species considered in this project. Human (DPZHs_iPSC\#2.4), macaque (DPZRh_iPSC\#25.3), baboon (DPZPv_iPSC\#2.8), and marmoset (DPZCj_iPSC\#6) (scale bar $100 \mu \mathrm{m})$. 


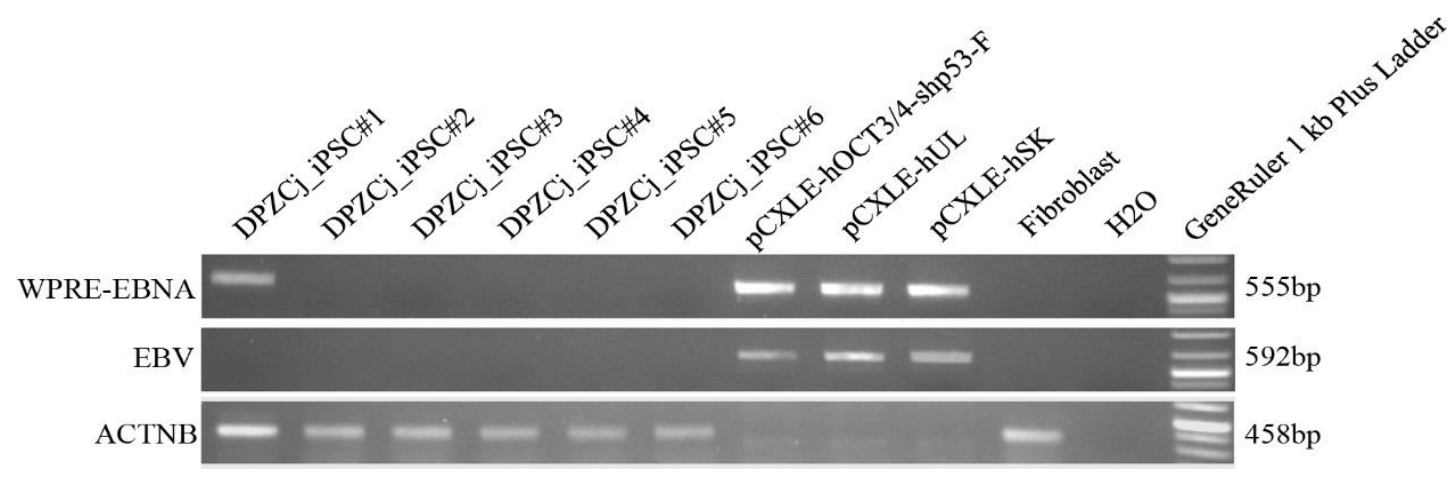

Suppl. Fig 2: Detection of the reprogramming episomal vectors by PCR. Two different primer pairs specific for the vectors were used (WPRE and EBV). Amplicon size in base pairs (bp) detailed on the right. Betaactin was used as control for the presence of DNA (ACTNB). Plasmid DNA from the three reprogramming constructs $p C X L E-h O C T 3 / 4, p C X L E-h U L$, and $p C X L E-h S K$ was used as positive control. Wildtype marmoset fibroblasts were used as negative control. Only DPZCj_iPSC\#1 shows a signal for the detection of the episomes, suggesting that the episomes are still present.

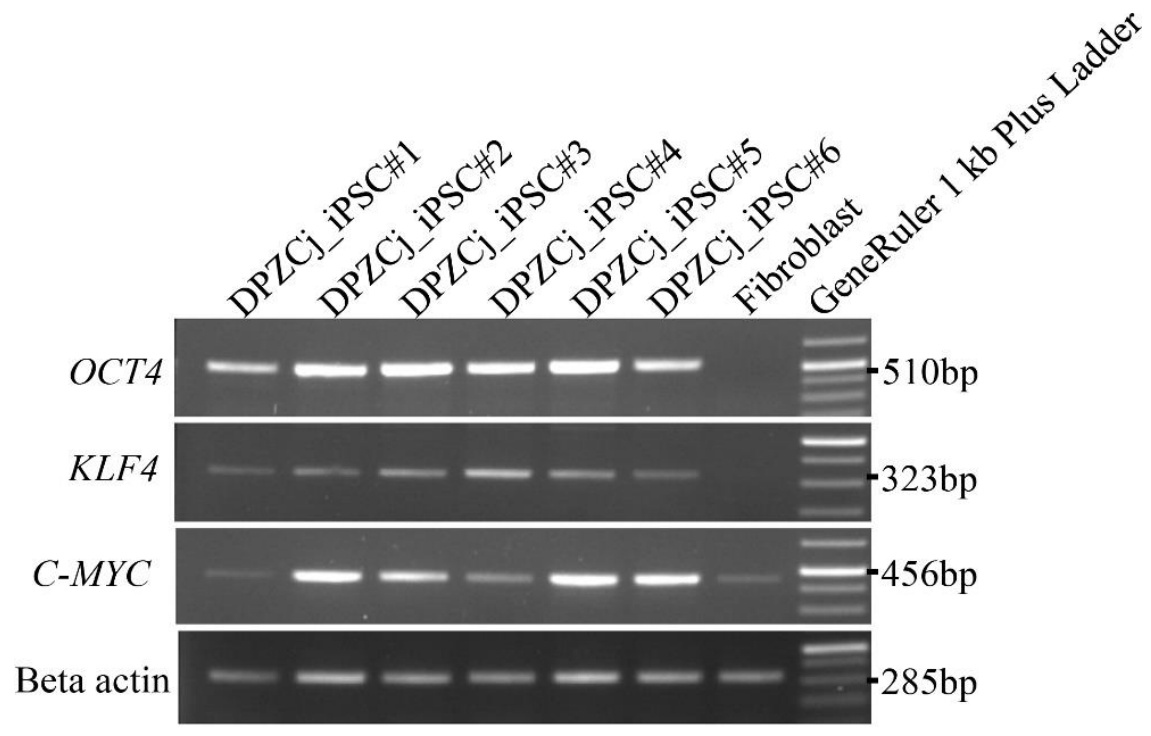

Suppl. Fig 3: RT-PCR for the marmoset lines DPZCj_iPSC\#1-6. Semi-quantitative analysis of the expression of the endogenous pluripotency genes OCT4, KLF4, $c-M Y C$. Beta-actin was used as internal control. Amplicon size detailed on the right (base pairs, bp). Marmoset fibroblasts were used as biological negative control. 


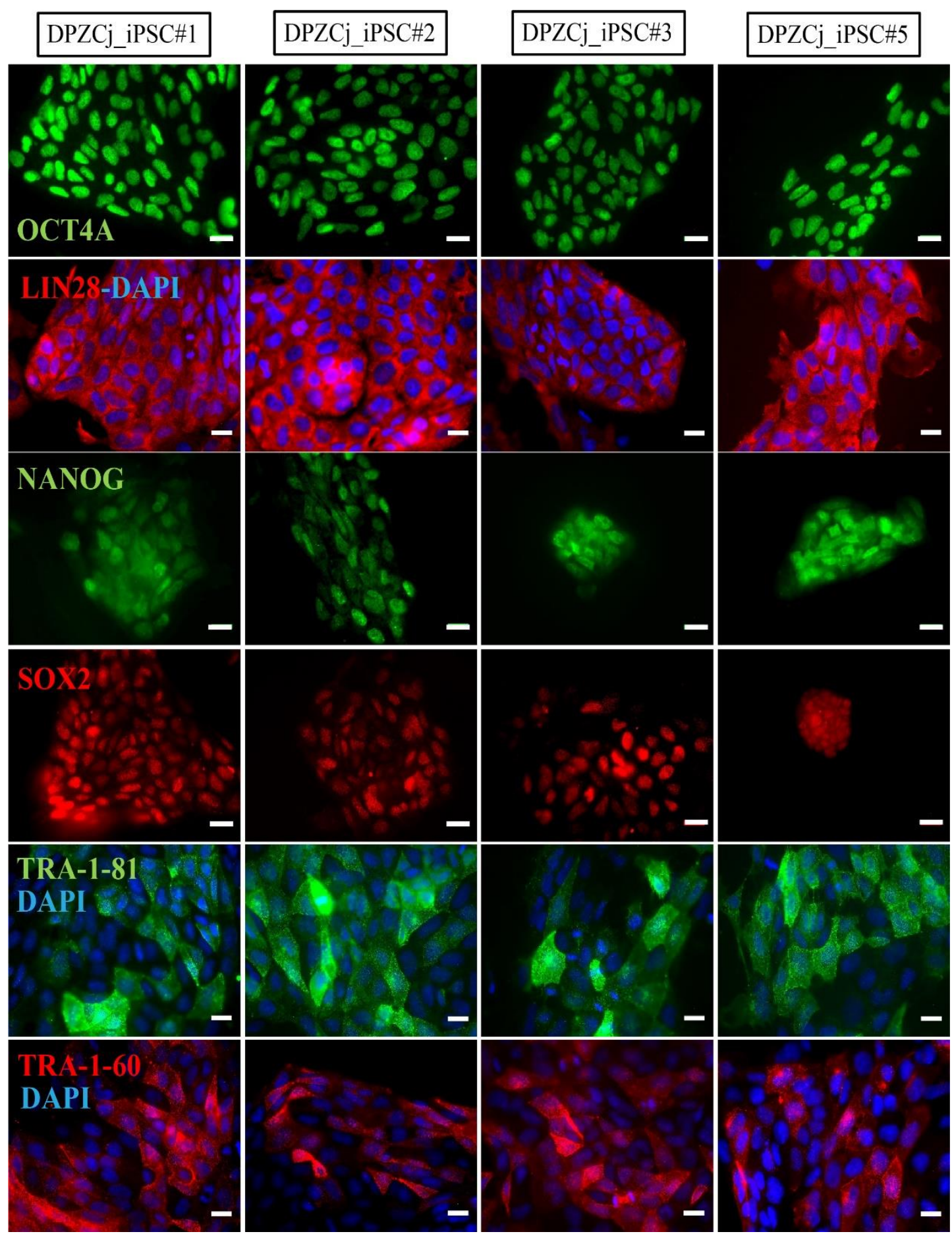

Suppl. Fig 4: Marmoset iPSC characterization by immunofluorescence. Detection of the pluripotency

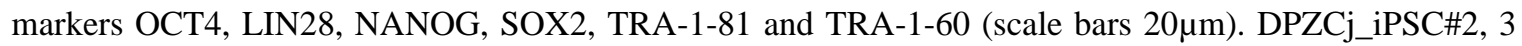
and 5 are transgene-free so all the expression detected is endogenous. DPZCj_iPSC\#1 still contains the plasmids; therefore, the expression comes from both, endogenous and exogenous expression. 
(A)

\begin{tabular}{|c|c|c|c|c|c|}
\hline & \multicolumn{5}{|c|}{ Exon size (bp) } \\
\hline & $<100$ & $100-200$ & $201-300$ & $301-400$ & $>400$ \\
\hline No of exons & 116 & 50 & 141 & 35 & 21 \\
\hline Percentage (\%) & 31.95 & 13.77 & 38.84 & 9.64 & 5.78 \\
\hline
\end{tabular}

(B)

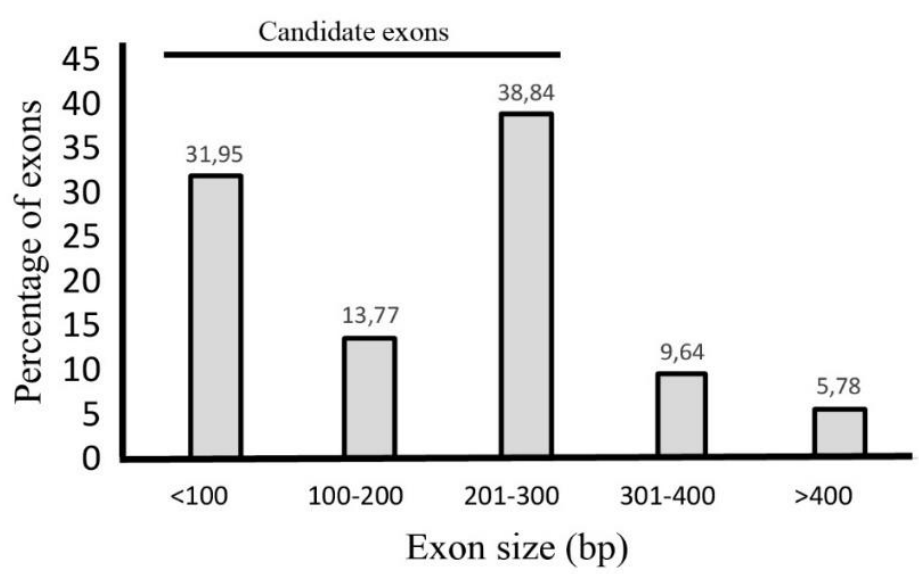

Suppl. Fig 5: (A) (B) Distribution of TTN exons according to their size, in base pairs (bp). Defined size rages, smaller than 100bp (<100), between 100 and 200bp (100-200), between 201 and 300bp (201-300), between 301 and 400b`p (301-400), and bigger than $400 \mathrm{bp}(>400)$.

\begin{tabular}{|c|c|c|c|c|c|}
\hline & Gene & $\begin{array}{l}\text { No. } \\
\text { Exons }\end{array}$ & Accession number & $\begin{array}{c}\text { No. } \\
\text { Symmetric } \\
\text { exons }\end{array}$ & $\begin{array}{c}\text { Percentage } \\
\text { of } \\
\text { symmetric } \\
\text { exons }(\%)\end{array}$ \\
\hline \multirow{5}{*}{$\begin{array}{l}\text { Thin filament } \\
\text { proteins }\end{array}$} & $\begin{array}{l}\text { Cardiac troponin } \mathrm{T} \\
\quad(T N N T 2)\end{array}$ & 16 & ENST00000509001.5 & 8 & 50 \\
\hline & $\begin{array}{l}\text { Cardiac troponin I } \\
\quad(T N N I 3)\end{array}$ & 8 & ENST00000509001.5 & 5 & 62.5 \\
\hline & $\begin{array}{l}\text { Cardiac troponin } \mathrm{C} \\
\quad(T N N C I)\end{array}$ & 6 & ENST00000232975.7 & 1 & 16.66 \\
\hline & $\alpha$-Tropomyosin (TPMI) & 9 & ENST00000358278.7 & 2 & 22.22 \\
\hline & $\alpha$-Cardiac actin $(A C T C 1)$ & 7 & ENST00000358278.7 & 2 & 28.57 \\
\hline \multirow{4}{*}{$\begin{array}{l}\text { Thick filament } \\
\text { proteins }\end{array}$} & $\begin{array}{c}\beta \text {-Myosin heavy chain } \\
(\text { (MYH7) }\end{array}$ & 40 & ENST00000290378.5 & 15 & 37.5 \\
\hline & $\begin{array}{l}\text { Ventricular regulatory } \\
\text { light chain (MYL3) }\end{array}$ & 6 & ENST00000395869.5 & 3 & 50 \\
\hline & $\begin{array}{l}\text { Ventricular esential light } \\
\text { chain (MYL2) }\end{array}$ & 7 & ENST00000228841.12 & 4 & 57.14 \\
\hline & $\begin{array}{l}\text { Cardiac myosin-bining } \\
\text { protein } \mathrm{C}(M Y B P C 3)\end{array}$ & 35 & ENST00000545968.5 & 14 & 40 \\
\hline \multirow{4}{*}{$\begin{array}{l}\text { Titin and Z-disc } \\
\text { proteins }\end{array}$} & Titin $(T T N)$ & 363 & ENST00000589042 & 311 & 85.67 \\
\hline & T-cap $(T C A P)$ & 2 & ENST00000309889.2 & 1 & 50 \\
\hline & MLP (CSRP3) & 7 & ENST00000533783.2 & 0 & 0 \\
\hline & Myozenin-2 (MYOZ2) & 6 & ENST00000307128.5 & 2 & 33.33 \\
\hline
\end{tabular}




\begin{tabular}{c|ccccc} 
& $\alpha$-Actinin $(A C T N 2)$ & 23 & ENST00000546208.5 & 13 & 56.52 \\
& Obscurin $($ OBSCN $)$ & 116 & ENST00000570156.6 & 84 & 72.41 \\
\hline $\begin{array}{c}\text { Citoplasmic } \\
\text { protein }\end{array}$ & Cypher $(L D B 3)$ & 14 & ENST00000429277.6 & 1 & 7.14 \\
\hline & Dystrophin $(D M D)$ & 79 & ENST00000357033.8 & 40 & 50.63
\end{tabular}

Suppl. Table 3: Cardiac relevant genes quantification of symmetric exons.

\begin{tabular}{|c|c|c|c|c|}
\hline Transcript & $\begin{array}{l}\text { No. } \\
\text { exons }\end{array}$ & Accession number & $\begin{array}{l}\text { No. Symmetric } \\
\text { exons }\end{array}$ & Percentage of symmetric exons (\%) \\
\hline Meta & 363 & ENST00000589042 & 311 & 85.67493113 \\
\hline N2BA & 313 & ENST00000591111 & 261 & 83.38658147 \\
\hline $\mathrm{N} 2 \mathrm{~B}$ & 191 & ENST00000460472 & 142 & 74.34554974 \\
\hline $\mathrm{N} 2 \mathrm{~A}$ & 312 & ENST00000342992 & 261 & 83.65384615 \\
\hline Novex-1 & 191 & ENST00000359218 & 144 & 75.39267016 \\
\hline Novex-2 & 192 & ENST00000342175 & 143 & 74.47916667 \\
\hline Novex-3 & 46 & ENST00000360870 & 31 & 67.39130435 \\
\hline CRONOS & 124 & & 97 & 78.22580645 \\
\hline
\end{tabular}

Suppl. Table 4: Symmetric exon quantification in the mayor TTN isoforms.

\begin{tabular}{|c|c|c|c|c|c|c|}
\hline $\begin{array}{c}\text { Exon } \\
\mathbf{N}^{\mathbf{0}} \\
\end{array}$ & Exon ID & Length & Location & Domains & $\begin{array}{l}\text { Exon } \\
\text { Phase } \\
\end{array}$ & $\begin{array}{c}\text { Exon } \\
\text { PSI } \\
\end{array}$ \\
\hline 3 & ENSE00003807606 & 204 & Z-disk & Ig-like 1 & 1 & 1.00 \\
\hline 4 & ENSE00003810976 & 288 & Z-disk & Ig-like 2 & 1 & 1.00 \\
\hline 8 & ENSE00003803639 & 153 & Z-disk & Z-repeat 1 & 0 & 1.00 \\
\hline 9 & ENSE00003810843 & 138 & Z-disk & Z-repeat 2 & 0 & 1.00 \\
\hline 10 & ENSE00003803899 & 126 & Z-disk & Z-repeat 3 & 0 & 1.00 \\
\hline 13 & ENSE00003805623 & 138 & Z-disk & Z-repeat 6 & 0 & 0.96 \\
\hline 14 & ENSE00003801659 & 294 & Z-disk & Z-repeat 7 & 0 & 1.00 \\
\hline 15 & ENSE00003804455 & 123 & Z-disk & & 0 & 0.99 \\
\hline 16 & ENSE00003810306 & 282 & $\begin{array}{c}\text { near Z- } \\
\text { disk }\end{array}$ & & 0 & 0.99 \\
\hline 17 & ENSE00003805296 & 66 & $\begin{array}{c}\text { near Z- } \\
\text { disk }\end{array}$ & & 0 & 0.99 \\
\hline 20 & ENSE00003804962 & 216 & $\begin{array}{c}\text { near Z- } \\
\text { disk }\end{array}$ & Ig-like 4 & 2 & 1.00 \\
\hline 23 & ENSE00003807963 & 234 & $\begin{array}{c}\text { near Z- } \\
\text { disk }\end{array}$ & Ig-like 5 & 0 & 1.00 \\
\hline 26 & ENSE00003811446 & 165 & $\begin{array}{c}\text { near Z- } \\
\text { disk }\end{array}$ & Ig-like 6 & 1 & 1.00 \\
\hline 29 & ENSE00003809440 & 282 & I-band & Ig-like 11 & 1 & 1.00 \\
\hline 30 & ENSE00003802960 & 267 & I-band & Ig-like 12 & 1 & 1.00 \\
\hline 31 & ENSE00003804241 & 273 & I-band & Ig-like 13 & 1 & 1.00 \\
\hline 32 & ENSE00003806988 & 264 & I-band & Ig-like 14 & 1 & 1.00 \\
\hline
\end{tabular}




\begin{tabular}{|c|c|c|c|c|c|c|}
\hline 33 & ENSE00003804968 & 261 & I-band & & 1 & 1.00 \\
\hline 34 & ENSE00003809850 & 261 & I-band & Ig-like 15 & 1 & 1.00 \\
\hline 35 & ENSE00003806479 & 264 & I-band & & 1 & 1.00 \\
\hline 36 & ENSE00003804523 & 261 & I-band & & 1 & 1.00 \\
\hline 37 & ENSE00003807378 & 261 & I-band & Ig-like 16 & 1 & 1.00 \\
\hline 38 & ENSE00003807271 & 261 & I-band & Ig-like 17 & 1 & 1.00 \\
\hline 42 & ENSE00003802092 & 285 & I-band & Ig-like 19 & 1 & 1.00 \\
\hline 43 & ENSE00003807103 & 126 & I-band & Ig-like 20 & 1 & 1.00 \\
\hline 44 & ENSE00003801355 & 189 & I-band & Ig-like 20 & 1 & 1.00 \\
\hline 47 & ENSE00003810791 & 57 & I-band & & 1 & 1.00 \\
\hline 49 & ENSE00003805227 & 279 & I-band & Ig-like 24 & 1 & 1.00 \\
\hline 219 & ENSE00003804802 & 81 & I-band & PEVK27 & 1 & 1.00 \\
\hline 220 & ENSE00003811452 & 75 & I-band & PEVK28 & 1 & 1.00 \\
\hline 221 & ENSE00003807205 & 90 & I-band & & 1 & 1.00 \\
\hline 222 & ENSE00003806747 & 63 & I-band & PEVK29 & 1 & 1.00 \\
\hline 223 & ENSE00003810755 & 90 & I-band & PEVK30 & 1 & 1.00 \\
\hline 226 & ENSE00003803996 & 279 & I-band & Ig-like 81 & 1 & 1.00 \\
\hline 227 & ENSE00003807976 & 276 & I-band & Ig-like 82 & 1 & 1.00 \\
\hline 230 & ENSE00003811410 & 264 & I-band & & 1 & 1.00 \\
\hline 231 & ENSE00003805150 & 267 & I-band & Ig-like 83 & 1 & 1.00 \\
\hline 232 & ENSE00003801907 & 264 & I-band & Ig-like 84 & 1 & 1.00 \\
\hline 235 & ENSE00003811384 & 267 & I-band & Ig-like 85 & 1 & 1.00 \\
\hline 236 & ENSE00003801954 & 267 & I-band & & 1 & 1.00 \\
\hline 237 & ENSE00003809996 & 267 & I-band & Ig-like 86 & 1 & 1.00 \\
\hline 245 & ENSE00003811387 & 267 & I-band & Ig-like 90 & 1 & 1.00 \\
\hline 246 & ENSE00003802653 & 267 & I-band & Ig-like 91 & 1 & 1.00 \\
\hline 247 & ENSE00003802540 & 279 & I-band & Ig-like 92 & 1 & 1.00 \\
\hline 250 & ENSE00003807647 & 267 & I-band & & 1 & 1.00 \\
\hline 251 & ENSE00003802353 & 270 & I-band & Ig-like 95 & 1 & 1.00 \\
\hline 256 & ENSE00003809985 & 285 & A-band & & 1 & 1.00 \\
\hline 261 & ENSE00003809522 & 288 & A-band & Ig-like 96 & 1 & 1.00 \\
\hline 262 & ENSE00003804879 & 297 & A-band & Fibronectin type-III 6 & 1 & 1.00 \\
\hline 265 & ENSE00003806136 & 300 & A-band & Fibronectin type-III 8 & 1 & 1.00 \\
\hline 266 & ENSE00003804290 & 300 & A-band & Fibronectin type-III 9 & 1 & 1.00 \\
\hline 270 & ENSE00003808061 & 279 & A-band & Ig-like 97 & 1 & 1.00 \\
\hline 271 & ENSE00003811164 & 300 & A-band & $\begin{array}{c}\text { Fibronectin type-III } \\
12 \\
\end{array}$ & 1 & 1.00 \\
\hline 275 & ENSE00003809346 & 300 & A-band & $\begin{array}{l}\text { Fibronectin type-III } \\
15\end{array}$ & 1 & 1.00 \\
\hline 276 & ENSE00003806782 & 297 & A-band & $\begin{array}{l}\text { Fibronectin type-III } \\
16\end{array}$ & 1 & 1.00 \\
\hline 277 & ENSE00003804121 & 285 & A-band & Ig-like 99 & 1 & 1.00 \\
\hline 278 & ENSE00003804454 & 294 & A-band & $\begin{array}{l}\text { Fibronectin type-III } \\
17\end{array}$ & 1 & 1.00 \\
\hline 279 & ENSE00003803420 & 300 & A-band & $\begin{array}{c}\text { Fibronectin type-III } \\
18 \\
\end{array}$ & 1 & 1.00 \\
\hline 285 & ENSE00003801625 & 33 & A-band & Ig-like 101 & 0 & 1.00 \\
\hline
\end{tabular}




\begin{tabular}{|c|c|c|c|c|c|c|}
\hline 287 & ENSE00003804239 & 300 & A-band & $\begin{array}{c}\text { Fibronectin type-III } \\
22 \\
\end{array}$ & 1 & 1.00 \\
\hline 289 & ENSE00003801391 & 297 & A-band & Ig-like 102 & 1 & 1.00 \\
\hline 290 & ENSE00003806021 & 300 & A-band & $\begin{array}{l}\text { Fibronectin type-III } \\
24\end{array}$ & 1 & 1.00 \\
\hline 294 & ENSE00003808790 & 282 & A-band & Ig-like 103 & 1 & 1.00 \\
\hline 297 & ENSE00003807039 & 282 & A-band & Ig-like 104 & 1 & 1.00 \\
\hline 298 & ENSE00003804106 & 300 & A-band & $\begin{array}{l}\text { Fibronectin type-III } \\
29\end{array}$ & 1 & 1.00 \\
\hline 301 & ENSE00003807476 & 282 & A-band & Ig-like 105 & 1 & 1.00 \\
\hline 302 & ENSE00003807363 & 300 & A-band & $\begin{array}{l}\text { Fibronectin type-III } \\
32\end{array}$ & 1 & 1.00 \\
\hline 303 & ENSE00003808994 & 294 & A-band & $\begin{array}{c}\text { Fibronectin type-III } \\
33 \\
\end{array}$ & 1 & 1.00 \\
\hline 306 & ENSE00003805661 & 285 & A-band & Ig-like 109 & 1 & 1.00 \\
\hline 307 & ENSE00003804742 & 300 & A-band & $\begin{array}{l}\text { Fibronectin type-III } \\
42\end{array}$ & 1 & 1.00 \\
\hline 309 & ENSE00003801707 & 276 & A-band & Ig-like 110 & 1 & 1.00 \\
\hline 310 & ENSE00003810399 & 300 & A-band & $\begin{array}{c}\text { Fibronectin type-III } \\
44\end{array}$ & 1 & 1.00 \\
\hline 312 & ENSE00003802505 & 300 & A-band & $\begin{array}{c}\text { Fibronectin type-III } \\
46\end{array}$ & 1 & 1.00 \\
\hline 313 & ENSE00003806397 & 288 & A-band & Ig-like 111 & 1 & 1.00 \\
\hline 314 & ENSE00003807889 & 297 & A-band & $\begin{array}{l}\text { Fibronectin type-III } \\
47\end{array}$ & 1 & 1.00 \\
\hline 317 & ENSE00003807542 & 288 & A-band & & 1 & 1.00 \\
\hline 318 & ENSE00003810775 & 291 & A-band & $\begin{array}{l}\text { Fibronectin type-III } \\
50\end{array}$ & 1 & 1.00 \\
\hline 319 & ENSE00003811210 & 288 & A-band & $\begin{array}{l}\text { Fibronectin type-III } \\
51 \\
\end{array}$ & 1 & 1.00 \\
\hline 321 & ENSE00003808777 & 105 & A-band & $\begin{array}{l}\text { Fibronectin type-III } \\
52 \\
\end{array}$ & 1 & 1.00 \\
\hline 322 & ENSE00003806939 & 198 & A-band & $\begin{array}{l}\text { Fibronectin type-III } \\
53\end{array}$ & 1 & 1.00 \\
\hline 323 & ENSE00003811024 & 297 & A-band & $\begin{array}{l}\text { Fibronectin type-III } \\
54\end{array}$ & 1 & 1.00 \\
\hline 327 & ENSE00003802597 & 297 & A-band & $\begin{array}{l}\text { Fibronectin type-III } \\
99\end{array}$ & 1 & 1.00 \\
\hline 330 & ENSE00003806587 & 297 & A-band & $\begin{array}{l}\text { Fibronectin type-III } \\
102\end{array}$ & 1 & 1.00 \\
\hline 331 & ENSE00003807681 & 288 & A-band & Ig-like 130 & 1 & 1.00 \\
\hline 332 & ENSE00003811102 & 300 & A-band & $\begin{array}{l}\text { Fibronectin type-III } \\
103\end{array}$ & 1 & 1.00 \\
\hline 336 & ENSE00003811205 & 294 & A-band & $\begin{array}{l}\text { Fibronectin type-III } \\
110\end{array}$ & 1 & 1.00 \\
\hline 337 & ENSE00003808021 & 288 & A-band & Ig-like 133 & 1 & 1.00 \\
\hline 338 & ENSE00003804355 & 300 & A-band & $\begin{array}{c}\text { Fibronectin type-III } \\
111 \\
\end{array}$ & 1 & 1.00 \\
\hline 342 & ENSE00003801213 & 291 & A-band & Ig-like 136 & 1 & 1.00 \\
\hline 343 & ENSE00003809128 & 297 & A-band & $\begin{array}{l}\text { Fibronectin type-III } \\
119\end{array}$ & 1 & 1.00 \\
\hline 346 & ENSE00003809946 & 282 & A-band & Ig-like 137 & 1 & 1.00 \\
\hline 348 & ENSE00003802641 & 288 & A-band & Ig-like 138 & 1 & 1.00 \\
\hline
\end{tabular}


Chapter 4: An iPSC-based preselection platform for disease-inducing genetic modifications in non-human primates

\begin{tabular}{|c|c|c|c|c|c|c|}
$\mathbf{3 4 9}$ & ENSE00003806194 & 300 & A-band & $\begin{array}{c}\text { Fibronectin type-III } \\
124\end{array}$ & 1 & 1.00 \\
\hline $\mathbf{3 5 4}$ & ENSE00003810204 & 300 & A-band & $\begin{array}{c}\text { Fibronectin type-III } \\
129\end{array}$ & 1 & 1.00 \\
\hline
\end{tabular}

Suppl. Table 5: List of candidate exons for therapeutic removal. Exons were selected from the TTN metatranscript (ENST00000589042) and filtered according to symmetry, conservation between the different isoforms (PSI $>0.9)$ and size $(\leq 300 \mathrm{bp})$. 


\section{Discussion}

To accurately predict the effect of in vivo applications of stem cell therapeutics and to study human disease, utilization of animal models resembling patient phenotypes is necessary ${ }^{78}$. Such model organisms will enhance CVD specific research aiming at the establishment of novel therapies. Especially regarding preclinical trials, NHP play an important role and have a higher predictive value compared to rodent animal models ${ }^{8}$. Hence, even considering the practical and ethical difficulties accompanying these models, it is worth to further establish NHP models for biomedical research. Refining methods and shedding light into species-specific particularities of NHP will further improve the panel of available models for preclinical studies.

The first aim of this Ph.D. thesis was to refine methodologies for NHP-iPSC generation. Establishment of robust reprogramming protocols will allow exploration of CV systemrelated therapies in vitro and in vivo. With this aim, we have tested different reprogramming methods and generated a robust work-flow for iPSC generation from four different primate species. Additionally, we translate cardiomyocyte differentiation protocols to the particularities of these cells.

The second aim was to mimic human CVD in vitro using genome editing technologies in NHP-iPSC. We generated a platform that allows evaluation of induced genetic modifications using species-specific iPSC. The platform will be crucial for future generation of genetically modified NHP. It may help to save resources, refine and reduce animal experiments and establish a relevant panel of NHP disease models.

Last, based on the study of sarcomeric protein homology between different species, we propose a CRISPR based therapeutic approach for dilated titin mutation-induced cardiomyopathy (DCM). Our initial contribution to the emerging field of myoediting by symmetric exon deletion from the $T T N$ gene needs significant experimental improvement and may complement the already existing approaches to treat monogenic cardiovascular diseases.

\section{Refinement of NHP-iPSC generation and culture}

Cell replacement therapies using versatile PSC made in the last years the way to the clinics ${ }^{127}$. Nevertheless, there are still many open questions, which can be addressed using NHP models. Species-specific cell types generated by NHP-iPSC differentiation can contribute for testing stem cell-based therapeutics in highly predictive coherent models ${ }^{92}$. Hence, generation of NHP-iPSC with similar quality as human iPSC is necessary. Furthermore, the limited number of available NHP-PSC lines as well as inter cell line variability, underline the importance of standardized and efficient NHP iPSC line generation protocols ${ }^{42}$.

Robust protocols for NHP-iPSC generation are currently unavailable. Reprogramming of cells from only a few primate species, e.g., macaques, have been achieved following the high standards for human iPSC generation ${ }^{128}{ }^{129}$. In contrast, other primate species like 
marmoset or baboon seem to be more resistance to reprogramming and, therefore, the respective protocols still need to be adapted ${ }^{130}$. The difficulties in generating NHP-iPSC is, amongst others, reflected in the high variability and discrepancies between studies using stem cells for regenerative purposes ${ }^{42}$. Chong and colleagues evaluated the regeneration of macaque myocardium using human ESC as source of transplanted cells 8586 . Shiba et al., on the other hand, used species-specific iPSCs to regenerate cynomolgus monkey myocardium, using, however, only one cell line ${ }^{92}$. This could result in studies biased by selection of highly specific cell lines and questions reproducibility of the studies. Therefore, improvement and standardization of NHP-iPSC generation will significantly impact on many research areas. Furthermore, this may contribute to the establishment of additional NHP species as models for translational research. Speciesspecific iPSC will further allow scientists to choose the adequate model according to their specific research interests and requirements.

In the present study, we refined and improved methods for NHP fibroblast reprogramming (Fig. 4). The starting point was a publication by Debowski et al. (2015), which demonstrated the possibility of neonatal marmoset fibroblast reprogramming using the piggyBac system. The piggyBac transposon stably delivered six reprogramming factors facilitating efficient iPSC generation, while the transposon was still present in the cells ${ }^{59}$. This study was used as a starting point for protocol optimization with the aims of increased efficiency of the generation process and improved quality of the resulting iPSC. The resulting protocols were further tested in three other primate species. We have explored two delivery mechanisms for the reprogramming factors, the piggyBac ${ }^{59}$, and an episomal vector system ${ }^{52}$. Both systems are DNA based and contain six (mSOX2, $\mathrm{mOCT4}, \mathrm{mKLF4}, \mathrm{mC}-M Y C, \mathrm{mLIN} 28$, and mNanog) or five (hOCT3/4, $\mathrm{hL}-M Y C, \mathrm{~h} L I N 28$,

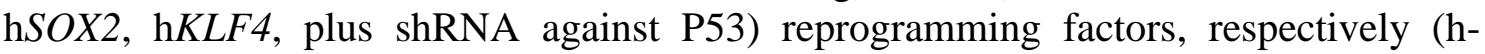
Human, and m-marmoset factors) (Fig. 4).

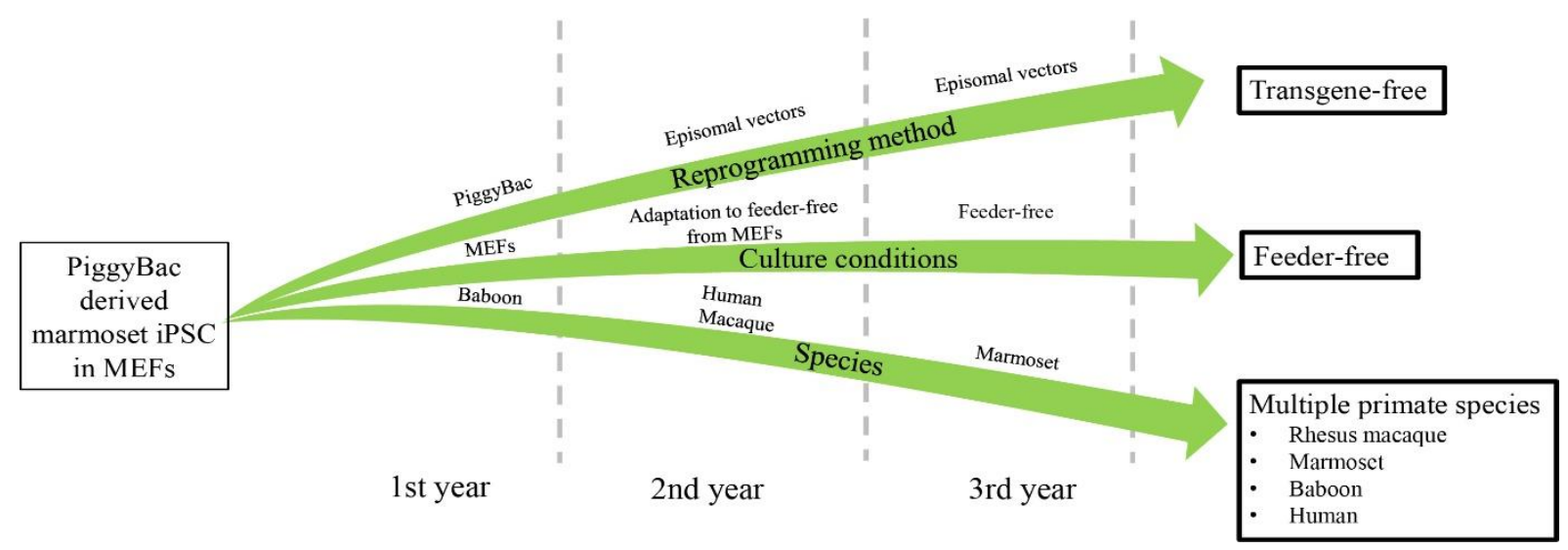

Figure 4: Schematic representation of aims in iPSC generation and maintenance achieved during the three years of the Ph.D. project. Each arrow represents one of the aims: 1-reprogramming method resulting in transgene-free cells, 2-culture conditions without xenogenic feeder cells, and 3-applicability of the protocols to different primate species with preclinical relevance.

First, the published piggyBac protocol was applied to rhesus macaque and baboon fibroblasts ${ }^{59}$. Only minor modifications were necessary for successful reprogramming, namely adjustment of culture conditions and colony splitting protocols. Rhesus, baboon and marmoset piggyBac derived iPSCs were initially maintained on MEFs, however, as a first approximation to feeder-free culture systems, we adapted them a posteriori to feeder-free conditions using commercially available medium and culture dish coating for 
human iPSCs (Chapter 1 and 3) 60 131. Feeder-free cultivation was successful for all three species, however not for all lines tested. Furthermore, the iPSCs showed limited differentiation and low proliferation in the new culture conditions. Hence, the protocols for maintenance needed adjustment. We suspected that the long term culture of the iPSCs on MEF impaired the plasticity of the cell lines. Furthermore, we hypothesize that differences between NHP- and human-iPSC require the development specific culture conditions for NHP-iPSC. Another disadvantage of this protocol was the insertion of the reprogramming construct (permanently Chapter 1 or transiently Chapter 3 ) in the genome. This could lead to mutagenesis, chromosomal abnormalities, failing differentiation and make the generated iPSCs useless for transplantation studies ${ }^{39}$. To overcome the integration problem, we decided to test episomal vector reprogramming for all three NHP species. Episomal vector derived iPSC were cultured in feeder-free conditions from the beginning of reprogramming using commercial medium developed for human PSC.

Using the episomal vector system containing the human factors published by Okita et al., we were able to reprogram human, baboon, rhesus and marmoset fibroblasts ${ }^{52}$ (Chapter 2 and 4). Even though primary colonies appeared with morphology indicating pluripotency of the cells in the primary plates after transfection, we faced the problem of maintaining these cells. Commercially available media led to NHP-iPSC instability and ultimately to differentiation. Hence, we tested different media compositions in order to generate a universal primate iPSC culture medium. Testing different compounds the Universal primate pluripotent stem cell (UPPS) medium was empirically developed (Chapter 2). Based on StemMACS ${ }^{\text {TM }}$ iPS-Brew XF in combination with one Wnt pathway inhibitor (IWRI) and one activator (Chir99021), our culture conditions represent a suitable medium for rhesus, human and baboon iPSCs culture. Unfortunately, marmoset iPSCs differentiated under these conditions. During the last year, we focused on finetuning of the UPPS medium to be able to culture all four primate species relevant in biomedical research. The addition of Activin A was the key to stabilize also marmoset PSCs (Chapter 4). Even though Activin A proved to be necessary for marmoset iPSC stability, the proliferation rate was affected negatively. To enhance proliferation, we added Leukemia inhibitory factor (LIF) in high concentration $(10 \mathrm{ng} / \mathrm{ml})$. We named the new formulation UPPS advanced (UPPS-Adv). This medium is able to stabilize beside marmoset iPSCs also rhesus, baboon and human iPSCs. Regardless of the addition of LIF, the proliferation rates were yet lower in comparison with UPPS. However, the use of UPPS-Adv is required only during the first passages of iPSC line establishment. In conclusion, we recommend using UPPS-Adv during NHP-iPSC establishment and switching to UPPS for further maintenance and experimental approaches (Chapter 4).

NHP-iPSC generated using episomal vectors and maintained in the novel UPPS/UPPSAdv media were fully characterized, and the absence of the episomal vectors was confirmed for most of the newly generated iPSC lines. Taken together, we were able to generate stable transgene-free, feeder-free NHP-iPSC from all four primate species tested.

\subsection{Characterization of NHP-iPSCs}

In order to confirm the pluripotent stem cell identity of the generated lines, we performed an in-depth characterization. Human iPSCs used for cell-based therapeutics require clinical-grade quality. Furthermore, robust and reproducible directed differentiation protocols guarantee efficacy and safety of the therapeutic approach for the patients (GMP- 
compliance) ${ }^{132}{ }^{133}$. Hence, it was necessary to reach a consensus for the analysis required to consider iPSC "clinical grade". This has been achieved in the last years following the guidance of institutions like the International Stem Cell Banking Initiative and by a series of workshops ${ }^{64}$. For NHP, the standards are more diffuse, involving only few research groups trying to define the required minimum of analyses ${ }^{42}$. In order to improve the current situation, we decided to establish and follow systematically a work-flow that allows us to ensure the quality of the cell lines generated ${ }^{42}$. As mentioned, gold-standards for human are well-defined; hence we adopted these standards for our non-human primate iPSCs. We additionally added few tests required for proper evaluation of NHP cells (Fig. $5)$.

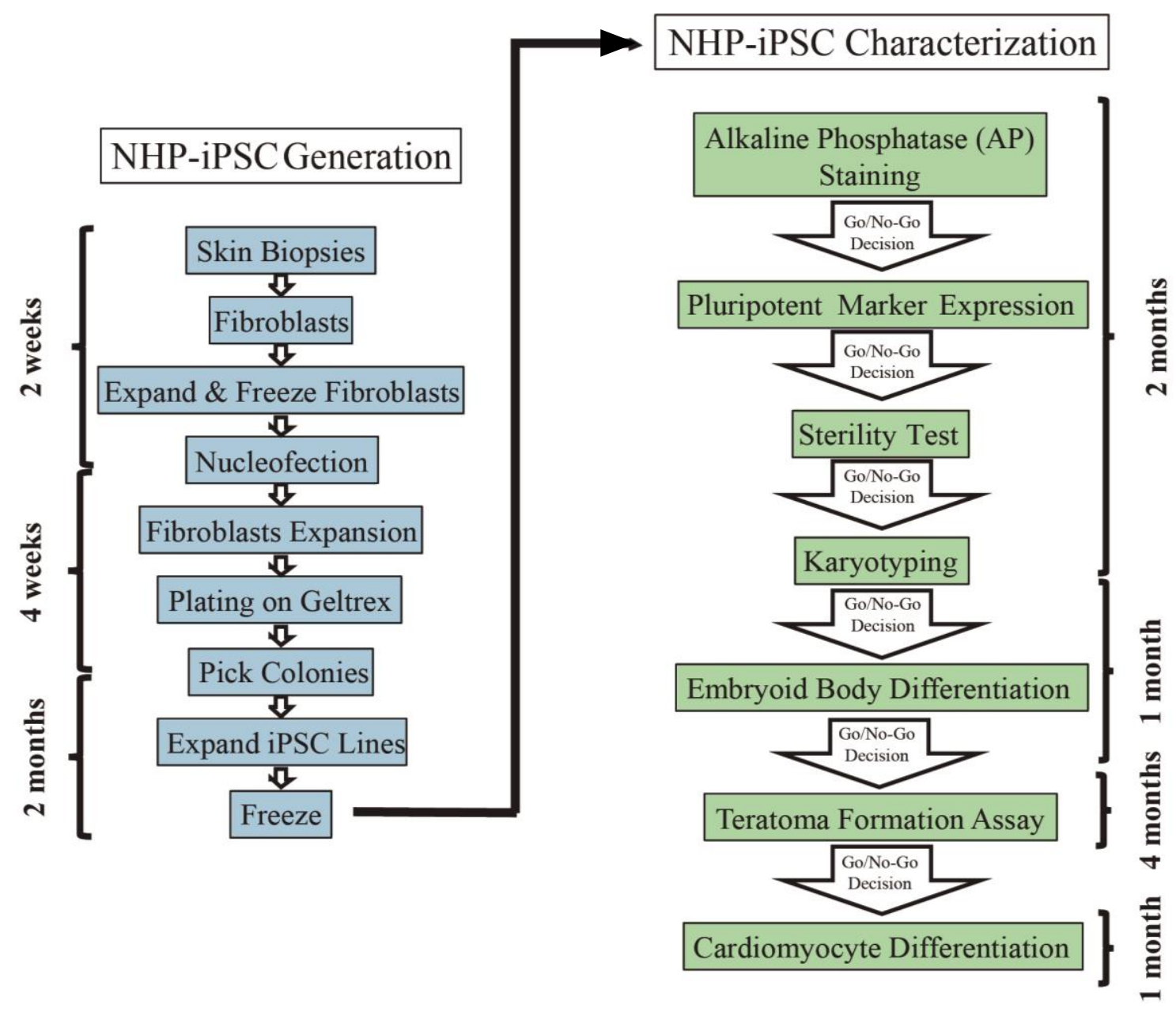

Figure 5: Work-flow for the generation and characterization of NHP-iPSCs generated in this project. The estimated duration of each step is indicated (Total duration 1-1,5 years per line). Figure modified from ${ }^{42}$.

Every step of the characterization was considered as a go/no go decision, only allowing us to proceed to the next steps in case positive results during the previous criterion. First, we performed a morphological characterization of the cells and colonies. Only lines showing a typical PSC morphology were considered for further characterization. Then, as a preliminary characterization in early passages (10/15) alkaline phosphatase activity was evaluated. Subsequently, the expression of canonical pluripotency markers on the protein and mRNA level was analyzed. Only lines expressing a specific set of pluripotency markers endogenously were further studied. Next, sterility tests were performed to ensure the absence of selected viruses and mycoplasms (data not shown in this dissertation). This is particularly important regarding the potential preclinical 
application of the cell lines for transplantation. Mycoplasma test was run every six months, and additionally, the cells were tested for SIV, SRV, STLV, and CeHV-1 viruses ${ }^{41}$. SIV testing is crucial in the case of rhesus macaque and baboon iPSCs since this virus could impact on the outcome of the experiments and, more importantly, it entails a risk for the researcher. Careful karyotyping is an analysis that scientists sometimes overlook. Chromosomal alterations may occur during the process of reprogramming and depending on the kind of mutation, affect the phenotype of the cell line drastically ${ }^{134}$. We karyotyped the human, macaque, and baboon lines in collaboration with Prof. Bartels (Institute of Human Genetics, UMG Göttingen).

Once the molecular identity of the different lines has been proven, pluripotency needs to be tested also on a functional level. We performed both in vitro and in vivo spontaneous differentiation assays in order to assess the pluripotent status of the cells. Teratoma formation assay, performed in collaboration with Prof. Dressel (Institute of Cellular and Molecular Immunology, UMG Göttingen), was exceptionally informative. While getting information on in vivo pluripotency, it also provides information about tumorigenicity of the lines. Finally, as we aim to use our cells in the context of CV research, we tested the capacity of the cells to differentiate into cardiomyocytes in vitro.

Altogether approximately one and a half years of work are required to generate and characterize one line according to our self-set standards. Even though this process is laborious and time consuming, we consider it crucial to ensure the quality of the iPSC lines generated before they are used in (in vivo) downstream applications (Fig. 5).

\section{Efficient generation of functional NHP-iPSC cardiomyocytes}

After the generation of state of the art primate iPSCs, we tested different protocols to direct these cells towards cardiomyocyte-like cells. Future stem cell-based regenerative therapies of the myocardium will require large amounts of iPSC-CM. Therefore, efficient and fine-tuned protocols need to be established ${ }^{135}$. Based on the protocol developed by Tiburcy and collaborators ${ }^{84}$, Dr. Stauske was able to adapt this protocol to the generation of rhesus and baboon iPSCs-CM (Chapter 2) ${ }^{84}$. Results obtained with these species could then be successfully translated to marmoset and human iPSCs (Chapter 4). The protocol is based on the modulation of key elements of the $\mathrm{Wnt} / \beta$-catenin signaling pathways via small molecules and recombinant proteins. The differentiation can be divided into two phases, $-1^{\text {st }}$ an initial step to induce differentiation towards the mesodermal pathway (B27 Suppl minus Insulin, supplemented with Activin A, Chir99021, BMP4), and $-2^{\text {nd }}$ cardiomyocyte specification phase (IWRI, B-27 Suppl minus Insulin) (Chapter 2).

Using this work-flow, it was possible to reproducibly generate iPSC-CM from all four primate species, while the human-specific protocol was not sufficient to induce CM differentiation reproducibly in all cell lines (Suppl. Video 1 macaque and 2 marmoset). Differentiation efficiency differed between species and cell lines and was accurately evaluated by FACS. The differences in the efficiency were overcome via metabolic selection of the CM resulting in almost pure iPS-CM cultures ${ }^{136}$. Previous reports have shown CM-differentiation in macaque and marmoset 92137 138. To our knowledge, however, we are the first who successfully applied a universal CM differentiation protocol to different primate species. Furthermore, to the present, there are no reports of baboon PSC derived cardiomyocytes. Additionally, marmoset ESC- derived cardiomyocytes 
have been generated, however using protocols that do not allow the mass production of differentiated cells ${ }^{138}$.

Basic molecular characterization of the iPSC-CM was performed in the protein level by demonstrating the expression of specific cardiac markers (Chapter 2 and 4). In order to initially characterize the generated beating iPSC-CM functionally, we performed electrophysiological characterization of the generated cells using 2D (Microelectrode Array, MEA) and 3D (Engineered heart muscle, EHM) systems 84139140 . The generated iPSCs-CM showed inotropic responses, reacting to isoprenaline, propranolol, and increased $\mathrm{Ca}^{2+}$ levels. EHM measurements are particularly relevant since this approach is able to analyze organotypic responses and to improve the maturation of the generated cells (proved for human iPSC in ${ }^{84}$ ). Altogether, the generated NHP-iPSC-CM appear to be functional in different assays. These findings encourage their use in preclinical in vivo applications aiming at myocardial regeneration.

\section{Modeling cardiovascular disease in NHP-iPSCs}

To the present, NHP served as CVD models based on surgical and drug-induced approaches ${ }^{8}$. Generation of transgenic NHP CVD models will allow the analysis of (human) disease with reduced heterogeneity between studies. Also, by the introduction of disease-relevant mutations in NHP the emergence and progression of the disease can be studied, which is in contrast to most conventional disease models. Furthermore, the genetically induced disease can be inherited to the offspring allowing cross-generation, longitudinal studies. These NHP model organisms will be crucial for the understanding of human diseases and for the establishment of novel therapeutic approaches. Essential for this approach is the genetic modification of NHP embryos ${ }^{97}$.

Even though advances in genome editing technologies have allowed the generation of few genetically modified NHP (including macaques, cynomolgus monkeys, and marmosets), research is yet struggling to develop robust workflows 8142143144 . Given ethical and practical limitations in NHP in vivo studies (reviewed in Chapter 3, 4 in detail), we believe that robust in vitro evaluation systems for the introduction of genetic modifications is imperative. These platforms will allow assessing the efficiency and efficacy of induced genomic mutations in vitro before engaging in the in vivo project. Usually, researchers back their NHP studies with in vivo data from small animals models like mice. As mentioned before, these studies are an approximation, but not necessarily predictive of the outcome of the study using NHP ${ }^{99}$. Therefore we believe that is necessary to have a system to predict the molecular and cellular consequences of a genome modification in species-specific cells, before it is applied in vivo to modify an NHP.

We used our transgene- and feeder-free NHP-iPSC to develop a platform for the assessment of genome editing (Fig. 5). Utilizing all four species will be possible to evaluate species-specific responses to induced mutations in vitro. Furthermore, our finetuned CM-differentiation protocol may allow a preliminary assessment of the potential in vivo CVD phenotype. NHP-iPSC genome editing will provide information on efficiency and safety of the approach.

Only very few publications show genome editing and subsequent single-cell cloning of NHP-iPSC 97145 . In order to develop a robust protocol, we searched the literature for 
protocols generated for clonal expansion of human iPSC ${ }^{146}$. We aimed to develop efficient universal protocols for all primate species used in this study. The first step towards induction of defined mutations is the adjustment of single-cell cloning protocols. In the course of this Ph.D. project, single-cell dissociation, sorting, and cloning have been adjusted for NHP-PSC (Chapter 3 and 4). We were able to generate a robust approach that could be applied to all species tested here. Furthermore using the versatility of the piggyBac transposon system in combination with CRISPR/Cas editing technology, we were able to induce clinically relevant mutations in all PSC. This process was especially laborious due to the high number of publications defining human protocols and the discrepancies between them. Additionally, the relatively poor genomic annotation of NHP makes the design of the editing tools challenging ${ }^{8}$.

(A)

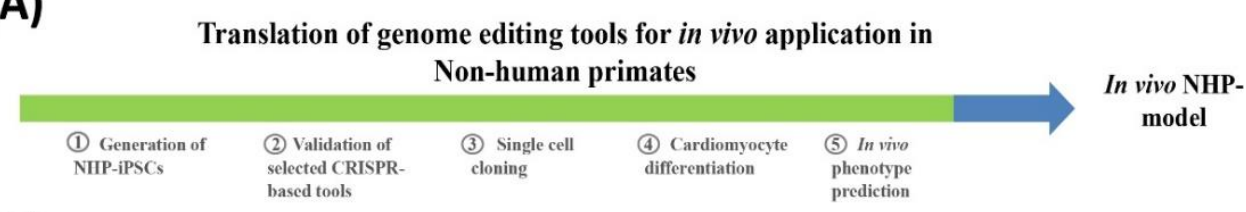

(B)

(1) Generation of

primate iPSCs
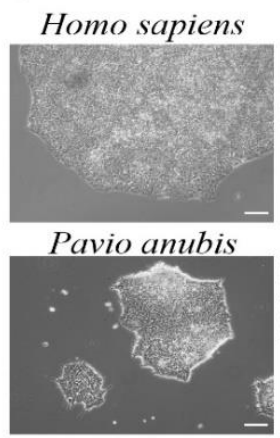

(C)

$$
\begin{aligned}
& \text { (2) Validation of } \\
& \text { selected CRISPR- } \\
& \text { based tools }
\end{aligned}
$$

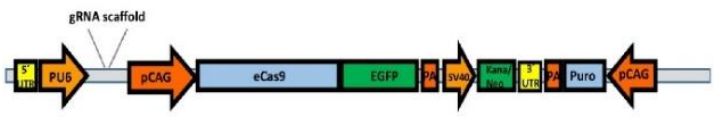

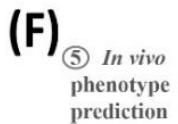

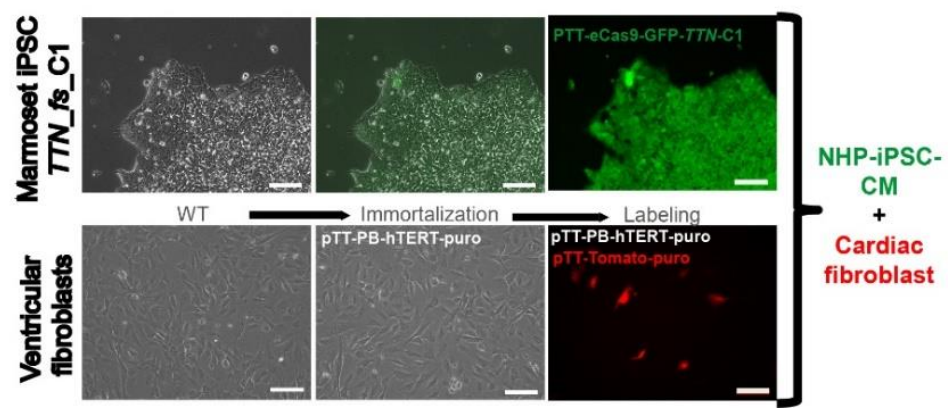

(D) (3) Single cell

Macaca mulatta
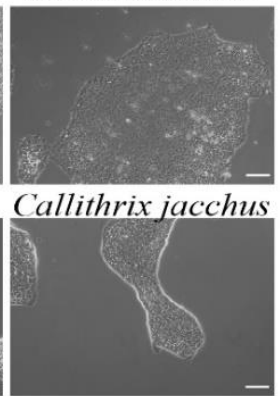

(E) (4) Cardiomyocyt differentiation

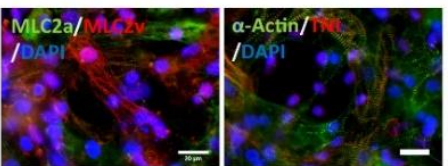

TTN/
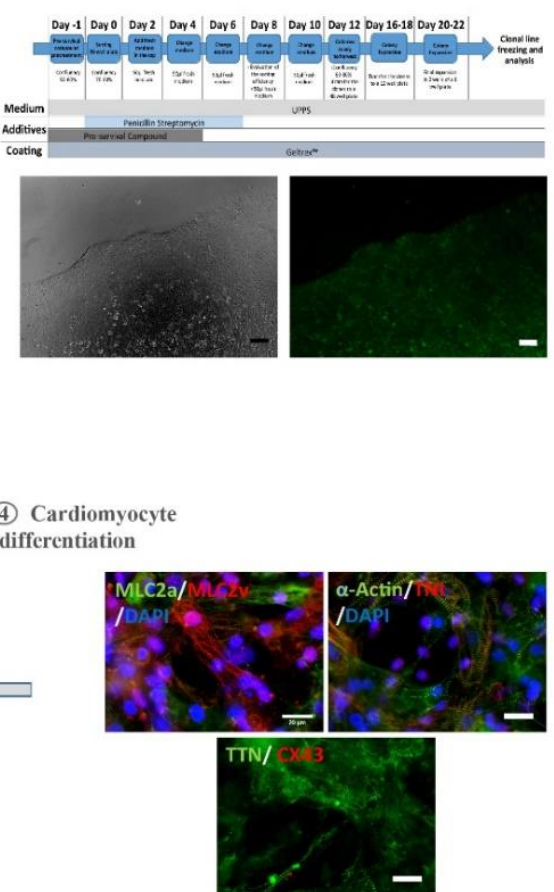
Figure 6: Proposed in vitro platform for the evaluation of genetic modifications for the generation of genetically modified NHP. (A) Schematic representation of the suggested work-flow with 5 distinctive phases: 1-generation of NHP-iPSC, 2-validation of the selected genome editing tools, 3-mutation induction and single cells cloning, 4- cardiomyocyte differentiation of the mutated iPSC, and 5-prediction of the in vivo phenotype. (B) Generated primate transgene- and feeder-free iPSC (Chapter 2, 3 and 4) (scale bars $100 \mu \mathrm{m}$ ). (C) CRISPR vector used for gRNA validation and mutation induction (Chapter 3). (D) Single-cell cloning protocol (upper pannel, Chapter 3 and 4), for the generation of genetically modified NHP monoclonal lines (lower panel). (E) Representative marmoset iPSC-CM (scale bars 20 $\mu \mathrm{m}$ ). (F) Schematic representation of the combination of species-specific iPSC containing clinically relevant mutations (expressing Cas9-GFP fusion transcript) and immortalized ventricular fibroblasts (RFP positive cells) (Chapter 4) (scale bars $100 \mu \mathrm{m})$.

We strived to establish an in vitro evaluation work-flow that will support in vivo approaches to the establishment of genetically modified monkeys. We believe that the present study will reduce the ethical problematics connected with the genetic modification of NHP research and contributes to the 3Rs (reduce, refine, and replace) of animal research. This approach will complement future in vivo project in the early phases. Additionally, by refining protocols in vitro, it will help to reduce the number of animals needed for in vivo studies.

\section{Exploring new therapies for monogenic cardiovascular diseases}

Dilated cardiomyopathy (DCM) represents one of the leading causes of death in CVD patients. DCM has a high incidence in the general population and is characterized by left ventricle enlargement and/or dysfunction. DCM demonstrates a complex etiology and has been diagnosed with accelerated rates in the last years ${ }^{147}$. In recent years, Next Generation Sequencing (NGS), together with detailed epidemiological data, allowed researchers to identify the genetic background of this disease. Mutations in up to 40 genes have been related to inherited DCM ${ }^{147}{ }^{148}$. These mutations affect proteins related to a broad range of cellular structures like nuclear envelope, cytoskeleton, and sarcolemma amongst others ${ }^{147}$. Within the mutated proteins, Titin (TTN) is particularly interesting as it is responsible for up to $25 \%$ of the familiar cases of DCM.

TTN, being the largest protein in the human body, is crucial in striated muscle. Here, the protein is responsible for sarcomere assembly and passive tension ${ }^{147} 148$. Studies in DCM have demonstrated truncation mutations in TTN as the most frequently occurring alteration in DCM patients. Myoediting approaches, relying on the reframing of the shifted, i.e. mutated open reading frame resulting in truncated proteins, have been recently introduced as a potential method of patient treatment. The approach describes the usage of CRISPR/Cas for correction of the disease-causing mutation in skeletal muscle and heart ${ }^{149}$. The therapeutic application of CRISPR/Cas in this context can be divided into four main groups. 1- Exon deletion ${ }^{150}{ }^{151}$, 2- exon skipping ${ }^{152} 153$, 3- exon reframing via targeted frameshift ${ }^{154}$ and 4- exon knock-in ${ }^{155}$. Exon deletion describes the deletion of mutation containing exons by targeting adjacent introns using two different gRNAs. Exon skipping strategies target the splice acceptor/donor sequence of the exon containing the mutation. Exon reframing induces a second mutation generating a second frameshift, restoring the protein reading frame. Finally, exon knock-in is the most elegant but at the same time challenging of all mentioned approaches. It involves the replacement of the mutated exon with a wildtype exon by HDR ${ }^{149}$. All these approaches have been tested in vitro and in vivo to correct a variety of CVDs. To the present, most studies exploring the 
different approaches focus on pathologies in which the disease-causing mutations are concentrated in hot-spots of a gene, e.g., DMD. For TTNtv-induced DCM the scenario is more complex, due to the broad range of locations of the mutations. Therefore, it is of utmost importance to test combinations of the mentioned approaches. This, in the end, might provide solutions for the treatment of most mutations and finally may result in causal disease treatment ${ }^{149}$.

In this study, we proposed a novel approach for DCM treatment (Chapter 4). The hypothesis is based on the extraordinarily high number of symmetric TTN exons. We hypothesized that the deletion of symmetric exons containing mutations can be used to overcome the DCM phenotype. By selecting the exons according to size, PSI, and symmetry, we have elaborated a library of candidate exons to test the approach. Furthermore, we have compiled a possible workflow for testing the hypothesis.

First experimental data have been collected in order to evaluate the proposed approach. TTN exon 280 has been deleted in one human iPSC line (Chapter 4) and evidence of successful deletion of this exon in other cell lines has been provided. Further, characterization of the phenotypic alterations of this line is necessary; however, we provided proof of concept of this approach.

For each one of the locations proposed, DCM exon deletion therapies need to be carefully evaluated. However, the progress described here strongly encourages to deeper test this approach using also additional target exons. We believe that this approach is particularly useful to treat truncating variants of TTN and may provide in the future a clear benefit for the respective DCM patients.

\section{Conclusions/Outlook}

I have focused in these collaborative efforts in the context of my Ph.D. project on the development of tools and protocols contributing to the establishment and application of NHP-iPSC. We aim to apply the generated lines in stem-cell-based regenerative projects for the cardiovascular system and for CVD disease modeling.

We were able to establish robust protocols for the generation and maintenance of iPSCs from four different primate species. All cell lines from the different species have been generated and characterized using one universal protocol. The developed protocol facilitates to go back and forth from NHP models to human, making possible direct translation of the results obtained using NHP. Furthermore, there are to date only few (or even none - depending on the species) publications describing the generation of NHPiPSC according to the standards set in this project.

The best way to evaluate the impact and value of any biotechnological tool is to assess its acceptance by the scientific community. Even though most results generated in this project have not been published yet, the generated NHP-iPSC are already used in different basic and preclinical projects. For instance, we are currently screening in collaboration with partners for AAV serotype specificity for the heart using NHP iPSC-CM. Additionally, the generated cells are being used in different evolutionary and developmental studies. Furthermore, already testing the potential of the generated cells to regenerate for instance the myocardium or the macula is currently tested. . 
Additionally, using the generated cells, we constructed to our knowledge the first NHPiPSC-based platform for the evaluation of genetic engineering approaches before their in vivo application. Generation of genetically modified NHP models is becoming a highly important field in biomedical sciences. Using the proposed work-flow it is possible to evaluate the accuracy and efficiency of a genome editing approach and may assist in the prediction the in vivo phenotype. Our main goals with this approach are (1) to contribute to the 3Rs inherent to NHP experimentation and (2) to support scientists in designing optimal approaches to the establishment of appropriate genetically modified NHP models. 


\section{References Introduction and Discussion}

1. Arnett, D. K. et al. 2019 ACC / AHA Guideline on the Primary Prevention of Cardiovascular Disease : Executive Summary. (2019).

2. Csöbönyeiová, M., Polák, Š. \& Danišovič, L. Toxicity testing and drug screening using iPSC-derived hepatocytes, cardiomyocytes and neural cells. Can J Physiol Pharmacol 94, 687-94 (2016).

3. Russell, J. C. \& Proctor, S. D. Small animal models of cardiovascular disease : tools for the study of the roles of metabolic syndrome, dyslipidemia, and atherosclerosis. Cardiovasc. Pathol. 15, 318-330 (2006).

4. Rogers, J. \& Gibbs, R. A. Comparative primate genomics: emerging patterns of genome content and dynamics Jeffrey. Nat Rev Genet. 15, 347-359 (2014).

5. Aglieri, V. \& Belin, P. A " voice patch" system in the primate brain for processing vocal information? Hear. Res. 366, 65-74 (2018).

6. Margulies, E. H. et al. Analyses of deep mammalian sequence alignments and constraint predictions for $1 \%$ of the human genome. Genome Res. 17, 760-774 (2007).

7. Li, T., Ai, Z. \& Ji, W. Primate stem cells : bridge the translation from basic research to clinic application. Sci China Life Sci. 61, 12-21 (2018).

8. Cox, L. A. et al. Nonhuman Primates and Translational Research Cardiovascular Disease. ILAR J 58, 235-250 (2017).

9. Camacho, P., Fan, H., Liu, Z. \& He, J. Large Mammalian Animal Models of Heart Disease. J. Cardiovasc. Dev. Dis. 30, 1-11 (2016).

10. Phillips, K. A. et al. Why Primate Models Matter. Am J Primatol. 76, 801-827 (2014).

11. Chellman, G. J. et al. Developmental and Reproductive Toxicology Studies in Nonhuman Primates. Birth Defects Res. 86, 446-462 (2009).

12. Brennan, F. R. et al. Safety testing of monoclonal antibodies in non-human primates: Case studies highlighting their impact on human risk assessment. MAbs 10, 1-17 (2018). 
13. Emborg, M. E. Nonhuman Primate Models of Neurodegenerative Disorders. ILAR J. 58, 190-201 (2017).

14. Reichenbach, H. Longevity of mammals in captivity; from the Living Collections of the world. (2005).

15. Tacutu, R. et al. Human Ageing Genomic Resources: new and updated databases. Nucleic Acids Res. 46, 1083-1090 (2018).

16. Tacutu, R. et al. AnAge entry for Papio hamadryas. (2017). Available at: https://genomics.senescence.info/species/entry.php?species=Papio_hamadryas

17. Mattison, J. A. et al. Impact of caloric restriction on health and survival in rhesus monkeys from the NIA study. Nature 489, 318-321 (2012).

18. Ash, H. \& Smith, H. M. B. Long - Term Data on Reproductive Output and Longevity in Captive Female Common Marmosets ( Callithrix jacchus ). Am. J. Primatol. 76, 1062-1073 (2014).

19. Cox, L. A. et al. Baboons as a model to study genetics and epigenetics of human disease. ILAR J. 54, 106-121 (2013).

20. Tardif SD et al. Reproduction in captive common marmosets (Callithrix jacchus). Comp Med. 53, 364-368 (2003).

21. Huben, S. N. Von et al. Impact of Ambient Temperature on Hyperthermia Induced by ( \pm ) 3,4-Methylenedioxymethamphetamine in Rhesus Macaques. Neuropsychopharmacology. 32, 673-681 (2007).

22. Pálková, M., Sigmund, L. \& Erkert, H. Effect of ambient temperature on the circadian activity rhythm in common marmosets, Callithrix j. jacchus (primates). Chronobiol Int. 16, 149-161 (1999).

23. Yusoff Dawood, M. \& Fuchs, F. Estradiol and Progesteronein the Maternal and Fetal Circulation in the Baboon. Biol. Reprod. 22, 179-184 (1980).

24. Barry, P. A. et al. Nonhuman Primate Models of Intrauterine Cytomegalovirus Infection. ILAR 47, 49-64 (2006).

25. Kulik, L., Amici, F., Langos, D. \& Widdig, A. Sex Differences in the Development of Social Relationships in Rhesus Macaques ( Macaca mulatta ). Int J Primatol 36, 353-376 (2015).

26. Cann, J. et al. Diabetes and Obesity Research using Nonhuman Primates. (2018).

27. Hobbs, T. R. et al. Measurement of Blood Volume in Adult Rhesus Macaques 
( Macaca mulatta ). J. Am. Assoc. Lab. Anim. Sci. 54, 687-693 (2015).

28. Weiner, L. P. Definitions and Criteria for Stem Cells. Methods Mol. Biol. 438, 3-8 (2008).

29. Smith, A. Formative pluripotency: the executive phase in a developmental continuum. Development 144, 365-373 (2017).

30. Osorno, R. et al. The developmental dismantling of pluripotency is reversed by ectopic Oct4 expression. Development 139, 2288-2298 (2012).

31. Simerly, C. R. et al. Establishment and Characterization of Baboon Embryonic Stem Cell Lines An Old World Primate Model for Regeneration and Transplantation Research. Stem Cell Res 2, 178-187 (2010).

32. Nagy, A. \& Vinterstein, K. Murine Embryonic Stem Cells. Methods Enzymol. 418, 3-21 (2006).

33. Li, T. et al. Rat embryonic stem cells produce fertile offspring through tetraploid complementation. PNAS 114, 1-6 (2017).

34. Thomson, J. et al. Embryonic stem cell lines derived from human blastocysts. Science (80-. ). 282, 1145-1147 (1997).

35. Master, Z., Mcleod, M. \& Mendez, I. Benefits, risks and ethical considerations in translation of stem cell research to clinical applications in Parkinson's disease. J Med Ethics 33, 169-173 (2007).

36. Takahashi, K. \& Yamanaka, S. Induction of Pluripotent Stem Cells from Mouse Embryonic and Adult Fibroblast Cultures by Defined Factors. Cell. 126, 663676 (2006).

37. Takahashi, K. et al. Induction of Pluripotent Stem Cells from Adult Human Fibroblasts by Defined Factors. Cell. 131, 861-872 (2007).

38. Noguchi, H., Miyagi-shiohira, C. \& Nakashima, Y. Induced Tissue-Specific Stem Cells and Epigenetic Memory in Induced Pluripotent Stem Cells. Int. J. Mol. Sci. 19, 1-11 (2018).

39. Omole, A. E., Omotuyi, A. \& Fakoya, J. Ten years of progress and promise of induced pluripotent stem cells : historical origins, characteristics , mechanisms , limitations , and potential applications. PeerJ 6, 1-47 (2018).

40. Briggs, R. \& King, T. J. Transplantation of living nuclei from blastula cells into enucleated frog eggs. Zoology 38, 455-463 (1952).

41. Elitt, M. S., Barbar, L. \& Tesar, P. J. Drug screening for human genetic diseases using iPSC models. Hum. Mol. Genet. 27, 89-98 (2018). 
42. Yang, G. et al. Standards for Deriving Nonhuman Primate-Induced Pluripotent Stem Cells , Neural Stem Cells and Dopaminergic Lineage. Int. J. Mol. Sci. 19, $1-15$ (2018).

43. Aoi, T. et al. Generation of pluripotent stem cells from adult mouse liver and stomach cells. Science (80-. ). 321, 699-702 (2012).

44. Aasen, T. et al. Efficient and rapid generation of induced pluripotent stem cells from human keratinocytes. Nat Biotechnol. 26, 1276-1284 (2008).

45. Lai, M. I. et al. Advancements in reprogramming strategies for the generation of induced pluripotent stem cells. J Assist Reprod Genet 28, 291-301 (2011).

46. Tiemann, U. et al. Counteracting Activities of OCT4 and KLF4 during Reprogramming to Pluripotency. Stem Cell Reports 2, 351-365 (2014).

47. Sridharan, R. et al. Role of the murine reprogramming factors in the induction of pluripotency. Cell 136, 364-377 (2012).

48. Boyer, L. A. et al. Core Transcriptional Regulatory Circuitry in Human Embryonic Stem Cells. Cell 122, 947-956 (2005).

49. Keramari, M. et al. Sox2 Is Essential for Formation of Trophectoderm in the Preimplantation Embryo. PLoS One 5, 1-16 (2010).

50. Rand, T. A., Sutou, K., Tanabe, K., Rulifson, E. \& Yamanaka, S. MYC Releases Early Reprogrammed Human Cells from Proliferation Pause via Retinoblastoma Protein MYC Releases Early Reprogrammed Human Cells from Proliferation Pause via Retinoblastoma Protein Inhibition. Cell Rep. 23, 361-375 (2018).

51. Nakagawa, M. et al. Generation of induced pluripotent stem cells without Myc from mouse and human fibroblasts. Nat Biotechnol. 26, 101-106 (2008).

52. Okita, K. et al. A more efficient method to generate integration-free human iPS cells. Nat. Methods 8, 409-412 (2011).

53. Boyerinas, B., Park, S., Hau, A., Murmann, A. E. \& Peter, M. E. The role of let7 in cell differentiation and cancer. Endocrine-Related Cancer (2010) 17, F19F36 (2010).

54. Shyh-Chang, N. \& Daley, G. Q. Lin28: Primal Regulator of Growth and Metabolism in Stem Cells. Cell Stem Cell 12, 395-406 (2013).

55. Kim, D. et al. Generation of Human Induced Pluripotent Stem Cells by Direct Delivery of Reprogramming Proteins. Cell Stem Cell 4, 472-476 (2010).

56. Kogut, I. et al. High-efficiency RNA-based reprogramming of human primary 
fibroblasts. Nat. Commun. 9, 1-15 (2018).

57. Malik N \& Mahendra S. R. A Review of the Methods for Human iPSC Derivation. Methods Mol Biol. 997, 23-33 (2013).

58. Patel, M. \& Yang, S. Advances in Reprogramming Somatic Cells to Induced Pluripotent Stem Cells. Stem Cell Rev. 6, 367-380 (2010).

59. Debowski, K. et al. Non-viral generation of marmoset monkey iPS cells by a six-factor-in-one-vector approach. PLoS One 10, 1-21 (2015).

60. Rodriguez-polo, I. et al. Baboon induced pluripotent stem cell generation by piggyBac transposition of reprogramming factors. Primate Biol., 6, 75-86 (2019).

61. Fang, R. et al. Generation of Naive Induced Pluripotent Stem Cells from Rhesus Monkey Fibroblasts. Cell Stem Cell 15, 488-496 (2014).

62. Warren, L., Ni, Y., Wang, J. \& Guo, X. Feeder-Free Derivation of Human Induced Pluripotent Stem Cells with Messenger RNA. Sci. Rep. 2, 1-7 (2012).

63. Yoshioka, N. et al. Efficient Generation of Human iPS Cells by a Synthetic Self- Replicative RNA. Cell Stem Cell. 13, 1-21 (2014).

64. Sullivan, S. et al. Quality control guidelines for clinical-grade human induced pluripotent stem cell lines. Regen. Med. 13, 859-866 (2018).

65. Polejaeva, I. \& Mitalipov, S. Stem cell potency and the ability to contribute to chimeric organisms. Reproduction 145, R81-R88 (2013).

66. Nelakanti, R. V, Kooreman, N. G. \& Wu, J. C. Teratoma Formation: A Tool for Monitoring Pluripotency in Stem Cell Research. Curr Protoc Stem Cell Biol. 32, 1-23 (2016).

67. Tsankov, A. M. et al. An improved ScoreCard to assess the differentiation potential of human pluripotent stem cells. Nat Biotechnol. 2015 33, 1182-1192 (2015).

68. Zhang, X., Cao, H., Bai, S., Huo, W. \& Ma, Y. Differentiation and characterization of rhesus monkey atrial and ventricular cardiomyocytes from induced pluripotent stem cells. Stem Cell Res. 20, 21-29 (2017).

69. Müller, F. et al. A bioinformatic assay for pluripotency in human cells. Nat Methods. 2011 8, 315-317 (2012).

70. Bock, C. et al. Reference Maps of Human ES and iPS Cell Variation Enable High-Throughput Characterization of Pluripotent Cell Lines. Cell 144, 439-452 (2011). 
71. Wu, Y. et al. Nonhuman Primate Induced Pluripotent Stem Cells in Regenerative Medicine. Stem Cells Int. 2012, 1-7 (2012).

72. Wunderlich, S. et al. Primate iPS cells as tools for evolutionary analyses. Stem Cell Res. 12, 622-629 (2014).

73. N.Marchetto, M. C. et al. Differential L1 regulation in pluripotent stem cells of humans and apes. Nature 503, 1-8 (2014).

74. Shimozawa, N., Ono, R., Shimada, M. \& Shibata, H. Cynomolgus monkey induced pluripotent stem cells established by using exogenous genes derived from the same monkey species. Differentiation 85, 131-139 (2013).

75. Liu, H. et al. Generation of Induced Pluripotent Stem Cells from Adult Rhesus Monkey Fibroblasts. Cell Stem Cell 3, 587-590 (2008).

76. Yuehong Wu, Zhang, Y., Mishra, A., Tardif, S. D. \& Hornsby, P. J. Generation of induced pluripotent stem cells from newborn marmoset skin fibroblasts. Stem Cell Res 4, 180-188 (2011).

77. Tomioka, I. et al. Generating induced pluripotent stem cells from common marmoset ( Callithrix jacchus ) fetal liver cells using defined factors, including Lin28. Genes to Cells 15, 959-969 (2010).

78. Navara, C. S. et al. Derivation of induced pluripotent stem cells from the baboon: a nonhuman primate model for preclinical testing of stem cell therapies. Cell. Reprogram. 15, 495-502 (2013).

79. Ben-nun, I. F. et al. Induced pluripotent stem cells from highly endangered species. Nat Methods 8, 2-6 (2011).

80. Fujie, Y. et al. New Type of Sendai Virus Vector Provides Transgene-Free iPS Cells Derived from Chimpanzee Blood. PLoS One 9, 1-19 (2014).

81. Navara, C. S., Chaudhari, S. \& McCarrey, J. R. Optimization of culture conditions for the derivation and propagation of baboon (Papio anubis) induced pluripotent stem cells. PLoS One 13, 1-16 (2018).

82. Sneat, P. H. A. \& Sokal, R. R. Numerical Taxonomy 1. (1966).

83. Kumar, S., Stecher, G., Tamura, K. \& Medicine, E. MEGA7 : Molecular Evolutionary Genetics Analysis version 7 . 0 for bigger datasets. Oxford Univ. Press 33, 1870-4 (2016).

84. Tiburcy, M. et al. Defined engineered human myocardium with advanced maturation for applications in heart failure modeling and repair. Circulation 135, 1832-1847 (2017). 
85. Liu, Y.-W. et al. Human ESC-Derived Cardiomyocytes Restore Function in Infarcted Hearts of Non-Human Primates. Nat Biotechnol. 36, 597-605 (2018).

86. Chong, J. J. H. et al. Human Embryonic Stem Cell-Derived Cardiomyocytes Regenerate Non-Human Primate Hearts James. Nature 510, 273-277 (2014).

87. S. Lee, A., Tang, C., Rao, M. S., Weissman, I. L. \& Wu, J. C. Tumorigenicity as a Clinical Hurdle for Pluripotent Stem Cell Therapies. Nat Med. 19, 9981004 (2014).

88. Hong, S. G., Dunbar, C. E. \& Winkler, T. Assessing the Risks of Genotoxicity in the Therapeutic Development of Induced Pluripotent Stem Cells. Mol. Ther. 21, 272-281 (2013).

89. Rosenzweig, E. S. et al. Restorative Effects of Human Neural Stem Cell Grafts to the Primate Spinal Cord. Nat Med. 24, 484-490 (2018).

90. Nemati, S. et al. Transplantation of Adult Monkey Neural Stem Cells into A Contusion Spinal Cord Injury Model in Rhesus Macaque Monkeys. CELL J. 16, 117-130 (2014).

91. Shirai, H. et al. Transplantation of human embryonic stem cell-derived retinal tissue in two primate models of retinal degeneration. PNAS 113, 81-90 (2016).

92. Shiba, Y. et al. Allogeneic transplantation of iPS cell-derived cardiomyocytes regenerates primate hearts. Nature 538, 388-391 (2016).

93. Liu, Y. et al. Human embryonic stem cell - derived cardiomyocytes restore function in infarcted hearts of non-human primates. Nat. Publ. Gr. 36, 597-605 (2018).

94. Shi, Q., Schatten, G., Hodara, V., Simerly, C. \& Vandeberg, J. L. Endothelial reconstitution by $\mathrm{CD} 34$ + progenitors derived from baboon embryonic stem cells. J. Cell. Mol. Med. Vol 17, 242-251 (2013).

95. Kavyasudha, C. et al. Clinical Applications of Induced Pluripotent Stem Cells - Stato Attuale. Adv Exp Med Biol 1, 127-149 (2018).

96. Allison, T. F. \& Lowry, W. E. The reprogramming method matters. Nat. Biomed. Eng. 1, 779-781 (2017).

97. Yoshimatsu, S. et al. Robust and efficient knock-in in embryonic stem cells and early- stage embryos of the common marmoset using the CRISPR-Cas9 system. Sci. Rep. 9, 1-12 (2019).

98. Yao, X. et al. Generation of knock-in cynomolgus monkey via CRISPR / Cas9 editing. Cell Res. 28, 379-382 (2018). 
99. Boroviak, T. et al. Single cell transcriptome analysis of human, marmoset and mouse embryos reveals common and divergent features of preimplantation development. Development 145, 1-18 (2018).

100. Guo, W., Bharmal, S. J., Esbona, K. \& Greaser, M. L. Titin diversity-alternative splicing gone wild. J. Biomed. Biotechnol. 2010, 1-8 (2010).

101. Perleberg, C., Kind, A. \& Schnieke, A. Genetically engineered pigs as models for human disease. Co. Biol. 11, 1-12 (2018).

102. Cardoso-Moreira, M. et al. Gene expression across mammalian organ development. Nature 571, 505-509 (2019).

103. Wolf, N. S. \& Austad, S. The Comparative Biology of Aging. Introduction: Lifespans and Pathologies Present at Death in Laboratory Animals. (Springer, Dordrecht, 2009).

104. Chong, J. J. H. \& Murry, C. E. Cardiac Regeneration Using Pluripotent Stem Cells - Progression to Large Animal Models. Stem Cell Res. 13, 654-665 (2014).

105. Bert, A. A. et al. Transesophageal echocardiography in healthy young adult male baboons ( Papio hamadryas anubis ): Normal cardiac anatomy and function in subhuman primates compared to humans. Prog. Pediatr. Cardiol. 35, 109-120 (2013).

106. Milani-Nejad, N. \& Janssen, P. M. L. Small and Large Animal Models in Cardiac Contraction Research: Advantages and Disadvantages. Pharmacol Ther 141, 235-249 (2014).

107. Oberhoffer, R. M., Ho, S. Y. \& Anderson, R. H. Coronary Artery Diameters in the Heart With Complete Transposition Of the Great Vessels. JACC 15, 14331437 (1990).

108. Gest, T. R. \& Siegel, M. I. The Relationship Between Organ Weights and Body Weights , Facial Dimensions , and Dental Dimensions in a Population of Olive Baboons ( Papio cynocephalus anubis ). Am. J. Phys. Anthropol. 61, 189-196 (1983).

109. Kuo, A. H. et al. Cardiac remodelling in a baboon model of intrauterine growth restriction mimics accelerated ageing. J Physiol 595, 1093-1110 (2017).

110. Brener, H. et al. Coronary Artery Plaque Rapidly Induced by Local Electromagnetic Stimulation in the Baboon. Eur Surg Res 21, 123-128 (1989).

111. Larson, S. G. Scaling of Organ Weights in Macaca arctoides. AM. J. PHYS. 
Anthr. 49, 95-102 (1978).

112. Sasseville, V. G., Hotchkiss, C. E., Levesque, P. C. \& Mankowski, J. L. Hematopoietic, Cardiovascular, Lymphoid and Mononuclear Phagocyte Systems of Nonhuman Primates. in Nonhuman Primates in Biomedical Research 357-384 (Elsevier Inc., 2012).

113. Teofilovski-Parapid, G. \& Kredovit, G. Coronary artery distribution in Macaca fascicularis ( Cynomolgus ). Lab. Anim. 32, 200-205 (1998).

114. Senos, R. et al. Gross morphometry of the heart of the Common marmoset. Folia Morphol 73, 37-41 (2014).

115. Robert Marini et al. The Common Marmoset in Captivity and Biomedical Research. (Academic Press, 2018).

116. Ansel, T. V, Nour, A. K. \& Benavente-perez, A. The Effect of Anesthesia on Blood Pressure Measured Noninvasively by Using the Tail-Cuff Method in Marmosets ( Callithrix jacchus ). J. Am. Assoc. Lab. Anim. Sci. 55, 594-600 (2016).

117. Thtiroff, J. W., Hort, W. \& Lichti, H. Diameter of coronary arteries in 36 species of mammalian from mouse to giraffe. Basic Res. Cardiol. 206, 199-206 (1984).

118. Kassab, G. S., Rider, C. A., Tang, N. J. \& Fung, Y.-C. B. Morphometry of pig coronary arterial trees. Am J Physiol. 1, 350-365 (1993).

119. Gómez, F. A., Cortés, L. S. \& Ballesteros, L. E. Morphological characterisation of the coronary arteries in African sheep ( Ovis orientalis ). Differential analysis with those of humans and other animal species. Folia Morphol. 78, 63-70 (2019).

120. Gerova, M., Barta, E. \& Gero, J. A. N. Sympathetic Control of Major Coronary Artery Diameter in the Dog. Circ. Res. 44, 459-67 (1964).

121. Sharma, R. \& Singh, R. B. MRI of coronary artery atherosclerosis in rabbits : Histopathology-MRI correlation and atheroma characterization. Thromb. J. 2004, 12, 1-12 (2004).

122. Sanjana, N. E. et al. A Transcription Activator-Like Effector (TALE) Toolbox for Genome Engineering. Nat Protoc 7, 171-192 (2013).

123. Jehuda, R. Ben, Shemer, Y. \& Binah, O. Genome Editing in Induced Pluripotent Stem Cells using CRISPR / Cas9. Stem Cell Rev Rep 3, 323-336 (2018).

124. Barrangou, R. et al. CRISPR provides acquired resistance against viruses in prokaryotes. Science (80-. ). 315, 1709-1713 (2007). 
125. Petkov, S. et al. Immortalization of common marmoset monkey fibroblasts by piggyBac transposition of hTERT. PLoS One 13, 1-13 (2018).

126. Hill, J. T. et al. Poly Peak Parser: Method and software for identification of unknown indels using Sanger Sequencing of PCR products. Dev Dyn. 243, 1632-1636 (2015).

127. Haake, K., Ackermann, M. \& Lachmann, N. Concise Review: Towards the Clinical Translation of Induced Pluripotent Stem Cell-Derived Blood Cells Ready for Take-Off. Stem Cells Transl. Med. 8, 332-339 (2019).

128. Nakai, R., Ohnuki, M., Kuroki, K., Ito, H. \& Hirai, H. Derivation of induced pluripotent stem cells in Japanese macaque ( Macaca fuscata ). Sci. Rep. 8, 1-9 (2018).

129. Yada, R. C. et al. Rhesus Macaque iPSC Generation and Maintenance. Curr Protoc Stem Cell Biol. 41, 1-17 (2018).

130. Mishra, A. et al. Induced pluripotent stem cells from nonhuman primates. Methods Mol Biol. 1357, 183-193 (2017).

131. Rodriguez-polo, I., Nielsen, M., Debowski, K. \& Behr, R. The ubiquitin ligase c-CBL is expressed in undifferentiated marmoset monkey pluripotent stem cells but is not a general stem cell marker. Primate Biol 4, 231-240 (2017).

132. Baghbaderani, B. A. et al. cGMP-Manufactured Human Induced Pluripotent Stem Cells Are Available for Pre-clinical and Clinical Applications. Stem Cell Reports 5, 647-659 (2015).

133. Shafa, M., Yang, F., Fellner, T., Rao, M. S. \& Baghbaderani, B. A. humaninduced Pluripotent stem cells Manufactured Using a current good Manufacturing Practice-compliant Process Differentiate into clinically relevant cells From Three germ layers. Front. Med. 5, 1-12 (2018).

134. Peterson, S. E. \& Loring, J. F. Genomic Instability in Pluripotent Stem Cells : Implications for Clinical Applications. J. Biol. Chem. 289, 4578-4584 (2014).

135. Dunn, K. K. \& Palecek, S. P. Engineering Scalable Manufacturing of HighQuality Stem Cell-Derived Cardiomyocytes for Cardiac Tissue Repair. Front. Med. | 5, 1-18 (2018).

136. Tohyama, S. et al. Distinct Metabolic Flow Enables Large-Scale Purification of Mouse and Human Pluripotent Stem Cell-Derived Cardiomyocytes. Stem Cell 12, 127-137 (2013).

137. Lin, Y. et al. Efficient differentiation of cardiomyocytes and generation of 
calcium-sensor reporter lines from nonhuman primate iPSCs. Sci. Rep. 8, 1-16 (2018).

138. Hattori, F. et al. Nongenetic method for purifying stem cell - derived cardiomyocytes. Nat Methods 7, 61-67 (2010).

139. Godier-Furnémont, A. et al. Physiologic force-frequency in engineered heart muscle by electromechanical stimulation. Biomaterials. 60, 82-91 (2015).

140. Sala, L., Ward-van Oostwaard, D., Tertoolen, L. G. J., Mummery, C. L. \& Bellin, M. Electrophysiological Analysis of human Pluripotent Stem Cellderived Cardiomyocytes ( hPSC-CMs ) Using Multi-electrode Arrays ( MEAs ). J. Vis. Exp. 123, 1-15 (2017).

141. Cox, L. A. et al. Nonhuman Primates and Translational Research Cardiovascular Disease. ILAR J. 58, 235-250 (2017).

142. Yang, S. et al. Towards a transgenic model of Huntington's disease in a nonhuman primate. Nature 453, 921-924 (2009).

143. Sasaki, E. et al. Generation of transgenic non-human primates with germline transmission. Nature 459, 523-527 (2009).

144. Shi, L. et al. Transgenic rhesus monkeys carrying the human MCPH1 gene copies show human-like neoteny of brain development. Natl. Sci. Rev. 6, 480493 (2019).

145. Yada, R. C. et al. CRISPR/Cas9-based safe-harbor gene editing in rhesus iPSCs. Curr Protoc Stem Cell Biol 43, 1-21 (2018).

146. Chen, Y. \& Pruett-miller, S. M. Improving single-cell cloning work flow for gene editing in human pluripotent stem cells. Stem Cell Res. 31, 186-192 (2018).

147. Mestroni, L., Brun, F., Spezzacatene, A., Sinagra, G. \& RG Taylor, M. Genetic causes of dilated cardiomyopathy. Prog Pediatr Cardiol. 37, 13-18 (2015).

148. Hershberger, R. E., Morales, A. \& Siegfried, J. D. Clinical and genetic issues in dilated cardiomyopathy: A review for genetics professionals. Genet Med 12, 655-667 (2010).

149. Zhang, Y., Long, C., Bassel-duby, R. \& Olson, E. N. Myoediting: Toward prevention of muscular dystrophy by therapeutic genome editing. Physiol Rev 98, 1205-1240 (2018).

150. Wojtal, D. et al. Spell Checking Nature : Versatility of CRISPR / Cas9 for Developing Treatments for Inherited Disorders. Am. J. Hum. Genet. 98, 1-12 
(2016).

151. Kyrychenko, V. et al. Functional correction of dystrophin actin binding domain mutations by genome editing Find the latest version : Functional correction of dystrophin actin binding domain mutations by genome editing. JCI Insight $\mathbf{2}$, $1-16$ (2017).

152. Li, H. L. et al. Precise Correction of the Dystrophin Gene in Duchenne Muscular Dystrophy Patient Induced Pluripotent Stem Cells by TALEN and CRISPR-Cas9. Stem Cell Reports 4, 143-154 (2015).

153. Long, C. et al. Correction of diverse muscular dystrophy mutations in human engineered heart muscle by single-site genome editing. Sci. Adv. 4, 1-12 (2018).

154. Zhang, Y. et al. CRISPR-Cpf1 correction of muscular dystrophy mutations in human cardiomyocytes and mice. Sci. Adv. 3, 1-10 (2017).

155. Bengtsson, N. E. et al. Muscle-specific CRISPR/Cas9 dystrophin gene editing ameliorates pathophysiology in a mouse model for Duchenne muscular dystrophy. Nat. Commun. 8, 1-9 (2017). 


\section{Acknowledgments}

First, I would like to thank my thesis committee for the support during my Ph.D. Additionally, I would like to thank all different individual researchers and research groups that I had the luck to collaborate with.

When a student chooses a Ph.D. project the decision is based mainly on the research interest, how the student will fit into the group is question of luck. In my case, I was extremely lucky to be able to do this project in an amazing and supportive working environment surrounded by great researchers and amazing persons. I had the luck to count on very talented postdocs, Michael and Stoyan, from whom I have learned a lot. Especially I want to thank the best TAs in the world Angelina, Nicole, Carmen, Ulrike and Anna which support and assistance during all these years have improved not only my work but also my stay in the lab. Also, I want to thank Charis and the rest of the components of Degenerative disease research platform for the nice moments expend together during these years. Finally, I would like to thank my boss. Thank you Rüdiger for giving me this opportunity, for the support, for having the patience of listening to all kind of crazy ideas seasoned with Spanish accent and for sharing with me this journey.

During my Ph.D. also I have the luck to supervise many talented students. With them, I have discovered the pleasure in teaching, show, and share science with persons with the same passion for it that I have.

Finally, I will like to thank my family and friends.

THANK YOU! 


\section{Curriculum Vitae}

Ignacio Rodríguez Polo

Date of Birth: 13th July 1990

Place of Birth: Madrid, Spain

E-mail: $\underline{\text { IRodriguezPolo@dpz.eu }}$

\section{Education and training}

\section{1/12/2015- Expected graduation (October 2019)}

Ph.D. degree - Thesis title: Non-human primate iPS cells for cell replacement therapies and human cardiovascular disease modeling

Platform Degenerative Diseases, German Primate Center (Deutsches Primatenzentrum, DPZ), Göttingen, Germany

\section{$30 / 08 / 2014-30 / 09 / 2015$}

Research assistant. Research project title: Dissecting miRNA gene repression on single-cell level with an advanced fluorescent reporter system Medical RNA Biology group. German Primate Center (Deutsches Primatenzentrum, DPZ), Göttingen, Germany

\section{8/02/2014-30/08/2014}

Master thesis like (diploma thesis). Master thesis title: Functional Characterization of Zinc Cluster transcription factors in relation with resistance of the human pathogen Aspergillus fumigatus

Dept. Microbiology and Genetics, Georg-August-Universität Göttingen, Germany

\section{1/09/2012- 30/06/2013}

Research assistant (Bachelor thesis like). Research project title: Network's topology describes the nematode communities assemblages in post reclaimed landfills Department of Ecology, Aristotle University of Salonica. Thessaloniki, Greece 


\section{1/09/2008-30/09/2014}

Licenciatura in biology (Diploma degree).

Complutense University of Madrid, Spain

\section{List of Publications}

Rodriguez-polo, I., Stauske, M., Becker, A., Bartels, I., Dressel, R. and Behr, R.: Baboon induced pluripotent stem cell generation by piggyBac transposition of reprogramming factors, Primate Biol., 6(2), 75-86, doi:https://doi.org/10.5194/pb-675-2019, 2019.

Wahab, F., Khan, I. U., Rodriguez-Polo, I., Zubair, H., Drummer, C., Shahab, M. and Behr, R.: Irisin in the primate hypothalamus and its effect on GnRH in vitro, $J$. Endocrinol., 241(3), 175-187, doi:https://doi.org/10.1530/JOE-18-0574, 2019.

Rodriguez-Polo, I., Nielsen, M., Debowski, K. and Behr, R.: The ubiquitin ligase c$\mathrm{CBL}$ is expressed in undifferentiated marmoset monkey pluripotent stem cells but is not a general stem cell marker, Primate Biol., 4, 231-240, doi: https://doi.org/10.5194/pb-4-231-2017, 2017.

Lemus-diaz, N., Böker, K. O., Rodriguez-polo, I., Mitter, M., Preis, J., Arlt, M. and Gruber, J.: Dissecting miRNA gene repression on single cell level with an advanced fluorescent reporter system, Sci. Rep., 7(45197), 1-15, doi:10.1038/srep45197, 2017.

\section{Teaching experience}

09/2019 Supervision of Master Thesis. Yuliia Tereshchenko. GGNB Molecular Biology - International Max Planck Research School (MSc/Ph.D.).

03/2019 Supervision of Master Lab Rotation. Iga Grządzielewska. GGNB Molecular Biology - International Max Planck Research School (MSc/Ph.D.).

01/2019 Supervision of Master Lab Rotation. Yuliia Tereshchenko. GGNB Molecular Biology - International Max Planck Research School (MSc/Ph.D.). 
10/2018 Supervision of Master Lab Rotation. Michelle Chan. Molecular Medicine- Universitätsmedizin Göttingen (UMG) (M.Sc.).

04/2018 Supervision of Master Thesis. Daniel Urrutia Cabrera. Developmental, Neural, and Behavioral Biology (MSc.).

02/2018 Supervision of Master Thesis. Debbra Yasemin Knorr. Developmental, Neural, and Behavioral Biology (MSc.).

02/2018 Supervision of Master Lab Rotation. Daniel Urrutia Cabrera. Developmental, Neural, and Behavioral Biology (MSc.).

02/2016 Supervision of B.Sc. Lab Rotation. Alexander Becker. Biologie (B.Sc.) 2016/2017/2018/2019 Tutorial focus on stem cells. GGNB Molecular Biology - International Max Planck Research School (MSc/Ph.D.).

\section{$\underline{\text { Additional activities during the doctorate }}$}

2017-2018 Leibniz Ph.D., student representative. German Primate Center (Deutsches Primatenzentrum, DPZ), Göttingen

2016-2017 Organizing Committee. Göttingen Biotech Symposium 2017. German Primate Center (Deutsches Primatenzentrum, DPZ), Göttingen 
Curriculum Vitae 